NBSIR 80-2164 $\uparrow$

\title{
Technical Activities 1980 Center for Analytical Chemistry
}

C. W. Reimann, R. A. Velapoldi, L. B. Hagan, and J. K. Taylor, Editors

National Measurement Laboratory

National Bureau of Standards

U.S. Department of Commerce

Washington, DC 20234

October 1980

Final

Issued December 1980

Prepared for

National Bureau of Standards

Department of Commerce

Washington, D.C. 20234 



\section{TECHNICAL ACTIVITIES 1980 \\ CENTER FOR ANALYTICAL CHEMISTRY}

C. W. Reimann, R. A. Velapoldi, L. B. Hagan, and J. K. Taylor, Editors

National Measurement Laboratory

National Bureau of Standards

U.S. Department of Commerce

Washington, DC 20234

October 1980

Final

Issued December 1980

Prepared for

National Bureau of Standards

Department of Commerce

Washington, D.C. 202340

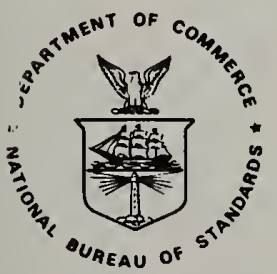

U.S. DEPARTMENT OF COMMERCE, Philip M. Klutznick, Secretary Jordan J. Baruch, Assistant Secretary for Productivity, Technology, and Innovation NATIONAL BUREAU OF STANDARDS, Ernest Ambler, Director 

I. CENTER FOR ANALYTICAL CHEMISTRY

A. Center Overview .................... . . 1

B. Voluntary Standardization and Qual ity Assurance

Coordination. . . . . . . . . . . . . . 4

C. National Environmental Specimen Bank. . . . . . . . 8

D. Service Analysis. . . . . . . . . . . . . 21

E. New Competence Building Program - FY '81. . . . . . . 23

F. Instrument Development Group. . . . . . . . . . . 26

1. Group Overview ............... 26

2. Selected Technical Accomplishments . . . . . . . 28

a. Studies of the Charge-Collection Mechanism in Laser Enhanced Ionization Spectrometry. . . . 28

b. Atomic Resonance-Line Lasers for Atomic Spectrometry. ............. . 33

c. Reduction of Ionization Interferences in Laser Enhanced Ionization Spectrometry....... . 36

d. Detection Limits of Some Trace Molecules of the Atmosphere by IR Diode Laser Absorption . . . . 38

e. Trace Organic Analys is by Multiphoton Ioniza-

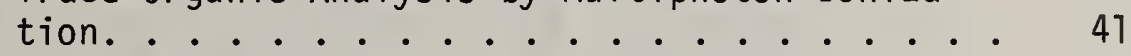

f. Instrumentation Project.......... . . 45

3. Outputs and Interactions ............ 46

\section{I. INORGANIC ANALYTICAL RESEARCH DIVISION}

A. Division Overview ............... . . . . 49

B. Selected Technical Accomplishments. . . . . . . . . . 59

1. D.C. PTasma Emission Spectrometry. . . . . . . . . 59

2. The Halogen Content of Lubricating 0ils. . . . . . 61

3. Measurement of the Co-60 to Co-59 Ratio in Microparticles.................. 62

4. Cryogenic Homogenization of Tissue . . . . . . . 64

5. Rapid Analys is of Toxic Metals in Biological Tissue and Fluids by Atomic Absorption Spectrometry . . .

6. Comparative Measurements of Zinc-70 Enrichment in Human Plasma Using Neutron Activation Analys is and Thermal Ionization Mass Spectrometry.......

7. Trace and Toxic Elemental Study of the Chesapeake Bay.................... . . 69

8. Characterization of Potentialiy Hazardous Wastes Development of Rotary Extractor and Quality Assurance Standards.............. 72

9. Development of an Improved Gravimetric Method for the Determination of Aluminum in Bauxite . . . . 75

10. Fission Track Monitor. . . . . . . . . . . . 77

11. Interferences in the Inductively Coupled Plasma (ICP) Technique. .............. 77

12. Atomic Weight of Silver............ . . 79 
13. High Accuracy Mass Spectrometric Isotopic Analys is of Uranium .............. 81

14. Half-Life of $24 i \mathrm{pu} \ldots 82$

15. High Precision Pulse-Counting. . . . . . 83

16. High Accuracy Method for Determining Sulfur in

Iron Base Alloys . . . . . . . . . . 84

17. Standard Reference Materials ......... 86

C. Outputs and Interactions. ............ 89

\section{ORGANIC ANALYTICAL RESEARCH DIVISION}

A. Division Overview .............. 99

B. Selected Technical Accomplishments. ........ 110

1. Isotope Dilution/Mass Spectrometry Definitive Method Developed for Creatinine in Serum. . . . . . 110

2. Human Serum Reference Material (SRM 909) ...... 111

3. Fluoroimmunoassay Procedure Developed for Dinitrophenols in Water. . . . . . . . 112

4. Development of Methods for Determination of the Aromatic Carbon Content of Recycled 0ils by HighField ${ }^{13} \mathrm{C}$ NMR Spectroscopy

5. Research on Two-Dimensional Proton NMR Spectroscopy and Its Application to SRM D-Glucose .......

6. Analys is of Sodium Pyruvate and Its Impurities by Proton NMR Spectroscopy at $400 \mathrm{MHz}$. . . . . . 121

7. Analysis of Boat Conformations of $\alpha-\dot{D}$-Aitropyranoside Derivatives by Interpretation of High̄ly Coupled

8. Laser Polarimetry and Its Appi ication to Recertification of Sucrose SRM (SRM 17c).

9. Certification of Generator Columns for Polynuclear Aromatic Hydrocarbons in Water (SRM 1644).

10. First Natural Matrix Trace Organic SRM Issued for Shale 0i1 (SRM 1580) ............. 134

11. Synthesis, Isotopic and Purity Analyses, and Preparation of Solutions of Stable Isotope Labeled Marker Compounds for Priority Pollutant Analysis. . . . . 136

12. Evaluation of a Spectroelectrochemical System Using o-Tolidine ............ 138

13. Development of a System for Computer-Controlled Rapid El ectrochemical Measurements . . . . . 140

14. Development of a High-Sensitivity Duai-El ectrode Detector for Liquid Chromatography . . . . . . . 141

15. Estimation of Thermodynamic and Kinetic Data from Spectroelectrochemical Studies Using Digital Simulations.

16. Method Development for Determination of Ambient Temperature Vapor Pressures of Polynuclear Aromatic Hydrocarbons

17. Combined LC/MS Techniques for Direct Quantitative
Analysis of Individual Organic Compounds in Complex

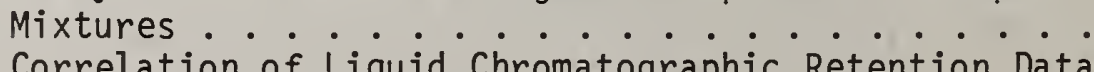

18. Correlation of Liquid Chromatographic Retention Data with Molecular Shapes for Polycyclic Aromatic Hydrocarbons 
19. Application of Multi-Dimensional Chromatography to

Trace Organic Compound Quantitation ....... 150

20. Development of a Procedure for the Analys is of

Polychlorinated Biphenyls in Recycled 0i1s. . . . . 152

21. Development of a Fourier Transform Infrared

Spectrometer-Gas Chromatograph Interface and Its

Application to Pollutant Identification ...... 153

c. Outputs and Interactions ............ 154

IV. GAS AND PARTICULATE SCIENCE DIVISION

A. Division Overview. ............ 165

B. Selected Technical Accomplishments ......... 174

1. Intercomparison of $\mathrm{O}_{3}$ and $\mathrm{NO}_{2}$ Measurement Standards

by Gas Phase Titration. . . . . . . . . . . 174

2. Development of Atmospheric Nitrous Oxide and Halo-

carbon Standards. . . . . . . . . . . 176

3. The Determination of Infrared Line Intensities for

Selected Molecules Using Diode Laser and Fourier

Transform Infrared Spectroscopy . . . . . . . . 179

4. The Measurement of Temperature Dependence of Ozone

Absorption Cross-Sections in the Ultraviolet. . . . . 180

5. Standards for Measurement of Atmospheric Carbon

Dioxide . . . . . . . . . . . . . 182

6. Industrial Preparation of Traceable Gas Certified

Reference Materials . . . . . . . . . . . 183

7. Standard for Measurement of Benzene and Perchlor-

ethylene in Workplace Atmospheres . ........ 184

8. Motor Vehicle Manufacturers Association Program for

Heavy Duty Vehicle Emissions Standards. . . . . . . 185

9. Evaluation of the Coherent Anti-Stokes Raman

Spectroscopy (CARS) Capillary Waveguide Detection

System for High Performance Liquid Chromatography . .

10. Measurement of Ambient Sulfur Aerosols by a New Fast

Response Flame Photometric Instrument . . . . . .

11. Monte Carlo Electron Trajectory Calculation of Beam

Spreading Effects in Thin Foils in the Analytical

El ectron Microscope ..............

12. General Expression Developed for the X-ray

Bremsstrahlung Intensity Data . . . . . . . . 192

13. Development of a Sequential Simplex Procedure for

Resolving Spectral Interference in Energy Dispersive

X-ray Spectrometry. . . . . . . . . . . .

14. Development of a New Procedure for Calibration of a

Crystal Spectrometer for Relative Line Intensity

Measurements. . . . . . . . . . . 195

15. Successfur Raman Microprobe Analys is of Opaque

Particles ............... 197

16. Development and Characterization of a New Raman

Microprobe with Multichannel Optical Detector . . . . 200

17. Ion Microprobe and Laser Microprobe Mass Analysis of

Inorganic Particulates. . . . . . . . . 
18. Evaluation of Counter Error in the Development of Asbestos Standards for the Electron Microscope . . . 206

19. The Development of Thin Glass Films as Potential Standards for X-ray Spectrometric Calibration. . . . . 207

20. Low Velocity Wind Tunnel Facility for the Evaluation of Personnel Dosimeters. . . . . . . . . . 211

21. Enhanced Detection of Molecules Near a Surface by Raman Spectroscopy Excited by Evanescent Waves . . . . 212

22. Statistical Evaluations of the International Radiocarbon Cross Calibration Exercise, and of the Natural Radiocarbon Fluctuations ............ 214

23. Advances in Small Sample Radiocarbon Measurement Techniques; Application to the Assay of Individual Atmospheric Chemical Species.......... 220

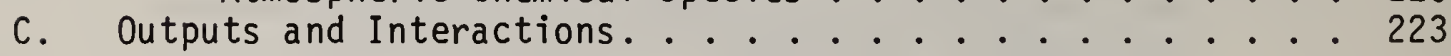




\section{Center for Analytical Chemistry}

Curt W. Reimann, Director

Rance A. Velapoldi, Deputy Director

\section{A. Center Overview}

The Center for Analytical Chemistry develops and maintains the scientific competences and the experimental facilities necessary to provide the Nation with the basis for uniform measurements through development of measurement methodology and provision of measurement services in the field of analytical chemistry. Analytical measurements provide a foundation for all aspects of scientific and technological research and development concerning materials and their practical application. Furthermore, results of analytical measurements are increasingly used as the basis for regulatory, medical, and industrial decisions. It is essential that these measurements be reliable and the Center serves as the national reference laboratory for assuring this reliability.

The principal means by which the Center serves as a national reference laboratory is through the development and issuance of standard reference materials (SRM's). The ubiquity of and demand for analytical measurements in modern society is reflected in the diversity of SRM's produced in the Center. These standards are used in the metal and chemical industries and in clinical and environmental laboratories. Each standard is supported by research on homogeneity and stability and is certified as to chemical composition using the most reliable analytical methods available.

Analytical chemistry is increasingly being called upon to provide key data for highly complex sample types - hazardous chemical and nuclear wastes, body fluids, $\mathrm{plant}$ and animal tissue, foods, and high-technology materials. Often analytical values as a function of location within a sample are needed. As the demands upon analytical chemistry become more sophisticated, the problem of standardization becomes more challenging both in terms of the number and the complexity of the proposed standard materials. As it is not reasonable to contemplate the development of complex standards to deal with every measurement problem, the Center's program must be multifaceted, seeking a variety of avenues to impact the quality of analytical measurements made in the U.S. In addition to the issuance of standards, the Center exerts influence through close contacts with various communities by means of workshops, symposia, and participation in standards committees. Through such contacts, priorities are established for the production of standards and mechanisms are set in place for their effective use. 
The program of the Center strives to achieve a balance among the provision of currently needed standards and research efforts on new types of standards and measurement methods. The work of the Center is performed in three divisions, each focusing upon specific sample types - inorganics, organics, and gases and particulates. Within each division there are several groups representing separate analytical chemical disciplines, e.g., mass spectrometry and electrochemistry. One group, the Instrumentation Development Group, is attached to the Center office. Each division is responsible for a program in basic and applied research and maintains contacts with appropriate communities.

In the following, the major recent activities and technical directions of the divisions and groups mentioned above are given. Much work in progress is omitted but will be included in subsequent Annual Reports.

1. Outputs and Interactions

a. Publications

Brewer, Leo and Hagan, Lucy, "The Oscillator Strength of the $C_{2}$ Swan Bands", High Temperature Science, 11, 233-264 (1979).

Reimann, Curt W. and Hagan, Lucy, "Technical Activities 1979, Center for Analytical Chemistry", NBSIR 80-1995 (March 1980).

b. Committee Assignments

Lucy Hagan

Member, NBS Synfuels Task Force

Member, CAC Committee on Computer Requirements

c. Center Colloquium Series

October 1, $1979 \quad$ - Graeme Batley, Australian Atomic Energy Commission, Lucas Heights, Australia, "Electrodeposition Techniques for the Study of Heavy Metals in Natural Waters".

October $9 \quad-\quad$ Steven C. Wofsy, Harvard University, "Some Recent Problems of Atmospheric Chemistry".

October 30 - Morteza Janghorbani, Massachusetts Institute of Technology, "Stable Isotope Tracers in Human Mineral Nutrition".

November 14 - Charles E. Kolb, Aerodyne Research, Bedford, MA, "Laboratory Applications of Tunable Diode Lasers". 
November 15,197

November 30

December 3

December 12

January 16,1980

February 13

March 14

March 21

Apri1 9

May 14

June 13

June 18
Charles A. Evans, Charles Evans Associates, San Mateo, CA, "Recent Advances in Materials Analysis by Secondary Ion Mass Spectroscopy".

- Andrew Tam, IBM, San Jose, CA, "Laser Optoacoustic Spectroscopy".

- Bruce Kowalski, University of Washington, "Chemometrics in Analytical Chemistry".

- John I. Peterson, NIH, Bethesda, MD, "The Fiber Optic pH Probe".

- William Dark, Waters Associates, Milford, MA, "An Overview of HPLC for Clinical Analysis".

- Geraldine M. Huitink, Indiana University of South Bend, South Bend, IN, "The Role of the Fluorophore in Metallofluorescent Indicators".

- Richard Van Duyne, Northwestern University, Evanston, IL, "Recent Developments in Enhanced Raman Spectroscopy".

- David M. Coleman, Wayne State University, Detroit, MI, "Studies in New Analytical Segregated Electrical Discharge Systems".

- Jack W. Frazer, Lawrence Livermore Laboratory, Berkeley, CA, "Characterization and Control of Complex Chemical Systems".

- Velmer A. Fassel, Iowa State University, Ames, IA, "Inductively Coupled Plasma-Atomic Emission Spectroscopy: Past, Present, and Future".

- John C. Wright, University of Wisconsin, Madison WI, "Application of Selective Laser Excitation Studies to Solid State Chemistry and U1tra-Trace Analysis".

- John T. Stock, University of Connecticut, "Historic Instruments: The Scientist's Heritage". 


\section{B. Voluntary Standardization and Quality Assurance Coordination}

\section{Voluntary Standardization}

Participation in the voluntary standardization process is an important activity of members of the staff of the Center. Committee assignments, listed under the respective divisional summaries, indicate that 24 members, i.e., 20 percent of the staff, hold memberships on 97 national and international organizations. The extent of participation ranges from general review of documents to holding of major offices. During the past year, positions of leadership were held on 12 groups or committees, and Center staff actively authored a number of standards and participated in four collaborative tests.

Participation in standardization activities has hitherto been on a rather informal basis. While some has resulted from direct invitations for NBS to participate, most has stemmed from the personal interests of the staff, which NBS has always had the policy to encourage. As a result, there are a number of committees and/or task groups in which there is multiple representation and such redundancy is not without benefit. On the other hand, there are doubtless other groups which may be in need of the Center's expertise, where it is presently lacking. Recent Developments in standardization policy and outlook emphasize the need for some coordination of the Center's standardization activities.

Under provisions of Circular A-119 issued by the Office of Management and Budget in January 1980, entitled, "Federal Participation in the Development and Use of Voluntary Standards", government organizations are encouraged to participate in development of, and to make more use of voluntary standards as they relate to their areas of interest. The Circular limits participation to those organizations which have the broadest representation of the areas of interest and which fully utilize due-process practices in standards development.

CAC expertise can well serve these objectives. Through a wider base of service, the Center could provide increased services to existing constituents and perhaps broaden its base to constituents not presently reached. A careful search for areas of need may identify new opportunities. Because of the amount of present activity, it might be necessary to limit some multiple representations in order to extend service to others.

A further need for coordination results from the growing concern about "high impact" standards. Examples of such standards are those with considerable economic impact, or that would involve policy decisions in their development. Furthermore, a number of standards committees are becoming classified, i.e., membership is classified to assure a balance of interests, hence, coordination of position across laboratory or agency lines may need to be established. As a minimum, more record keeping will be required not only in the sensitive areas, but in others as well, since there is no certain way to identify, before hand, when a high-impact issue might develop within any committee. 


\section{Quality Assurance}

The growing interest in quality assurance of analytical data is making increasing demands on the Center's programs while influencing its' internal activities as well. The Center has always had concern for the quality of its data and its quality assurance program might be described as one of informal redundancy. Key measurements, such as standard reference material (SRM) certifications, for example, are made using at least two independent methods and often with the number of repetitions far exceeding the minimum requirements. However, interagency agreements involving data production are or will be requiring formal Quality Assurance programs to be used. For this and other reasons, a critical survey will be made of the Center's existing Quality Assurance programs to see if and/or where they need strengthening. The results should be internally beneficial, as well as helpful to others in designing their own Quality Assurance programs.

Another area related to quality assurance is the proper utilization of SRM's. While the end-purpose of these is for the improvement of measurement accuracy, no formal instructions for their use, in this respect, are ordinarily available from NBS. As SRM's are being obtained by a growing community of users, the need for more formal applications information is emerging. Such guidelines or instructions would extend their usefulness, alleviate possible misuse, and conceivably extend the supply in some cases.

A protocol for industrial production of certified reference materials, traceable to NBS SRM's is under development by the Gas and Particulate Science Division, in collaboration with the Environmental Protection Agency. This protocol describes in detail how the SRM should be used to assure the quality of the measurement of the certified reference material. While protocol development may be useful in only a limited number of cases, there are others where procedures could be recommended to maximize the usefulness of the SRM. Center scrutiny will be encouraged to identify such cases, especially while certifying a specific SRM, so that applications for information can be developed concurrently.

John K. Taylor

3. Outputs and Interactions

a. Publications

Taylor, J. K., "The Importance of Intercalibration in Marine Analysis", Tha1. Jugoslay., 14 (1/2) 221-229 (1978) (Delayed publication).

Taylor, J. K., "Quality Assurance Measures for Environmental Data" in Lead in the Marine Environment, M. Branica and Z. Konrad, Eds., Pergamon Press, Oxford, England (1980). 
b. Talks

Taylor, J. K., "Technical Activities of the NBS Center for Analytical Chemistry", Japan-U.S. Symposium on SRM's, Koriyama, Japan, october 7, 1979 .

Taylor, J. K., "Environmental Standard Reference Materials", Japan-U.S. Symposium on SRM's, Koriyama, Japan, October 8, 1979.

Taylor, J. K., "Quality Assurance Program for Analytical Chemistry", Japan-U.S. Symposium on SRM's, Koriyama, Japan, October 9, 1979.

Taylor, J. K., "The Role of Standard Reference Materials in Quality Assurance of Chemical Measurements", Tokyo University, Tokyo, Japan, October 11, 1979.

Taylor, J. K., "Traceability of Chemical Measurements to National Measurement Standards", Kyoto University, Kyoto, Japan, October 13, 1979.

Taylor, J. K., "Methods for Preparation of Standard Gas Mixtures", National Institute for Environmental Studies, Tsukuba, Japan, October 16, 1979.

Taylor, J. K., "Recent Advances in Electroanalytical Chemistry", National Institute of Metrology, Beijing, China, November 14, 1979.

Taylor, J. K., "Coulometric Analysis", National Institute of Metrology, Beijing, China, November 15, 1979.

Taylor, J. K., "Water Pollution Analysis", National Institute of Metrology, Beijing, China, November 16, 1979.

Taylor, J. K., "Air Pollution Analysis", National Institute of Metrology, Beijing, China, November 17, 1979.

Taylor, J. K., "Measurement and Analysis of Particulates", National Institute of Metrology, Beijing, China, November 19, 1979.

Taylor, J. K., "Microchemical Analysis", National Institute of Metrology, Beijing, China, November 20, 1979.

Taylor, J. K., "Quality Assurance and Its Role in the Management of Chemical Laboratories", National Institute of Metrology, Beijing, China, November 21, 1979.

Taylor, J. K., "Standard Methods and Standard Reference Materials", National Institute of Metrology, Beijing, China, November 22, 1979.

Taylor, J. K., "Quality Assurance of Chemical Measurements", Shanghai Academy of Sciences, Shanghai, China, November 27, 1979.

Taylor, J. K., "Standard Reference Materials", Shanghai Academy of Sciences, Shanghai, China, November 28, 1979. 
Taylor, J. K., "Water Pollution Analysis - Standardization of Methods SRM's", Kwangchow Academy of Sciences, Kwangchow, China, December 4, 1979.

Taylor, J. K., "The NBS Center for Analytical Chemistry", National Institute of Metrology, Beijing, China, December 7, 1979.

Taylor, J. K., "Principles of Quality Assurance Applied to Forensic Analysis", Federal Bureau of Investigation, Washington, D.C., December 14, 1979.

Taylor, J. K., "Quality Assurance of Chemical Measurements", VI International Symposium, "Chemistry of the Mediterranean", Rovinj, Yugoslavia, May 4, 1980.

\section{c. Committee Assignments}

John K. Taylor

Member, IS0/TC48, Laboratory Glassware and Related Apparatus ISO/TC48/SCl, Volumetric Glassware

ISO/TC146, Air Quality

ISO/TC146/SC3, Ambient Mea surements

Chairman, ISO/TCl47, Water Quality

Member, ISO/TC147/S.C7, Terminology

ISO/TC147/SC2, Chemical, Physical, Biochemical Methods of Analysis ISO/TC147/SC6, Sampling

ANSI TAG, ISO/TC147, Water Quality

ANSI TAG, ISO/TC172, Optical Instruments

ASTM D19, Water

Chairman, ASTM D1 9.01.07, International Standards

Member, ASTM D19.02, General Specificiations

ASTM D19.05, Inorganic Constituents

ASTM D1 9.07, Sediments

ASTM D22, Atmospheric Analysis

ASTM D22.04, Workplace Atmospheres

Vice Chairman, ASTM D22.05, Calibration

Member, ASTM D22.07, Precision and Accuracy

ASTM D22.09, USTAG to TC 146

ASTM E34, Occupational Safety and Health

ASTM E41, Laboratory Apparatus

ASTM E41.01, Glass and Plastic Apparatus

ASTM E41.06, Weighing Devices

ASTM E41.07, Microchemical Apparatus

Collaborative Testing Subcommittee - Standard Methods

Advisory Committee - CHEMTECH (ACS)

ACS Committee on Environmental Chemistry - Subcommittee on

Environmental Analytical Chemistry

NBS Museum Committee

NBS 80th Anniversary Celebration Committee

NBS Advisory Committee, State Measurement Needs Survey 


\section{National Environmental Specimen Bank \\ 1. Introduction}

In response to the growing concern for the potential dangers to human health and the environment by the increasing influx of man-made substances in our ecosystem, the U.S. Environmental Protection Agency (EPA) and the National Bureau of Standards (NBS) are currently studying the feasibility of establishing a National Environmental Specimen Bank (NESB). The program for such a Bank would incorporate a well-defined system of collection, analysis, and long-term storage of selected environmental samples to provide (1) real-time monitoring data for pollutant trend analysis and (2) properly collected and stored samples for retrospective analysis.

The National Bureau of Standards is currently involved in a five-year Pilot Environmental Specimen Bank Program which will provide actual working experience in all stages of the banking effort: specimen collection, processing, storage, analysis, and data management. The purposes of this pilot study are: (1) to develop analytical protocols for sampling and storage of four types of environmental samples, (2) to improve analytical methodologies for both trace elements and trace organics, (3) to evaluate the feasibility of long-term specimen storage at various conditions $\left(-25{ }^{\circ} \mathrm{C},-80^{\circ} \mathrm{C},-120^{\circ} \mathrm{C}\right.$, and freeze-dried at room temperature), and (4) to provide a "bank" of samples for retrospective analysis in future years as analytical methodologies improve. The experience gained in this pilot study will be used to evaluate the feasibility of the NESB Program. The rationale for this specimen banking program, a review of the NBS activities relating to this program, and an outline of the proposed five-year pilot specimen bank study at NBS have been described previously $[1,2]$. In these previous reports, the construction of the clean laboratory/storage facility was described and the preliminary plans for sample collection and storage were reviewed. During this initial year of the proposed five-year pilot study, the collection, processing, storage evaluation, and trace element analysis of the first environmental specimens, human livers, were implemented. Detailed reports $[1,3]$ are available which describe the pilot specimen bank program. Highlights from these reports are included here.

\section{Safety Requirements}

The handling of human liver samples carries the potential risk of exposure to infectious diseases, particularly hepatitis B virus or "serum hepatitis." As a result, certain precautions are necessary to insure protection of the analysts involved in sample processing and analysis.

To eliminate potentially infectious liver specimens from the specimen bank samples, a blood sample is removed from the donor at the time of autopsy to be used for hepatitis B screening. In addition, liver specimens from the right lobe are removed for preparation of histological slides. These slides are examined by the pathologists for evidence of 
infectious diseases. Liver samples are placed in temporary storage at liquid nitrogen temperatures at NBS until the results of the screening are received from the hopsitals supplying the livers.

A Safety Advisory Committee has been established to advise the Center for Analytical Chemistry concerning suitable safety precautions for the handling and processing of these liver samples at NBS. This committee consists of Michael Greene, M.D. (NBS physician), Robert Purce11, Ph.D. (Chief of Hepatitis Section, National Institutes of Health), and Tom Koch, Ph.D. (Department of Pathology, University of Maryland). Recommendations were made by the committee regarding procedures to safeguard personnel. The Safety Advisory Committee will be requested to review annually the procedures of the pilot specimen bank program.

\section{Collection of Liver Samples}

Contracts for procurement of liver samples were negotiated and awarded to three medical schools located in Baltimore, Maryland; Minneapolis, Minnesota; and Seattle, Washington. Each contractor is providing 100 liver samples this year for a total of 300 samples (see figure 1).

Due to the extremely low levels of trace elements and organic pollutants in human liver tissue, the analytical chemist must exercise extreme caution to avoid possible contamination of the sample. (The lack of contamination control has made existing banks of samples useless for general trace constituent analyses.) A detailed sampling protocol, designed from the viewpoint of analytical chemists, is utilized to minimize contamination. The written sampling protocol outlines in detail the case selection criteria and the steps to be followed by the contractor in obtaining a "valid" sample. The implementation of this protocol is a result of extensive interaction with NBS personnel and the contractors to insure the quality of the samples.

The individual collecting the liver samples uses (1) non-talced gloves, (2) a special titanium-bladed knife for bisecting the liver (to avoid contamination from other elements, i.e., $\mathrm{Cr}$, Ni, etc.), (3) Teflon sheets upon which the sample is placed, and (4) high-purity water to rinse the sample. The samples are then sealed in Teflon bags, frozen at liquid nitrogen temperature, and shipped to NBS. A data form, sent with each liver sample, contains information about the donor and specimen, e.g., date of birth, sex, residence, ethnic group, height, weight, smoker, occupation (if known), diagnosis of autopsy, date and time of death and autopsy, and weight of sample. Approximately 100 samples have been received and stored in the pilot bank facility at NBS.

The scheme for the collection and allocation of subsamples for storage evaluation are shown in figures 1 and 2 . The liver specimens are received as duplicate sections of the left lobe identified as sections "A" and "B". A1T "A" sections are placed in liquid nitrogen freezers for long-term storage, and the "B" sections are used for storage evaluation, 


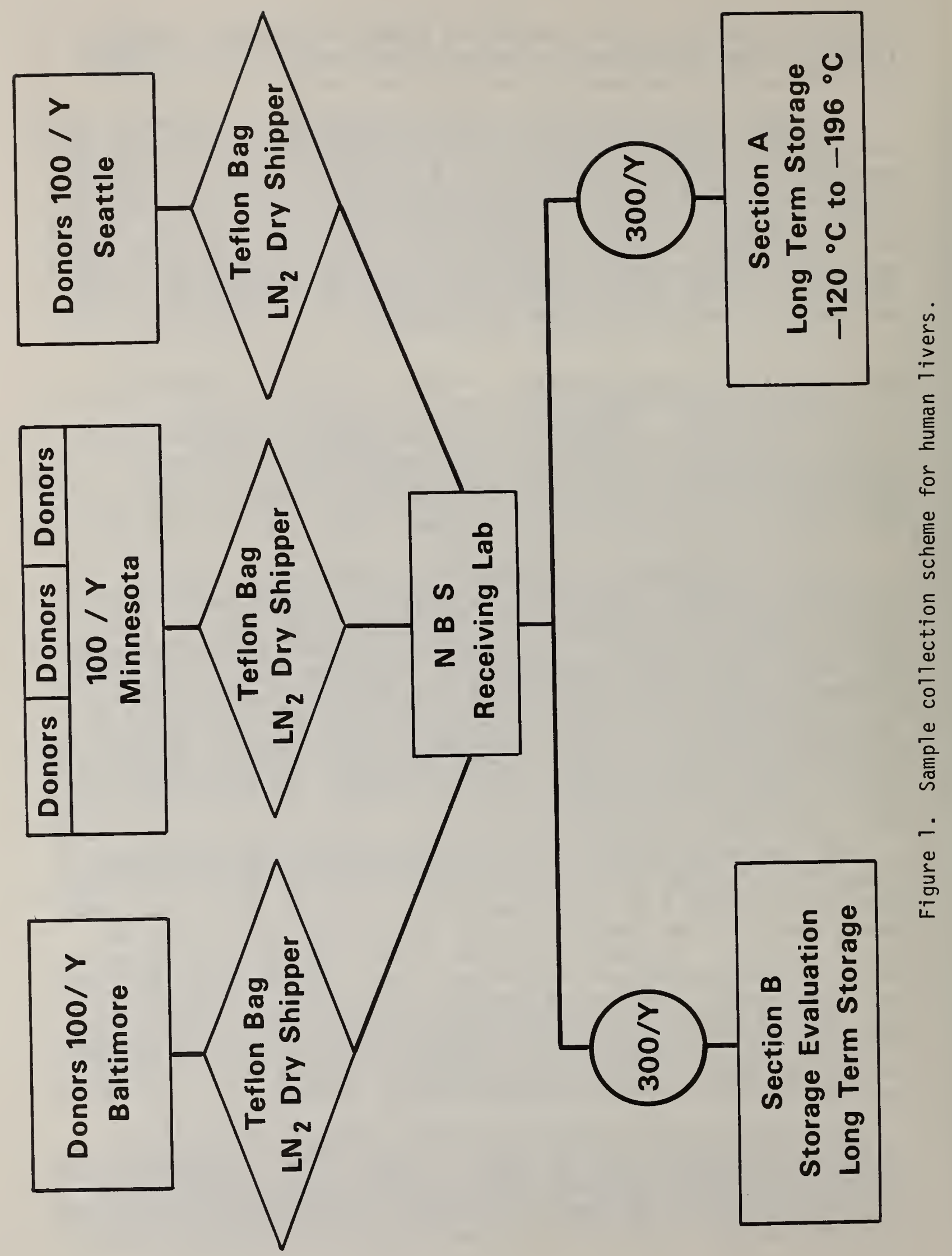




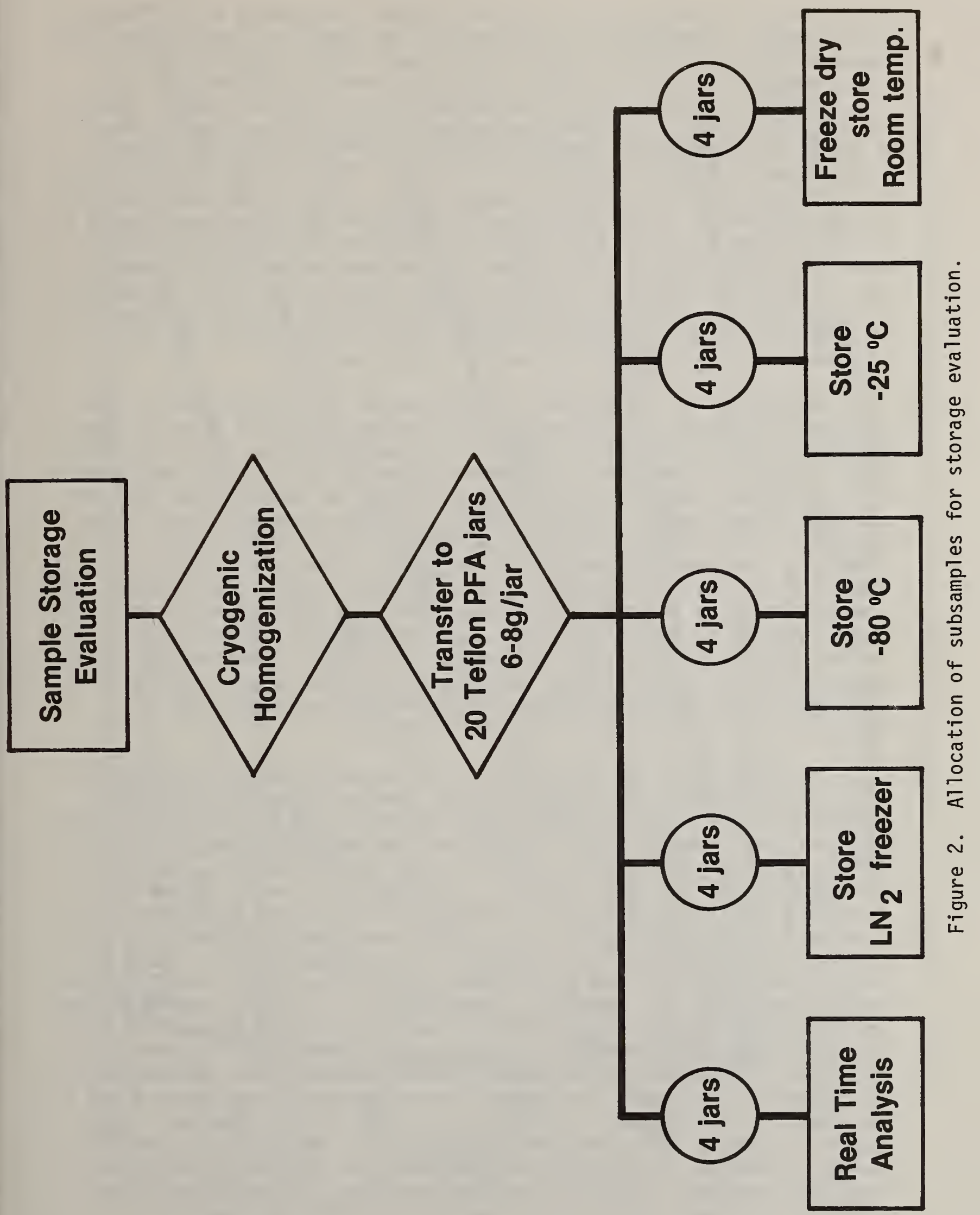


i.e., real-time analysis and long-term storage evaluation at different conditions. Approximately 10 percent of the samples will be homogenized using a cryogenic brittle fracture technique. This homogenization provides about 20 aliquots of $5-8 \mathrm{~g}$ which are stored in Teflon jars. To solve the question of appropriate temperature for sample storage, these sample aliquots will be stored under four different conditions: freezedried at room temperature, fresh frozen at $-25{ }^{\circ} \mathrm{C}$, at $-80^{\circ} \mathrm{C}$, and in liquid nitrogen vapor at $-120{ }^{\circ} \mathrm{C}$ to $-190{ }^{\circ} \mathrm{C}$. The concentrations of trace constituents found after storage under those conditions will be compared to the data found in the "real-time" analysis of each specimen (figure 2). The "A" sections are reference samples which may be used to re-evaluate results on a particular "B" section. At the end of the pilot program, the " $A$ " sections stored at 1 iquid nitrogen temperatures will represent a valuable bank of validated samples available to the scientific community. In addition, a large quantity of data, from the analyses of the " $B$ " sections, will be available on many of these samples.

The success of the NESB will be determined in a large part by the ability to preserve the integrity of the trace substances (i.e., organic, inorganic, and organometallic species) in samples during long-term storage. Changes in the forms and concentrations of the numerous environmentally important substances in specimens stored for extended periods may occur in several ways. Processes such as surface adsorption and sample degradation may reduce the concentrations of various components. In addition, continued biochemical and enzymatic activity may produce species which may not have been present in the original sample. On the other hand, contamination of the specimen fluids could lead to apparent increases in trace substances' concentrations. Superimposed on these processes are the factors which will affect the process rates, such as container material, contact time and area, storage temperature, $\mathrm{pH}$, and initial species concentration. All of these factors are important considerations in evaluating the suitability of long-term storage.

The probability for the discovery of changes in the concentrations of trace constituents during storage has been assessed by statistical treatment of the expected experimental parameters, e.g., number of specimens analyzed, homogeneity of subsamples, and analytical error ( $K$. $R$. Eberhardt and L. DeRobertis, Center for Applied Mathematics, Statistical Engineering Division, NBS). Figure 3 consists of a series of curves generated from the experimental parameters of 5 percent inhomogeneity and 5 percent analytical error. These curves determine the probability of detecting a particular percent change in concentration (e.g., 6 and 4 percent for curves 1.0 and 0.7 , respectively) for a given number of liver samples analyzed. For example, we would have a 98 percent probability of detecting a 6 percent change (Point A) and a 78 percent probability of detecting a 4 percent change in concentration (Point B) if 30 livers are analyzed. If the sample inhomogeneity or analytical error increased to 10 instead of 5 percent, the percent change detectable at Points $A$ and $B$ would be 12 and 8 percent, respectively. Based on these curves, the number of samples to be analyzed was set at 30 . Analyses of a smaller number of samples would significantly reduce the probabilities of detecting small changes, whereas increasing the number of samples analyzed would not increase greatly the probability of detecting changes. 


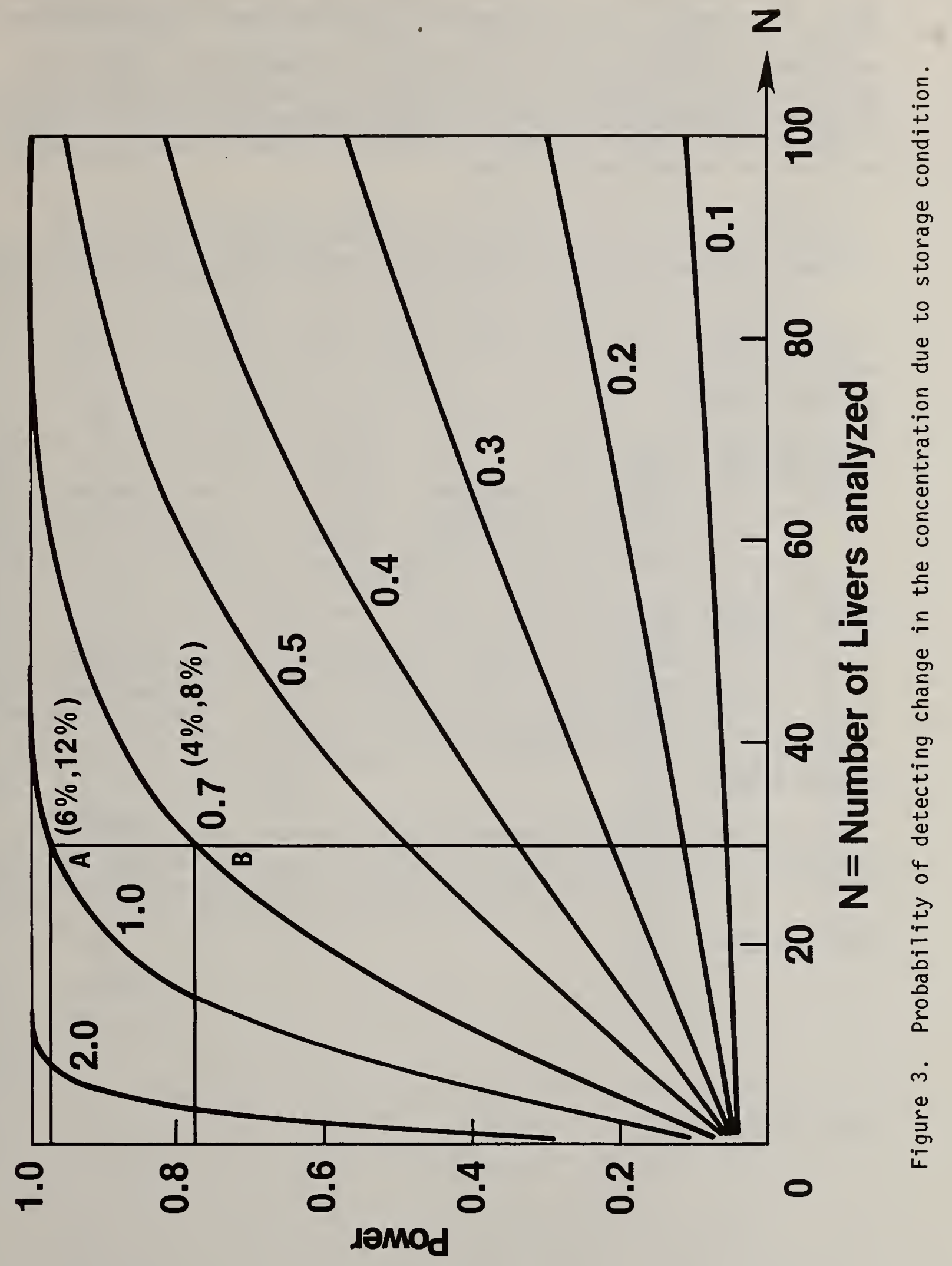


The 30 specimens used for the storage evaluation represent approximately 10 percent of the specimens collected per year. A major limitation to this number is the present analytical capacity. In order not to exceed the currently available analytical capability, most of the analytical work is deferred to the year 1984, when the program should have a larger analytical capacity. For the evaluation of the storage of human livers, Table 1 gives the analytical needs in terms of numbers of livers to be analyzed each year.

Table 1. Number of Livers to be Analyzed for Storage Evaluation Study.

\begin{tabular}{|c|c|c|}
\hline Year & & 80 \\
\hline Real Time Analys & sis & 36 \\
\hline 1 Year Storage & $\mathrm{LN}_{2}$ & \\
\hline & $-85^{\circ}{ }^{\circ} \mathrm{C}$ & Samples \\
\hline & $\begin{array}{c}-25{ }^{\circ} \mathrm{C} \\
\text { RT }\end{array}$ & from ' 80 \\
\hline
\end{tabular}

$$
\frac{82}{30}
$$$$
83
$$

2 Years Storage $\mathrm{LN}_{2}$

3 Years Storage $\mathrm{LN}_{2}$

$$
\begin{aligned}
& -85{ }^{\circ} \mathrm{C} \\
& -25{ }^{\circ} \mathrm{C} \\
& \mathrm{RT}
\end{aligned}
$$

4 Years Storage $\mathrm{LN}_{2}$

$$
\begin{aligned}
& -85{ }^{\circ} \mathrm{C} \\
& -25{ }^{\circ} \mathrm{C} \\
& R T
\end{aligned}
$$

Samples 


\section{Trace Element and Trace Organic Analysis}

The trace element analysis activities of the specimen bank project have concentrated on three main tasks this year: (1) development of a comprehensive analytical scheme, (2) evaluation of the sample collection protocols, and (3) initiation of the analysis of the first year's collected samples.

The current analytical protocol is directed toward the analysis of most of the first-priority elements proposed by the International Workshop on Monitoring Environmental Materials and Specimen Banking [4]. Additional elements occurring as biological major elements and trace elements in the samples are included in the protocol because their levels might supply information about the "normal" state of the specimen. The elements under consideration, their priority, and the analytical techniques to be used appear in Table 2. This scheme incorporates the NBS-CAC approach of multi-technique analysis for quality control. The first three techniques listed, i.e., atomic absorption spectroscopy (AAS), anodic stripping voltammetry (ASV) or linear sweep voltammetry (LSV), and instrumental neutron activation analysis (INAA), comprise the current routine analytical scheme. Isotope Dilution Mass Spectrometry (IDMS) and radiochemical neutron activation analysis (RNAA) will be performed on a limited number of samples to provide multi-technique quality control as well as to add data on el ements of special environmental interest, e.g., thallium. Prompt gamma activation analysis (PGAA) will be performed in collaboration with the University of Maryland, Division of Nuclear and Environmental Chemistry. Preliminary results from PGAA of samples of specimen bank livers appear promising for the elements indicated in Table 2, particularly for the major elements, $\mathrm{H}$, $\mathrm{C}$, and $\mathrm{N}$, and the trace element, $\mathrm{B}$. The determination of $\mathrm{H}, \mathrm{C}$, and $\mathrm{N}$ may be useful in the planned research on the most suitable weight basis, e.g., dry versus wet weight.

According to the proposed storage evaluation scheme, 36 livers are being analyzed during the first year of sample collection. These samples are being analyzed by AAS, voltammetry, INAA, and IDMS. Some preliminary results of liver analyses are shown in Table 3 . 
Table 2. Comprehensive Analytical Scheme for Trace Elements in Human Liver.

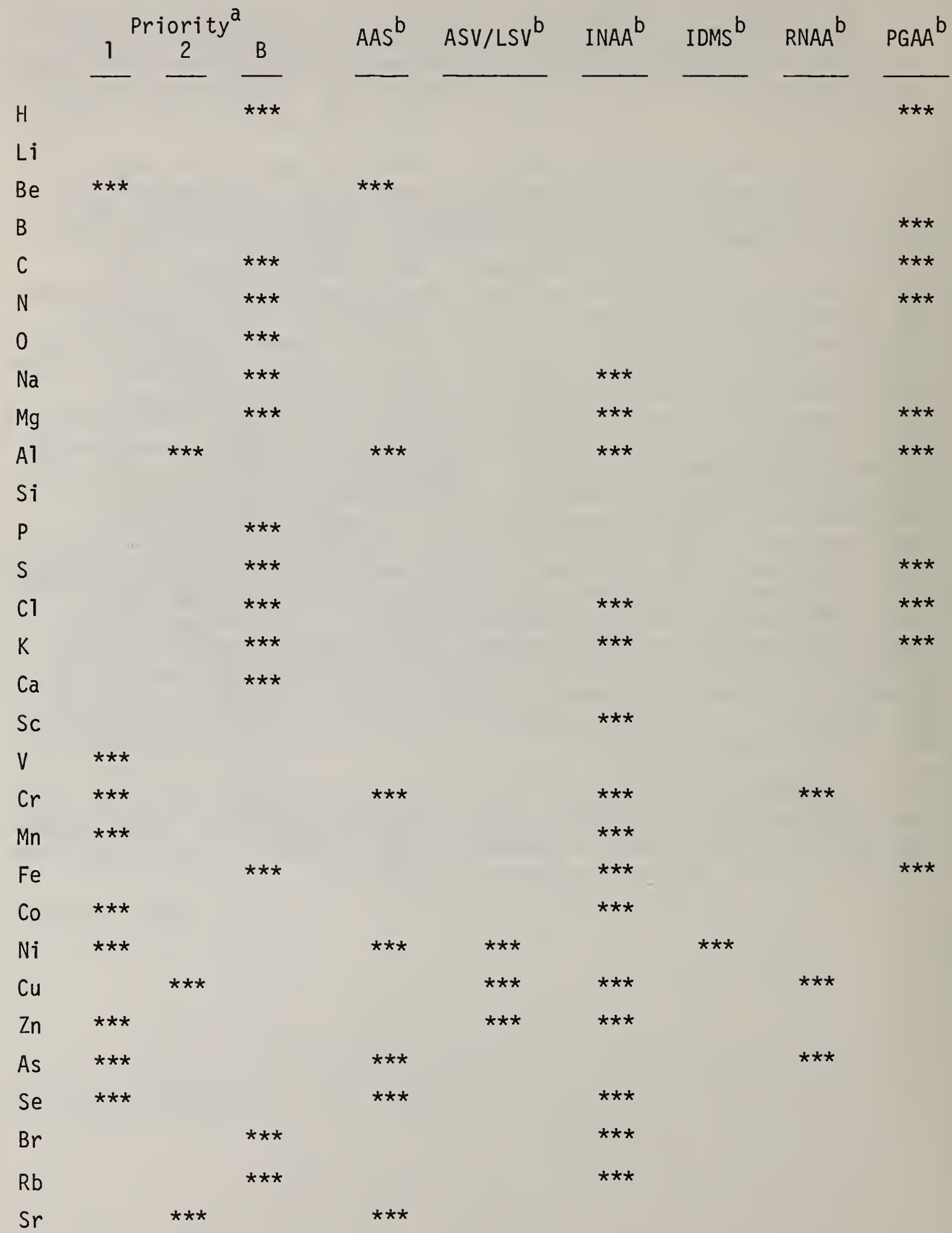


Table 2. Comprehensive Analytical Scheme for Trace Elements in Human Liver, continued.

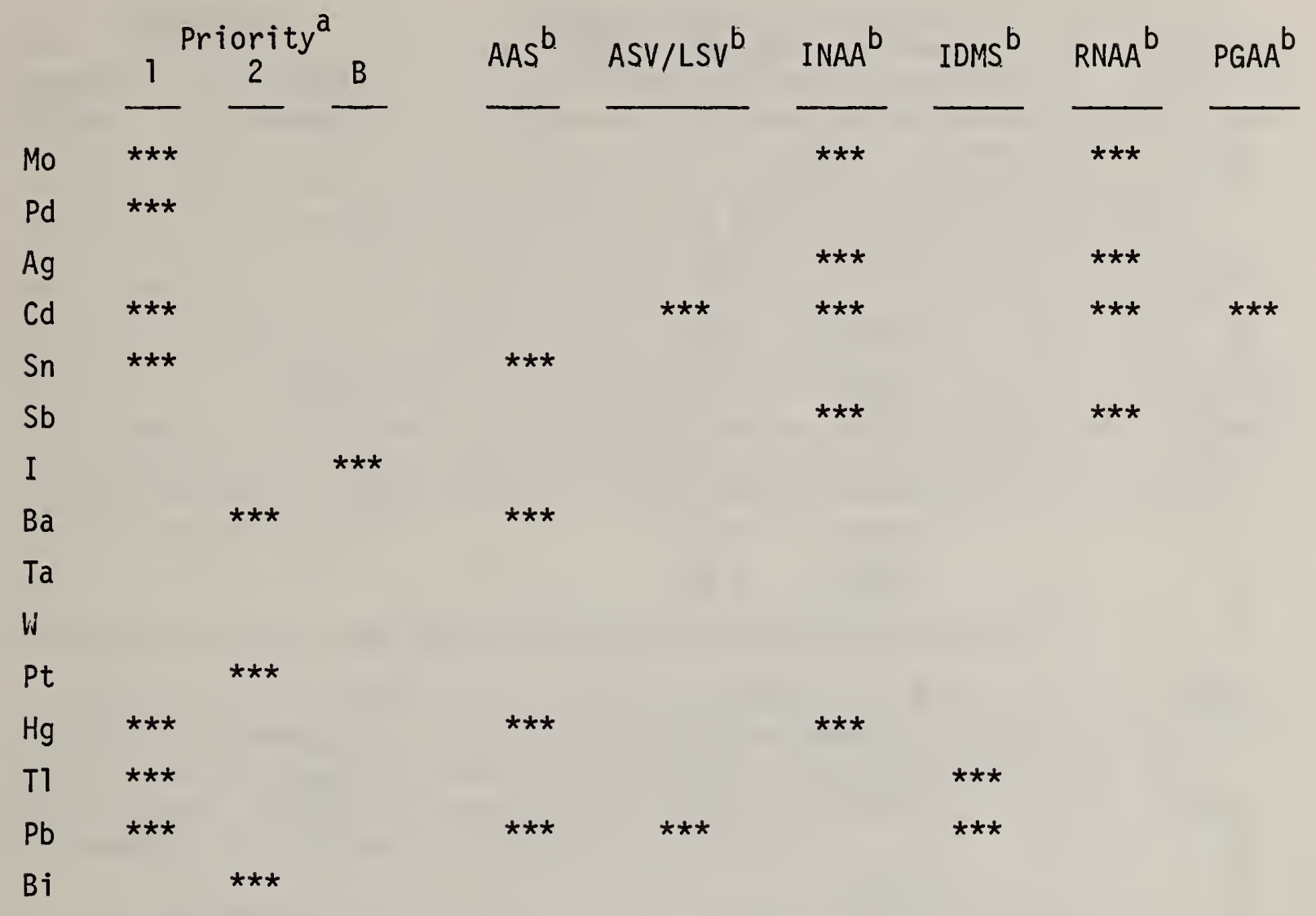

$\mathrm{a}_{1}=$ First priority el ements from "International Workshop on Monitoring Environmental Materials and Specimen Banking" held in Berlin, October 1978.

2 = Additional elements of environmental concern.

$B=$ Biological elements.

${ }_{\text {AAS }} \quad=$ atomic absorption spectroscopy;

ASV/LSV = anodic stripping voltammetry/linear sweep voltammetry;

INAA = instrumental neutron activation analysis;

IDMS = isotope dilution mass spectrometry;

RNAA = radiochemical neutron activation analysis;

PGAA = prompt gamma activation analysis. 
Table 3. Analysis of Trace Elements in Human Liver.

\begin{tabular}{|c|c|c|c|c|}
\hline \multirow{2}{*}{ El ement } & \multirow{2}{*}{\multicolumn{2}{|c|}{$\begin{array}{l}\text { Concentration Range }(\mu \mathrm{g} / \mathrm{g}) \\
\text { from the Literature }[5] \\
\text { for Human Livers }\end{array}$}} & \multicolumn{2}{|c|}{ NBS Results } \\
\hline & & & INAA $^{\mathrm{a}}$ & $\mathrm{ASV} / \mathrm{LSV}^{\mathrm{a}}$ \\
\hline $\mathrm{Be}$ & & VD & & \\
\hline $\mathrm{F}$ & 0.06 & $-\quad 1.4$ & & \\
\hline Al & 1.6 & $-\quad 2.6$ & & \\
\hline V & $<0.007$ & 0.09 & & \\
\hline $\mathrm{Cr}$ & 0.005 & 0.27 & 0.04 & \\
\hline $\mathrm{Mn}$ & 0.5 & $-\quad 1.9$ & & \\
\hline $\mathrm{Fe}$ & 70 & -210 & 154 & \\
\hline Co & 0.017 & $-\quad 0.16$ & 0.039 & \\
\hline $\mathrm{Ni}$ & 0.009 & 0.32 & & $<0.4$ \\
\hline $\mathrm{Cu}$ & 3.2 & $-\quad 14.7$ & 11.2 & 10.1 \\
\hline $\mathrm{Zn}$ & 31 & -80 & 63 & 64 \\
\hline As & 0.006 & 0.46 & 0.033 & \\
\hline $\mathrm{Se}$ & 0.097 & 0.68 & 0.35 & \\
\hline $\mathrm{Rb}$ & 7 & -12 & 3.7 & \\
\hline $\mathrm{Sr}$ & 0.01 & & & \\
\hline Mo & 0.4 & 1.6 & 1.0 & \\
\hline $\mathrm{Pd}$ & & VD & & \\
\hline $\mathrm{Ag}$ & 0.006 & 0.07 & 0.044 & \\
\hline $\mathrm{Cd}$ & 0.5 & 4.9 & 1.1 & 0.9 \\
\hline Sn & 0.08 & 0.65 & & \\
\hline $\mathrm{Sb}$ & 0.01 & & 0.007 & \\
\hline $\mathrm{Ba}$ & 0.01 & & & \\
\hline Pt & & ND & & \\
\hline $\mathrm{Hg}$ & 0.005 & 0.25 & + & \\
\hline $\mathrm{Tl}$ & 0.001 & 0.009 & & \\
\hline $\mathrm{Pb}$ & 0.8 & 2.3 & & 0.7 \\
\hline
\end{tabular}

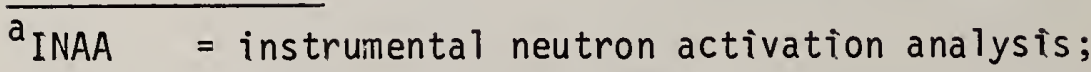
ASV/LSV = anodic stripping voltammetry/linear sweep voltammetry. Results are based on fresh weight. 
Analytical methods for the analysis of two classes of organic compounds, i.e., organochlorine pesticides and polycyclic aromatic hydrocarbons (PAH) are currently being developed. Preliminary results have been obtained using the following analytical procedures: (1) extraction with acetonitrile, (2) 1 iquid-liquid partition into hexane, (3) concentration by evaporation, (4) high-performance liquid chromatography (HPLC) to isolate the compounds of interest, and (5) gas chromatography (GC) analysis on capillary columns with selective electron capture detection. An HPLC method, using a chemically bonded amine column has been developed to isolate the organochlorine pesticides and $\mathrm{PAH}$ from interfering organic constituents. GC methods have been developed for the determination of the 16 pesticides on the priority pollutant list. Research is continuing on the methodology for quantitation of these compounds in liver samples in order to initiate real-time analyses for organics in 1981. Future plans include (1) the evaluation of homogeneity for organics, (2) the development of procedures for comparison of organochlorine content determined by GC and determined by neutron activation analysis, and (3) expansion of the pilot program to include development of analytical protocols for the mussel/ oyster samples.

\section{Interactions with National and International Programs}

Since 1975, the U.S. EPA and the Environmental Agency of the Federal Republic of Germany have been pursuing collaborative research in the area of the specimen bank program as part of a bilateral agreement on the environment. Recently, specimen banking was incorporated into a similar agreement with the People's Republic of China.

In November 1979, NBS, in conjunction with EPA, hosted the third annual meeting as part of the Bilateral Agreement on the Environment with representatives of the Federal Republic of Germany. This meeting addressed in general the environmental specimen bank programs and specifically, the implementation of pilot programs presently underway in both countries. Construction of the German specimen bank facility is expected to be completed in early 1981.

In May 1980, NBS scientists were invited to participate in a "Workshop on Research Needed to Improve Data on Mineral Content of Human Tissues," sponsored by the American Institute of Nutrition and funded by, the National Science Foundation. In an editorial in Analytical Chemistry 52, 1393 (1980) concerning this workshop, Dr. George H. Morrison, editor of Analytical Chemistry and Professor of Chemistry at Cornell University, commented on the NBS Pi lot study:

"Of particular interest is the recent establishment of a pilot environmental specimen bank at the National Bureau of Standards in cooperation with the Environmental Protection Agency (Anal. Chem. $1980,52,887 \mathrm{~A}$ ). Initial efforts are concerned with the gathering, processing, and storage of human liver samples. The goal of the specimen bank program is to develop analytical protocols and measurement methods, and to evaluate the various 
storage and sample preparation techniques for their effects on the samples. This pilot study, limited to environmental considerations of one human tissue, is a good beginning to the broader problem of the mineral content of human tissues."

We have recently initiated interactions with the "Mussel Watch Program," coordinated by Dr. Donald Phelps at EPA/Narragansett, Rhode Island, to coordinate protocol and analytical methods development for the mussel/oyster samples. The "Mussel Watch Program" will help provide samples for the pilot banking program.

\section{References}

[1] Gills, T. E., Rook, H. L., and Durst, R. A., The National Environmental Specimen Bank Research Program for Sampling, Storage, and Analysis, EPA-600/1-79-017, U.S. Environmental Protection Agency (1979).

[2] NBSIR 80-1995, Technical Activities 1979, Center for Analytical Chemistry (1979).

[3] Harrison, S. H., Zeisler, R., and Wise, S. A., Pilot Program for the National Environmental Specimen Bank--Phase I, to be published as an NBS Technical Note.

[4] Selection of Pollutants and Pollutant Categories of Interest for Monitoring Environmental Materials, in Monitoring Environmental Materials and Specimen Banking, N. P. Luepke, ed., Martinus Nijoff Publishers, The Hague, 22 (1979).

[5] Iyengar, G. V., Kollmer, W. E., and Bowers, H. J. M., The El emental Composition of Human Tissue and Body Fluids, Verlag Chemie, Weinheim--New York (1978).

S. A. Wise, Project Manager, K. A. Fitzpatrick, R. R. Greenberg,

S. H. Harrison, W. F. Kline, J. K. Langland, E. J. Maienthal, R. Zeisler 


\section{Service Analysis}

An important function of the Center for Analytical Chemistry is the provisions of consultation, advice, and expert chemical analyses on the many types of diverse materials that are utilized in the research programs at NBS. This service is also available to other Federal, state, and local agencies when it is in the instances, where the CAC has a unique analytical capability, thus some may be extended to the private sector.

In 1980, the staff of the Center for Analytical Chemistry performed 147 service analyses. Of this number, 125 were performed on a cost reimbursable basis and, for the first time, because of a new funding structure within the Center, 22 were performed on a no cost basis. Among the three categories of customers, the largest user was the NBS staff with 64 requests $(\$ 108,000)$, followed by private industries, 52 requests $(\$ 33,000)$ and, thirdly, other federa1, state, and county agencies, 31 requests $(\$ 39,000)$.

On the whole, the year was typified by an increased number of requests for short and rapid turn-around analyses. The two most frequent requests were for the identification of suspected asbestos-containing materials and for the recalibration of glass filters used as standards for checking the transmittance accuracy of visible spectrophotometers. Among the more interesting analyses were the following studies:

- Investigation of abnormally high incidence of broken hypodermic needles in products from one pork processing plant (U.S. Department of Agriculture)

Examination of broken needles supplied by this plant yielded no explanation for their abnormally high occurrence. Subsequent physical and chemical analyses of new needles from the six U.S. and foreign manufacturers that could be identified showed that they were not distinguishable and thus any attempt to associate the broken needles with a particular source was not possible. On examining more closely the details of the plant's history (it did not do its own slaughtering, had considerable worker unrest and, generally, was reluctant to comply with Department of Agriculture recommendations), it became apparent that the broken needle problem could be possibly attributed to internal strife. To pursue this theory it was requested that whenever a broken needle was found, the pork sample in which it was found should also be submitted for analysis. From the trace chromium and nickel data, a reasonable estimate of the time the needle had been in the sample could be made. Ironically, since requesting tissue samples and explaining their intended use, the discovery of additional broken needles has suddenly ceased.

- Materials failure of steel bars used in construction of new jail facility (District of Columbia Government) 
Spectrographic analyses and hardness measurements showed that the bars used in construction of this facility did not meet specifications.

- Characterization of ink wastes for compliance with the new Environmental Protection Agency's hazardous waste regulations (U.S. Bureau of Engraving and Printing)

Atomic absorption analyses of leachates obtained from application of EPA's extraction procedure to wastes produced in the various printing and plating processes indicated that lead and chromium were abnormally high in two operations. Additional samples from these two sites have been requested for a more thorough evaluation.

- Analysis of a large number of culture media for trace concentrations of calcium (National Institutes of Health)

In the growing of mouse epidermal cells the calcium concentration in the culture media must be kept below about $20 \mathrm{ppm}$ in order to prevent the multi-layering of cells. In a low calcium serum culture medium, the cells will grow in a uniform layer indefinitely. Using repetitive optical scanning for background correction in conjunction with flame emission analysis, NBS has, periodically, accurately monitored the efficiency of ion exchange procedure used for calcium removal.

- Determination of "age" of wire recovered from tropical environment (National Security Agency)

Electron probe and spectrographic data obtained on the corrosion products permitted the NBS Chemical Stability and Corrosion Division to estimate the time that the wire had been in service to be $18 \pm 6$ months.

- "Soft" spots in aluminum alloy plate (National Aeronautics and Space Administration)

As a part of a larger study being carried out by the Center for Materials Science on possible soft spots in aluminum alloys, analyses were performed for copper, manganese, and iron in samples taken from various locations across the face of a 2219 aluminum alloy ingot. These analyses confirmed that part of the variations in the mechanical properties across the finished alloy plate is caused by significant variation in the concentrations of these alloying elements. Subsequently, CMS has determined that while macrosegregation of these alloying elements cannot be completely eliminated by chill face scalping and subsequent thermomechanical treatment, good scalping practice should reduce significantly the deterioration in mechanical properties.

R. H. Burke 


\section{E. New Competence Building Programs - FY'81}

1. Analysis of Ionization Mechanisms and Ion Structures

NBS currently occupies a leadership position in the utilization of analytical mass spectrometry for the qualitative and quantitative analysis of "real-world" samples. Most current researchers in mass spectrometry accept hypothesized ionization mechanisms and assumed structures of ions on faith, with quantitative internal standards being extensively used in "correcting" for a lack of basic understanding. Recent advances in mass spectrometer instrumentation, chemical enhancement of ionization processes, and laser physics make this an opportune time to further our basic understanding of the ionization process and ion structures. It is the goal of this competence building program to utilize these recent advances to develop a theoretical background on which analytical measurements can be based, and in so doing, maintain and build on the NBS leadership position in analytical mass spectrometry.

The main scientific and technical thrusts are centered about the use of chemical and physical means for probing ionization mechanisms/ enhancement and ion structures.

- Chemical enhancement of the ionization process will be examined as a probe of species in the ionization region of the mass spectrometer and collisional activation of ions with reactive gases will be examined as a probe of the structure of organic ions.

- Laser enhancement of the ionization process will be examined as a sensitive and selective probe of species in the ionization region, and laser induced metastable ion generation will be examined as a selective probe of the structure of ground-state and excited organic ions.

As one output of this program, experiments have already been designed which would examine the "charge localization" concepts, a basic hypothesis underlying our current understanding of fragmentation processes in mass spectrometry.

NBS progress in the research discussed above will have direct application to other research in the Center for Analytical Chemistry (e.g., emission spectroscopy) and will allow a more effective attack on measurement problems being faced by NBS Programs and other agencies requesting CAC analytical expertise.

Industry has become increasingly concerned with measurements of trace substances in complex matrices. This concern has been increased by considerable recent legislation which mandates regulation with respect to nutritional labeling, good laboratory practices, toxic substances and the environment. The proper and timely response to these concerns, as well as the enforcement of all such legislation and regulations are dependent upon continuing advances in the state-of-the-art of analytical techniques such as those discussed above. 


\section{Development of Methods for Compositional Mapping}

NBS has played a major role in the development of analytical methods based on excitation with particle beams of electrons, ions, photons, and neutrons. An important extension of these techniques for the characterization of samples is the preparation of "area maps" which plot the lateral distribution of characteristic signals, and "depth profiles" which map the distribution in depth of elemental or molecular constituents. While these area and depth maps can give a qualitative sense of the spatial distribution of constituents of interest, extension of the mapping techniques to yield quantitative compositional maps raises exciting experimental and theoretical challenges. Recent advances at NBS and el sewhere suggest that this is an opportune time to pursue the development of several methods of compositional mapping. The fundamental scientific problems underlying these techniques can be uniquely attacked at NBS because of the diversity of existing resources combined with the additions suggested in this proposal. It is the goal of this program to develop the theoretical and experimental bases necessary for the production of quantitative compositional maps by electron, ion, and neutron beam techniques. Further investigations will be made to evaluate the possibilities of selective organic compound mapping by photon excitation.

The main theoretical and experimental approaches can be divided into three sections:

- Lateral compositional mapping by electron beam x-ray microanalysis requires modeling of the electron-specimen interactions, particularly for the important class of rough specimens, and development of algorithms for rapid calculation. Complementary experimental work will be performed to develop new systems for rapid spectral acquisition.

- Lateral and depth compositional mapping by secondary ion mass spectrometry (ion microprobe) requires fundamental theoretical studies on the mechanisms of secondary ion formation and on the modification of secondary ion intensities by chemical state. Experimental studies will be conducted on techniques of rapid spectral acquisition and processing by computer control of the mass spectrometer.

- Depth compositional mapping by neutron irradiation requires development of the theoretical basis for quantification through accurate modeling and for unfolding the charged-particle energy spectrum. Experimental measurements are needed to determine the matrix dependency of the charged-particle stopping power and to improve depth resolution and elemental sensitivity. Upon establishment of this knowledge base, the techniques can be applied to the measurement of interesting new isotopes, e.g., ${ }^{26} \mathrm{Al}$. 
The development of quantitative compositional mapping will aid on-going research programs in CAC and el sewhere at NBS, as well as otheragency sponsored research programs, for example the AFTAC program on the characterization of particulates and the DOE project on the analys is of fusion reactor first-wall reactions. The specific measurement problems which can be addressed by these techniques include the characterization of metals, ceramics, semiconductor materials, and particulates, analysis. The behavior of many systems of profound interest to the physical and biological sciences, as well as many technological processes, are controlled by compositional variations on a spatial scale of 1 micrometer or less, and therefore, quantitative compositional mapping techniques can have great impact across a diverse range of scientific and engineering endeavors. 


\section{F. Instrument Development Group}

\section{Group Overview}

The main objective of this group is the development of new approaches to performing accurate chemical analyses. The group is working in two general areas: (1) investigating the use of lasers for chemical analysis; and (2) providing assistance to the Center staff on matters of instrument design, computer control, and processing of data.

James R. DeVoe

\section{a. Laser Project Overview}

Laser sources, with their high power, coherence, and spectral purity, are being used increasingly in analytical chemistry both in spectroscopy and in other applications. The rapid advances in laser technology and applications in physics and other branches of chemistry require research capabilities within the Center both to contribute to the advances and to effectively capitalize on developments elsewhere. The research in laser chemistry is threefold: basic and applied research in laser enhanced ionization; collaboration within the Center on the use of lasers in analytical applications; and collaborative efforts with other Centers when their work has potential analytical application.

Several investigations were completed during this year. Although no LEI signal was observed using a spark discharge several other interesting results were observed and were recently published. Multi-photon ionization of organic molecules has been completed and is reported below in the Technical Accomplishments section. Considerable progress has been made on the mechanism of LEI in flames. The inoperability of lasers has limited progress in certain areas. We have been unable to complete our semiconductor diode laser project because of a succession of diode failures. The stepwise multi-photon LEI in flames was not begun this year because of an inoperable flashlamp pumped system. Recently, we have purchased the beginning of a laser system that should perform adequately.

Our plans for LEI are to continue to study the fundamental processes for signal production and collection. These studies have already led to significant changes in electrode design and photo-excitation techniques. We have established that the basic principles of the quantitative analysis are sound and that the potential accuracy is high because of the interference-free nature of the spectroscopy. However, the technique is not immune to the interferences generally encountered in solutions that are aspirated into flames. For this reason we are interested in investigating the technique in other atom reservoirs, such as the solution aspirated into an inductively coupled plasma, hollow cathodes, various types of 
sparks, and totally new designs that involve combinations of techniques. Each of these areas, however, involves many man-years of effort. We plan to collaborate as much as possible with outside laboratories in this area.

Our long term plan is to develop the LEI in flames to a point where a quantitative analytical system can be assembled. At this point in our research, the "system" has been changing too rapidly to warrant assembly of the quantitative system. If a quantitative system can be assembled within the next two years, many types of real samples will be run in order to evaluate the effectiveness of LEI.

Several of our earlier investigations have led to new projects that are to be conducted in collaboration with staff in the various divisions. For example, the multi-photon ionization of organic molecules has led to a series of projects as part of competence building with the Organic Analytical Research Division. Other projects include laser assisted hot filament ionization and IR semiconductor diode absorption studies in the Inorganic Analytical Research Division and the Gas and Particulate Science Division, respectively.

Thomas C. O'Haver, Robert L. Sams, Richard Simon, John C. Travis, Gregory C. Turk

\section{b. Instrumentation Project}

An important contribution to the progress of research and development activities is technical support in electronic, mechanical, automation and computer system design. Support is being provided in five major areas: research involving the use of a new electronic design for current analytical instrumentation; the interaction of computer systems with analytical instrumenation; the design of mechanical components; the repair of all types of electronic and electromechanical equipment; and the utilization and/or lending of special test equipment for various experiments conducted within the Center.

There has been increased collaboration and interaction with scientists outside of the Center. Specifically, work is in progress or planned with staff in the Center for Materials Sciences, and the Center for Thermodynamics and Molecular Sciences on projects involving use of computers for data collection and processing. In addition, we have participated on a number of committees related to the use of computers at NBS (see appropriate sections below).

Andrew Bea1s, Lisa Bergen, John K. Langland, Robert McDiffett, Scott Mund, Fillmer C. Ruegg, Ronald W. Shideler 
2. Selected Technical Accomplishments

a. Studies of the Charge-Collection Mechanism in Laser Enhanced Ionization Spectrometry

The use of laser enhanced ionization (LEI) in flames for chemical anaTys is was proposed by us in 1976 [1], and has been the subject of a number of papers since then [2-8]. The principle behind the method, also known as the opto-galvanic effect, is that the volume ionization rate of an ionizing medium (such as a flame or discharge) is changed when a Taser is used to significantly perturb the electronic state population distribution of a species present in the medium [9-12]. This perturbation is observed experimental1y as a change in a current passed through the flame using external electrodes.

During the past year research was performed in collaboration with P. K. Schenck, of the Chemical Stability and Corrosion Division (561), CMS. The object of the research was to demonstrate the consistency of the spatial and temporal characteristics of the laser enhanced ionization signal with fundamental theoretical expectations based on a one-dimensional mode1. Three distinct types of experiments were performed:

(1) Voltage/current (V/I) characteristic curves were obtained for a variety of aspirated solutions, with and without laser irradiation. These studies revealed the response of the bulk electrical properties of the $\mathrm{H}_{2} / \mathrm{a}$ ir flame - the volume ionization rate, the positive ion space charge, and the electrical potential and field distribution - to changes in the sampled solution and laser excitation. At low applied voltages $\left(V_{a p p} \lesssim 200 \mathrm{~V}\right.$ ) the $2 \mathrm{~mm}$ diameter 1 aser beam appeared to increase the ionization rate throughout the $\sim 1 \mathrm{~cm}$ wide $\mathrm{flame}$, indicating a significant role for ion diffusion.

(2) Signal rise and fall time were measured as a function of electrode potential difference. In the high voltage limit $\left(V_{a p p} \gtrsim 200 \mathrm{~V}\right)$ the rise and fall times were found to obey the expected correlation with ion mobility, electrode separation, and applied voltage. The total charge arriving at the electrode (the integral under the current probe) was found to be independent of voltage. This fact is taken to indicate 100 percent efficiency in ion collection, since any postulated ion loss mechanisms (recombination, volume replacement, etc.) should be more effective for long ion extraction times (lower voltages) than short extraction times (higher voltages). At voltages $\lesssim 200 \mathrm{~V}$ the signal deteriorates due to space charge shielding of the electric field.

(3) One dimensional ion imaging was employed to profile the flame ionization rate as a function of height above the burner head. To the best of our knowledge, this is a new technique, and a patent search is in progress. The imaging is accomplished with the electrode configuration shown in figure 7 , in which the smal1, moveable probe rod intercepts a "slice" of the ions being transported by the well-defined field of the 


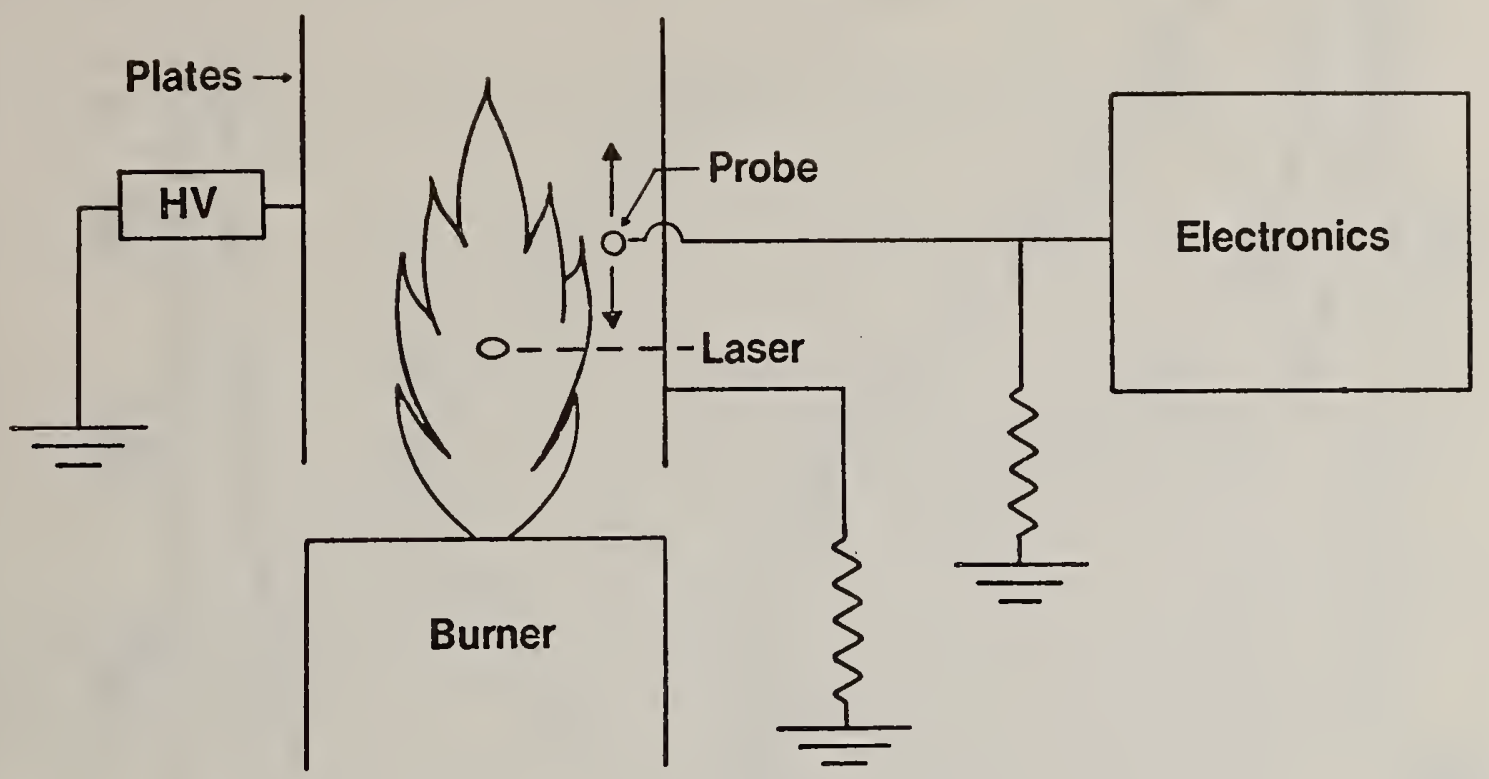

Figure 1. Apparatus for ion imaging.

parallel plates. Figure 2 shows a profile of the ionization rate taken with the system for an aspirated, $1 \mathrm{ppm} \mathrm{Na}$ solution, and $15 \mathrm{~mW}$ of laser excitation of the $589.0 \mathrm{~nm}$ transition. The ionization rate is seen to diminish smoothly from the reaction zone upward, with the pronounced exception of the "bulge" at $19 \mathrm{~mm}$ owing to laser excitation. Use of the full available $200 \mathrm{~mW}$ of laser power reduces the background component proportionally. By chopping the laser and using synchronous detection, the background component may be eliminated altogether, leaving only the laser-produced ion image. Such images are shown in figure 3 for two magnitudes and signs of high voltage applied to the HV electrode. For large potential drops, the image is sharp, and centered at the laser position. For low potential drops, the ion image spreads by diffusion and coulometric repulsion, and moves in the direction of the flame velocity. The collection of electrons (-HV) or positive ions (+HV) yields shape differences resulting from the vast difference in mass. The ion images confirm that ion losses to diffusion and flow removal are trivial at high voltages, again supporting a postulate of 100 percent efficient ion collection.

These experiments demonstrate significant agreement with theory illustrating the relative roles of the volume ionization rate, applied voltage and resulting current, flame and cold-air ion mobilities, electric field strength, laser power, diffusion, and electro-static repulsion. Several conclusions stated in the manuscript [13] resulting from the research are:

1) The results imply $\sim 100$ percent ion collection efficiency for high applied potentials; 


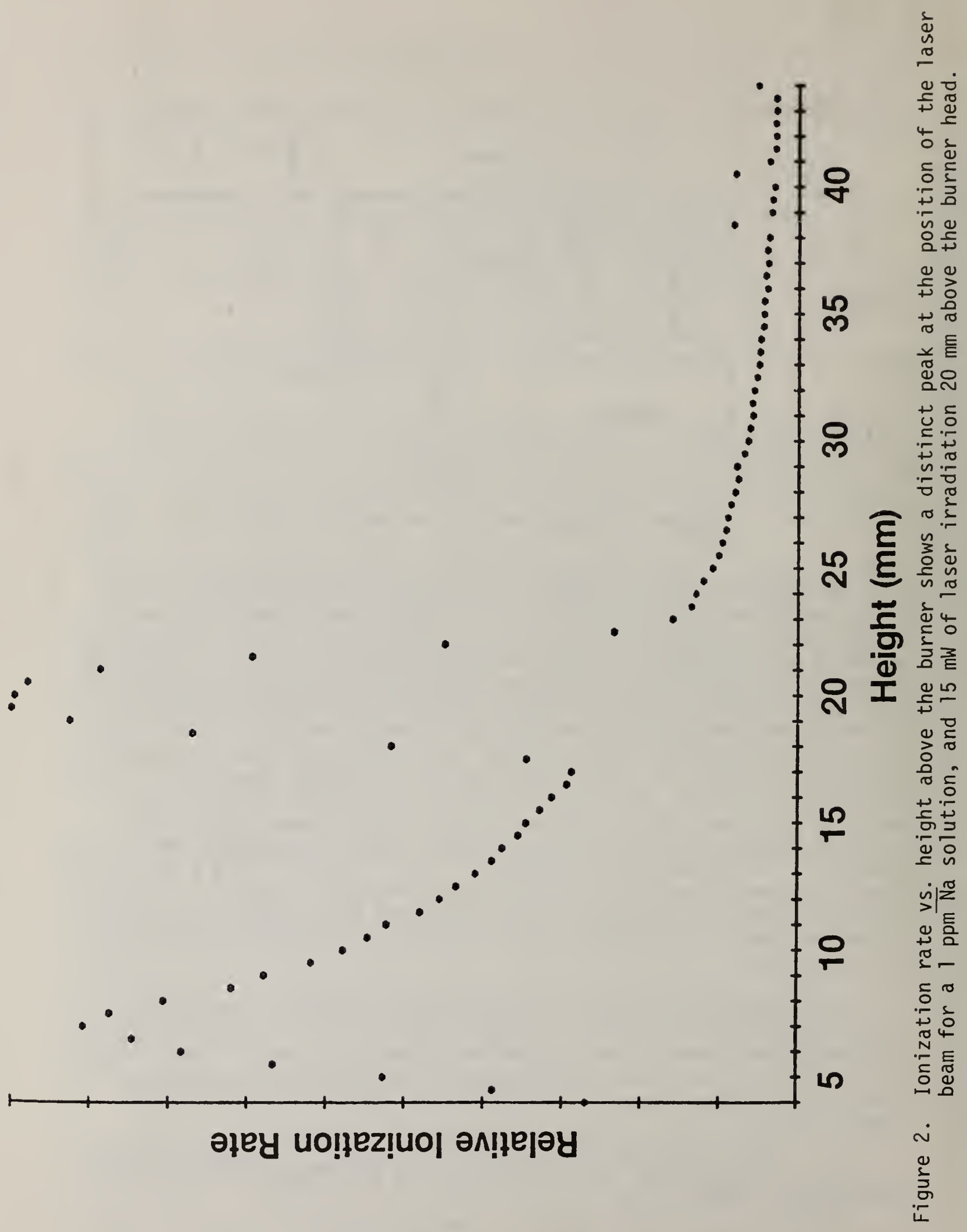




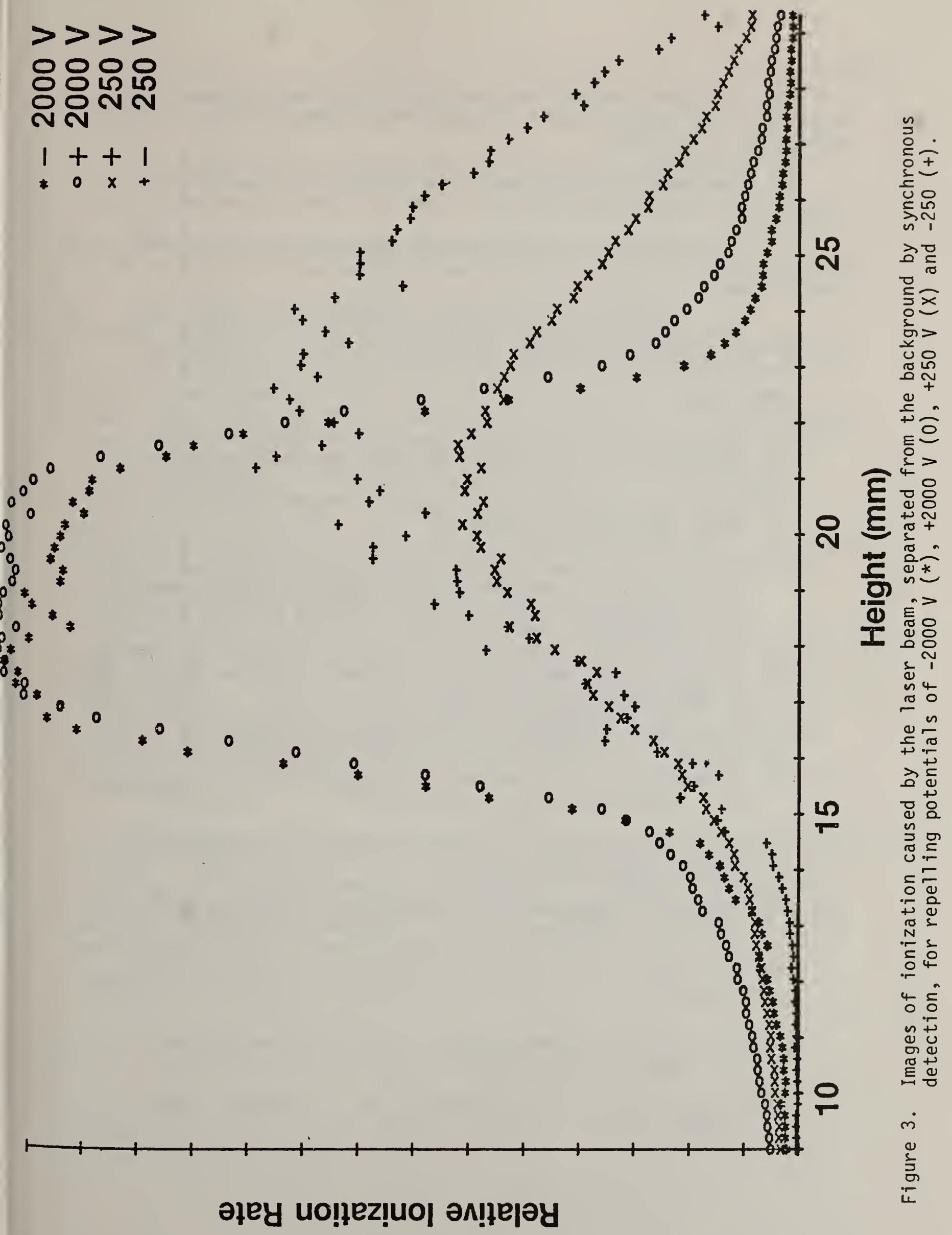


2) The steady-state excess ion distribution becomes considerably larger than the laser-irradiated volume for voltages well below electrical saturation of the flame;

3) The one-dimensional spatial ion-imaging technique should be a useful tool for combustion research - with or without laser irradiation;

4) Laser enhanced ionization is an attractive means of selectively increasing the ionization rate at a well defined position and time in a seeded flame;

5) The concepts developed should be useful in the further refinement of the analytical application of LEI.

References

[1] Green, R. B., Keller, R. A., Schenck, P. K., Travis, J. C., and Luther, G. G., J. Am. Chem. Soc. 98,8517 (1976).

[2] Turk. G. C., Travis, J. C., Devoe, J. R., and O'Haver, T. C., Anal. Chem. 50, 817 (1978).

[3] Travis, J. C., Turk, G. C., and Green, R. B., Chapter 6 in New Applications of Lasers to Chemistry, G. Hieftje, ed., ACS Symposium Series 85 , American Chemical Society, Washington, D.C., 91 (1978).

[4] Schenck, P. K., Mallard, W. G., Travis, J. C., and Smyth, K. C., J. Chem. Phys. 69, 5147 (1978).

[5] Turk, G. C., PhD Dissertation, University of Maryland (1978).

[6] Gonchakov, A. S., Zorov, N. B., Kuzyakov, Yu. Ya., and Mateev, 0. I., Anal. Lett. 12A, 1037 (1979).

[7] Turk, G. C., Travis, J. C., DeVoe, J. R., and O'Haver, T. C., Anal. Chem. 51, 1890 (1979).

[8] Turk, G. C., Mallard, W. G., Schenck, P. K., and Smyth, K. C., Ana l. Chem. 57, 2408 (1979).

[9] Van Dijk, C. A., PhD Thesis, University of Utrecht, The Netherlands (1978).

[10] Travis, J. C., Schenck, P. K., Turk, G. C., and Mallard, W. G., Anal. Chem. 51, 1516 (1979).

[11] Van Dijk, C. A., and Alkemade, C. Th. J., Combust. Flame 38,37 (1980). 
[12] Smyth, K. C., Schenck, P. K., and Mallard, W. G., to be published in the Proceedings of the American Chemical Society Symposium on Laser Probes of Combustion Chemistry.

[13] Schenck, P. K., Travis, J. C., Turk, G. C., and 0'Haver, T. C., submitted to the NBS Washington Editorial Review Board for approval.

J. C. Travis, G. C. Turk, T. C. O'Haver

\section{b. Atomic Resonance-Line Lasers for Atomic Spectrometry}

A new class of lasers has been developed at the Lincoln Laboratory of MIT which shows much promise as light sources for analytical spectroscopic measurements [1-3]. These lasers are unique in that they produce laser light at fixed frequencies corresponding to atomic resonance lines. Other fixed frequency atomic lasers (i.e. HeNe, $\mathrm{Cu}, \mathrm{Pb}, \mathrm{Hg}$ ) yield only nonresonance (excited state to excited state) transitions, which produce wavelengths of little use to the analytical spectroscopist. With the purpose of demonstrating the feasibility of such lasers for analytical spectroscopy a joint experiment was performed with D. J. Ehrlich and R. M. Osgood, Jr. at the Lincoln Laboratories, MIT. The new lasers were utilized as excitation sources for trace metal analysis via laser enhanced ionization (LEI) spectrometry.

The principle behind the atomic resonance laser (ARL) is the direct population of metal atom excited states through UV photodissociation of a metal halide, thereby producing an initial population inversion in the atomic species. The ARL itself is very simple, consisting of a $10 \mathrm{~cm} \times$ $2.2 \mathrm{~cm}$ diameter spectrophotometer cel1, evacuated except for a small amount of the appropriate metal halide and resulting vapor. Photodissociation of the halide is accomplished by optical pumping with an excimer laser. To date, lasers have been constructed for $\mathrm{Na}, \mathrm{K}, \mathrm{Rb}, \mathrm{Cs}$, $\mathrm{Tl}$, In, Ga, and $\mathrm{Al}$.

Laser enhanced ionization signals of thallium and sodium were studied. using $\mathrm{TlI}$ and $\mathrm{NaI}$ lasers. Partial energy level diagrams for $\mathrm{Tl}$ and $\mathrm{Na}$ are given in figure 4, showing the origin of the atomic laser lines for the two lasers. Table 1 summarizes the operating characteristics of the two lasers and the LEI analytical results. Excellent detection limits were achieved with a linear dynamic range of $10^{4}$. 


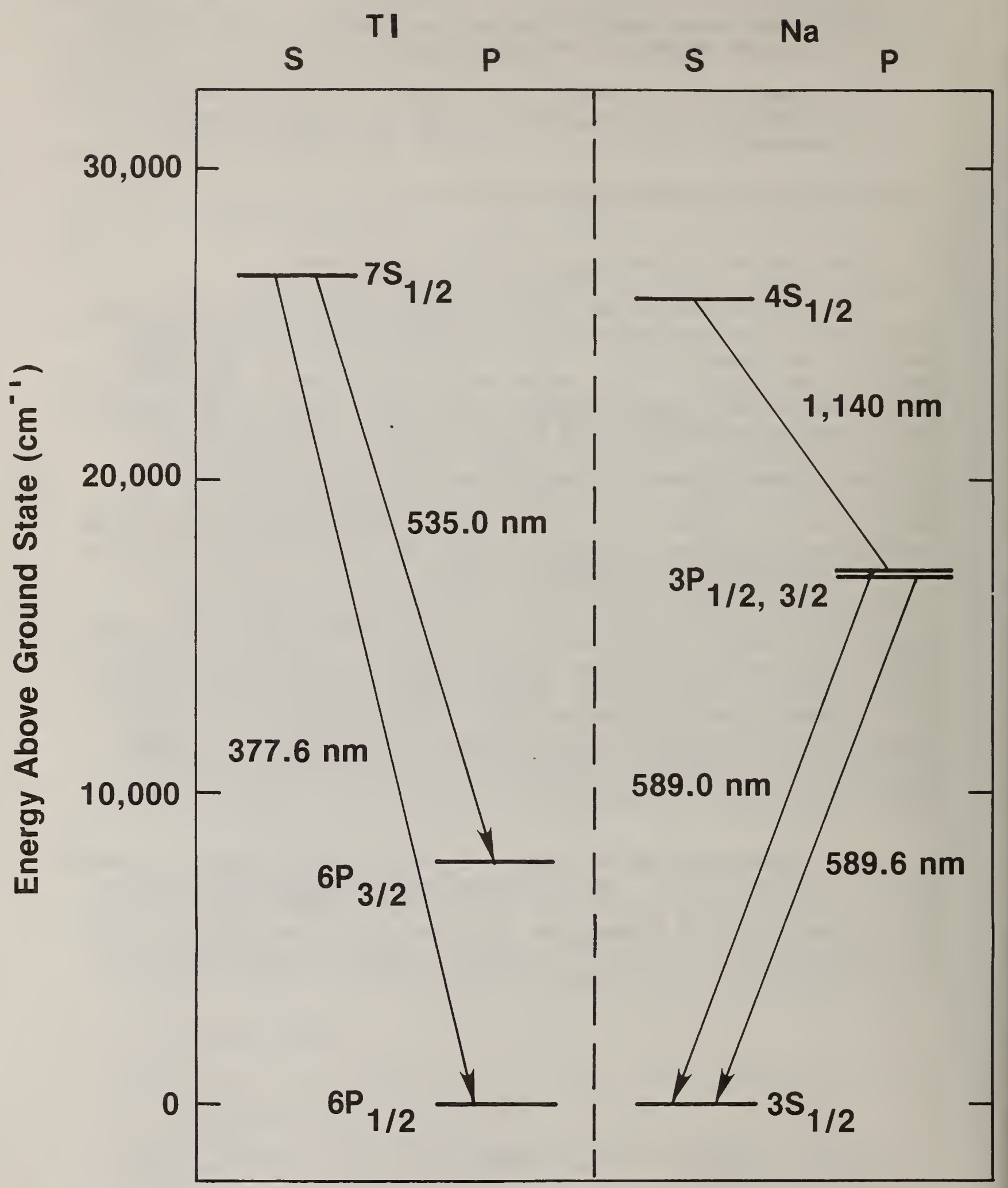

Figure 4. Partial energy level diagram for $\mathrm{TlI}$ and $\mathrm{NaI}$ lasers, showing origination of atomic laser lines. 
Table 1. Operating Characteristics and Analytical Results for TlI and $\mathrm{NaI}$ Lasers.

\begin{tabular}{|c|c|c|}
\hline & $\mathrm{T} 1 \mathrm{I}$ & $\mathrm{NaI}$ \\
\hline Resonance Wavelength(s), $\mathrm{nm}$ & 377.6 & $589.0,589.6$ \\
\hline Energy/Pulse, $\mu \mathrm{J}$ & 9 & 0.1 \\
\hline Nonresonance Wavelength(s), nm & 535.0 & $1140.3,1138.2$ \\
\hline Energy/Pulse, $\mu \mathrm{J}$ & 30 & 5 \\
\hline Operating Temperature, ${ }^{\circ} \mathrm{C}$ & 380 & 660 \\
\hline El ement Analyzed & $\mathrm{T1}$ & $\mathrm{Na}$ \\
\hline Limit of Detection, $\mathrm{ng} / \mathrm{mL}$ & 3 & 0.3 \\
\hline $\begin{array}{l}\text { Linear Dynamic Range, Orders of } \\
\text { Magnitude }\end{array}$ & $\sim 4$ & $\varsigma_{4}$ \\
\hline
\end{tabular}

A unique aspect of the NaI laser for LEI is the presence of the $1,140 \mathrm{~nm}$ nonresonance line whose lower level is the same as the upper level of the resonance line. This situation results in a two-photon stepwise excitation of $\mathrm{Na}$ atoms, a great advantage for LEI since it allows population of an excited state close to the ionization limit. In this case the stepwise excitation resulted in a 13-fold sensitivity enhancement over that of single photon excitation. The ultimate analytical impact of the atomic resonance line photodissociation laser will depend on the number of el ements for which such lasers are eventually constructed. In the meantime, the lasers demonstrated here, as well as other available lasers, may well be the source of choice for many specific, dedicated applications.

\section{References}

[1] Ehrlich, D. J., Maya, J., Osgood, R. M., Appl. Phys. Lett. $\underline{3}$, 931-933 (1978).

[2] Ehrlich, D. J., Osgood, R. M., Appl. Phys. Lett. 34, 655-658 (1979).

[3] Deutsch, T. F., Ehrlich, D. J., Osgood, R. M., Jr., Opt. Lett. 4, 378-380 (1979).

J. C. Travis, G. C. Turk 


\section{c. Reduction of Ionization Interferences in Laser Enhanced Ionization}

\section{Spectrometry}

Laser enhanced ionization (LEI) spectrometry in flames is based on the electrical detection of the thermal ionization of excited state atoms or molecules produced by laser excitation. For many elements a very high level of sensitivity is possible, but general application to the analys is of complex samples has been hampered by the method's susceptibility to ionization interference. This interference results from the unassisted thermal ionization of low ionization potential elements in the sample matrix. Recent advances in our understanding of how the LEI signal is collected have resulted in a better understanding of why this interference occurs, and have led to an experimental modification which has greatly reduced LEI susceptibility to ionization interference.

Electrical detection of LEI requires that an electric field be applied to the region of the flame being irradiated by the laser. Because of the vastly different mobilities of electrons and positive ions, this field is in most cases not uniformly distributed between the anode and cathode, but rather is concentrated near the cathode in a region known as the cathode fall or as the cation sheath. This sheath becomes larger as higher voltages are applied, but compresses as higher ion concentrations are encountered, as when significant matrix ionization is occuring. Most LEI work to date has utilized a split cathode configuration, where the burner head is used as the anode and a pair of metal plates just outside opposite sides of the flame form the cathode. This configuration has the advantage of leaving the flame physically unperturbed. However, in the presence of high ion concentrations resulting from matrix ionization, the cation sheath can collapse to within a very short distance from the cathode plates. When this occurs no electric field exists inside the flame near the laser beam, and consequently no LEI signal is collected.

A new electrode configuration has been developed which greatly reduces LEI susceptibility to ionization interferences. A water cooled cathode, constructed from 1/4" diameter stainless steel tubing is placed directly into the flame, one $\mathrm{cm}$ above the burner head anode. This allows the laser beam to be positioned very close to the cathode surface, thus reducing the possiblity of the beam being outside of the sheath. Water cooling prevents deterioration of the cathode. No contamination or memory effects have been observed.

Figure 5 shows the effect of varying the sodium concentration (matrix effects) on an LEI signal observed for $50 \mu \mathrm{g} / \mathrm{mL}$ of $\mathrm{Fe}$ for both the external split cathode plate configuration and the new internal watercooled cathode. A $1500 \mathrm{~V}$ potential is applied in both cases. The new configuration has improved the LEI tolerance from $\sim 100 \mu \mathrm{g} / \mathrm{mL}$ to over $3,000 \mu \mathrm{g} / \mathrm{mL}$ to the sodium matrix effect. The maximum allowable concentration of sodium ions in the aspirated solution is limited by arcing, which 


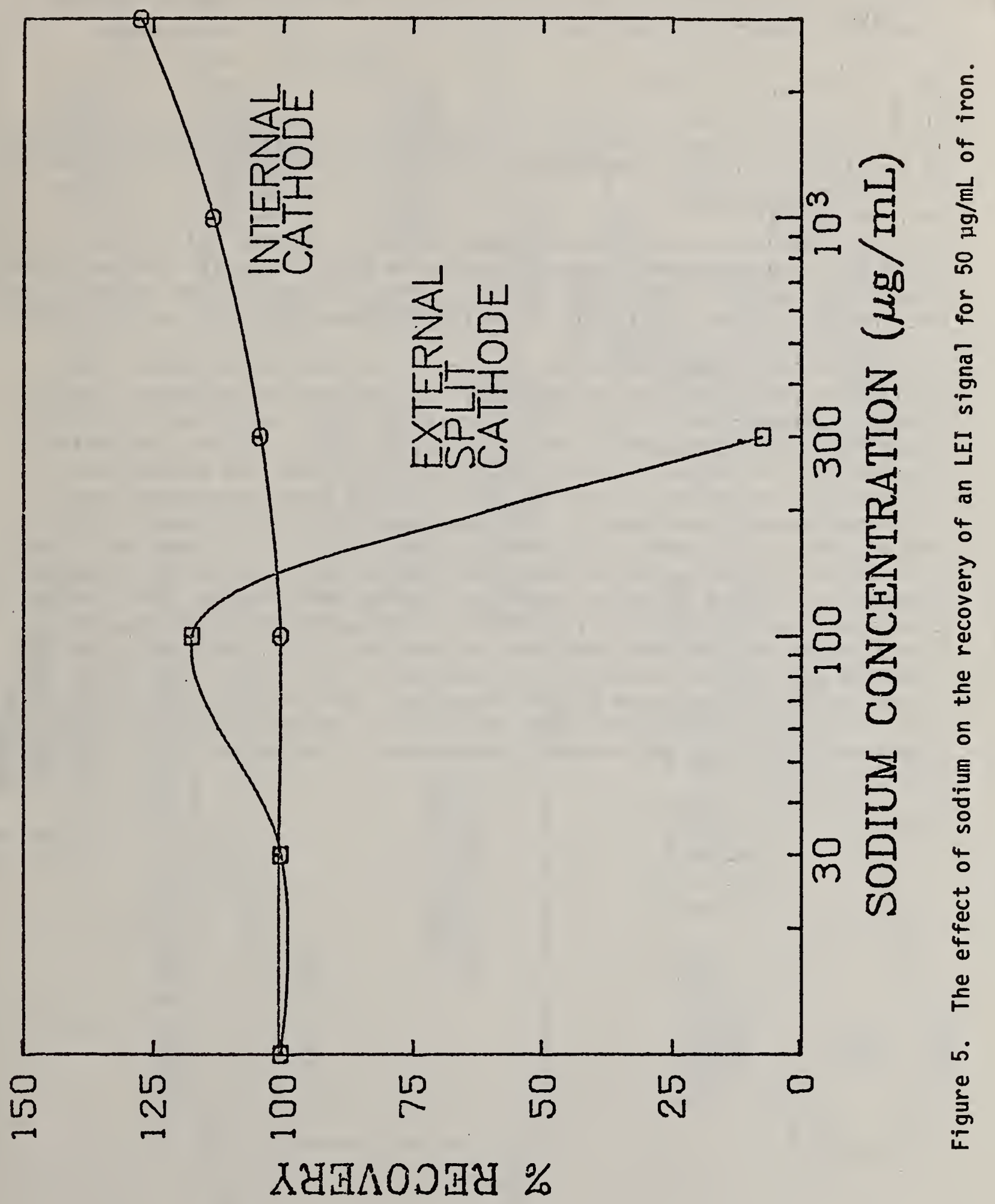


occurs at $\sim 5000 \mu \mathrm{g} / \mathrm{mL}$. This is a major improvement to LEI spectrometry allowing a more accurate analys is of a wide variety of complex samples containing easily ionizable materials.

G. C. Turk, J. C. Travis

d. Detection Limits of Some Trace Molecules of the Atmosphere by IR Diode Laser Absorption

All molecules except homonuclear diatomics have an infrared spectrum. This fact, in conjunction with the newly developed tunable infrared diode laser sources that have high spectral purity and power, make possible the detection of trace species by IR radiation absorption. We have set up a tunable diode laser system that incorporates a diode laser with some external optics and additional electronics as shown in figure 6 . This system allows the use of second derivative modulation techniques with a long-pass White cell. In this way, very low (ppt) detection limits were obtained for numerous molecules as shown in Table 2. First and second derivative techniques were used to discriminate from the baseline. Improvement in the net signal was at least $10^{2}$ over conventional absorption measurement techniques. The signal-to-noise of the weakest line corresponds to an absorption coefficient of $5 \times 10^{-8} \mathrm{~m}^{-1}$ for a $40 \mathrm{~m}$ cell. The total cell volume is 8 liters which means that the amount of sample needed for $1000 \mathrm{~Pa}$ pressure (normal operating pressure) is 5 millimoles. The technique is not only very sensitive but quite selective at the pressures used. Further increases in sensitivity are anticipated in the near future by using more stable diodes which will enable the use of longer time constants and signal averaging. This effort is the forerunner to a project in cooperation with the staff of the Gas and Particulate Science Division on the accurate measurement of molecular species in the atmos phere. 


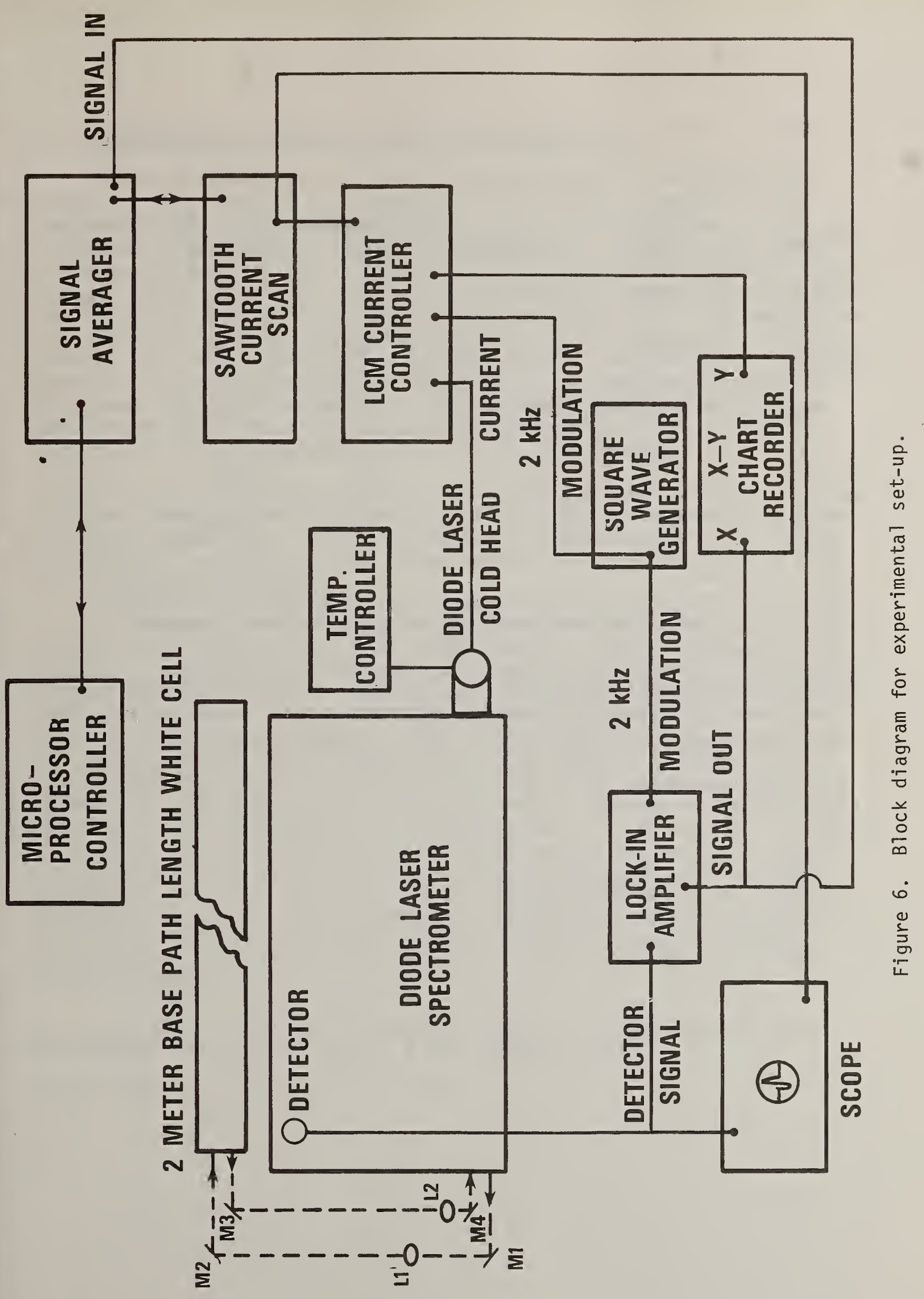


Table 2. Detection Limits of Trace Constituents of the Atmosphere.

\begin{tabular}{|c|c|c|c|c|c|}
\hline Molecule & Band & Transition & $\begin{array}{l}\text { Pathlength } \\
(\mathrm{m})\end{array}$ & 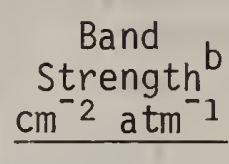 & $\begin{array}{l}\text { Detection } \\
\text { Limit }\end{array}$ \\
\hline $\mathrm{N}_{2} \mathrm{O}$ & $00^{\circ} 1-00^{\circ} 0$ & $R(8)$ & 24 & 1173 & 200 \\
\hline $\mathrm{NO}_{2}$ & $00^{\circ} 1-00^{\circ} 0$ & $16_{1,16-17_{1,17}}$ & 40 & 2000 & 100 \\
\hline $\mathrm{CS}_{2}$ & $11^{1} 1-01^{1} 0^{a}$ & $P(27)$ & 24 & 2220 & 40 \\
\hline $\mathrm{CO}_{2}$ & $04^{\circ} 1-04^{\circ} 0^{a, d}$ & $P(23)$ & 40 & 2440 & 20 \\
\hline OCS & $04^{\circ} 0-0^{\circ} 0^{\mathrm{a}}$ & $P(15)$ & 24 & 2400 & 20 \\
\hline
\end{tabular}

${ }^{\mathrm{a}}$ The actual measured transitions were from these bands. The detection limits in column 6 were estimated using relative band strengths. b Strongest band.

${ }^{C}$ Extrapolated detection limit (i.e., signal is twice the standard deviation of the noise) in ppt for strongest band.

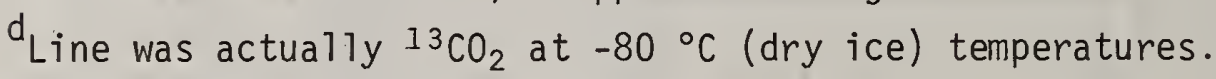

R. L. Sams, J. R. DeVoe 


\section{e. Trace Organic Analysis by Multiphoton Ionization}

Resonance-enhanced multiphoton ionization (MPI) is an especially promising technique for the detection of trace organic species in the vapor phase using high-powered, tunable lasers. The technique, which was developed at New York University (Stoneybrook), utilizes a laser beam which passes into the gas of a proportional counter or ion chamber. The photoionization process is detected by measuring the current produced from the detector which is in coincidence with the laser pulse. The method has potential for very low detection limits with high selectivity. Unfortunately, with large organic molecules, the optical selectivity is inherently poor due to the spectral density resulting in line overlap. Because of this, our initial investigations of the technique have assumed the need for further selectivity by such means as multiphoton laser excitation coupled with gas chromatography and/or mass spectrometry.

Experiments with chlorobenzene and napthalene have shown the expected spectral character, and sensitivity at the $10 \mathrm{ppb}$ level in air, using our flashlamp-pumped laser. The MPI spectrum of chlorobenzene at $\sim 2 \mathrm{ppm}$ in air is shown in figure 7 . The intense band head at $\sim 37050 \mathrm{~cm}^{-1}$ is used for analytical measurements.

An especially revealing series of experiments utilized a gas chromatograph with a hybrid MPI/el ectron capture detector (ECD), as shown in figure 8 . Representative chromatograms are shown in figure 9 . In the top series, (a) through (c), the ECD is operated normally, showing two solvent peaks followed by a chlorobenzene peak. When partial (b) and full (c) laser power is applied, the ECD response to chlorobenzene is enhanced over the unperturbed (a) case. The origin of this behavior is not obvious, but the possibility of photofragmentation should be considered. When the normal ECD sensitivity is drastically reduced by the use of a reverse bias (series $d$ - f) laser-produced ionization for partial (c) and full ( $f$ ) laser irradiation may be seen as a negative-going hash. The ionization signal is negative because ECD response is electron absorption, and the signal is ragged because the laser is pulsed. The perturbation to the chlorobenzene peak is taken to correspond to resonance-enhanced MPI (figure 7). The perturbation at the trailing edge of the solvent peak is a puzzle, but could represent non-resonant photo-detachment of an electron from a negative solvent ion.

We plan to investigate these observations in future studies using mass spectrometric detection and a more suitable laser.

R. K. Simon, J. R. DeVoe, J. C. Travis 


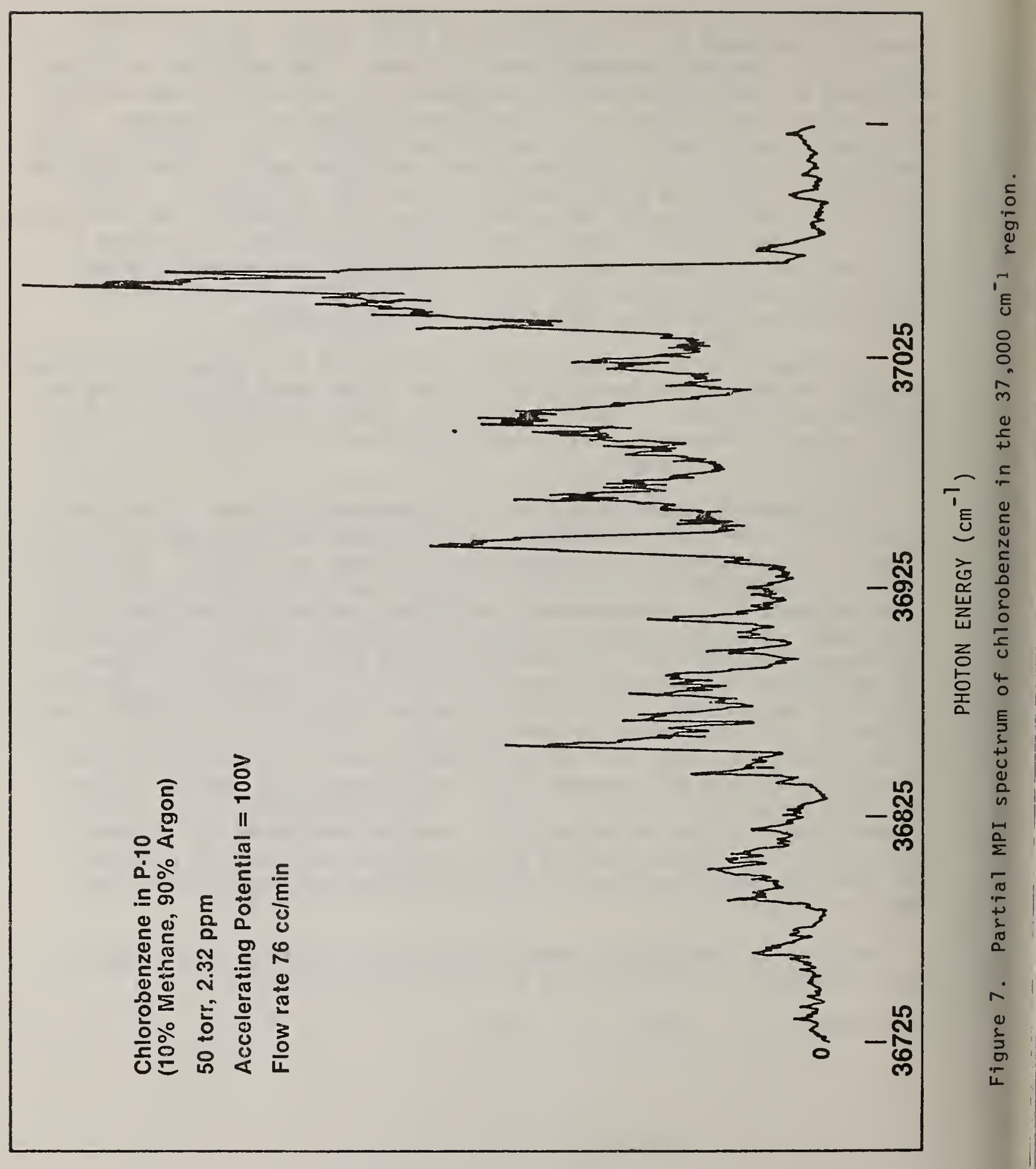

7甘NIIS IdW $\exists \Lambda I \perp \forall 7 \exists Y$ 


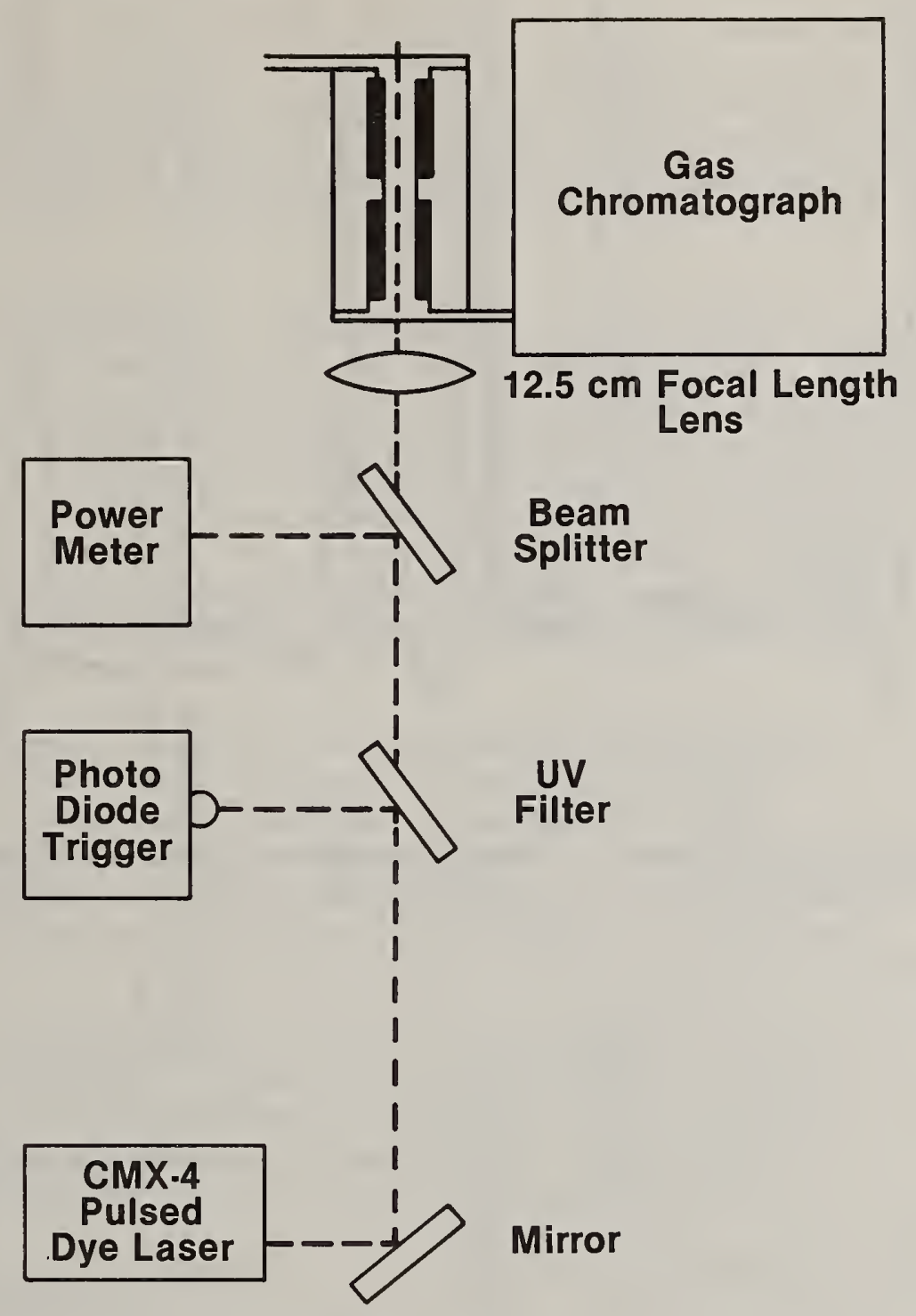

Figure 8. Experimental arrangement for GC detection with a laser-irradiated electron capture detector. 


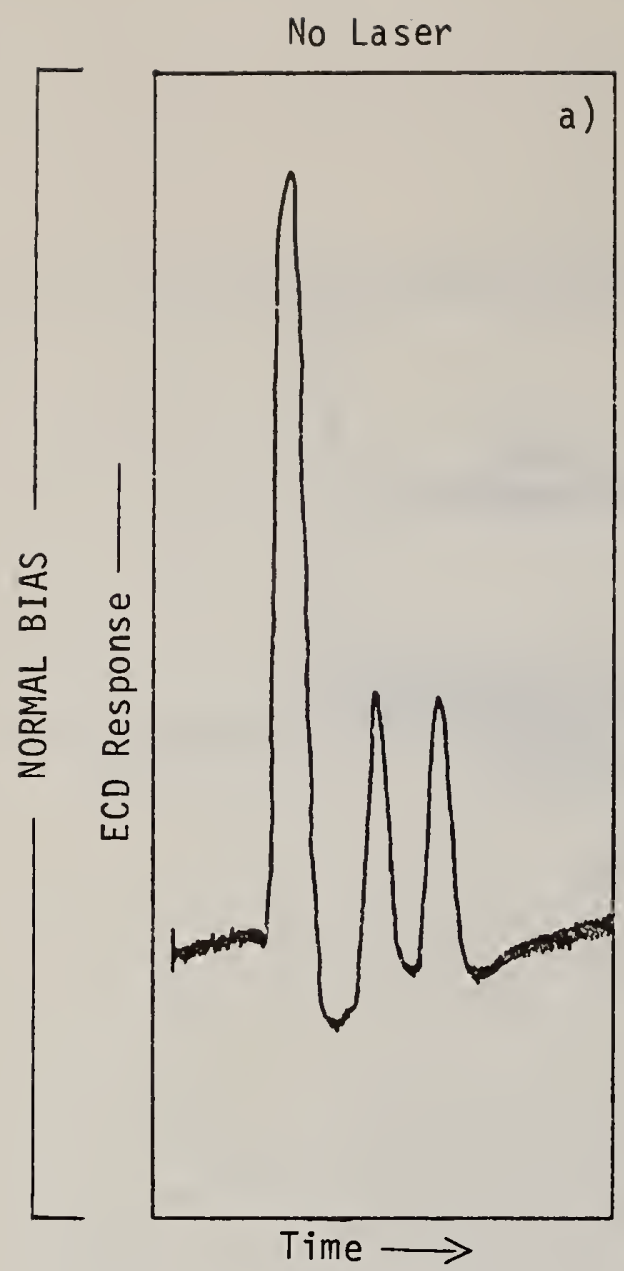

Partial Laser Power

\section{Full Laser Power}
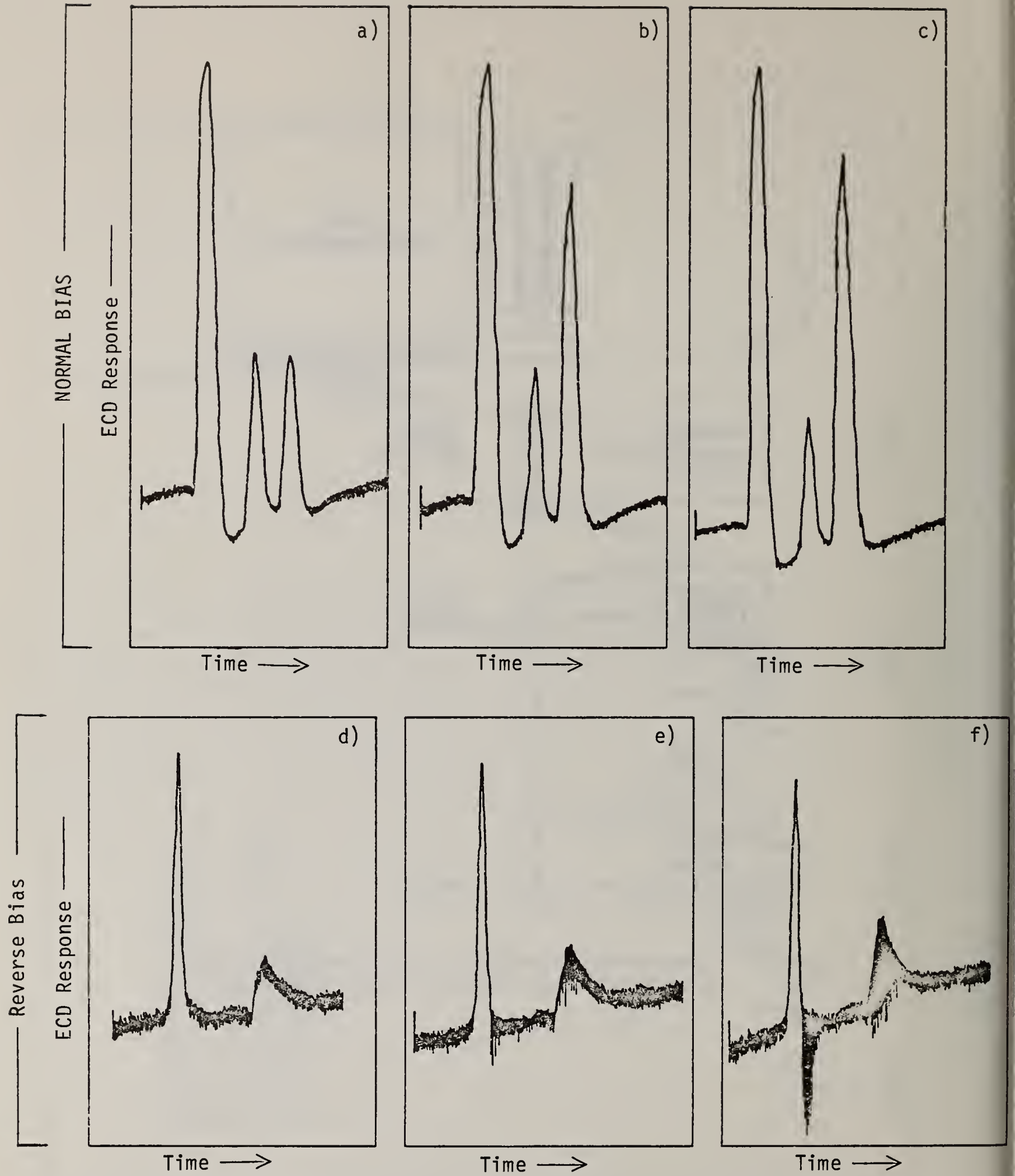

Figure 9. Gas chromatograms showing solvent and chlorobenzene peaks with the ECD normally biased (top) and reversed-biased (bottom). Effects due to the laser in the chlorobenzene (third) peak are seen by comparing laser off (1eft) to laser on (center $=$ $50 \%$ attenuated; right $=$ ful1 laser intensity) chromatograms . The laser effect on the solvent peak (bottom right) is taken to represent wavelength-independent el ectron photo-detachment. 


\section{f. Instrumentation Project}

The people in this project interface with all Center personnel to provide technical support in the areas of computers and instrumentation. The work performed can be classified into three major areas: 1) microcomputers (microprocessors) for data acquisition, manipulation, and instrument control; 2) instrument design and modification; and 3) instrument-computer repair. Selected examples of the work in each category are:

1) Microcomputers - development of a microcomputer based system consisting of one 16 bit microcomputer, 1 Mbyte of memory, parallel-serial I/0, terminal, CRT, dual floppy discs with BASIC and FORTRAN costing $\$ 7 \mathrm{~K}$ that has an overall performance approximately equivalent to a PDP 11-34 minicomputer-adaptation or updating of microcomputers for use on ESCA-Auger spectrometers, IR diode laser system, gas handling systems, and low level ${ }^{14} \mathrm{C}$ radiation counters to provide foregroundbackground capabilities;

2) Instrument design, construction and modification construct triple filament controller for mass spectrometry, nuclear waste container scanner incorporating a unique stepping motor drive designed to minimize vibration, an optoacoustic detector, and a potentiostat for fast electrochemical reactions; and

3) Instrument-computer repair - in addition to the usual types of instrument repair, the increasing use of microcomputer systems results in the need to replace defective boards and floppy discs, upgrade software, and develop special software.

R. W. Shideler, F. C. Ruegg, J. K. Langland, B. McDiffett, S. Mund, A. Beals, L. Bergen 
3. Outputs and Interactions

a. Publications

Turk, G. C., Mallard, W. G., Schenck, P. K., and Smyth, K. C., "Improved Sensitivity for Laser Enhanced Ionization Spectrometry in Flames via Multiple Wavelength Stepwise Exitation", Anal. Chem. 51, 2408-10, (1979).

Turk, G. C., Travis, J. C., DeVoe, J. R., and O'Haver, T. C., "Laser Enhanced Ionization Spectrometry in Analytical Flames", Anal. Chem. 51, 1890-90 (1979).

Travis, J. C. and DeVoe, J. R., "Optogalvanic Spectroscopy", scheduled for publication in Lasers and Chemical Analysis, Hieftje, G. M., Lytle, F. E., and Travis, J. C., eds., Humana Press, Clifton, N. J. to be published.

Travis, J. C., editor with Lytle, F. E., and Hieftje, G. M., "Laser and Chemical Analysis", Human Press, Clifton, N. J. to be published. Proceedings of the 1979 Analytical Chemistry Summer Symposium.

Ehrlich, D. J., Osgood, R. M., Jr., Turk, G. C., Travis, J. C., "Atomic Resonance-Line Lasers for Atomic Spectrometry", Anal. Chem. $\underline{52}, 1354-5$ (1980).

Green, R. B., Keller, R. A., Luther, G. G., Schenck, P. K., Travis, J. C., United States Patent No. 4,184,127, Jan. 15, 1980 entitled "Frequency Stabilized Laser".

Scheel ine, A., Travis, J. C., DeVoe, J. R., Watters, J. P., "Discovery of an Electrical Post-Pulse in the Surroundings of a High Voltage Spark Discharge" Accepted for publication Spectrochimica Acta B.

Scheeline, A., Norris, J. A., Travis, J. C., DeVoe, J. R., Walters, T. P., "Particulates Formed by a Stabilized High Voltage Spark Discharge", accepted for publication Spectrochemica Acta B.

Maki, A. G., 01 son, W. B., Sams, R. L., "High Resolution Infrared Spectrum of the 859 and $1711-\mathrm{cm}^{-1}$ Bands of Carbonyl Sulfide (OCS)", $\underline{J}$. Mol. Spectros. 81 (1980).

Sams, R. L., 01 son, W. B., "Analysis of the High Resolution Infrared Spectrum of the $V_{2}$ Bending Mode of $\mathrm{HOCl}$ at $1238 \mathrm{~cm}^{-11}, \underline{\mathrm{J}}$. Mol. Spectrae accepted for pubtication.

Seger, P. M., and Sams, R. L., "Diode Laser Spectra of the $\mathrm{V}_{2}$ Band of $\mathrm{H}_{2}{ }^{12} \mathrm{CO}$ and $\mathrm{H}_{2}{ }^{13} \mathrm{CO}^{\prime \prime}, \underline{\mathrm{J}}$. Mol. Spectrae. Accepted for publication. 
b. Talks

J. R. DeVoe, "Laser Enhanced Ionization in Flames", Eastern Analytical Symposium, New York, N. Y. Oct. 1979 (invited).

R. L. Sams, J. R. DeVoe, "Measurement of the $\mathrm{V}_{3}$ Band of ${ }^{14} \mathrm{CO}_{2}$ by Tunable Diode Laser", Thirty-fifth Symposium on Molecular Spectroscopy, Ohio State University, Columbus, Ohio, June, 1980.

R. Simon, "Multi-photon Ionization of Organic Compounds" Seminar, Chemistry Department, Kansas State University, July 9/25, 1980.

c. Invited Talks by J. C. Travis

(with G. C. Turk, S. J. Weeks, and J. R. DeVoe) "Trace Metal Analys is by Laser Enhanced Ionization in Flames", Expochem 179; Houston, Texas; Oct. 22, 1979.

"Laser Enhanced Ionization in Flames", Chemistry Dept. Colloquium; Villanova University; Philadelphia, PA.; Nov. 27, 1979.

"Perspectives on The Use of Lasers for Chemical Analysis", New York Academy of Sciences; New York, N. Y.; Dec. 13, 1979.

"Perspectives on The Use of Lasers for Chemical Analysis", Center for Analytical Chemistry Colloquium; National Bureau of Standards; Washington, D. C.; Dec. 19, 1979.

"Laser Opto-galvanic Spectroscopy for Trace Metal Detection", Society for Applied Spectroscopy, Baltimore-Washington Section; Rockville, Maryland; Feb. $19,1980$.

"Laser Enhanced Ionization in Flames for Trace Metal Analysis", Chemistry Department Seminar, University of Florida; Gainesville, FL; Oct. 22, 1980.

(with Richard K. Simon and James R. DeVoe) "Selective Laser Ionization for Atomic and Molecular Trace Analysis", Dept. of Energy "Workshop on Advanced Laser Technology for Pollutant Measurements; Harper's Ferry, W. Va.; June 10, 1980.

(with G. C. Turk and P. K. Schenck), "Progress in Laser Enhanced Ionization", Federation of Analytical Chemistry and Spectroscopy Societies (FACSS-VII); Philadelphia, PA.; Sept. 30, 1980.

d. G. C. Turk Talks, Sept. 1979 - Sept. 1980

"Laser Enhanced Ionization Spectrometry", G. C. Turk, University of Maryland, Analytical Chemistry Division Seminar, College Park, Md., Oct. 1979.

"Reduction of Matrix Interferences in Laser Enhanced Ionization Spectrometry", G. C. Turk, J. C. Travis, and J. R. DeVoe, 7 th Annual FACSS Meeting, Philadelphia, Pa., Sept. 1980. 
"Atomic Resonance-Line Lasers: New Sources for Analytical Atomic Spectrometry", P. J. Ehrlich, R. M. Osgood, Jr., G. C. Turk, and J. C. Travis, 7 th Annual FACSS Meeting, Philadelphia, Pa. Dept. 1980. (Keynote Talk).

e. Committee Assignments

James R. DeVoe

CAC Committee on Computer Requirement

NBS Committee on Selection of Microprocessor for the Storeroom

NBS Computer Evaluation Committee

F. C. Ruegg, Jr.

NBS Committee on Selection of Microprocessors for the Storeroom

R. W. Shideler

NBS Committee for Electronic Storeroom

J. K. Langland

NBS Committee for Electronics Storeroom

NBS Committee on Selection of Microprocessors for the Storeroom 
II. Inorganic Analytical Research Division

Ernest L. Garner, Chief

Thomas J. Murphy, Deputy Chief

A. Division Overview

The diverse analytical competences in the Inorganic Analytical Research Division are organized functionally into the four broad areas of activation analysis, atomic and molecular spectrometry, analytical mass spectrometry, and electroanalytical chemistry. This structure provides both the flexibility and the analytical capability to address a wide variety of research problems and also to analyze the full range of materials utilized in the various research activities at NBS, other Federal agencies and, when requested and appropriate, other institutions, either private or public. Within this framework, the research and measurement priorities of the Division are established on the basis of potential public benefit and of developing the science to accurately determine the concentration of inorganic species in the numerous materials problems which confront government and industry.

During the past year, the Division witnessed a continuation of the growth and proliferation of demands for inorganic analytical chemistry. These requests reflect a broad spectrum of needs ranging from the users of calibration or unique service analyses to the agencies/organizations primarily concerned with analytical chemistry as a tool for the solution of problems of national and/or international impact. With ever increasing frequency, this constituency is seeking accurate multi-element data for complex matrices such as drilling mud, sludges, retort water, high performance metals, oil shale, and effluents or leachates from a variety of manufacturing and process streams. The reality of our current position with respect to the need for more accurate multi-element data is reflected by the lack of a single technique, either free of matrix interferences or with the dynamic calibration range, to accurately measure 10 or more trace elements in a complex matrix. As such, a major thrust in the Division's long term research is the ultimate development of basic calibration methodology which will yield accurate multi-element measurements for trace elements under a variety of matrix and concentration conditions.

With multi-element analysis as one of several pressure points, the past year's activities reflect a predominant orientation to analytical measurements with a significant effort devoted to developing new or improved methods. For example, research to more accurately characterize Standard Reference Materials included the development of D.C. plasma spectrometry for trace element analysis, and reference methods for bismuth, iodine, aluminum, and carbon in material types which range from biological to sophisticated high performance metals. In addition to this research, a major effort, approximately 25 percent of the total, was devoted to analytical measurements to certify SRM's. 
Other applied research and measurement, in support of major NBS activities or in direct support of other government agencies, provided assistance to the Recycled 0 il Program, Environmental Measurements, Nuclear Safeguards, Resource Recovery, the National Environmental Specimen Bank, Trace Element Profiles for the Chesapeake Bay, and Trace Elements in Biologics and Injectibles. As an example, the activities with respect to recycled oil provide a diversified mix of research to develop an operational ICP system for high sensitivity multi-element analysis of selected elements in oil, methods evaluation and speciation studies by atomic absorption, analytical measurements and speciation studies by activation analysis, investigations to evaluate ion chromatography as a quantitative method for chlorine and bromine, and also an assortment of service analyses.

Fossil fuel waste sampling and characterization, acid rain, and trace metals in different injectable types of biological materials represent new research and measurement activities. An ultimate goal in each of these areas is the production and accurate characterization of trace elements in quality assurance materials or Standard Reference Materials. For fossil fuel waste residues, a multi-year effort is oriented to developing the analytical methods, leach protocols, and quality assurance materials to accurately characterize both the leachate and waste residues of the highly variable waste streams of conventional and emerging technology fossil fuel power plants. As a part of this research, a rotary extractor for leach studies has been constructed and preliminary testing completed. This unit as well as improved leach protocols and a synthetic leachate represent significant progress in establishing an accurate data base for waste residues such as coal fly ash, coal bottom ash, sludges, and other effluents from the residues of power producing plants.

In high accuracy measurements, a redetermination of the atomic weight of silver was completed. The overall accuracy of this is expected to be approximately 1 in $10^{6}$. At this significantly improved accuracy, the uncertainty in the atomic weight of silver is no longer a major factor in the calculation of a high accuracy value of the Faraday constant from the electrochemical equivalent of silver. The NBS atomic weight research represents one of the few remaining sources of new atomic weight data and is one of the prime reasons for continued improvement in the precision of isotopic ratio measurements. This improvement is demonstrated by recent isotopic measurements with precisions equal to or greater than those obtained in the gravimetric preparation of synthetic calibration mixtures.

For next year, planned activities will focus on automation and research to increase sensitivity, accuracy, and multi-element capability for trace elements. One of the more urgent automation needs is the design and construction of rotators, sample changers, and manipulators for automated irradiation and counting of samples by activation analysis. In this connection, a new computerized system for instrument control, data acquisition, computation, and statistical analysis of data will also be required to handle the increased volume of samples which is anticipated over the next several years. Recent advances in microprocessor technology will definitely allow improved control of mass spectrometers and will, 
over a multi-year period, lead to fully automated instruments for both high precision and high accuracy measurements. The third area of immediate concern is the whole process of data acquisition, correlation, curve fitting, interelement corrections, and background corrections leading to improved calibration in emission and atomic absorption spectrometry.

Compositional depth profiling and mass spectrometry are target areas for increased research. The intent of depth profiling is to develop a facility at NBS for depth location of selected elements using thermal neutron induced reactions. The ultimate goal of this research is to demonstrate the limits of resolution and sensitivity and to extend the technique to isotopes other than ${ }^{3} \mathrm{He},{ }^{6} \mathrm{Li}$, and ${ }^{10} \mathrm{~B}$. Research in mass spectrometry will focus on the use of lasers and other techniques to better understand some of the processes which will improve the sensitivity and accuracy of isotopic measurements.

Calibration methods which are applicable over a large dynamic range and for a large number of different matrices are keys to improving the quality of multi-element data. As such, the multi-element problem will be attacked on several fronts with immediate goals of improving the calibration methods for the ICP and as a means of making emission spectrometry more quantitative. Closely related to this effort is the continuing effort to utilize wavelength modulation in conjunction with a high intensity continuum source and an echelle monochromator to reduce the effects of interference in atomic absorption measurements. This basic approach has significant potential for multi-element analysis. Finally, epithermal activation analysis techniques in conjunction with an antiCompton shield will be investigated as a means of improving the quality of multi-element measurements. Ultimately, several of the activation techniques must be explored as a means of quickly and accurately establishing the concentration levels of a large number of elements.

Two new areas which are expected to become targets of opportunity for long-term research are measurements and standards for nuclear waste management and metal speciation studies. Nuclear waste disposal is a major national problem requiring a coordinated effort from various units of the Division to produce accurate trace and ultratrace element measurements of glasses, basalts, and other potential storage materials for long-lived radioactive waste. For speciation, the need to know not only the accurate concentration of trace elements but also the chemical species will require a multifaceted approach. The ultimate development of this capability will have a profound impact in areas such as energy production, environment, industrial processes, food, nutrition, and health.

\section{Activation Analys is Group: Overview}

Research in activation analysis at NBS is a continuing effort to improve analytical accuracy, sensitivity and selectivity in diverse matrices of technological and scientific importance. Improved measurements in activation analysis can be realized both by systematic evaluation and elimination of errors associated with current methods, 
and by the development of new and improved techniques. Both approaches have been used to improve the measurement capability in trace analysis. Innovation within the group and work at the frontier of the field has earned the respect of the trace analytical community. This group is looked to for leadership in nuclear methods for accurate trace analysis. The group members continue to develop specific competences in the areas of reactor neutron activation analys is (NAA), photon activation analysis (PAA), nuclear track technique (NTT), and prompt gamma activation analys is (PGAA).

The Activation Analys is Group is conducting research and analys is in four basic areas. Basic research is aimed towards the improvement of the analytical measurement system using nuclear techniques. The end result of this activity is to have a constantly expanding analytical capability which can be applied to all areas of elemental analysis. Applying analytical research to specific programs, the group utilizes its capability to provide reliable analytical data for the study of systems dependent on trace elemental composition. Analyses performed on new Standard Reference Materials are an extremely important area of the group's activity. Special emphasis is placed on the accuracy of analytical results. A small but important part of the group's analytical program is "service analysis." This is chiefly undertaken to expedite other programs, by providing crucial analytical data, or a unique measurement when needed. The activities in the different program areas keep the group members aware of developments in other fields, and in many instances the "real world" samples obtained for analysis spur new research ideas.

Recent accomplishments in research are highlighted by the contributions to the establishment of a "definitive" or reference method for the determination of aluminum. Other continuing efforts on the selective analysis of specific elements have led to the development of two independent procedures for the determination of iodine in biological matrices, which will provide reliable results for iodine at the $\mu \mathrm{g} / \mathrm{g}$ level and below. A highly selective separation for bismuth from photon-activated nickel alloys makes the determination of this element feasible at the $\mathrm{ng} / \mathrm{g}$ level and below.

The group is substantially supporting major NBS programs with research and analysis. Specific efforts have been made in sampling and sample preparation methodology in order to provide meaningful analytical samples in connection with the National Environmental Specimen Bank (NESB). The extended instrumental capabilities help the Activation Analys is Group to provide the large quantity of reliable analytical data which, combined with the results of other analytical techniques, provide essential information on environmental and biological systems. This information is needed, for example, in the Chesapeake Bay Program and in the pilot NESB Program. Sampling and analysis techniques have been developed for the dependable measurement of key elements in both homogeneous and non-homogeneous oils in support of the ongoing Recycled $0 i 1$ Program. Studies in support of the development of low-enriched uranium oxide, for use as SRM's for non-destructive analysis are being made to identify all possible interferences to the nuclide of interest. 
The group participated in the certification of new biological metals, minerals, and environmental SRM's. Analytical problems solved by the group for other programs included the determination of chlorine in CdS-samples using epithermal reactor neutrons for activation. Selenium was determined in extremely small samples of mitochondria in collaboration with researchers at the NIH. The current state of high precision measurements enables the group to carry out determinations of activation cross sections (e.g. ${ }^{59} \mathrm{Co}$ to ${ }^{60 \mathrm{~m}} \mathrm{Co}$ and ${ }^{609} \mathrm{Co}$ to ${ }^{61} \mathrm{Co}$ ) and to contribute to the accuracy of physical constants.

Immediate future goals are the improvement of the instrumental analysis capabilities by advanced counting methodologies including coincidence and anti-coincidence spectrometry and the development of a precise monostandard technique. New radiochemical methodology will provide results on ultratrace elements and, in collaboration with separation chemists, the quantitative determination of organic species will be investigated. Efforts will be directed to the determination of the location of traces within a matrix.

Rolf L. Zeisler, Group Leader; B. Stephen Carpenter, Kathleen A. Fitzpatrick, Ronald F. Fleming, Thomas E. Gills, Robert R. Greenberg, Sally H. Harrison, James Koskoris, Richard M. Lindstrom, George J. Lutz, M. James Blackman

\section{Electroanalytical Chemistry Group: Overview}

The Electroanalytical Chemistry Group's expertise focuses on, but is not limited to, the utilization of electrochemical principles for chemical analysis. The techniques employed include voltammetry, coulometry, ion-chromatography, amperometry, potentiometry, and conductimetry.

A major effort of this group is concerned with the development and certification of Standard Reference Materials. This activity is of three types: research, acceptance testing, and analysis for certification. Two research projects were undertaken this year. One involved a development of an improved method for gravimetric determination of aluminum in bauxite. The other was the development of a method for the determination of trace amounts of iodide in biological and botanical matrices by differential pulse polarography. Three materials (benzoic acid, tris (hydroxymethyl)aminomethane, and potassium dichromate) were assayed coulometrically, prior to their acquisition in bulk, for use as renewal Standard Reference Materials. Two of the three materials were acceptable and will be certified next year. Trace metals were determined for certification of estuarine sediment, bovine liver, citrus leaves, line pipe steel, and alloy steels by voltammetry. Ion-chromatography was used to certify the sulfur content in several oils, and the chlorine and sulfur content in citrus leaves and bovine 1 iver.

A significant effort was expended in the improvement (particularly in extending the range) of the sodium alcoholate ion-chromatographic method for the determination of chlorine and bromine in recycled oils. 
As part of the National Environmental Specimen Bank Program, human liver samples were analyzed for trace metals by cathode ray polarography. A set of four oils, ranging in sulfur content from 0.5 percent to 4 percent, were analyzed by ion-chromatography as part of a cooperative program between the National Bureau of Metrology in France and the Office of Standard Reference Materials.

A multi-year project concerned with acid rainfall has been initiated. The scope of this project is to investigate and identify the problems and present limitations in the measurement of acidity and $\mathrm{pH}$ in rainwater. Based on the findings, research will be conducted to develop stable acid rain reference materials and to develop protocols for $\mathrm{pH}$, acidity, and conductivity measurements in low ionic strength, unbuffered solutions. This will require the strengthening of the group's competences in $\mathrm{pH}$, potentiometry, and conductimetry.

During the past year, a programmable computing integrator was added to the ion-chromatograph which greatly expands its usefulness as an analytical tool. It is hoped that similar data acquisition and data management devices can be acquired for voltammetry, coulometry, and potentiometry to improve measurement capabilities and to handle routine mathematical operations.

In the coming year, in addition to an anticipated intense sampleanalysis effort, the Electroanalytical Chemistry Group will engage in research to develop a species-selective and sensitive anion detector for ion-chromatography based on amperometric principles. Investigations will be conducted as to the sources of and remedy for possible systematic errors in high-precision coulometric measurements which may adversely affect the accuracy of the method. Continuing into the next year is a project concerned with the determination of aluminum and other metals at trace and ultra-trace levels in serums and injectibles.

William F. Koch, Group Leader; E. June Maientha1, George Marinenko, Jeffrey W. Stolz, Pan Xiurong,

\section{Analytical Mass Spectrometry Group: Overview}

The primary objectives of the Analytical Mass Spectrometry Group are the development and maintainence of a capability for high accuracy isotope ratio measurements. The increasing need for such high quality analytical measurements on sub-microgram amounts of material has required continual improvement in chemical separation and purification techniques and instrumentation. To accomplish these tasks, the mass spectrometry group is composed of essentially two sub-groups. The chemistry sub-group is responsible for all of the chemical aspects of the group's work. Expertise in separation technology, isotope equilibration, sample dissolution, purification, and "contamination-free" sample handling are primary concerns in this area. The other sub-group specializes in inorganic mass spectrometry. This sub-group has considerable expertise in high accuracy mass spectrometric isotope ratio measurements and is responsible for 
developing improved methodology and instrumentation for spark source, electron impact, thermal ionization, and surface ionization mass spectrometry.

A significant effort has been expended in the areas of improved analytical measurements and basic methodology. Major accomplishments in analytical measurements include nuclear standards, atomic weights, and methodology needed for environmental and health related problems. A $233 \mathrm{U}$ spike solution (SRM 995) has been certified for assay and isotopic composition by comparison to a uranium metal standard (SRM 960) and a ${ }^{235} \mathrm{U}$ spike solution (SRM 993). The intercomparison of the three uranium SRM's showed internal consistency of better than 0.1 percent. Analytical work has been completed for the determination of the isotopic composition and assay of a $244 \mathrm{Pu}$ SRM. These results, together with those from Los Alamos Scientific Laboratory, will be used in the certification of this new material. Concurrent research on the analysis of nanogram quantities of $U$ and Pu loaded on single ion exchange resin beads has produced precisions of better than 0.1 percent for isotope ratio measurements.

Research and measurements for a redetermination of the absolute isotopic abundance and atomic weight of silver have been completed. Preliminary statistical evaluation of the data indicates a total uncertainty for the atomic weight of less than $1 \mathrm{ppm}$, a factor of 100 improvement over the currently accepted value. A more accurate value for the atomic weight of silver is needed because of a recent high accuracy redetermination of the electrochemical equivalent of silver. The uncertainty in the atomic weight of silver thus became the largest component of uncertainty in the value of the Faraday constant.

Sulfur concentrations have been certified in 12 iron base SRM's by isotope dilution-spark source mass spectrometry using a sealed tube dissolution and equilibration method. Sulfur blanks have been reduced to less than $1 \mu \mathrm{g}$ so it is now possible to analyze samples containing less than 10 ppm sulfur.

In a joint program with the Department of Nutrition and Food Science, Massachusetts Institute of Technology, a study was undertaken to determine whether or not oral administration of the stable isotope $70 \mathrm{Zn}$, at physiologically relevant concentrations, would allow the measurement of zinc enrichment with sufficient accuracy to investigate zinc metabolic kinetics in human plasma. Measurements on blood samples at MIT by neutron activation analysis and at NBS by mass spectrometry show this approach to studying metabolic processes to be feasible.

Major accomplishments in the area of improved methodology include development of mass spectrometric procedures for the analysis of elements not previously measured by this group, and improvements in instrumentation to increase precision as well as analytical output. Techniques have been developed for the isotopic analysis of microgram quantities of vanadium and iodine with precisions approaching 0.1 percent. A solid state filament control (which controls the current supplied to the mass spectrometer sample filaments with a stability of better than 0.01 percent) has been 
developed and tested. This unit has considerably improved the intraanalys is precision.

Future plans call for detailed studies of the mechanisms involved during the sample mounting and vaporization/ionization processes to allow better control of existing mass spectrometric procedures and to aid in the development of new procedures. This will include studies, by surface techniques, of the chemical and crystallographic species formed on the filament, and identification of the positive, negative, and neutral species formed during the ionization process. The successful analys is of iodine by negative ionization techniques will be followed by efforts to develop mass spectrometric procedures for other elements which are potentially ionizable as negative ions.

The use of lasers as an alternative ionization mode for spark source mass spectrometry will be investigated. The peak power available from lasers has become high enough for the laser driven plasma to be an efficient means of ionization. The energy that is available in these plasmas far exceeds that which is required to ionize all elements. This should result in both high absolute sensitivities and smaller differences in sensitivity between elements.

Future efforts in instrumentation are aimed at the development of fully automated mass spectrometers with accuracies and precisions equal to or better than can be obtained by manual operation. The general approach is a systematic upgrading of the major electronic sub-assemblies associated with the mass spectrometer. This will require research in specific areas to develop prototypes which can eventually be assembled as a fully automated instrument. Instrumental improvements scheduled for the near future include replacement of existing optical pyrometers used for setting filament temperatures with an infrared detection system, which under computer control will automatically reproduce and mainta in constant filament temperatures, and more extensive automation of magnetic field selection, baseline measurements, and ion beam focusing.

We also plan to automate some of our liquid ion-exchange chromatographic procedures to permit a greater sample throughput and, possibly, more efficient separations.

Thomas J. Murphy, Group Leader; I. Lynus Barnes, Ellyn S. Beary, William A. Bowman, Karen A. Brletic, Lura P. Dunstan, John D. Fassett, John W. Gramlich, Billy R. Hardas, Emerson F. Heald, William R. Kelly, Howard M. Kingston, George M. Lambert, Lawrence A. Machlan, John R. Moody, Paul J. Paulsen, P. Andrew Sleeth 


\section{Atomic and Molecular Spectrometry Group: Overview}

The goals of the Group continue to be closely associated with the ever increasing demands for more accurate, more sensitive, and more rapid spectrometric analyses. Currently, a multifaceted approach is being used which includes the development of improved plasma emission and atomic absorption instrumentation, investigation of controlled spectrochemical excitation, development of novel sample preparation and dissolution techniques, and the issuance of more useful spectrophotometric transmittance standards.

The Group's research efforts in atomic spectrometry have focused primarily on the continued development and improvement in the analytical capabilities of the inductively coupled plasma (ICP) spectrometer and on the evaluation of a newly acquired direct current plasma (DCP)/echelle spectrometer. Application of the ICP to the analysis of selected Standard Reference Materials has demonstrated that a variety of chemical and physical matrix interferences must be better understood and controlled before this technique can provide data sufficiently accurate for certification use. Studies with the DCP instrument have indicated that detection limits are comparable to the ICP and, through use of a lithium buffer solution, matrix interferences can be reduced to acceptable levels.

In molecular spectrometry, research efforts have been directed towards identifying those factors responsible for the apparent inhomogeneity observed with a significant number of the metal-on-quartz transmittance filter standards. The apparent inhomogeneity observed of approximately half of the chromium-on-quartz filters commercially produced for NBS is a problem that continues to severely hinder the widespread issuance of these filters as SRM's. For selected filters, transmittance differences as large as two percent have been observed when these filters are displaced laterally, $<1 \mathrm{~mm}$ with respect to the incident radiation. The recent transfer of the fabrication of these filters from an outside laboratory to the NBS Optical Shop should facilitate a more systematic study of the problem since optical measurements can be made at key stages in the fabrication process.

Paralleling these research efforts have been the demands associated with an intensive sample analysis work load. Areas in which the Group has been a principal contributor include Standard Reference Materials, hazardous waste characterization, Chesapeake Bay survey, used and recycled oil analysis, ppb analyses of injectables for the Food and Drug Administration, NBS-EPA National Environmental Specimen Bank, and service analyses.

Important accomplishments of the Group during the past year include: (1) design and construction of an improved extractor for the characterization of potentially hazardous wastes; (2) further refinement in the definitive method for sulfur in iron-base alloys; (3) development of an improved atomic absorption method for lead in blood; (4) performance of metal homogeneity measurements, using emission spectroscopy, with a relative standard deviation of 0.2 percent; (5) development of a graphite 
furnace technique for arsenic and selenium that is much superior to hydride generation; and (6) development of an ultratrace method for silicon utilizing the reaction with methane in conjunction with Zeeman atomic absorption spectrometry.

Future plans include the placing of a greater emphasis on understanding and controlling the fundamental excitation processes occurring in atom-producing sources, the systematic investigation of electrothermal ionization techniques for the direct analysis of solids, and further refinements in the multielement capabilities of the ICP, DCP, and continuum source wavelength modulated atomic absorption instrument. In addition, a major effort will be initiated within the Group in 1981 for performing metal speciation measurements by coupling high performance liquid chromatography with a variety of metal-specific spectrometric detectors.

Robert W. Burke, Group Leader; John R. Baldwin, Carol G. Blundell, David E. Brown, Therese A. Butler, Erle R. Deardorff, Barry I. Diamondstone, Michael S. Epstein, Shigeki Hanamura, Jerry D. Messman, Klaus D. Mielenz, John A. Norris, Theodore C. Rains, Theresa A. Rush, Robert L. Watters, Glenn J. Allin, Radu Mavrodineanu 
B. Selected Technical Accomplishments

(Inorganic Analytical Research Division)

1. D.C. Plasma Emission Spectrometry

The development of plasma sources for atomic emission spectrochemical analys is has revived interest in the technique as a rapid, precise, and sensitive method of sequential or simul taneous multielement determinations. While most analytical interest has been directed towards inductivelycoupled plasmas (ICP), recent design advances in direct-current plasma (DCP) technology have spurred development of that source.

The recent acquisition of a Spectraspan III D.C. Plasma/Echelle atomic emission spectrometer has not only significantly expanded our analytical capability, but has also provided us with an opportunity to study and improve the performance of the DCP. Two significant characteristics of a D.C. Plasma/Echelle spectrometer are its tolerance of a wide variety of organic solvents and the excellent $(\sim 0.005 \mathrm{~nm})$ spectral resolution. Recent investigations have taken full advantage of both these characteristics.

The direct determination of silicon in lubricating oil is preferred to oxidative digestion methods, primarily because time-consuming and contamination-prone procedures are avoided. The direct atomic absorption method involves introduction of an organic solvent containing the oil into a nitrous oxide/acetylene flame, a rather hazardous procedure. For this reason, DCP methodology was evaluated for the direct determination of silicon in a Wear-Metals-in-0il Standard Reference Material. Three proposed solvent systems for dissolution of the oil were investigated: xylene, kerosene, and methyl-isobutyl ketone (MIBK). MIBK was discarded because of its rapid attack on the peristaltic pump tubing. Xylene was satisfactory in that it dissolved both the oil and the organometallic Standard Reference Material (1066a--Octaphenylcyclotetrasiloxane) but it produced a significantly greater spectral continuum in the plasma than kerosene, in which the SRM 1066a was insoluble. A solvent system of 95/5 kerosene/xylene was finally chosen (with dissolution of the SRM 1066a in pure xylene and subsequent dilution with kerosene) and standards were matrix-matched to the dissolved oils using Conostan 245 basestock 011. The matrix-matching was required to avoid mixing chamber equilibration problems. The limit of detection was $40 \mathrm{ng} / \mathrm{mL}$ silicon and analytical results were within two percent of the certified value of the SRM WearMetals-in-0il with a relative standard deviation varying from one to two percent.

The analysis of refractory elements in high alloy steel by emission spectrometry is fraught with difficulties. High temperature plasma techniques produce a combination of intense continuum and complex line spectral radiation from sample concomitants. This spurious radiation severely tests the background correction capability and resolution of the spectroscopic instrumentation required to obtain arcurate analytical results. Figure 10 illustrates the importance of the resolution and background correction of the DCP/Echelle spectrometer for the determination 

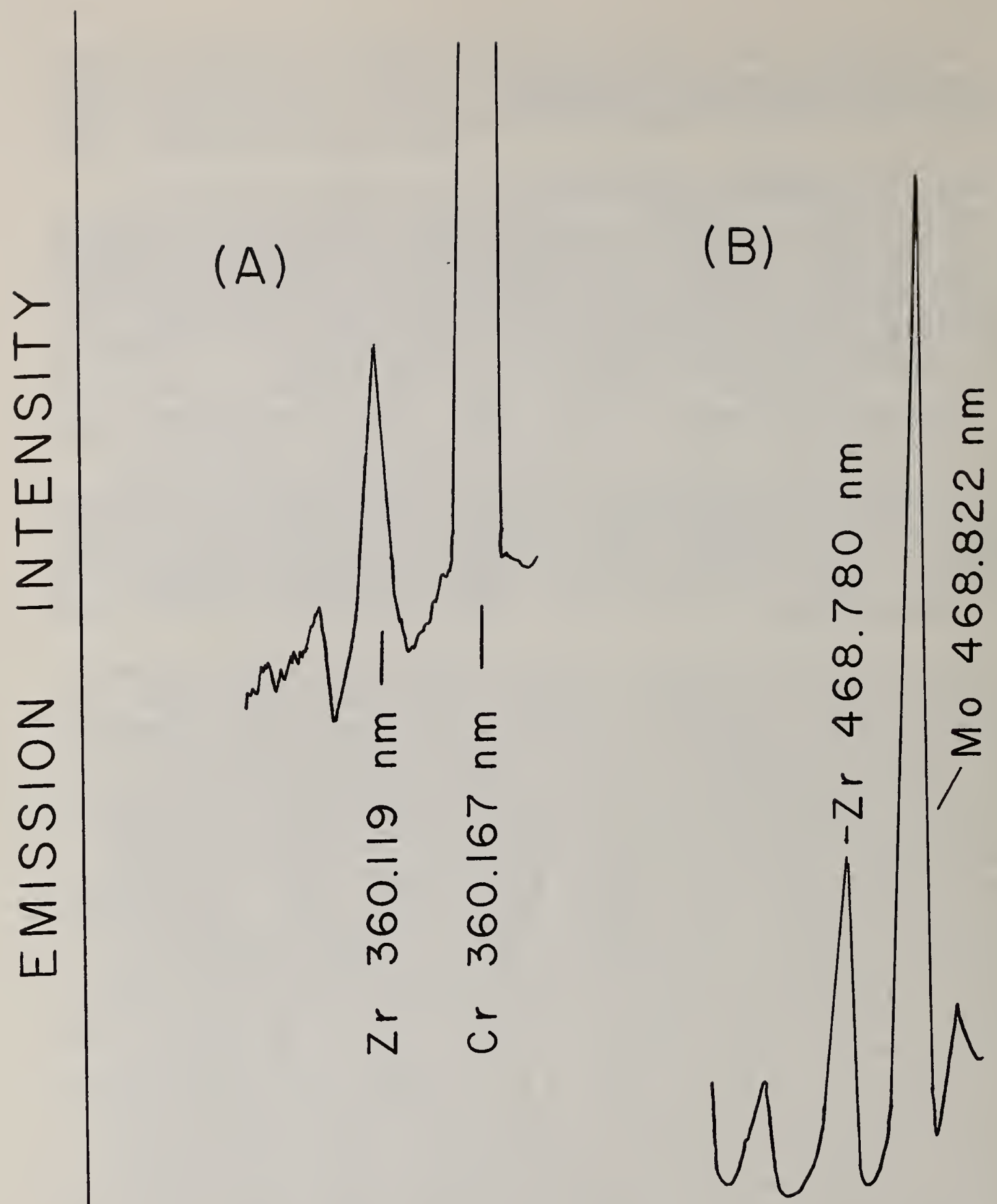

\section{WAVELENGTH}

Figure 10. Spectral wavelength scans of approximately $1 \mu \mathrm{g} / \mathrm{mL}$ zirconium in a matrix of one percent high alloy steel. While both zirconium analytical lines illustrated in $(A)$ and $(B)$ are useful, greater precision and accuracy are obtained using (B) because of the lower continuum radiation intensity. 
of zirconium in high alloy steel. Correlation of results from two analyte 1 ines, such as the $360.12 \mathrm{~nm}$ and $468.78 \mathrm{~nm}$ zirconium lines, provides a good assurance that accurate results have been obtained from the background correction procedures employed.

A program is presently underway to establish benchmarks (i.e., detection limits, precision, linear dynamic range, and accuracy) against which future system improvements can be measured. Some proposed modifications include: (a) improved pneumatic and ultrasonic nebulization for increased sensitivity and reduced chemical interference; (b) direct coupling to a liquid chromatograph for speciation studies; (c) application of gas generation procedures for sample introduction to improve sensitivity for elements such as boron, phosphorus, lead, arsenic, selenium, and other hydride forming species; (d) investigation of the use of the DCP as a radiation source for atomic fluorescence spectrometry; and (e) determination of the usefulness of the DCP as a source for vacuum UV emission spectrometry.

\section{S. Epstein}

\section{The Halogen Content of Lubricating 0 ils}

With the escalating emphasis on energy-efficiency, fuel saving and recovery, and material durability and reutilization, the halogen content of oils is of mounting importance and concern. It is vital to know that recycled oils burned in heating and power plants do not contain excessive amounts of halogen in order to limit acidic emissions; that re-refined lubricating oils have not been contaminated with halogen-containing cutting oils which could cause undue engine wear. Present analytical methods adopted by the American Society for Testing and Materials, the American National Standards Institute, and the Institute of Petroleum do not adequately address the problem at the levels of concern, being deficient in trace level capability, and in ability to distinguish chloride from bromide. Activation analysis, of course, can meet the demand and is being used extensively at NBS for this purpose, but this technique is costly and not readily available to commercial laboratories. The need for other methods is obvious.

With this end in mind, a new method has been developed capable of detecting chloride and bromide in oils at levels below $10 \mathrm{ppm}$. The method is based on a sodium alcoholate digestion, wherein halogen is liberated from its organically-bound state by a substitution reaction, an extraction of the free halide into water, and detection and quantitation by ion-chromatography. The digestion step is a variation of an ASTM procedure (D1317), but much less sample is required in the improved procedure. A serious emulsion problem can arise in the extraction step with some oils; it can be remedied with the aid of demulsifiers. The aqueous phase of the extract is treated with cation-exchange resin before injection into the ion-chromatograph to neutralize the base formed in the digestion step. Chromatographic conditions have been selected to optimize resolution of and sensitivity for chloride and bromide. A standard additions sequence is recommended to compensate for matrix effects on peak shape and retention time. Results, representative of the method's 
capability, are tabulated below. The present limitations in this new method are due primarily to the purity of the sodium metal used in the digestion step. The purest commercially available sodium contains $10-20 \mu \mathrm{g} / \mathrm{g}$ chlorine.

Whereas this new method cannot compete with activation analys is in terms of minimum detection limits (or precision at the lower levels), it should prove to be a valuable analytical tool for the rapid screening of oils for halogen content and for the quantitative determination of chloride and bromide in oils at levels above $10 \mathrm{ppm}$.

Table 3. Chlorine and Bromine in 0ils by Sodium Alcoholate/ Ion Chromatographic Method.

\begin{tabular}{|c|c|c|c|c|c|}
\hline $0 i 1$ & $\mathrm{ppm} \mathrm{Cl}$ & S.D. & $\underline{n}$ & $\mathrm{ppm} \quad \mathrm{Br}$ & S.D. \\
\hline 0033 & 225 & 34 & 4 & $<2$ & \\
\hline 0026 & 13.5 & 3.7 & 5 & 4.2 & 2.8 \\
\hline 0076 & 9.3 & 3.4 & 5 & 3.2 & 0.7 \\
\hline 0050 & 8.3 & 2.0 & 4 & $<1$ & \\
\hline $1634 a$ & 27.3 & 2.9 & 11 & $<1$ & \\
\hline
\end{tabular}

W. F. Koch

3. Measurement of the Co-60 to Co-59 Ratio in Microparticles

As part of the Bureau's health physics program, several metallic particles were discovered that had very high specific activities of ${ }^{60} \mathrm{Co}$; Division members were asked to help analyze them. The question raised was whether or not this activity could have been produced in the NBS reactor, and if so, how long ago. The measurement of the cobalt isotopic ratio by neutron activation analysis can contribute to a simple and effective means to identify the origin of the irradiated particle as well as to establish the approximate time or irradiation. As a result of the measurement described below, it was concluded that the particles were indeed produced in the NBSR about six years ago.

For example, if a cobalt-bearing steel is subjected to neutron irradiation and at a subsequent time the ratio of cobalt-60 to cobalt-59 atoms and the ratio of iron-55 to iron-54 atoms are measured, it is possible to determine both the decay time since the end of irradiation and the neutron fluence to which the particle was exposed. For this purpose, we developed two methods for the determination of the cobalt ratio and applied them to particles ranging in mass from $80 \mathrm{ng}$ to $300 \mu \mathrm{g}$. The measurements were carried out at the ORR reactor in Oak Ridge during the period of the NBSR shutdown. 
The first method is the approach of conventional neutron activation analysis. The sample was irradiated along with a known mass of el emental cobalt as a standard. The $58.6 \mathrm{KeV}$ gamma emitted by the 10.47 minute $60^{\mathrm{m}} \mathrm{Co}$ activity produced was counted using a thin Ge photon detector. This gave the ${ }^{59} \mathrm{Co}$ mass. The number of $60{ }^{\circ} \mathrm{co}$ atoms was determined by counting the particle against a ${ }^{60} \mathrm{Co}$ standard source (SRM-4210). This gave the specific activity of the particle as curies of $60 \mathrm{Co} / \mathrm{gram}{ }^{59} \mathrm{Co}$.

The direct measurement of the ${ }^{60} \mathrm{Co} / 59 \mathrm{Co}$ ratio was also done by NAA. For this, a know mass of cobalt was irradiated along with a ${ }^{60} \mathrm{Co}$ source of known activity. In addition to the ${ }^{59} \mathrm{Co}(n, \gamma){ }^{60 \mathrm{~m}} \mathrm{Co}$ gamma, we also measured the activity of the $67.4 \mathrm{KeV}$ gamma from the 99 minute 61 co produced by ${ }^{60}$ Co $(n, \lambda)^{6}$ Co. This provided a calibration of the irradiation/ counting system since

$$
\frac{N_{60}}{N_{59}}=\frac{A_{0}^{6 I}}{A_{0}^{60 m}} \quad \frac{\sigma_{59} \mathrm{I}_{60} \mathrm{~m} \cdot \varepsilon_{60} \mathrm{~m}}{\sigma_{60} \Gamma_{61} \varepsilon_{61}}
$$

where

$$
A_{0} \equiv \frac{\lambda c e^{\lambda t_{1}}}{\left(1-e^{-\lambda \tau}\right)\left(1-e^{-\lambda \Delta}\right)} \cdot \frac{e^{\lambda \delta}-1}{\lambda \delta}
$$

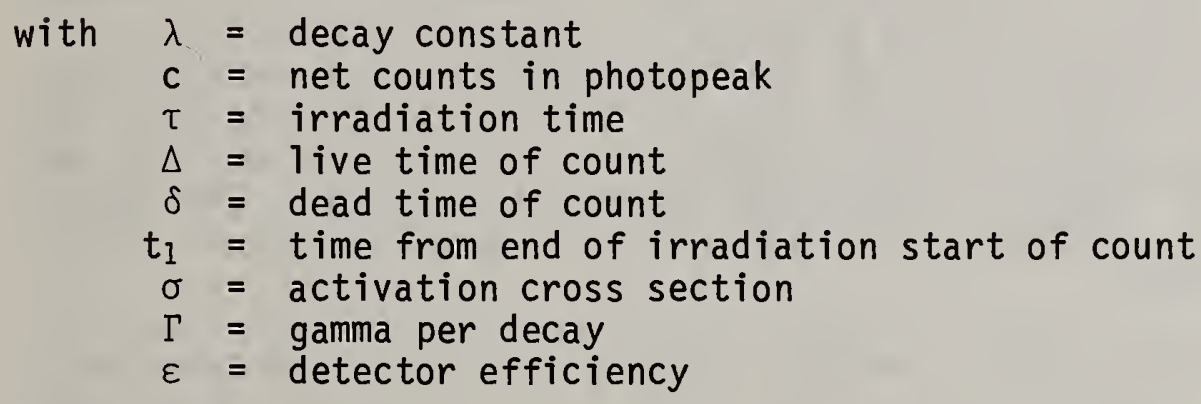

The measurement gave the calibration factor (in square brackets) as 0.229 ( \pm 10 percent) so that the specific activity is given as

$$
\text { Curies }\left({ }^{60} \mathrm{Co}\right) / \text { grams }\left({ }^{59} \mathrm{Co}\right)=264 \frac{\mathrm{A}_{0}^{61}}{\mathrm{~A}_{0}^{60} \mathrm{~m}}
$$

This second method has the important advantage that all geometry effects during irradiation and counting are eliminated. This proved to be crucial in the case of particle DIOA which was not visible and hence difficult to position precisely. The results of the measurements for six particles by the two methods are given below. The agreement is excellent except for $D 10 A$ for which the direct method gives the more reliable result. 


\begin{tabular}{|c|c|c|c|c|}
\hline Particle & Weight $(\mu \mathrm{g})$ & $\begin{array}{l}{ }^{60} \text { Co Activity } \\
(\mu \mathrm{Ci})\end{array}$ & $\begin{array}{r}C i \\
\text { Direct } \\
\end{array}$ & $\begin{array}{l}0 / \mathrm{gm}{ }^{59} \mathrm{Co} \\
\text { Conventional }\end{array}$ \\
\hline G2 & 300.3 & 12.8 & -- & 0.91 \\
\hline DI & 66.2 & 9.30 & 1.36 & 1.34 \\
\hline RF & 28.0 & 8.97 & 10.4 & 10.2 \\
\hline G1 & 15.7 & 5.58 & 11.6 & 11.6 \\
\hline HEX 7-1 & 134.3 & 257 & -- & 19.6 \\
\hline D10A & 0.083 & 2.59 & 335 & 282 \\
\hline
\end{tabular}

B. S. Carpenter, R. F. Fleming, R. M. Lindstrom, R. Zeisler

\section{Cryogenic Homogenization of Tissue}

The major task of the National Environmental Specimen Bank (NESB) Pilot Program includes the evaluation of specimen storage under different conditions, and the analysis and re-analysis of the same specimen by different techniques. To obtain the needed information, the individual specimens have to be stored and analyzed in the form of small but representative subsamples. Large inhomogeneities within a single liver have been reported when typically one gram subsamples are used [1]. The concentrations of essential trace elements vary by a factor of four and up to a factor of 1000 for other trace elements. Therefore, the ability to homogenize the tissue to produce representative subsamples is essential to the program.

The method of cryogenic homogenization [2], brittle fracture technique, has been adapted to the needs of the banking program and has been evaluated for its performance and general applicability to the homogenization of fresh, soft biological material. A prototype brittle fracture with up to $250 \mathrm{~g}$ capacity has been developed at NBS. This capacity is needed to homogenize a half left lobe of a human liver, one of the kinds of specimens stored in the NESB. The machine is essentially a Teflon ball mi11, in which samples are fractured and homogenized at 1 iquid nitrogen temperature without contamination.

The storage evaluation experiment of the NESB Pilot Program requires that the inhomogeneity error due to the analytical subsample is less than 10 percent. To evaluate the homogeneity and the performance of the modified brittle fracture technique, the sampling constant and sampling diagram models [3] have been applied. The models developed for particulate materials assume Poisson statistics to govern the relation between sample size and sampling error. A sampling constant can be defined as

$$
R=\sqrt{\frac{K_{s}}{W}} \quad \text { or } K_{S}=R^{2} W
$$


where $R$ is the relative error in percent and $w$ is the sample weight. Therefore, $K_{s}$ represents the sample weight, at which the sampling error is one percent. For nearly particulate analytical materials, e.g. liver homogenate, the sampling constant can be estimated by generating a sampling diagram and examination of its compliance with the above assumption. A sampling diagram can be developed by analyzing a sufficient number of subsamples of different weights. The analytical error necessarily has to be small, at least less than $1 / 3$ of the sampling error.

Radiotracer studies can provide the required analytical information; however, to minimize possible different behavior of spikes, a five gram subsample of a liver specimen was irradiated ( 2 min at $5 \times 10^{13} \mathrm{ncm}^{-2} \mathrm{~s}^{-1}$ ). The radioactive subsample was added to the remainder of the tissue and then homogenized. Ten subsamples each were taken at a nominal weight of $100 \mathrm{mg}, 1000 \mathrm{mg}$, and $6000 \mathrm{mg}$. Subsequently, the subsamples were counted on a gamma spectrometer. Counting errors were minimized to the error due to counting statistics.

The radioactive ${ }^{24} \mathrm{Na}$ was used to monitor the distribution of sodium in the samples. The results are plotted in figure 11 , showing a typical sampling diagram. The sampling constant, $K_{s}$, i.e., the subsample weight where an inhomogeneity of one percent can be expected, was calculated to be $35 \mathrm{~g}$. This experiment confirms our ability to take a grossly inhomogeneous sample and produce a material from which representative subsamples can be taken. With a typical analytical subsample of one gram used in the NESB Program, a sampling error of five percent or less can be expected. The upgrading of the brittle fracture technique and the measurable improvements of the homogeneity of the specimens will have impact on the trace analysis in life sciences and environmental sciences. A large sample can now be homogenized without the danger of contamination by metals and fluids used in other blending operations. In addition, the sample is kept at low temperatures throughout the entire operation, thus largely preventing chemical and biochemical changes. 


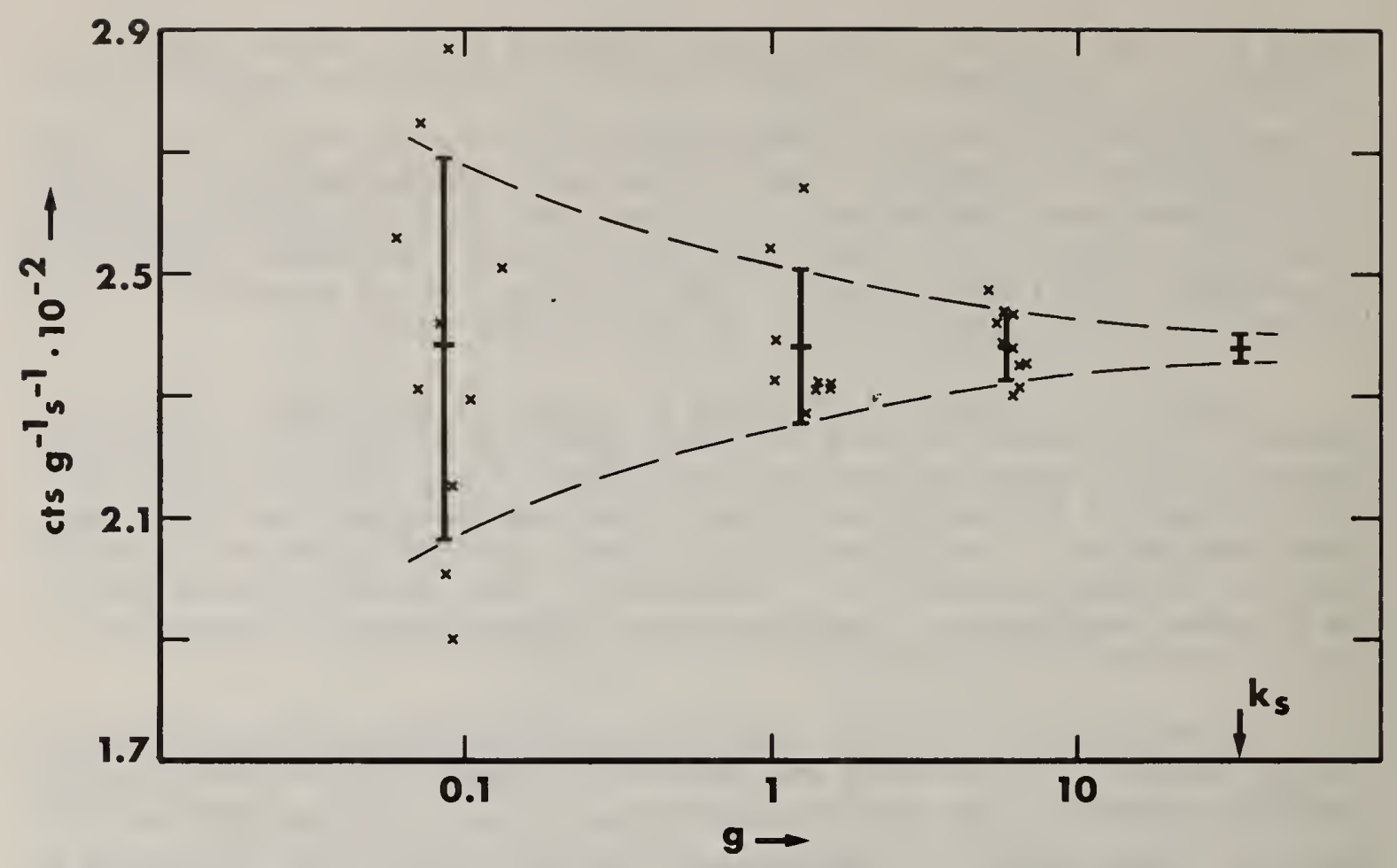

Figure 11 . Sampling diagram of ${ }^{24} \mathrm{Na}$ in human liver homogeneate.

References

[1] Schicha, H., Kasperek, K., Riedel, V., Feinendegen, L. E., Vyska, K., and Müller, W., Proc. Symp. Nuclear Activation Techniques in the Life Sciences 1972, IAEA, Vienna, 451, (1972).

[2] Iyengar, G. V. and Kasperek, K., J. Radioanal. Chem., $\underline{39}, 301$ (1977).

[3] Ingame11s, C. 0., Geochim. Cosmochim. Acta, 38, 1225 (1974).

S. H. Harrison, R. Zeisler

5. Rapid Analysis of Toxic Metals in Biological Tissue and Fluids by Atomic Absorption Spectrometry

Current interest in the effect of trace quantities of toxic metals in plants, animals, and human beings, coupled with widespread concern over the possible accumulation of these toxic metals in the environment, has created a need for the determination of ever lower concentrations of metals in a wide variety of biological materials. Reliable methods do not exist in many clinical or biological laboratories for the determination of physiological levels of many trace metals. The ideal clinical or biological 
method should be relatively rapid, free from interferences, capable of determining relatively low concentrations of the analyte in a minimum volume of sample, and have a relativeiy high degree of precision and accuracy. Unfortunately, Standard Reference Materials for trace metals in biological fluids are not available and only two types of animal tissue are available to evaluate analytical methods currently being used in these laboratories.

In the area of blood-1ead, a method was developed at NBS whereby lead is stripped from $100 \mu \mathrm{L}$ of whole blood into 5 percent nitric acid medium and determined by AAS with electrothermal atomization. The method is capable of determining 5-90 $\mathrm{gg} \mathrm{Pb} / \mathrm{dL}$ of whole blood with a precision of $\pm 2 \mu \mathrm{g} / \mathrm{dL}$. The time required for an analys is is 15 minutes. The method developed at NBS is being widely used in clinical laboratories throughout the U.S.A. and the world. The technique was recently adopted as the standard method for blood-lead at the Mayo Clinic, Rochester, MN. The analytical capability of the method is tremendous. Cadmium in whole blood can be determined with a precision that is comparable with the blood-lead analysis. This method meets the criteria for an ideal clinical method.

In the analysis of biological tissue and bio-injectible vaccines by conventional AAS methods, cadmium and lead signals are severely suppressed. To alleviate this difficulty, a technique of matrix modification is used. For lead analysis, a 0.01 percent $\mathrm{K}_{2} \mathrm{Cr}_{2} \mathrm{O}_{7}$ solution is added and the sample is charred at $900{ }^{\circ} \mathrm{C}$ in the electrothermal atomizer prior to atomization. The high charring temperature removes many of the interferences without loss of the lead. In cadmium analysis, the interferences are alleviated by the addition of a 1 percent solution of ammonium orthophosphate $\left[\left(\mathrm{NH}_{4}\right)_{2} \mathrm{HPO}_{4}\right]$. In the presence of $\left(\mathrm{NH}_{4}\right)_{2} \mathrm{HPO}_{4}$, the sample can be charred at a temperature of $800{ }^{\circ} \mathrm{C}$ without loss of cadmium. The difficulty encountered with the use of $\left(\mathrm{NH}_{4}\right)_{2} \mathrm{HPO}_{4}$ is a high reagent blank. To overcome this difficulty, the $\left(\mathrm{NH}_{4}\right)_{2} \mathrm{HPO}_{4}$ is pre-extracted with a solution of ammonium pyrrolidine dithiocarbamate (APDC) and methyl isobutyl ketone.

With an increased interest in the trace analysis of arsenic and selenium in biological tissue and fluids, an evaluation of several analytical techniques was undertaken. The vapor generation-AAS technique using an argon (entranced air)-hydrogen flame was found to produce low and erratic results. The system was modified by using a heated absorption cell with no resulting improvement in interferences. Electrothermal atomization was investigated using matrix modification by adding $1000 \mu \mathrm{g} \mathrm{Ni} / \mathrm{mL}$. The addition of nickel permitted a charring temperature of $800{ }^{\circ} \mathrm{C}$. This method was found to alleviate the interferences encountered in biological tissue and fluid. As a check of the recovery of the method, the single standard addition technique was used.

Currently, AAS with electrothermal atomization is being used to survey trace metals ( $\mathrm{Al}, \mathrm{As}, \mathrm{Ba}, \mathrm{Cd}, \mathrm{Co}, \mathrm{Cr}, \mathrm{Hg}, \mathrm{Pb}, \mathrm{Se}, \mathrm{Tl}$, and $\mathrm{Zn}$ ) in several types of injectable biological products. As a result of this survey, a biological product will be selected as a candidate for a Standard Reference Material.

C. G. Blunde11, T. A. Butler, J. D. Messman, T. C. Rains, T. A. Rush 
6. Comparative Measurements of Zinc-70 Enrichment in Human Plasma Using Neutron Activation Analys is and Thermal Ionization Mass Spectrometry

A joint project between the Inorganic Analytical Research Division and the Nuclear Reactor Laboratory, Department of Nutrition and Food Science, Massachusetts Institute of Technology was undertaken to establish whether analytical methodology is available that would allow accurate measurement of ${ }^{70} \mathrm{Zn}$ enrichment in human plasma during zinc metabolism studies. If successful, it would be possible to conduct human zinc metabolism studies with small amounts of $70 \mathrm{Zn}$ stable isotope instead of either radioactive zinc or abnormal quantities of natural zinc. The two methods chosen for this investigation were radiochemical neutron activation at MIT and thermal ionization mass spectrometry at NBS on samples provided by MIT.

Plasma appearance curves for zinc following oral administration in humans have been studied to a very limited extent using both radioactive zinc isotopes and natural zinc. The former method is restricted in regard to general use because of issues related to administration of radioisotopes, while the latter requires oral intakes of zinc significantly greater than the normal daily intake of the mineral.

The stable isotope $70 \mathrm{Zn}$ is natually present at 0.62 percent atomic abundance, being the least abundant of all stable isotopes of zinc. Therefore, it could potentially provide a suitable vehicle for studies of dietary enrichment. It is of considerable interest and importance to determine whether or not oral administration of $70 \mathrm{Zn}$ at physiologically relevant levels would allow the measurement of zinc enrichment in human plasma to the extent that would permit the investigation of plasma appearance kinetics for zinc.

Accurate measurements of $70 \mathrm{Zn}$ enrichment under normal nutritional conditions is difficult due to two primary factors. First, in a typical human study involving time-dependent measurements, the amount of plasma available at any given time is restricted to only a few milliliters. This sample size provides a total $70 \mathrm{Zn}$ content of only a few nanograms. Secondly, the expected total plasma increase due to the administration of the isotope is at most only a few percent of the oral dose.

Following an overnight fast, four healthy adult male volunteers consumed a single glass of orange juice containing $3.2 \mathrm{mg}$ of ${ }^{70} \mathrm{Zn}$. Venous blood samples were withdrawn from the anticubital vein every fifteen minutes for the first two hours, at half hour intervals for the next $11 / 2$ hours and then hourly until eight hours after isotope ingestion. The blood samples were analyzed by neutron activation analysis at MIT and by mass spectrometry at NBS.

Previously employed mass spectrometric procedures for zinc isotopic analysis required approximately 10 micrograms of zinc per analysis. Since these plasma samples contained only about 0.5 micrograms of zinc, it was necessary to develop mass spectrometric techniques for the analysis of submicrogram quantities. Modifications to the existing 
procedure, principly lowering the zinc-to-silica-gel ratio on the filament, achieved the goal of allowing $68 \mathrm{Zn} / 70 \mathrm{Zn}$ ratios to be measured on 0.3 microgram samples with a precision for replicate analyses of a standard of 0.13 percent relative ( 1 std. dev.). The corresponding precision found for activation analysis was 8.3 percent.

The results of this study indicate that in a typical metabolic study, where a single $3 \mathrm{mg}$ dose of $70 \mathrm{Zn}$ is administered whose kinetic plasma appearance is similar to the data obtained in this study, then at the peak appearance the expected $70 \mathrm{Zn}$ plasma enrichment should be about 200 percent of the plasma ${ }^{70} \mathrm{Zn}$ baseline level (an increase in ${ }^{70} \mathrm{Zn}$ enrichment from 0.62 percent to 1.83 percent). Under these conditions, neutron activation analys is should provide satisfactory results with its attainable precision of 5-10 percent. If however, ${ }^{70} \mathrm{Zn}$ appearance is significantly lower than these values, then the precision of neutron activation analysis becomes marginal and a technique such as mass spectrometry will be required to obtain meaningful results.

J. W. Gramlich, L. A. Machlan, M. Janghorbani

\section{Trace and Toxic Elemental Study of the Chesapeake Bay}

As part of a multidisciplinary study of the Chesapeake Bay, the National Bureau of Standards (NBS) was asked to develop the techniques and procedures necessary to measure the trace and toxic element concentrations within the water column throughout the entire length of the Bay. As part of this program, NBS collected 102 separate water samples and is currently completing the analys is for selected elements ( $\mathrm{Cd}, \mathrm{Ce}, \mathrm{Co}, \mathrm{Cr}$, $\mathrm{Cu}, \mathrm{Fe}, \mathrm{Mn}, \mathrm{Mo}, \mathrm{Ni}, \mathrm{Pb}, \mathrm{Sc}, \mathrm{Sn}, \mathrm{Th},{ }^{238} \mathrm{U}, 235 \mathrm{U}$, and $\mathrm{Zn}$ ), including some elements at concentrations consistently below one picogram per milliliter (part per trillion). The collection of the samples was described in the 1979 Technical Activities, however, the list of elements to be analyzed was upgraded this year to its present complement with the addition of $\mathrm{Ce}, \mathrm{Sc}$, Th, and U.

The literature on marine water analysis reflects the considerable difficulty in establishing an accurate and precise method of analysis for trace metals. An estuarine or sea water matrix defies a simplified approach. The extremely low trace concentrations cause the sample and analytical blank to be of paramount importance. The integrity of the sample can be compromised by just a brief exposure to normal laboratory air. In addition, the extremely high concentrations of alkali, alkaline earth, and halogen elements in the marine water matrix make direct analysis difficult or impossible for most analytical techniques.

To circumvent these problems, special chemical and instrumental procedures were developed. While the chemical separation, preconcentration was completed in late 1979, it was optimized this past year for neutron activation analysis (NAA). The basic chemical procedure was developed and published in 1978 and $1979[1,2]$. The chemical procedure was substantially unchanged as reported for use with thermal device atomic 
absorption (TDAA). However, the elements were not eluted from the resin when used in conjunction with NAA. Both the modification and the established procedure proved to be extremely advantageous when applied to estuarine or seawater analysis.

The elimination of matrix elements allows the determination of many elements which could not otherwise be analyzed and enhances the sensitivity of other elements of interest. The control of the blank in this procedure has enabled its contribution to be sufficiently low that it does not limit the measurement of pristine samples. The particulate fraction of each sample was prepared in the field by splitting a two-liter sample into two one-liter filter samples, one for TDAA and a second for NAA to eliminate handling and dividing a single particulate sample.

Both the samples and the blanks have undergone rigorous statistical scrutiny and are being reported after correction for blank contribution (where necessary) at the 95 percent confidence limit.

It was necessary to extend the capabilities of the chemical and instrumental techniques to obtain state-of-the-art concentration data for the samples. All of the elements of interest cannot be analyzed by a single instrumental technique. The methods necessary to analyze the estuarine and seawater using NAA have been developed this past year. The combined use of NAA and TDAA provides the capability to determine a wide range of important trace elements in estuarine samples. It was found that by drying the resin in the column after chemical separation and analyzing it directly in heat sealed polyethylene film that NAA could simultaneously analyze many elements at the ultratrace level with a high degree of accuracy. This detailed technique will be published in the open literature in the near future.

These concentration measurements will be used as the basis for several studies. Because the samples were taken concurrently with sediment, pore water, and benthic studies, both the water-to-sediment equilibrium and aquatic population will be correlated. Beyond this, a "state-of-the-Bay" is being established which includes an estimate of the impact of the population area impinging on it. The ultimate goal of the program is to produce an informative study to guide the management of the Bay. To meet these demands, it is necessary to compare the results respective of their location throughout the Bay. The interpretation of the concentration data obtained at the different locations throlighout the Bay is extremely difficult. At a single location the concentration can vary with time, tide, current, wind conditions, temperature, bints, etc. With these perturbations of concentration at a single lc_.... on, interpretation of the differences found at separate locations is formidable. One approach which has proven useful in minimizing fluctuations is the comparison of elemental ratios rather than absolute concentrations. For this technique to be successful, however, the elemental concentration must be determined with a high degree of accuracy. Reference elements must be chosen which are ubiquitous and essentially nonanthropogenic. An increase in the total mass loading will increase the absolute concentrations, but will not affect the elemental ratios. A significant addition of a single element or several 
elements to the environment from either natural or anthropogenic sources will increase the ratios of this (these) element(s) with respect to the reference element. Increases in concentration, however, may not be obvious over the normal fluctuations of the absolute el emental concentrations. This technique has been applied to the elemental concentrations in the estuarine samples with dramatic results. The elemental concentrations in the particulate fractions were found to vary over two orders of magnitude between locations as indicated in Table 5 . The ratios for $\mathrm{Fe}$, $\mathrm{Th}$, and $\mathrm{Ce}$ are essentially constant, differing by only 20 percent, while the Co to Sc ratio is four times greater in sample 11,032 than in either 11,005 or 11,007 .

Table 5. Concentration in Particulate Phase and Ratios to Scandium.

Sample Number

$$
11,005 \quad 11,007 \quad 11,032
$$

Element

Concentration--ng/mL

\begin{tabular}{lrlrlll} 
Fe & \multicolumn{1}{rl}{2,800} & \pm 100 & 128 & \pm 8 & 18 & \pm 1 \\
Co & 1.02 & \pm 0.05 & $0.050 \pm 0.003$ & 0.028 & \pm 0.003 \\
Sc & 0.90 & \pm 0.005 & $0.040 \pm 0.002$ & $0.0049 \pm 0.0002$ \\
Th & 0.66 & \pm 0.03 & $0.032 \pm 0.002$ & $0.0038 \pm 0.0005$ \\
Ce & 6.0 & \pm 0.3 & $0.26 \pm 0.01$ & $0.039 \pm 0.003$
\end{tabular}

Ratio to Scandium, $[\mathrm{X}] /[\mathrm{Sc}]$

$\mathrm{Fe}$

$3.2 \times 10^{3}$

$3.2 \times 10^{3}$

$3.7 \times 10^{3}$

Co

1.1

1.3

5.7

Sc

$\equiv 1$

$\equiv 1$

$\equiv 1$

Th

.73

.80

$\mathrm{Ce}$

6.7

6.5

8.0

This technique provides a means for identifying the similarities and differences between the sample locations. A comparison of ratios throughout the Bay may be useful in identifying anthropogenic and/or naturally occurring sources to the Chesapeake Bay. If the concentrations of toxic elements are found to be excessively high, it is necessary to identify their sources before any attempt can be undertaken to reduce these levels 
[1] Kingston, H. M., Barnes, I. L., Brady, T. J., Rains, T. C., and Champ, M. A., "Separation of Eight Transition Elements from Alkali and Alkaline Earth Elements in Estuarine and Seawater with Atomic Absorption Spectrometry," Anal. Chem., 50, 2064 (1978).

[2] Kingston, H. M., "Quantitative U1tratrace Transition Metal Analysis of High Salinity Waters Utilizing Chelating Resin Separation," Interagency Energy/Environmental R\&D Program Report, P. 72, EPA-600/7-79-174. 1979.

E. S. Beary, R. R. Greenberg, H. M. Kingston, T. C. Rains

8. Characterization of Potentially Hazardous Wastes-Development of Rotary Extractor and Quality Assurance Standards

With the passage of the Resource Conservation and Recovery Act in 1976, Congress mandated that EPA establish a set of regulations by April 1978 regarding the identification, transportation, and disposal of hazardous waste materials. EPA subsequently defined eight characteristics for waste materials, any one of which would classify a waste material as hazardous. One of these characteristics, E.P toxicity, EPA has chosen to relate to the amounts of certain priority inorganic and organic pollutants which are leached from the wastes under a set of empirical extraction conditions:

Late in FY 79, NBS entered into an interagency agreement with EPA to evaluate the several extraction procedures that were being considered as acceptable tests for the identification of hazardous wastes.

In order for NBS to fulfill its obligation to EPA, it was necessary to develop a procedure which would reproducibly extract the leachable priority pollutants from the waste material. Although a number of extraction techniques are presently in use, the results of various interlaboratory studies indicate that variations in concentrations of orders of magnitude for the priority pollutants are not uncommon. Although this can be a result of poor analytical technique and/or sample inhomogeneity, the possibility of poor extractor designs must also be considered. In fact, data presented at a number of ASTM meetings in which several members of NBS staff participated, clearly indicated that the reproducibility of the extraction procedures was extremely poor. Thus, in order for meaningful measurements to be made on an interlaboratory basis, an improved extractor design was of paramount importance.

Based on our interpretation of the EPA Federai Guidelines, a model extractor was designed which allowed four samples to be run simultaneously in 2-liter containers. The sample containers were tumbled end-over-end at $29 \mathrm{rpm}$ permitting intimate contact between the waste and the extracting solution. Although extensive testing of this rotary extractor at NBS is 
incomplete, EPA has chosen to include this design as an acceptable model in the latest Federal guidelines. Presently, studies are in progress to evaluate the efficiency and abrasion characteristics of this design on selected wastes. An illustration of this rotating extractor is shown in figure 12 .

At the moment, NBS is in the process of providing to EPA a number of units of representative waste material which will be a fly ash that is totally characterized for chemical composition using NBS standards. Information will also be provided on the concentrations of the priority pollutants which can be leached from the material under specific extraction conditions. In addition, NBS is initiating studies on some sludge materials to be used as more complex quality assurance standards. Work in this area is expected to continue in FY 81.

As a parallel effort, NBS has provided the Department of Energy Laramie Energy Technology Center (DOE-LETC) with a set of quality assurance standards for the area of fossil fuel wastes. This effort has required the collection of ton-quantities of coal, coal fly ash, and coal bottom ash which were sieved, ground, blended, bottled, and delivered to DOE-LETC. As in the case of the EPA material, these samples were fully characterized for chemical composition of both the solid and the extract. Additionally, as part of the quality assurance program, two levels of synthetic leachate solution were prepared, each fully characterized for 18 inorganics. Work is expected to continue in these areas through FY 81 .

R. W. Burke, B. I. Diamondstone 


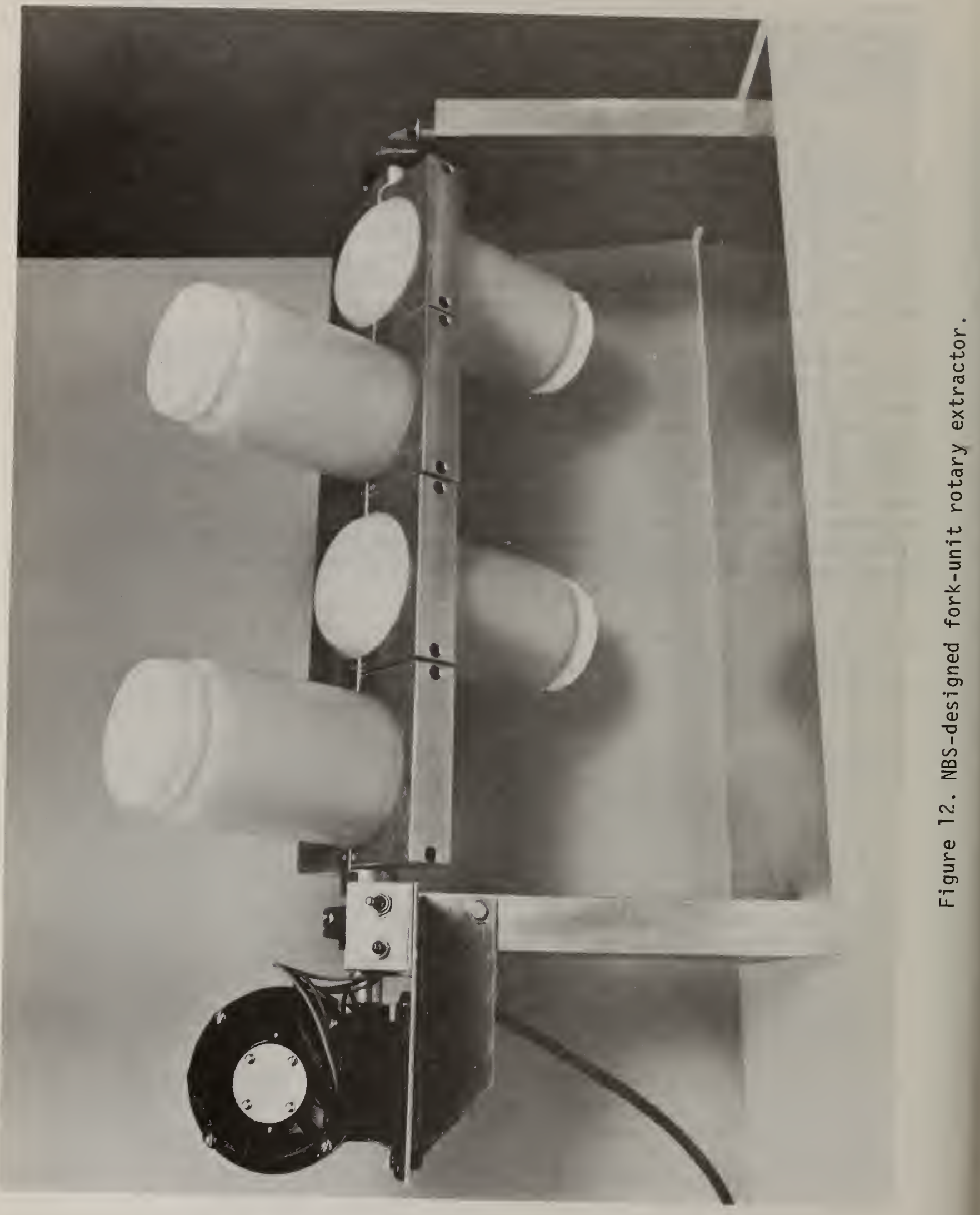


9. Development of an Improved Gravimetric Method for the Determination of Aluminum in Bauxite

Increasing numbers of reports of difficulties of many analysts in the determination of aluminum in bauxite and bauxite SRMs have led to an investigation that would result in a reference method for this analysis. This is a very important problem, because bauxite ore is the primary source for the production of aluminum.

There are several different types of bauxite, only one of which, Arkansas, is domestic to this country. The production of aluminum is tailored to the particular compositions of bauxite used in order to achieve the desired end product. Since the U.S. is not self-sufficient, we usually import Dominican, Jamaican, and Surinam bauxites for blends refined in this country. Bauxite SRMs and accurate definitive methods of analysis applicable to any of the different types are of prime importance in trade equity in the world market place and for process control in the manufacture of aluminum and aluminum-base alloys. Not only is the accurate knowledge of the aluminum content of the ore important for the methodology of production control and quality assurance program for efficient plant operation with attendant energy savings, but also, original ore content must be determinable within certain narrow fixed limits to satisfy the buyer-seller needs. Bauxite SRM's have been issued by NBS for at least 50 years to provide industry with a means of calibrating their methods.

Aluminum determinations in bauxite at the high concentrations present ( 250 percent $\mathrm{Al}_{2} \mathrm{O}_{3}$ ) have always presented difficulties because of the necessity to make quantitative separations. The task of repeated filtrations, the handling of the voluminous, gelatinous precipitates, boiling large volumes of solutions, without losses or contamination is laborous, onerous, and difficult even for experienced wet analytical chemists.

The chief sources of error for aluminum determinations in bauxitetype materials by the classical analytical ammonium hydroxide-cupferron precipitation includes: failure to completely decompose the material; failure to fuse the residue remaining after $\mathrm{HF}-\mathrm{H}_{2} \mathrm{SO}_{4}$ treatment to remove silica; failure to correct for compounds normally precipitated with ammonia; failure to correct for materials that are co-precipitated with the aluminum hydroxide; precipitation of the alkaline earths as carbonates; failure to remove alkali salts which are carried down with the aluminum hydroxide precipitate; incomplete re-dissolution of the hydroxide precipitate prior to the second reprecipitation; improper ignition of the hydroxide precipitate; failure to protect the ignited alumina from moisture before or during weighing; failure to correct for silica carried down by the hydroxide; and misleading blank corrections.

The Hoffman-Hillebrand ammonium-hydroxide-cupferron method is long and tedious, but attempts at short cuts have led to erroneous results. Some of the other separation techniques have been tried, such as ion exchange which failed as a result of overloaded columns. 
The investigation here involved some modifications of the HoffmanHillebrand method to el iminate some of the sources of error and include: slightly higher drying temperatures of the ore; additional fusions to get all of the residue in solution; electrolys is of the sample solutions with a mercury cathode; a single cupferron precipitation followed by a cupferron- $\mathrm{CHCl}_{3}$ extraction of the solution of the first precipitate; a triple rather than a double ammonium hydroxide precipitation; ignition in covered, platinum crucibles at $1200^{\circ} \mathrm{C}$ (this is done in the HoffmanHillebrand method but has been omitted by many recent analysts); evaporation of the residues to dryness with $\mathrm{HF}$ and $\mathrm{H}_{2} \mathrm{SO}_{4}$ to remove traces of silica; reignition at $1200^{\circ}$ to constant weight; examination of the alumina residues for contaminants by emission spectroscopy and subtracting the amount found from the alumina residue.

DTA studies of the bauxite SRM 69a indicated that samples should be dried at $170{ }^{\circ} \mathrm{C}$ to constant weight instead of at $140{ }^{\circ} \mathrm{C}$ as previously recommended. It was also observed that often two separate fusions with sodium carbonate and sodium pyrosulfate after filterings and ignitions were necessary to completely dissolve the residue. At additional steps in the procedure, a residue was sometimes observed, necessitating an additional filtration, ignition, and fusion. The mercury cathode electrolysis step was added to remove materials which are removed incompletely or not at all by the cupferron separation. These elements are: chromium; manganese, in part; zinc; silver; cadmium; indium; thallium; lead, in part; bismuth; platinum metals, in part; etc. It also removes the bulk of the iron, making the cupferron separation easier to handle. An extraction was substituted for the second cupferron precipitation to minimize the contamination from the slight solubility of the cupferrates. These cupferrates can be extracted more quantitatively than they can be precipitated. An extraction is not recommended for the first cupferron separation, because such a large amount of aluminum is present at that step, its mechanical loss in the process would be possible owing to pressure build-up in the extraction funnel. The triple ammonium hydroxide precipitation minimizes the contamination resulting from hydroxide co-precipitation, precipitation of alkaline earth carbonates, and the alkali salts carried down with the hydroxide. No problems with hygroscopicity were experienced when the crucibles were ignited, covered at $1200^{\circ} \mathrm{C}$. In the Hoffman-Hillebrand method, each alumina residue was analyzed individually and corrected for $\mathrm{SiO}_{2}, \mathrm{Fe}_{2} \mathrm{O}_{3}, \mathrm{TiO}_{2}, \mathrm{ZrO}_{2}, \mathrm{P}_{2} \mathrm{O}_{5}$, $\mathrm{Cr}_{2} \mathrm{O}_{3}$, and $\mathrm{V}_{2} \mathrm{O}_{5}$. With much less effort, emission spectroscopy can determine these constituents (with the exception of phosphorus) as well as barium, cobalt, magnesium, manganese, sodium, potassium, vanadium, tungsten, zinc, and zirconium, etc. A result of 55.03 percent was obtained for SRM 69 which was carried along as a control. This value should be compared with the average value of 55.00 percent obtained by J. I. Hoffman by three separate methods and its certified value of 55.06 percent and is consistent with the confidence limits for each of these.

\section{E. J. Maienthal}


One of the activities completed within the Division during the last fiscal year was the field testing and evaluation of a fission track reactor power monitor for the International Atomic Energy Agency (IAEA). The purpose of this task was to evaluate the capability of the tracketch monitor for providing an independent record of the nuclear reactor's power history and to provide information concerning the neutron energy spectrum.

The track-etch monitor evaluated was an externally-powered device designed to record the neutron flux from operating reactors and similar sources for extended periods of time. A strip of $35 \mathrm{~mm}$ wide polymer tape is drawn past a group of fissionable deposits which have been exposed to the local neutron flux. A fraction of the fission fragments generated penetrate the tape producing radiation-damaged tracks that are subsequently etched and counted to permit a reconstruction of the temporal flux history to which the monitor was exposed. Each monitor is equipped with sufficient tape to cover a year of reactor operation time. The monitor is designed to operate at a neutron flux of $10^{6} \mathrm{ncm}^{-2} \mathrm{~s}^{-1}$.

In one of the actual field tests, a monitor was installed above the shroud at the Maine Yankee Power Reactor in Wiscassett, Maine, for ten months. During this period of time, the 855 Megawatt reactor underwent four unscheduled shut-downs and over eight changes in reactor powerlevels. The 14.5 meter tape was removed from the monitor, chemically etched to reveal the fission tracks, which were measured. The investigation of the tape indicated that the monitor was able to record the changes in reactor power but had suffered several mechanical malfunctions, and furthermore was location-dependent for optimum neutron flux measurement. A second monitor was placed in the Wisconsin Public Service Corporation's Kewaunee Nuclear Power Plant for a shorter period of time and similar information was obtained.

The final conclusion reported to the IAEA, was that the monitor would technically perform the functions that were required of it; however, it would not be completely reliable due to frequent mechanical failure. The IAEA was also advised that the only means to achieve the unattended monitoring of power reactors was to completely redesign the motor-gear train assembly of the monitor.

B. S. Carpenter

\section{Interferences in the Inductively Coupled Plasma (ICP) Technique}

In the application of the ICP technique to high-accuracy analysis of Standard Reference Materials (SRM's), interferences have been found which can degrade the quality of analytical results. These interferences can be traced to spectral and matrix-related interactions which must be characterized and corrected for in order to minimize their effect. The problem of spectral interference in the ICP technique is well known. 
Procedures for background and spectral overlap corrections have been established and are presently being employed in this laboratory. The first step is to expose a photographic plate with spectra of the sample and pure-element standards. Spectral lines are then chosen from considerations of sensitivity and absence of spectral overlap. The exit-slit carriage is mounted on the microdensitometer with the reference photographic plate, and slits are positioned in alignment with the chosen lines on the plate. Final alignment is accomplished by adjustment of a refractor plate in front of each slit.

A wavelength scan of each element is stored in the computer for output on the terminal. From plots of intensity-versus-wavelength for each element, the spectral peak position is checked and background correction points on either side of the peak are chosen. Spectral scanning and background sampling are accomplished by computer-controlled rotation of a refractor plate positioned behind the spectrometer entrance slit. In this manner, true net intensities for the spectral lines are calculated and either stored in the computer for calibration or displayed on the terminal.

Evidence has been found indicating differences in analytical response between pure-element standards and samples of complex matrices which are not due to spectral interference. These effects become significant when applying the ICP technique to high-accuracy analysis of SRM's. Chemical matrix effects in the plasma itself can cause up to a 15-percent relative enhancement or depression of the analyte element intensity depending on the spectral line and plasma operating conditions. Complete definition of the problem has involved examination of compromise conditions for simultaneous multielement analysis both from the point of view of sensitivity as well as matrix effect suppression.

One way to estimate a suspected matrix effect is to record net intensities for various elements at constant concentrations, with and without an excess $(3000 \mu \mathrm{g} / \mathrm{mL})$ of potassium present. The ratio of intensity with potassium to intensity without potassium can be plotted with respect to an instrument variable such as power, or observation height. The objective is to find operating conditions which result in a ratio close to 1.00 without significantly affecting the signal-to-background ratio. At present, no serious degradation in signal-to-background ratio has been caused by tuning the ICP for minimum matrix effect. However, the data include only five elements thus far, therefore the results must be considered to be preliminary. This data, as well as its extension to additional elements and matrices, will provide the information necessary to minimize analytical bias in simultaneous multielement analysis using the ICP technique.

R. L. Watters, Jr. 


\section{Atomic Weight of Silver}

A redetermination of the atomic weight of silver has been accomplished with an estimated accuracy of 1.4 parts in $10^{6}$ (based on a preliminary statistical evaluation of the data). The project was undertaken to serve the needs of the CODATA Task Group on Fundamental Constants. A high accuracy determination of the atomic weight of silver is of crucial importance because only with such a result in hand can full use be made of Bower and Davis' [1] sub-ppm determination of the electrochemical equivalent of silver to calculate a high accuracy value of the Faraday constant. The Bower-Davis Faraday value is presently based on the atomic weight of silver as determined 20 years ago at NBS [2]. This atomic weight, however, has an uncertainty twice as large as that obtained by Bower and Davis for the electrochemical equivalent of silver. By reducing the uncertainty in the atomic weight of silver by an order of magnitude, the Faraday constant could be improved by a factor of two. A reliable value of the Faraday will, in turn, permit the critical testing of the measured values of a number of constants including the Avogadro constant, the magnetic moment of the proton in units of the nuclear magneton, the ratio of the NBS as-maintained ampere to the absolute ampere, and the gyromagnetic moment of the proton. The new atomic weight data are to be turned over to representatives of the CODATA Task Group on Fundamental Constants who will be conducting a new least squares adjustment of the fundamental constants. The resulting recommended values are to be adopted for international use by the CODATA General Assembly at its 1981 meeting.

A high accuracy determination of the atomic weight of a polynuclidic element requires the combination of classical wet chemistry and high precision mass spectrometry. The methods developed for each must be precise to one part in $10^{4}$. For redetermination of the atomic weight of silver, a high precision chemical assay procedure was developed so that accurately known quantities of $107 \mathrm{Ag}$ and $109 \mathrm{Ag}$ separated isotopes could be mixed to produce standards for calibration of the mass spectrometer. The electromagnetically separated silver isotopes (approximately $1 \mathrm{~g}$ of each) were purified by a method in which silver was precipitated as silver chloride, dissolved in ammonium hydroxide and electrodeposited as metallic silver on a platinum dish. Each purified silver isotope was taken into solution with nitric acid and a portion was analyzed for impurities by isotope dilution spark source mass spectrometry. No impurity el ement was found at a concentration level greater than $10 \mathrm{ppm}$.

The concentration of each separated isotope solution was determined by a high precision-high accuracy method developed for natural silver solutions. The method is based on the gravimetric determination of the major portion of the silver (>99.7 percent) as silver iodide and the determination of the soluble residual silver by isotope dilution mass spectrometry. The uncertainty in the assay of these solutions at the 95 percent confidence 1 evel is 0.006 percent.

Eight calibration mixtures were prepared, by weight, from the separated isotope solutions. Six were within a few percent of the natural 
isotopic ratio and two were significantly altered, i.e. $1: 2$ and $2: 1$. The uncertainty in mixing the isotopes by weight is less than a few parts in $10^{5}$.

The relative isotopic compositions of the calibration mixes were determined by thermal-ionization mass spectrometry using a platinum single-filament silica gel technique. This technique, which involves loading sequential layers of silica gel, sample, and phosphoric acid onto the filament surface, has never been used before in high accuracy atomic weight determinations because the method of ionization enhancement is not well understood. However, in the case of silver, it proved to be the key to achieving levels of precision not possible with other modes of ionization. Thirty-two determinations of the relative isotopic composition of the silver standard, SRM 978a, were conducted with a standard deviation of 5.4 parts in $10^{5}$. It is hoped that the high level of precision obtained using the silica gel technique will encourage a more thorough investigation of this method of ionization enhancement.

Two complete sets of measurements of the calibration mixes and the silver standard (SRM 978a) were made by two different operators using different mass spectrometers. Each set consisted of four measurements of each calibration mix and thirty-two measurements of the reference standard. The samples were measured in a pattern alternating randomly selected mixes with the standard. Application of the correction factors obtained from the analysis of the calibration mixes to the relative isotopic composition of the silver standard allows the calculation of the absolute isotopic composition of that standard. The atomic weight of silver can then be calculated by summing the products of the absolute isotopic abundance of each isotope and its nuclidic mass.

Based upon preliminary calculations, the absolute isotopic abundance of the silver standard, SRM 978a, was found to be 1.0764 , yielding an atomic weight of 107.8682 . This determination of the atomic weight of silver will lead to a reduction of the uncertainty in the Faraday constant from $2.5 \mathrm{ppm} / \mathrm{S}$.D. to approximately $1.5 \mathrm{ppm}$, the original target value. This reduction in uncertainty will greatly enhance the usefulness of the silver Faraday in the CODATA least squares adjustment of the physical constants.

This determination of the atomic weight of silver will be published in the Journal of Research of the National Bureau of Standards.

$\underline{\text { References }}$

[1] Bower, V. E. and Davis, R. S., J. Res. Nat. Bur. Stand. (U.S.), 85 (1980).

[2] Shields, W. R., Craig, D. N., and Dibeler, V. H., J. Amer. Chem. Soc., 82, 5033 (1960).

L. P. Dunstan, J. W. Gramlich, T. J. Murphy, P. J. Paulsen 


\section{High Accuracy Mass Spectrometric Isotopic Analysis of Uranium}

Standards of known uranium isotopic composition are recognized as essential in the control and safeguarding of nuclear materials. NBS has traditionally provided Standard Reference Materials of known uranium isotopic composition to meet the needs of the nuclear community. Most of these SRM's are certified with an uncertainty of 0.1 percent. Trends indicate that in the near future this level of uncertainty will no longer meet the needs of regulations governing the nuclear industry because of the large quantities of materials involved. It is, therefore, essential that methodology be developed to allow measurement and certification of uranium isotopic compositions with approximately an order of magnitude smaller uncertainty.

The limiting factor in the accuracy and precision of mass spectrometric isotope ratio measurements for most elements is the ability to control and reproduce the rate of isotopic fractionation during the thermal ionization process. This rate of fractionation is influenced by the molecular weight of the species being volitalized from the sample filaments, as well as by the chemical and crystalographic form of the sample on the filament.

Standard procedures employed at NBS and most other laboratories performing uranium isotopic analysis involve conversion of the uranium to the oxide either as $\mathrm{UO}_{3}$ or $\mathrm{U}_{3} \mathrm{O}_{8}$ before analysis in the mass spectrometer. During the past year, the uranium oxide sample deposits were microscopically examined at various stages of the loading procedure. Since the uranium oxides are strongly colored and various crystal forms and degrees of hydration of $\mathrm{UO}_{3}$ show different coloration, it was easy to observe that reproducing the chemical and crystalographic species on the filament is extremely difficult. This difficulty is intensified when working with "real world" samples as opposed to high purity standards.

In an attempt to avoid the problems associated with the uncertain stoichiometry of the uranium oxide system, a new sample loading procedure was developed. A solution of uranium in nitric acid is evaporated at low temperature onto a rhenium filament to produce a deposit of uranyl nitrate hexahydrate. The filaments are then heated to approximately $60{ }^{\circ} \mathrm{C}$ to melt the hexahydrate, producing a uniform sample-filament coating. The hexahydrate is then converted to the dihydrate by heating at $100^{\circ} \mathrm{C}$ before loading into the mass spectrometer. At these low temperatures, formation of oxides is avoided. Use of this procedure over a six month period, has resulted in a precision for the analysis of the $235 \mathrm{U} / 238 \mathrm{U}$ ratio of 22 samples of SRM U-500, of 0.006 percent relative ( 1 std. dev.).

This procedure was recently used to certify the concentration and isotopic composition of SRM 995, a $233 \mathrm{U}$ spike solution. Eight ampoules of the SRM were calibrated by isotope dilution against both SRM 993, a $235 \mathrm{U}$ spike solution, and SRM 960, a uranium metal standard. In addition, SRM 993 was calibrated against SRM 960 , providing a direct correlation between the three SRM's. Statistical analysis of this work showed the uncertainty in the measured concentration of uranium in the SRM, including 
both random and possible systematic errors, to be less than 0.05 percent relative at the 99 percent confidence level. This procedure will also be used to certify the uranium isotopic composition in a series of three new uranium SRM's.

J. W. Gramlich, L. A. Machlan

14. Half-Life of $241 \mathrm{Pu}$

Accurate half-life values of the plutonium isotopes are needed to accurately certify and to keep current the isotopic composition of the NBS plutonium Standard Reference Materials. In addition, the accurate half-life values are needed in the fulfillment of the NBS commitment to provide essential standardization for nuclear safeguards and related measurements, especially the effort to develop SRM's for non-destructive assay techniques. An accurate value for the half-life of ${ }^{24} \mathrm{Pu}$ has been a particularly elusive parameter for more than a decade with measurements by alpha counting, calorimetry, and mass spectrometry giving values ranging from $13.8 \pm 0.3$ to $15.16 \pm 0.19$ years. Since the essence of a half-life determination is either the precise and/or accurate measurement of the number of atoms present at two points in time, or a series of isotopic ratio measurements as a function of time, the high precision (approaching 1 in $10^{4}$ ) capability of mass spectrometry is an attractive approach. The mass spectrometric measurements are divided into two parts, the chemical preparation of the sample and the isotopic ratio measurements using the mass spectrometer. The general sources of error recognized in the measurement are interference, isotopic fractionation, and analytical blank. The error due to analytical blank was found to be insignificant for the analysis of plutonium. The interference can be traced to isobars, background contributions from either organic or inorganic molecules and ion scatter or signal suppression from intense ion beams from other than plutonium. The major concern is for isobaric interference at mass 241 (americium), decay product of $241 \mathrm{Pu}$.

The chemical separation procedure, two passes through anion exchange columns in the nitrate form, were proven effective for the ${ }^{241}$ Am separation by gamma spectrometric analysis of a freshly separated plutonium sample and by mass spectrometric analysis of samples after repeated column separations. The other potential interference problems were reduced to an insignificant level by use of high-purity reagents, proper cleaning of the filament assembly and the ion exchange separation.

With ${ }^{241}$ Am removed from plutonium, the next greatest potential source of error is isotopic fractionation. The correction for isotopic fractionation for each of the six plutonium samples at time zero was determined by analyzing uranium SRM U500 under equivalent analytical conditions as plutonium. After the initial correction of the isotopic abundance ratios of each sample the ${ }^{239} \mathrm{Pu} / 240 \mathrm{Pu}$ ratio, after adjustment for radioactive decay, was used to determine the isotopic fractionation for all subsequent measurements. In addition, all analyses were made according to a strict analytical protocol to precisely reproduce the isotopic fractionation effect. 
The ${ }^{241} \mathrm{Pu}$ half-life results for independent decay curves for six different plutonium samples, including three SRM's varied from 14.32 to 14.36 years. The proposed half-1 ife value is $14.34 \pm 0.04$ years where the indicated uncertainty is the sum of the precision of the measurement and allowances for known sources of possible systematic error.

\section{E. L. Garner, L. A. Machian}

\section{High Precision Pulse-Counting}

Pulse-counting detection provides the highest sensitivity for measuring mass spectrometric ion beam currents and is the method of choice when ion beam currents are limited by sample size. For radioactive samples, the desirability of making measurements on minimum size samples is obvious to minimize safety risks to the analysts and contamination of the mass spectrometer. For this reason, a pulse-counting thermal ionization mass spectrometer has been put into operation and calibrated to provide measurements needed in the nuclear safeguards field. In the past year, this instrument has been used to study the resin bead sample loading technique, to certify plutonium and uranium standards, and to provide quality assurance for Faraday cup mass spectrometric measurements by the more accurate evaluation of loading blanks. Research has demonstrated that both uranium and plutonium can be analyzed, using nanogram samples, with precisions and accuracies less than 0.1 percent.

The resiri bead sample loading technique in which the sample is absorbed onto an ion exchange resin bead that is subsequently loaded directly onto the ionization filament of the mass spectrometer, has been singled out by the international safeguards community for its promising advantages of facilitating shipping as well as minimizing sample size and sample handling in the analysis of uranium and plutonium. The advantages of the technique are (1) the selective absorption of $U$ and $\mathrm{Pu}$ by the beads from the fission and actinide products in dissolver solutions, (2) the convenience of handling the nanogram quantities on beads including the ability to analyze beads directly in the mass spectrometer, and (4) the minimization of the radioactivity involved. The role of NBS in the resin bead program is to provide measurement assurance for the accurate isotopic analysis of uranium and plutonium. To fulfill this role, both chemical and mass spectrometric methodology for calibration and standardization have been investigated. Furthermore, a round robin has been organized which includes a majority of laboratories in the U.S. with the proven capability of making high sensitivity mass spectrometric measurements. The intent of the round robin is to generally investigate the reliability of the technique in terms of precision and accuracy achievable and to introduce this novel procedure to a broader range of laboratories.

A systems calibration, consisting of the determination of the $235 \mathrm{U} / 238 \mathrm{U}$ ratio in the well characterized SRM series $\mathrm{U}-050$ to $\mathrm{U}-930$, is the standard procedure in this laboratory for determining the limit of error in the isotopic measurement process. Systematic biases in this calibration are an indication of systematic errors which could result 
from lack of control of fractionation, discrimination, or inaccurate counting-loss correction. The results of a calibration in this laboratory using beads loaded with U-100, U-350, U-500, U-750, and U-900, respectively, exhibited a standard deviation of theoretical/experimental values of 0.045 percent. There were no significant discrimination or counting loss errors observed. Variability can be attributed to the differential isotopic fractionation behavior of individual beads.

In the preparation and evaluation of uranium loaded beads, it was found that the composition of the beads (nitrate-versus-chloride) and size of the beads drastically affect not only the loading efficiency of uranium from solution but also the ionization efficiency of the material absorbed on the bead in the mass spectrometer. Research is continuing on identification of the optimum parameters for preparing beads containing 1-3 nanograms of uranium that can be efficiently ionized in the mass spectrometer.

Research has been invested in the development of a procedure for the mass spectrometric determination of nanogram quantities of plutonium. The traditional procedure utilizing.a triple-filament loading technique, microgram quantities of material, and Faraday cup detection was deemed impractical for a large number of measurements in present laboratory facilities. This research culminated in a procedure in which 0.1 to 0.4 nanograms of plutonium were directly electroplated on a single rhenium filament and subsequently analyzed by the pulse counting mass spectrometer. This procedure was utilized in the characterization of a ${ }^{244} \mathrm{Pu}$ reference material by isotope dilution. In this characterization work, standards were analyzed with a standard deviation of 0.058 percent in the measurement of the $240 \mathrm{Pu} / 239 \mathrm{Pu}$ ratio. The concentration of the ${ }^{244} \mathrm{Pu}$ material was determined with a standard deviation of 0.06 percent. This assayed $244 \mathrm{Pu}$ reference material will allow both isotopic abundance and assay determinations to be made in a single isotope dilution mass spectrometric analysis, thus reducing the number of characterization measurements of unknown plutonium material by half.

J. D. Fassett, W. R. Kel1y

16. High Accuracy Method for Determining Sulfur in Iron Base Alloys

The knowledge and control of minor and trace concentrations of sulfur in iron and steel is exceedingly important for obtaining final products that possess desirable rather than deleterious properties. By far the most useful function of sulfur is its ability to improve the machinability of steels. For sulfur concentrations up to almost 0.3 percent, there is a concommitant improvement in the ease of machining with increasing amounts of sulfur. Paralleling this increase in machinability, however, is a decrease in strength and hardness, the loss of either of which may or may not be desirable. An area in which trace concentrations of sulfur definitely produce a detrimental effect in steels is stress corrosion. At concentrations above 0.01 percent, weldability is poor and the strengths of the welds are reduced appreciably. 
In terms of large tonnage production, the greatest effort to keep sulfur below 0.01 percent is made in the manufacture of ductile iron.

Because sulfur possesses this capability of imparting both positive and negative properties to iron and steel, the analytical need for standards whose sulfur content is known accurately is paramount. The present interest of CAC in the question of the accuracy of sulfur values in certified standards was initiated by two publications in the open literature which were brought to our attention $[1,2]$. Both publications came from the same Japanese laboratory and employed an isotope dilution gas mass spectrometer (IDGMS) procedure for analysis. Because of the widespread acceptance of IDMS as a definitive technique and the implication in these two papers that the certified sulfur values in several NBS SRM's might be as much as 20 percent high, the need to resolve this problem was considered particularly important.

After examining the experimental details of the two Japanese publications, it was concluded that the discrepancy between their IDGMS results and the certified values could possibly be accounted for by loss of sulfur before spike equilibration had occurred. This explanation certainly seemed feasible since, in the Japanese procedure, the samples were invariably dissolved before the spike was added.

To eliminate the possibility of losing sulfur through volatilization and at the same time to optimize conditions for sample and spike sulfur equilibration, we elected to perform all dissolutions in sealed, borosilicate tubes at elevated temperature and pressure. Since a gas mass spectrometer was not available, we further elected to use a spark source instrument for measurement of the ${ }^{34} \mathrm{~S} /{ }^{32} \mathrm{~S}$ ratio. To prepare the sample for spark source analysis, the sulfur was separated by reduction to $\mathrm{H}_{2} \mathrm{~S}$ and collected as $\mathrm{Ag}_{2} \mathrm{~S}$ by bubbling through a solution of $\mathrm{AgNO}_{3}$. The $\mathrm{Ag}_{2} \mathrm{~S}$ precipitate was centrifuged down, washed, mixed with high-purity gold powder and pressed, with repetitive folding, into the desired mounting form.

Using the sealed tube dissolution, the four NBS SRM's identified in the two Japanese publications (SRM's 32e, 33d, 72f, 133a) were reanalyzed for sulfur. In addition, five newer SRM's (361-365) were reanalyzed by the sealed tube procedure at NBS. The same SRM's were analyzed by the Japanese laboratory using their open-beaker procedures. Exchange and comparison of the data clearly indicate that whenever a discrepancy exists, the sulfur results obtained by open-beaker dissolution followed by ${ }^{34} \mathrm{~S}$ spiking are invariably lower than the sealed tube results. Using the open beaker dissolution followed by ${ }^{34} \mathrm{~S}$ spiking, we have been able to demonstrate that significantly lower results can be obtained. An even more disconcerting observation is that, even though the results are significantly lower, the precision is scarcely affected. For example, open beaker dissolution of NBS SRM 362 followed by ${ }^{34} \mathrm{~S}$ spiking yielded sulfur concentrations of 318,328 , and $320 \mathrm{ppm}$ for three separate sample analyses. Sealed tube dissolution, on the other hand, yielded sulfur values of 360,364 , and $357 \mathrm{ppm}$. The NB.S observations were transmitted to the Japanese laboratory together with a request to analyze SRM's 362 
and 364 by the sealed tube technique. These two SRM's were selected because the IDMS data from the two laboratories were most different for these. Using the sealed tube technique, the Japanese analyses of three separate samples of SRM's 362 and 364 yielded values of $358,357,360$, and $247,245,247 \mathrm{ppm}$, respectively. These results may be compared directly to the NBS sealed tube results obtained previously on SRM 362 (see above) and to the NBS results obtained on SRM 364, i.e., 247, 251, and $251 \mathrm{ppm}$ for three separate analyses.

The sealed tube dissolution technique in combination with IDSSMS has established that the NBS certified values for sulfur in those SRM's identified above are in close agreement with the IDMS measurements. The lower IDMS results reported earlier by the Japanese laboratory can best be accounted for by loss of sulfur before spike equilibration. Work is now underway to reanalyze additional SRM's in order to assess the accuracy of the certified sulfur values. Special attention is being given to establishing the accuracy of the sulfur values in the three major types of iron base alloys that are necessary for improving the accuracy of automated analyzers, namely low-alloy steels, high temperature alloys, and cast irons.

\section{References}

[1] Watanabe, K., Anal. Chim. Acta, 80, 117 (1975).

[2] Watanabe, K., Talanta, 26, 251 (1979).

R. W. Burke, G. M. Lambert, E. J. Maienthal, P. J. Paulsen

\section{Standard Reference Materials}

The analysis and certification of Standard Reference Materials (SRM's) are a major part of the total scientific effort of this Division, amounting to about 25 percent of the total budget with input from almost all of our scientists. The SRM's completed or started this year are shown in the following list. They include a wide variety of matrices (aqueous, botanical, zoological, fossil fuel, rock, metal, etc.) and were certified for concentration levels ranging from ultratrace to major.

A notable accomplishment was the certification of the inorganic electrolytes ( $\mathrm{Na}, \mathrm{Ca}, \mathrm{K}, \mathrm{Li}$, and $\mathrm{Cl}$ ) in SRM 909, Human Serum. This is the first human serum certified by NBS for inorganic constituents con tinually measured by clinical laboratories. It was certified using the definitive methods which were previously developed for the evaluation of the accuracy of clinical reference methods. A definitive method may be defined as one which has a valid and well defined theoretical foundation, has been experimentally evaluated so the reported results have neglible systematic errors, and has high levels of precision. 
Other significant efforts include the certification of a large number of trace and major constituents in an animal tissue (SRM, Bovine Liver), and a plant tissue (SRM, Citrus Leaves). Utilization of these SRM's will lead to improved accuracy of measurements used in health and environmental areas.

In the area of nuclear SRM's, a $233 \mathrm{U}$ spike and a ${ }^{244} \mathrm{Pu}$ spike have been certified for both isotopic composition and concentration. These SRM's will permit the determination of both properties in the same sample, reducing the sample's load by one half. Work was started on the certification of four new uranium isotopic standards for non-destructive analysis (NDA). These will be the first nuclear SRM's issued for NDA.

\section{Work Carried Out on Standard Reference Materials} by Division 551 During FY 80

SRM \#

$1620,1621 a, 1622$

966

995

$1577 a$

$1643 a$

1646

$76 a, 77 a, 78 a$

$1174,1175,1145$,

1146,1150

$\mathrm{C1} 287, \mathrm{Cl} 288, \mathrm{C1289}$, C1287a , C1288a, C1289a

$83 \mathrm{~d}$

$930 d$

1285,1286

1572

1084,1085

329

$1636 a, 1637 a, 1638 a$

$\mathrm{C} 1173, \mathrm{C1173a}$

1590

909
Title

Sulfur in Fuel 0 il

Pu 244 Spike

U 233 Spike

Bovine Liver

2.1

Trace El ements in $\mathrm{H}_{2} \mathrm{O}$

0.1

Estuarine Sediment

0.4

Zirconium in Burnt Refractories

0.1

White Cast Irons

0.1

High Alloy Steels

0.5

Arsenic Trioxide

0.1

Glass Filters for Spectrophotometry

0.3

Low Al loy Steels

0.2

Citrus Leaves

1.0

Wear Metals in 0 il

0.1

Uranium Non-destructive Analys is Standard

0.5

Zinc Concentrate

0.1

$\mathrm{Pb}$ in Reference Fuels

0.1

Cast Steels

0.1

Stabilized Wine

0.1

Human Serum
0.9 
Work Carried Out on Standard Reference Materials by Division 551 During FY 80 (continued)

$\underline{\text { SRM \# }}$

$890,891,892$

1270

1634

$1234,1235,1236$

$1237,1238.1239$

1269

2030

$723(a)$

$1224,14 \mathrm{f}$

$1261 \mathrm{a}, 1262 \mathrm{a}, 1263 \mathrm{a}$, $1264 a, 1265 a$

$136 \mathrm{~d}$

$691,691 a$

350

$1155 \mathrm{a}$

729,1225
Title

Al loy Cast Irons

$21 / 4 \mathrm{Cr}-1$ Mo Steel

Trace El ements in Fuel $0 i 1$

Zirconium Base Alloys

Line Pipe Steel

Glass Filters for Spectrophotometry

TRIS

Basic Open Hearth Steel

Low Al loy Steels

Potassium Dichromate

Reduce Iron Oxide

Benzoic Acid

Stainless Steel

Low Al loy Steel
Effort $M / Y$

0.9

0.3

0.9

0.1

0.3

0.1

0.2

0.3

0.3

0.1

0.2

0.1

0.1

0.3

Total

12.0 
C. Outputs and Interactions

(Inorganic Analytical Research Division)

1. Publications

Omenetto, N., Epstein, M. S., Bradshaw, J. D., Bayer, S., Horvath, J. J., and Winefordner, J. D., "Fluorescence Ratio of the Two D Sodium Lines in F1ames for D1 and D2 Excitation," J. Quant. Spectrosc. Radiat. Transfer, 22, 287 (1979).

Epstein, M. S., Nikdel, S., Omenetto, N., Reeves, R., Bradshaw, J. D., and Winefordner, J. D., "Inductively-Coupled Argon Plasma as an Excitation Source for Flame Atomic Fluorescence Spectrometry," Ana 1. Chem., 51, 2071 (1979).

Failey, M. P., Anderson, D. L., Zoller, W. H., Gordon, G. E., and Lindstrom, R. M., "Neutron-Capture Prompt $\gamma$-Ray Activation Analys is for Multi-Element Determination in Complex Samples, Anal. Chem., 51, 2209 (1979).

Alvarez, R. A., and Rains, T. C., "National Bureau of Standards Standard Reference Material for Quality Control of Nutrient Element Determinations in Food," Proc. of Nutrient Analysis Symposium, 93rd Annual Meeting of the Association of Official Analytical Chemists, Washington, DC, October 15-18, 1979, p. 86.

Carpenter, B. S., Pilione, L. J., Schroder, I. G., Roe, J. W., and Sanatini, S., "Evaluation of Reactor Track-Etch Power Monitor," ANS Transactions, Vol. 33, 21 (1979).

Mielenz, K. D., "Beam Splitter and Cell Window Errors in Fluorescence Spectrometry," App1. Optics, 18, 4134 (1979).

Brill, R. H., Yamasaki, K., Barnes, I. L., Rosman, K. J. R., and Diaz, M., "Lead Isotopes in Some Japanese and Chinese Glasses," Ars Orientalis, 11, 87 (1979).

Epstein, M. S., Omenetto, N., Nikdel, S., Bradshaw, J. D., and Winefordner, J. D., "Inductively-Coupled Argon Plasma as an Excitation Source for Flame Atomic Fluorescence Spectrometry," Anal. Chem., 52, 284 (1980).

Greenberg, R. R., "Simultaneous Determination of Mercury and Cadmi um in Biological Materials by Radiochemical Neutron Activation Analysis," Anal. Chem., 52, 676 (1980).

Lux, F., Zeisler, R., and Reher, J., "Aktivierungsanalytische Untersuchungen über die Probenmengenabhängigkeit der Streuung der Spurenelementgehaltswerte von Bleiweiss sowie über den cisalpinen Typ von Bleiweiss aus einem Rubens-Gemälde, "Radiochem. Radioana1. Letters, $\underline{42}, 341(1980)$. 
Lindstrom, R. M., "Measuring Trace Elements in Semiconductors: Methods and Pitfalls," Solar Cells, 1 (2), 117 (1980).

Koch, W. F., "The Value of the Faraday Via 4-Aminopyridine," in Atomic Masses and Fundamental Constants 6, J. A. Nolen, Jr. and W. Benenson, eds., (Plenum Press, NY, 1980), p. 167.

Epstein, M. S., Nikdel, S., Bradshaw, J. D., Kosinski, M. A., Bower, J. N., and Winefordner, J. D., "Atomic and Ionic Fluorescence Spectrometry with Pulsed Dye Laser Excitation in the InductivelyCoupled Plasma," Ana 1. Chim. Acta, 113, 221 (1980).

Epstein, M. S., Bayer, S., Bradshaw, J. D., Voigtman, E., and Winefordner, J. D., "Application of Laser-Excited Atomic Fluorescence Spectrometry to the Determination of Iron," Spectrochim. Acta, 35B, 233 (1980).

Epstein, M. S., Bradshaw, J. D., Bayer, S., Bower, J., Voigtman, E., and Winefordner, J. D., "Application of Laser-Excited Atomic Fluorescence Spectrometry to the Determination of Nickel and Tin," App1. Spectrosc., 34, 372 (1980).

Epstein, M. S., and Winefordner, J. D., "Precision and Linearity of Determinations at High Concentrations in Atomic Absorption Spectrometry with Horizontal Rotation of the Burner, "Talanta, 27, 177 (1980).

Christian, G. D., and Epstein, M. S., "Atomic Absorption Spectroscopy," American Chemical Society Audio Course, American Chemical Society, Washington, DC (1980).

Deardorff, E. R., Rains, T. C., and Koch, W. F., "Simulated Precipitation Reference Materia1s, III," NBSIR 79-1953, Apri1 1980.

Carpenter, B. S., and Jones, F. E., eds., "Measurements and Standards for Nuclear Materials Safeguards," NBSIR 80-2033, May 1980.

Velapoldi, R. A., Paule, R. C., Schaffer, R., Mandel, J., Machlan, L. A., Garner, E. L., and Rains, T. C., "Standard Reference Materials: A Reference Method for the Determination of Lithium in Serum," NBS Spec. Pub1. 260-69, U.S. Department of Commerce, 1980, p. 1.

Dunstan, L. P., Gramlich, J. W., Barnes, I. L., and Purdy, W. C., "Absolute Isotopic Abundance and the Atomic Weight of a Reference Sample of Thal1ium," J. Res. Nat. Bur. Stand. (U.S.) 85, 1 (1980).

Stacey, J. S., Delevaux, M. H., Gramlich, J. W., Doe, B. R., and Roberts, R. J., "A Lead Isotope Study of Mineralization in the Arabian Shield," U.S. Geological Survey Saudi Arabian Mission Technical Record 8, 1980. 
Canada, T. R., and Carpenter, B. S., eds., "Measurement Technology for Safeguards and Materials Control," NBS Spec. Pub1. 582 (1980).

Mitche11, J. W., Riley, Jr., J. E., and Carpenter, B. S., "Major Elemental Boron Determination by the Nuclear Track Technique," ANS Transactions, Vo1. 34, 143 (1980).

Kelly, W. R., and Wasserburg, G. J., Letter in response to article "Did the Solar System Start with a Bang?", Sky and Telescope, $59,14(1980)$.

Fassett, J. D., Kelly, W. R., Machlan, L. A., and Moore, L. J., "Mass Spectrometric Isotope Metrology of Uranium on Resin Beads," in Radioelement Analys is Progress and Problems, W. S. Lyon, ed., Ann Arbor Science, p. 357, 1980.

Kaiser, T., Kelly, W. R., and Wasserburg, G. J., "Isotopically Anomalous Ag in Two Iron Meteorites," XI Lunar and Planetary Science Conference Abstracts, Vol. 2, The Lunar and Planetary Institute, Houston, TX, p. 527 (1980).

Kaiser, T., Kelly, W. R., and Wasserburg, G. J., "Isotopically Anomalous Silver in the Santa Clara and Piñon Iron Meteorites," Geophys. Res. Letters, 7,271 (1980).

Lindstrom, R. M., and Fleming, R. F., "Accuracy in Activation Analysis: Count Rate Effects," to be published in Proc. of 4th International Conference on Nuclear Methods in Environmental and Energy Research (Univ. of Missouri, Columbia), 1980.

Mavrodineanu, R., and Baldwin, J. R., "Metal-On-Quartz Filters as a Standard Reference Material for Spectrophotometry--SRM 2031 ," NBS Spec. Publ. 260-68 (1980).

Harrison, S. H., Maienthal, E. J., Gills, T. E., and Zeisler, R., "Protocol for the Collection, Storage, and Analys is of Human Organs the Pilot Bank Project of the National Environmental Specimen Bank," in Trace Substances in Enviromental Health, XIII, D. P. Hemphi11, ed., U. of Missouri, Columbia, June 1980, in press.

Paulsen, P. J., Burke, R. W., Maientha 1, E. J., and Lambert, G. M., "Isotope Dilution Spark Source Mass Spectrometric Determination of Sulfur in Some Current NBS-SRMs (Fe-based Alloys), Proc. ASTM Symposium on New Analytical Techniques for Trace Constituents of Metal-Bearing Systems, Chicago, IL, June 1980, in press.

Carpenter, B. S., and Pilione, L. J., "Lithium and Lithium-6 Analysis in Solutions by the Nuclear Track Technique," Anal. Chem, in press. 
Moore, L. J., Heald, E. F., and Barnes, I. L., "Thermodynamic and Empirical Studies of Fractionation in the Thermal Ionization Multiple Filament Source," Proc. of the 8th International Conference on Mass Spectrometry, Oslo, Norway, in press.

Harrison, S. H., Zeisler, R., and Wise, S. A., "Pilot Program for the National Environmental Specimen Bank--Phase I," to be published as an NBS Tech. Note.

Kingston, H. M., and Pella, P. A., "X-Ray Spectrometric Analys is of Trace Metals in Environmental and Biological Samples Using Exchange Resin-Loaded Filters," submitted to Analytical Chemistry, July 1980.

Horvath, J., Bower, J., Bradshaw, J., Epstein, M. S., and Winefordner, J. D. " "Comparison of Nebulizer-Burner Systems for Laser-Excited Flame Atomic Fluorescence Spectrometry," submitted to Anal. Chem.

Kaiser, T., Kelly, W. R., and Wasserburg, G. J., "Hoba and Tlacotepc: Two New Meteorites with Isotopically Anomalous Ag," submitted to 43rd Meteoritical Society Meeting, San Diego, CA, Sept. 2-6, 1980.

Zeisler, R., Greenberg, R. R., and Gills, T. E., "Standard Reference Water and Its Multi-Element Assay by Nuclear Activation Techniques," submitted to J. Environ. Anal. Chem.

Wynn, T., Clardy, P., Bower, J., Bradshaw, J., Epstein, M., and Winefordner, J. D. , "Wavelength-Modulated Continuum-Source Atomic Fluorescence Spectrometer for Wear Metal Analysis in Jet Engine 0ils Using Electrothermal Atomization," submitted to Anal. Chim. Acta.

2. Talks

Fassett, J. D., Kelly, W. R., Machlan, L. A., and Moore, L. J., "Mass Spectrometric Isotope Metrology of Uranium on Resin Beads," Twenty-Third Conference on Analytical Chemistry in Energy Technology, Gatlinburg, TN, October 10, 1979.

Rains, T. C., "NBS Standard Reference Materials for Quality Control of of Nutrient Element Determination in Foods, "93rd Annual Meeting of Official Analytical Chemists, Washington, DC, October 15, 1979.

Harrison, S. H., "The Sampling and Storage Protocols for the Collection of Human Livers," Joint U.S./German Bilateral Meeting on Specimen Banking, National Bureau of Standards, Washington, DC, November 15, 1979.

Zeisler, R. L., "Development of an Analytical System to be Applied in the NESB Program," Joint U.S./German Bilateral Meeting on Specimen Banking, National Bureau of Standards, Washington, DC, November 15, 1979. 
Greenberg, R. R., "Simultaneous Determination of Arsenic, Antimony, Cadmium, Chromium, Copper, Molybdenum, Selenium, and Silver in Tissue Samples by Radiochemical Neutron Activation Analysis, Joint U.S./German Bilateral Meeting on Specimen Banking, National Bureau of Standards, Washington, DC, November 16, 1979.

Maientha1, E. J., "Voltammetry," Joint U.S./German Bilateral Meeting on Specimen Banking, National Bureau of Standards, Washington, DC, November 16, 1979.

Gramlich, J. W., "Lead Isotopic Analysis of Glass," Centennial Meeting of the Archaeological Institute of America, Boston, MA, December 28, 1979.

Watters, Jr., R. L., "Sampling and Analysis Strategies for Plasma Spectrochemical Analysis," International Winter Conference 1980-Developments in Atomic Plasma Spectrochemical Analysis, San Juan, PR, January 10, 1980.

Burke, R. W., "NBS Studies on ASTM Special Fly Ash," ASTM Winter Meeting, Ft. Lauderdale, FL, January 30, 1980.

Kingston, H. M., "A Prospective of Ultra Trace Elemental Analysis in Chesapeake Bay Water with a Discussion of Clean Laboratory and Field Sampling Technology, National Oceanic and Atmospheric Administration, Rockville, MD, January 31, 1980.

Rains, T. C., "Atomic Absorption Spectrometry," ACS Short Course, Pittsburgh Conference, Atlantic City, NJ, March 8, 1980.

Zeisler, R. L., "The National Environmental Specimen Bank--EPA/NBS Pilot Program," Center for Trace Characterization Seminar, Texas A\&M University, College Station, TX, March 15, 1980.

Kaiser, T., Kelly, W. R., and Wasserburg, G. J., "Isotopically Anomalous Silver in Two Iron Meteorites," XI Lunar and Planetary Science Conference, Houston, TX, March 21, 1980.

Kingston, H. M., "Analytical Chemical Techniques as Applied to Analysis of Water in the Chesapeake Bay," Student Affiliation of the American Chemical Society, Thiel College, Greenville, PA, March 27, 1980.

Lindstrom, R. M., "Accuracy in Activation Analysis: Beyond Net Areas," Fourth International Conference on Nuclear Methods in Environmental and Energy Research, Columbia, MO, Apri1 14, 1980.

Rains, T. C., "The State-of-the-Art of Atomic Absorption Spectrometry with Electrothermal Atomization," St. Louis Section of the Society for Applied Spectroscopy, St. Louis, M0, Apri1 17, 1980.

Zeisler, R. L., "The U.S. National Environmental Specimen Bank Program," Institut für Radiochemie Seminar, Technische Universität München, Munich, F. R. Germany, May 23, 1980. 
Rains, T. C., "State-of-the-Art--Ultra Metal Analysis in AAS," Mayo Clinic, Rochester, MN, May 30, 1980.

Gramlich, J. W., "Sample Mounting Techniques for High Accuracy Uranium Isotopic Analysis," 29th Annual Conference on Mass Spectrometry and Allied Topics, New York, NY, June 4, 1980.

Kingston, H. M., "Chesapeake Bay Trace and Toxic El emental Measurement; What.Do We Have and Where is the Value," NBS Colloquium on Mathematical Modeling in Science and Engineering, National Bureau of Standards, Washington, DC, June 11, 1980.

Paulsen, P. J., "Isotope Dilution Spark Source Mass Spectrometric Determination of Sulfur in Some Current NBS-SRMs (Fe-Based Alloys)," ASTM Committee E-03 Symposium on "New Analytical Techniques for Trace Constituents of Metallic and Metal-Bearing Ores," Chicago, IL, June $24,1980$.

Kelly, W. R., "Palladium-107 Isotopic Heterogeneity in the Early Solar System," Department of Terrestrial Magnetism, Carnegie Institute of Washington, Washington, DC, June 25, 1980.

Maienthal, E. J., "Voltammetric Analysis of Things as They Are," Harvey Diehl Symposium, Second Chemical Congress of the North American Continent, American Chemical Society Meeting, Las Vegas, NV, August 29, 1980.

Gramlich, J. W., "Recent Advances in High Accuracy Thermal Ionization Mass Spectrometry," Symposium on Scientific Instrumentation, University of Mexico, Mexico City, Mexico, September 2, 1980.

Greenberg, R. R., Kingston, H. M., and Beary, E. S., "Analys is of Estuarine Water and Associated Suspended Solids," VII Federation of Analytical Chemistry and Spectroscopy Societies, Philadelphia, PA, September 29, 1980.

\section{Committee Assignments}

I. Lynus Barnes

Member, Department of Health, Education, and Welfare, The Chemistry Task Force of the National Shellfish Sanitation Program Washington Editorial Review Board International Union of Pure and Applied Chemistry, Commission on Atomic Weights

Secretary, IUPAC, Commission on Atomic Weights, Subcommittee for Assessment of Isotopic Abundances

Robert W. Burke

Member, ASTM E-03 Chemical Analysis of Metals 
B. Stephen Carpenter

Member, American Nuclear Society Board of Directors American Nuclear Society 16, Isotopes and Radiation American Nuclear Society, Neutron Activation Analysis Institute for Nuclear Material Management, Workgroup 9.3, Standards for Nondestructive Assay

Barry I. Diamondstone

Member, ASTM D-34.02.01, Section on Batch Extractions ASTM D-19.2, Section on Hazardous Wastes in Water ASTM E-03, Chemical Analysis of Metals

Michael S. Epstein

Secretary, Baltimore-Washington Section of the Society for Applied Spectroscopy

Member, ASTM D-19.07.04, Section on Chemical Characterization of Fluvial Sediment ASTM D-19, Water

Registrations Chairman, VII Annual Meeting of the Federation of Analytical Chemistry and Spectroscopy Societies

Ronald F. Fleming

Member, Program Committee, Fifth Symposium on X- and Gamma-Ray Sources and Applications

Thomas E. Gills

Member, ASTM C-26, Committee on Nuclear Fuel Cycle

Howard M. Kingston

Member, NES Nuclear Waste Study Group

William F. Koch

Member, ASTM D-19, Water ASTM D-19.02, General Specifications and Technical Resources ASTM D-19.05, Inorganic Constituents in Water

Lawrence A. Machlan

Member, ASTM C-26, Nuclear Fuel Cycles ASTM C-26.01, Editorial and Terminology ASTM C-26.05, Test Methods

E. June Maienthal

Member, ASTM D-19, Water

ASTM D-19.05, Inorganic Constituents in We.ter

ASTM D-19.06, Methods for Analysis for Organic Substances

in Water

ASTM. D-19.07, Methods of Testing of Water-Reformed Deposits and Properties of Weter ASTM D-19.07.04.03, Trace Metals and Sediments ASTM D-19.11, Methods for the Analysis of Water for Power Generation and Process Uses 
ASTM E-03, Chemical Analysis of Metals

ASTM E-03.01, Ferrous Metals

ASTM E-03.02, General Analytical Practices

ASTM E-03.05, Nonferrous Metals

Standard Methods for Examination of Water and Waste Water

Joint Task Group for Polarographic Methods

George Marinenko

Member, ASTM D-19, Water

ASTM D-19.02, Specifications

ASTM D-19.05, Incrganic Constituents in Water

ASTM D-19.09, Saline Water

ASTM D-19.05.03.06, Ozone

Program Chairman, National Capital Section of the Electrochemical Society

Jerry D. Messman

Assistant Registrations Chairman, VII Annual Meeting of the Federation of Analytical Chemistry and Spectroscopy Societies

Coordinator, Science Fair Program for the Baltimore-Washington Section of the Society for Applied Spectroscopy

Klaus D. Mielenz

Member, TC-3, Luminescence Subcommittee, International Commission

on Illumination

TC-2.3, Materials Luminescence

ASTM E-13, Molecular Spectroscopy

ASTM E-13.06, Molecular Luminescence

John R. Moody

Member, ASTM D-19, Wäter

ASTM D-19.05, Inorganic Constituents in Water

Delegate, Middle Atlantic Regional Meeting, Chemical Society of Washington

Thomas J. Murphy

Chairman, Audit Committee, Chemical Society of Washington

Councilor, Chemical Society of Washington

Member, American Chemical Society Committee on Analytical Reagents American Chemical Society Committee on Membership Affairs American Chemical Society Admissions Committee International Union of Pure and Applied Chemistry (IUPAC), Commission on Atomic Weights and Isotopic Abundances

Member, International Union of Pure and Applied Chemistry (IUPAC), Subcommittee for the Assessment of Isotopic Abundances, Commission on Atomic Weights and Isotopic Abundances 
John A. Norris

Secretary, ASTM E-02, Emission Spectroscopy

Lecturer, Annual Short Course, "Modern Industrial Spectroscopy," Arizona State University, Tempe, AZ

Lura J. Powel1

Chairman, Budget and Finance Committee, Chemical Society of Washington

Manager, Chemical Society of Washington

Financial Chairman, 1981 Middle Atlantic Regional Meeting, American Chemical Society

Member, Publicity Committee, VII Annual Meeting of the Federation of Analytical Chemistry and Spectroscopy Societies

Chairman, Committee on Isotope Ratio Measurements, American Society for Mass Spectrometry

Theodore C. Rains

Chairman, International Conference Committee of the Society for Applied Spectroscopy

Member, Proposal Evaluation Committee for National Science Foundation AOAC Committee on Automated Methods

Nominating Committee for the Society for Applied Spectroscopy Editorial Board of Annual Reports on Analytical Atomic Spectroscopy (The Chemical Society of London) and the Canadian Journal of Spectroscopy

ASTM E-02.10, Non-Metal1ic Materials

ASTM E-02.13, Nomenclature

ASTM D-01, Paint and Related Coatings

ASTM D-19, Water

Co-chairman, Program, VII Annual Meeting of the Federation of Analytical Chemistry and Spectroscopy Societies

Director, Professional Development Program for the BaltimoreWashington Section of the Society for Applied Spectroscopy

Column Editor, Journal of Applied Spectroscopy

Professor-in-charge, ACS Short Course on "Atomic Absorption Spectroscopy"

Task Force Leader for Project Scientific Instrumentation Information Network and Curricula (SIING) on Atomic Absorption Spectroscopy

Robert L. Watters, Jr.

Secretary, ICP Detection Limits Committee

National Correspondent and Reviewer Designate, ICP Information Newsletter 


\section{Organic Analytical Research Division}

Harry S. Hertz, Chief

Dennis J. Reeder, Deputy Chief

\section{A. Division Overview}

The activities of the Organic Analytical Research Division encompass a wide variety of research capabilities and measurement procedures. Generic research in organic analytical chemistry has resulted in the development of new analytical methods for the detection, characterization, and quantitation of trace amounts of organic compounds found in complex matrices. The analytical capabilities of the Division are applied to measurement problems in environmental, health/clinical, and energy-related areas. Results of the work performed by the Division are utilized by other federal agencies, public and private institutions, and by research programs at NBS.

The year just completed has been very productive. The accomplishments have been numerous and the products of research exciting. We have summarized some of these accomplishments below and many are presented in more detail in the individual technical accomplishments which follow. In reading the group overviews and the technical accomplishments, there are several points of particular note. The division is organized into groups by areas of specialization: organic spectroscopy, gas chromatography, liquid chromatography, mass spectrometry, bioanalytical techniques, and organic electrochemistry. Projects, however, usually cross group lines and, in the past year, we have witnessed an extensive number of successful intergroup (and interdivision) task forces being formed and succeeding on difficult problems. The groups have begun research along the lines presented in last year's five-year research plans. The organic electrochemistry competence building group has successfully started many of their projects and initiated collaborative research efforts with other groups on several projects.

Three yery significant Standard Reference Materials were completed this year: SRM 1644, Generator Columns for Polynuclear Aromatic Hydrocarbons in Water; SRM 1580, Shale 0il; and SRM 909, Human Serum. A patent was awarded on the NBS 1 iquid chromatograph-mass spectrometer (LC-MS) interface and considerable additional research has been performed on LC-MS this past year. The first NBS "definitive" method for the determination of an organic chemical in serum (cholesterol) was published in Clinical Chemistry and four additional definitive methods either are completed or well along in their development (glucose, uric acid, urea, and creatinine). In a study of the retention behavior of polynuclear aromatic hydrocarbons on high-performance liquid chromatographic (HPLC) bonded phases, it was found that the retention could be predicted on the basis of the length to breadth ratio of the molecules. Research in the area of multi-dimensional gas chromatography has expanded and the certification of 5,6-benzquinoline in the shale oil SRM was achieved through use of this new capability. Installation of the 400 MHz nuclear magnetic resonance (NMR) spectrometer has been completed and the instrument already has been used for several research problems, including the high-accuracy quantitative analysis of trace constituents in several existing and proposed SRM's. 
During the past year, two new NRC post-doctoral fellows have joined our division. One fellow will be investigating electrode reaction mechanisms using surface enhanced Raman spectroscopy to provide molecular spectra of adsorbed reactants, intermediates, and products. The second NRC fellow will be investigating synthetic routes to and the NMR spectra of uniquely labeled ${ }^{15} \mathrm{~N}$ aminoglycosides, nucleotides, and nucleosides.

In the year ahead, we look forward to increasing our emphas is on more basic research programs in spectroscopy by development of total luminescence techniques for mixture analysis, in the use of enzymes in analytical chemistry, in gas chromatography by continued development of multicolumn techniques, in liquid chromatography by investigating post-column reaction systems, in mass spectrometry by investigating ionization mechanisms and ion structures, and in electrochemistry by development of chemically modified electrodes as well as research in spectroelectrochemistry. With the completion of several major SRM's and a decrease in some of our programmatic commitments, we eagerly are anticipating the opportunity to expand our efforts at the state-of-the-art in organic analytical chemistry.

\section{Organic Spectroscopy Group: Overview}

The Organic Spectroscopy Group has continued its general responsibility for: (1) research on determination of the fundamental molecular and system parameters that affect measurement accuracy; (2) qualitative and quantitative analyses of compounds and mixtures; and (3) characterization and certification of SRM's using nuclear magnetic resonance (NMR), polarimetry, and fluorescence techniques.

Work this past year in the area of optical spectroscopy has dealt mainly with research on development of "on-stream" analysis of aqueous solutions of polynuclear aromatic hydrocarbons (PAH's) at the ppb level, developing capabilities for on-stream total fluorescence spectroscopy, proving the feasibility of using laser polarimetric techniques for optical rotatory dispersion, and verifying the efficacy of derived equations for the optical rotation of sucrose and quartz at different wavelengths. Additionally, certification of SRM 1644 (Generator Columns) for production of known aqueous standards of PAH's for use in the environmental and energy effluent areas, and of SRM 17c (Sucrose) using the high-accuracy polarimeter has been completed. Measurements on $\mu \mathrm{m}$-sized fluorescence glass beads as potential standards for microspectrofluorimetry have also been completed. Expansion of research in the analytical application of FT-IR and development of new total luminescence techniques for the analysis of mixtures is anticipated during the next year.

Much of the effort this year in NMR spectroscopy has been devoted to the installation and testing of two, high-field superconducting NMR spectrometers, which together form the high-field NMR facility of the National Measurement Laboratory. Members of the group have had the major responsibility for operation of the $400 \mathrm{MHz}$ spectrometer, while personnel of the Chemical Corrosion and Stability and Polymer Divisions have had the main responsibility for a new $200 \mathrm{MHz}$ instrument. 
In spite of a number of quality control and testing problems, the new $400 \mathrm{MHz}$ spectrometer has generally exceeded specifications for proton, carbon-13, and nitrogen-15 sensitivity and resolution by about 30 percent. During this year, proton NMR spectroscopy at $400 \mathrm{MHz}$ has been applied to high-sensitivity, high-accuracy, quantitative analysis of trace impurities in sodium pyruvate and to studies of the stability in aqueous solution of isotopically-labeled marker compounds for pollutant analysis. This powerful technique has allowed complete resolution for the first time of the proton NMR spectra of methyl 4,6-0-benzylidene and 2,6-anhydro- $\alpha$-D-altropyranosides, compounds which are of interest to us as intermediates for the synthes is of nitrogen-15 labeled amino sugars in which the nitrogen isotope has a defined stereochemical orientation.

Another program in nitrogen-15 NMR spectroscopy uses materials in which the nitrogen-15 isotope is either enriched or at natural abundance. Included in this program is the exploration of nitrogen-15 NMR for characterization of antibiotics and other biochemically important nitrogenous materials.

Carbon-13 NMR at high field has permitted increased detectability and accuracy of quantitation for aromatic components of recycled oil and methods have been investigated for increasing this accuracy further by removal of variable nuclear Overhauser effects. The accuracy of quantitation is also influenced by carbon-13 spin-lattice relaxation times and basic information on these parameters of the recycled oils is being generated by measurements at both high and low field strengths.

In the future, we plan to refine our competence in homonuclear, twodimensional NMR techniques and extend these techniques to measurement of heteronuclear interactions between protons, and carbon-13 and nitrogen-15 nuclei. Assuming the availability of certain key accessories for the $400 \mathrm{MHz}$ spectrometer, we also plan to investigate the potential of nuclear cross-polarization techniques in liquids for sensitivity enhancement in NMR analysis. The multi-nuclear capability of the instrument will also be extended by installation of one or more broad-band probes. This capability will allow NMR analysis at high field of magnetically active nuclei other than protons, carbon-13, nitrogen-15, and phosphorus-31.

Bruce Coxon, Group Leader; Robert E. Botto, Robert M. Davidson, Diane K. Hancock

\section{Bioanalytical Techniques Group: Overview}

The publication of a first NBS definitive method for the determination of an organic chemical in serum entitled "Total Serum Cholesterol by Isotope Dilution/Mass Spectrometry," appeared in Clinical Chemistry [1]. This paper is the forerunner of a series on NBS research on organic definitive methods. The newest organic ID/MS method, for serum creatinine, is now undergoing evaluation as a definitive method. The definitive and reference methods for chloride in serum and lithium in serum were published as NBS-260 reports $[2,3]$. These reports describe the ID/MS methods and the respective electrometric and atomic absorption methods used as reference methods for these 
electrolytes, and provide the data and a statistical summary of the results obtained on use of the two reference methods in groups of clinical laboratories that prove the methods' applicability.

The definitive methods developed thus far are now being applied to certifying the values of a number of analytes in a human serum that is to be issued in 1980 as an SRM. The organic analyses are carried out by the joint effort of the Bioanalytical Techniques and Mass Spectrometry Groups of this Division and the inorganic analyses are performed by the Inorganic Analytical Research Division. The enzyme activity levels in the human serum SRM are also being measured; the data is being produced in a group effort in a large number of clinical laboratories. We are participating with measurements of lactate dehydrogenase activity.

We have completed work on the certification of crystalline 4-nitrophenol as an SRM. It is issued for use as a spectrophotometry standard for assays of alkaline phosphatase activity. Our group determined the purity of the 4-nitrophenol, and the Inorganic Analytical Research Division cooperated by performing the spectrophotometric measurements.

A study is underway to isolate and identify the low molecular weight organic chemical substances which, under physiological conditions, leach from the polymeric materials that are employed with medical devices, including the silicone polymers used in surgically implanted prostheses. We are also undertaking to establish the effect that the substances leached from the silicone polymeric material have on the activity of the enzyme prolyl hydroxylase. The formation of masses of neoplastic tissue around each implant are thought to be related to the activity of that enzyme. Methods development for the purification of the enzyme for the purpose of improving the activity assay is also part of the study.

Another project in our continuing research on the use of enzymes in organic analytical chemistry involves the preparation of pyridoxamine pyruvate transaminase and related enzymes from a bacterial source. The enzymes are being purified for the purpose of developing analytical procedures for the measurement of pyridoxine and pyridoxamine, as parts of a Vitamin $B_{6}$ assay.

A 1 iterature survey and a research plan have been prepared on the development needed for personal monitoring devices for toxic organic compounds in the ambient atmosphere.

A fluoroimmunoassay for 2,4-dinitrophenol in water has been developed and is now being refined to increase its sensitivity. This assay utilizes specific rabbit-antibody, immunoglobulin molecules that are attached covalently to solid-phase polymeric beads by coupling with a carbodiimide reagent. 2,4-Dinitrophenol in the test system competes for the specific antibody and thus reduces the amount of fluorescently labeled tracer bound by the antibodies. Fluorescence is measured by photon counting of the bound fractions after they have been separated from the unbound reagents. This study is intended as a model for the potential use of rapid, small molecule, immunoassay procedures in the analysis of aqueous environmental samples. 
The need to provide the base for ensuring accuracy in the analysis of biological samples (in particular, analytes in serum and other body fluids) prompted our development of definitive methods for a number of organic analytes. Many such methods are needed, and we plan to continue our development of these methods in 1981. Current high-priority analytes include creatinine, digoxin, and thyroxine.

Biological reagents can provide a novel means for determining analytes in non-biological samples. In 1981, we expect to continue to explore such possibilities through further development of immunochemical methods and through assays employing enzymes.

\section{References}

[1] Cohen, A., Hertz, H. S., Mandel, J., Paule, R. C., Schaffer, R., Sniegoski, L. T., Sun, T., Welch, M. J., and White V, E., Total Serum Cholesterol by Isotope Dilution/Mass Spectrometry: A Candidate Definitive Method, Clin. Chem. 26 (7), 854-860 (1980).

[2] Velapoldi, R. A., Paule, R. C., Schaffer, R., Mandel, J., Murphy, T. J., and Gramlich, J. W., A Reference Method for the Determination of Chloride in Serum, NBS Special Publication 260-67 (1979).

[3] Velapoldi, R. A., Paule, R. C., Schaffer, R., Mandel, J., Machlan, L. A., Garner, E. L., and Rains, T. C., A Reference Method for the Determination of Lithium in Serum, NBS Special Publication 260-69 (1980).

Robert Schaffer, Group Leader; Alex Cohen, Alexander J. Fatiadi, Barbara F. Howel1, Dennis J. Reeder, Pieter VanDerLijn

\section{Electrochemistry Group: Overview}

The recently initiated (competence building) program in organic electrochemistry is progressing well in the directions originally proposed for this research effort, i.e., spectroelectrochemistry, automation, and chemical modification of electrodes. During the second year of this program, the organic electrochemistry group has reached full staffing. Most of the major pieces of essential instrumentation have been acquired, although a variety of important ancillary instruments are still needed to permit maximum effectiveness in achieving our planned research goals.

In the area of spectroelectrochemistry, the setting up of the vidicon optical multichannel analyzer system has been completed, and evaluation of the system using the well-characterized compound, o-tolidine, has demonstrated that the performance of the system is excellent for studies of this type. Using an optically transparent thin-layer cell with a gold minigrid working electrode, it was possible to obtain the formal potential and stoichiometry of the o-tolidine from the spectroelectrochemical data. The values obtained showed excellent agreement with literature data obtained 
by rapid scanning spectrometers. However, the vidicon system is more sensitive than scanning spectrometers which will become important in future research into transient redox reactions.

The computer-controlled electrochemical system (CCES) has been constructed and most of the necessary software written to permit a diversity of excitation waveforms, data manipulations, and graphic displays to be performed. A commercial potentiostat has been incorporated into the CCES in order to evaluate its performance. To improve the operational characteristics of the CCES, the analog/digital data conversion system was located external to the computer mainframe and a direct memory access was designed and is being constructed. A specially designed cell for studies using rapidly changing excitation signals has also been constructed and is in the process of evaluation.

Research into the development of improved electrochemical detectors for 1 iquid chromatography has resulted in a high-sensitivity, dualelectrode cell which will minimize noise and drift problems caused by traces of residual oxygen. A comparison of the dual-electrode with a conventional electrochemical detector for selected organic and organometallic analytes demonstrated the superior sensitivity of the new approach. Further studies are in progress to evaluate this detector for improved selectivity by using modulated excitation waveforms and phase-sensitive detection.

Research into the chemical modification of electrodes is underway in two studies. In one, several nickel(II) Schiff-base complexes have been synthesized and will be attached to a platinum electrode via a polyphenylene oxide film. It is expected that these bound complexes will undergo electrocatalysis with aliphatic and aromatic halogenated hydrocarbons. If this type of modified electrode is stable, it will be developed as a selective detector for liquid chromatography.

The other type of modified electrode is a semiconductor, $n$-type $\mathrm{TiO}_{2}$, which when irradiated by UV light is capable of oxidizing carboxylic acids at very low potentials thereby avoiding interfering redox processes. By means of this photo-Kolbe reaction, it is planned to develop a photoelectrochemical high-performance liquid chromatography detector for organic acids. A specific application of this system will be the certification of valproic acid in serum as an SRM.

The potassium salt of croconate violet was investigated using cyclic voltammetry and spectroelectrochemistry. From these experiments, plus digital simulation of the data, it was possible to calculate thermodynamic and kinetic parameters and to demonstrate that the redox site was independent of the chromophoric center in this molecule.

Plans for next year include the initiation of a surface enhanced Raman spectroelectrochemical research project. Using the optical multichannel analyzer system with a Reticon (photodiode array) detector, the electrode reaction mechanisms of substituted pyridines on silver and mercury electrodes will be investigated by surface enhanced Raman spectroscopy (SERS). 
SERS is expected to yield new insights into electrode mechanisms by providing molecular spectra of adsorbed reactants, intermediates and products.

The major development in instrumentation will be the construction of a microprocessor which will be interfaced to several instruments to assist in data acquisition, experimental control, data reduction, and display. In addition, potentiostats are being constructed to provide individual group members with dedicated electrochemical control of their experiments.

Beyond this, our plans call for continued research on the projects noted above, e.g., (1) application of the CCES to chemical systems of interest such as the croconate compounds, (2) study of the spectroelectrochemical behavior of ceruloplasmin, (3) further improvements in electrochemical LC detectors, and (4) elucidation of the electrochemical behavior of various types of chemically modified electrodes. By these studies, we expect to develop the competences in organic electrochemistry which can be effectively utilized in the future to address analytical needs of the Center.

Richard A. Durst, Group Leader; Elmo A. Blubaugh, Kathryn A. Bunding, Lawrence M. Doane, Mary L. Fultz, William A. MacCrehan, William T. Yap

\section{Liquid Chromatography Group: Overview}

Research in the group continues to be focused on the analys is of polynuclear aromatic hydrocarbons ( $P A H^{\prime} \mathrm{s}$ ), phenols and nitrogen heterocyclic compounds which are important in the energy and environmental area, and anticonvulsant drugs and peptides which are important in the clinical and biomedical areas.

During the past year, we have utilized several procedures that were developed in previous years in the certification of two new Standard Reference Materials, SRM 1580 (Organics in Shale 0i1) and SRM 1644 (Generator Columns for Polynuclear Aromatic Hydrocarbons in Water). A high-performance liquid chromatographic (HPLC) fractionation procedure was used for isolating five phenols from a shale oil matrix prior to final quantitation by gas chromatography/mass spectrometry (GC/MS). A sequential HPLC procedure was used for the determination of six PAH's and two nitrogen heterocyclic compounds in SRM 1580. In addition, we have used sequential HPLC procedures in the measurement of several PAH's, nitrogen heterocyclics, and amino-PAH's in a petroleum crude oil and a solvent refined coal as well as tissue, air particulate, and sediment extracts. We also devoted some effort to the determination of polychlorinated biphenyls in waste and recycled oils.

The dynamic coupled column HPLC procedure developed in our group and used in the certification of the anthracene, benz(a)anthracene, and benzo(a)pyrene generator columns, is now also being utilized to determine ambient temperature vapor pressures and heats of sublimation of several PAH's. 
In the biomedical area, the strategies developed in previous years are being used in the analysis of a candidate Angiotensin I SRM. In addition, we have used these strategies for comparative analyses of Luteinizing Releasing Hormone (LRH), somatostatin, endorphins, and the hypothalmic peptide substance $P$ from several commercial sources. Alkaline rearrangement products of NAD have been isolated by preparative HPLC for NMR analysis. From these experiments, we have obtained sufficient data to propose an alternate reaction mechanism to the currently accepted one for the rearrangement.

Three independent HPLC procedures have been developed and are being evaluated for certification of valproic acid in human serum. This assay is particularly difficult because valproic acid is very volatile, does not contain an ultraviolet (UV) chromophore, and is not readily resolved from protein and other serum components on reverse-phase columns. The three procedures are: (1) a multi-dimensional procedure where the valproic acid is isolated from the serum on a precolumn and selectively eluted onto an analytical column for separation and quantitation with detection at $195 \mathrm{~nm}$; (2) a method based on the extraction and derivatization of the valproic acid so that it can be monitored at $254 \mathrm{~nm}$; and (3) analysis of underivatized serum extracts by LC-MS.

Some other noteworthy research efforts during the past year include: the development and evaluation of a quenchofluorometric detection system for chlorinated pesticides, and PAH's containing the fluoranthene moiety; investigation of the retention behavior of PAH's on both polymeric and monomeric HPLC bonded phases. In this context, it was observed that the retention of both parent and alkylated PAH can be predicted from the length to breadth ratio of the molecule; assessment of the stability of several phenols in distilled and industrial effluent waters under various conditions of storage; and direct quantitative LC-MS analysis of several phenols in shale oil and solvent refined coal.

In 1981, we plan to continue our present efforts in the environmental and biomedical areas and begin to develop expertise in food and nutritional analysis. In this context, we are sponsoring with FDA and USDA a Workshop on Reference Materials for Organic Nutrient Measurement. Specific research plans for next year include interfacing an optical multichannel analyzer (OMA) to a spectrofluorimeter in order to obtain fluorescence spectra of HPLC column effluents on-the-fly. Necessary optical modifications to the spectrofluorimeter have been made this year in preparation for this work. This project will be carried out in conjunction with the Organic Spectroscopy Group. In addition, we plan to develop a competence in chemical derivatization which will allow us to better measure compounds that do not have strong UV chromophores.

Willie E. May, Group Leader; Jeanice M. Brown-Thomas, Richard G. Christensen, Walter F. Kl ine, Sam A. Margolis, William J. Sonnefeld, Patricia A. White, Stephen A. Wise 
The major research emphasis for the Mass Spectrometry Group is in the development of mass spectrometric methods for the accurate quantitation of individual compounds in complex mixtures, development of high-resolution mass spectrometric techniques for the analysis of high molecular weight compounds, and continued development of a liquid chromatography-mass spectrometry (LC-MS) interface for the analysis of non-volatile analytes. Work during the past year has been concentrated in the environmental and clinical areas. During the past year, quantitative gas chromatography-mass spectrometry (GC-MS) methods have been developed and applied to the determination of individual analytes in complex samples such as shale oil, solvent refined coal, energy process effluents, and human serum. Several phenols and polynuclear aromatic hydrocarbons have been quantitated in a shale oil sample and the shale oil has been released as SRM 1580 .

Isotope dilution definitive methods have been developed for the quantitation of a number of human serum constituents including cholesterol, glucose, and uric acid. The methods result in the determination of the analytes with a precision of better than \pm 0.5 percent relative standard deviation (RSD). A human serum Standard Reference Material, SRM 909, is expected to be released in 1980 .

The high-resolution mass spectrometry facility is now fully operational. Accurate mass measurements above $\mathrm{m} / \mathrm{z} 1000$ are very important for the analyses of non-volatile compounds. Silver sulfonates have been used successfully for high mass calibration of high-resolution field desorption mass spectra to $\mathrm{m} / \mathrm{z} 1400$. High-resolution mass spectra of several polypeptides have been obtained using field desorption with photoplate detection and silver salts for mass calibration.

Work has continued this year on development and refinement of an LC-MS interface. A patent has been obtained for an LC-MS interface employing continuous sample preconcentration. Considerable refinements have been made in the design of the probe; these changes allow the use of aqueous mobile phases and allow introduction of an atomizer gas which can be either inert or a chemical ionization reagent gas. The LC-MS system has been used in the quantitative analysis of individual compounds in shale oil and solvent refined coal. Valproic acid, an anticonvulsant drug, has been quantitated in human serum with the LC-MS system operating in the chemical ionization mode using selected ion monitoring. Spectra of non-volatile compounds, such as adenosine, have been obtained using aqueous chromatographic mobile phases.

The chemical and isotopic purities of about thirty deuterated compounds have been evaluated for use as internal standards in the proposed Environmental Protection Agency (EPA) "Master Analytical Scheme" for priority pollutant analysis. Solutions of these internal standard mixtures have been supplied to EPA. Two of the internal standards, a deuterated naphthalenesulfonic acid and deuterated n-dodecyl phosphate, were not available commercially and were synthesized at NBS. 
Research planned for the coming year includes: continuation of the ongoing environmental projects with extension to new matrices and the investigation of negative ion chemical ionization for quantitation using recently acquired instrumentation; new isotope dilution definitive methods for urea and creatinine and the investigation of improvements to the instrumentation used; continued refinement and the beginning of application of high-resolution field desorption mass spectrometry; and changes in the NBS developed liquid chromatography/mass spectrometry system to improve its performance with polar compounds.

The competence-building program on "Analysis of Ionization Mechanisms and Ion Structures" will be initiated in 19.81. Specifically, two research programs will be started in the area of probing the structure of organic ions. The first project will involve laser induced metastable ion generation as a selective probe for molecular structure. The second project will involve collisional activation of ions with reactive gases as a means of directing fragmentation.

Edward White V, Group Leader; Rafael Espinosa, Laurence R. Hilpert, Stanley Meiselman, Kristy L. Richie, Lorna T. Sniegoski, Michael J. Welch

\section{Gas Chromatography Group: Overview}

The general thrust of research in the Gas Chromatography Group is aimed at multi-dimensional chromatographic techniques for use in quantitation of individual compounds in complex organic mixtures. Also, the possibility of utilizing combined gas chromatography-Fourier transform infrared (GC-FTIR) spectrometry to identify toxic organics in difficult matrices is being pursued.

Over the last year, the group has certified three additional phenols and two nitrogen heterocycles in the Shale $0 i 1$ Standard Reference Material (SRM 1580). The heterocyclic compounds were certified using the dual capillary multi-dimensional GC system developed last year.

The development of an analytical method for polychlorinated biphenyls (PCB) in recycled oils is almost complete. This has been aided by addition of a Hall conductivity detector to the group's equipment. The method developed utilizes both high-performance 1 iquid and gas chromatography, where liquid chromatography is used to remove analytical interferences prior to gas chromatographic quantitation. The use of the Hall detector removes the variation in detector response factors inherent in electron capture detectors.

A proposed ethanol-in-wine SRM has been certified and the recertifications of the heptane and iso-octane SRM materials have been completed. Gas chromatographic methods of analysis for the anticonvulsant drugs, carbamazepine and valproic acid, have been developed. In our program on the analysis of alternate fuels, a solvent refined coal liquid and a Wilmington petroleum crude $0 i 1$ have been analyzed for phenols and dibenzothiophene. 
A new on-column capillary injector (modeled after that published by K. Grob [1]) has been designed, built, and tested. Several new refinements have been added to our multi-dimensional GC capability. These include a new, low dead-volume, pneumatic switching valve, a dual capillary capability, and a more efficient oven configuration. The GC-FTIR system is now operational and the capillary interfacing is proceeding at a rapid rate; we can currently obtain good quality infrared spectra on many samples. A capillary, on-column injection technique has been devised for the analysis of trace levels of volatile halocarbons in drinking water. Al so, standards to be used by the Environmental Protection Agency's "Master Analytical Scheme" for water analysis have been prepared and their stability has been monitored.

During the coming year, the group plans to increase its capability in the area of multi-dimensional chromatography, including back-flushing and cyclic techniques so that more complex quantitations can be performed. Also, the FTIR-capillary system will be expanded and automated to make it easier to collect, manipulate, and store data from lengthy GC-FTIR chromatograms. In the future, we plan to acquire a microwave detection system for one of our gas chromatographs and subsequently be able to perform both organic and inorganic multi-element analyses on gas chromatographable samples.

\section{Reference}

[1] Grob, K., Journal of High Resolution Chromatography and Chemical Communications, 245 (1979).

Stephen N. Chesler, Group Leader; Sharon L. Bowie, Delmo P. Enagonio, Franklin R. Guenther, Reenie M. Parris 
B. Selected Technical Accomplishments

(Organic Analytical Research Division)

1. Isotope Dilution/Mass Spectrometry Definitive Method Developed for Creatinine in Serum

Elevation in serum creatinine signifies impaired kidney function. Its measurement is a useful diagnostic test since the relative creatinine concentration is fairly constant in individuals. Patients having kidney transplants are routinely monitored for creatinine for evidence of transplant failure. The commonly used method for measuring creatinine levels is based on the color produced by its reaction with alkaline picric acid. Unfortunately, a number of keto-acids found in serum also form colored products under these conditions and that elevates the apparent creatinine level. Methods to obviate this problem, e.g., sample pretreatment by chromatography, have given results that are also controversial. An isotope dilution/mass spectrometry (ID/MS) method already in the literature has not clarified the issue because the results it gives are imprecise and some possible sources of bias in the method were not investigated. Our ID/MS method is designed to overcome these uncertainties.

We synthesized creatinine labeled with three deuterium atoms in the methyl group that is attached to one of the ring-nitrogen atoms. This labeling avoids a potential isotope effect that could arise by use of creatinine having nitrogen-15 labeling ( ${ }^{15} \mathrm{~N}-1 a b e l e d$ creatinine was used in the published method). A derivative of creatinine, making it volatile, is needed for the gas chromatography step in the ID/MS method. In our method the creatinine is condensed with acetylacetone, and the product is then esterified to produce the known compound $\mathrm{N}$-(4,6-dimethyl-2pyrimidinyl)sarcosine, for GC/MS analysis. (In the published method, creatinine is treated with propylene oxide and then with trifluoroacetic anhydride to give a product whose exact structure is not known.)

The wet-chemical steps in our ID/MS method entail mixing a quantity of the creatinine- $d_{3}$ with an aliquot of a sample, separating the fraction containing the labeled and unlabeled creatinine from the sample by use of HPLC, and converting the creatinine into the sarcosine derivative. The mass spectrometric measurement to obtain the ratio of labeled and unlabeled molecules is initiated by injecting a small volume of the derivative mixture into a gas chromatograph that is equipped with a capillary column to effect a further purification, and then by directing the creatinine derivative into the mass spectrometer. Multiple ion monitoring (one ion each for the labeled and unlabeled forms of the creatinine derivative) is used to measure the ratio of the forms over the entire GC peak.

Known mixtures containing different proportions of SRM creatinine and the creatinine- $d_{3}$ are converted into the creatinine derivative for use as calibration mixtures. Two of these mixtures, having ratios of labeled and unlabeled creatinine closely bracketing the ratio found for the sample, are measured in sequence with the sample and in a second run are remeasured in the reverse sequence. Interpolation of the data 
provides the desired ratio. The concentration of the unlabeled creatinine in the sample is calculated from the ratio and from the known amount of labeled creatinine initially added.

The method has thus far been tested with only two samples. Each was analyzed as three independent replicates. The precision of the results ( $0.36 \mathrm{CV}$ ) is deemed adequate for a definitive method. By use of chemical ionization instead of electron impact ionization for generating ions, thereby obtaining other principal ions from which to measure the ratio of the forms of creatinine, we could find no evidence of interfering ions. Their presence would have led to different ratios being found. Additional serum samples must now be analyzed to assemble the data needed to establish the precision obtainable for the method and the absence of systematic error.

A. Cohen, H.: S. Hertz, R. Schaffer, L. T. Sniegoski, P. VanDerLijn,

M. J. Welch, E. White $V$

\section{Human Serum Reference Material (SRM 909)}

This SRM for clinical chemistry is certified for its concentrations of clinically important organic and inorganic analytes. NBS has used definitive methods because such methods afford analyte values that are highly precise and free from significant bias; thus, they are accepted as most accurate. In addition, the certificate accompanying the SRM will provide state-of-the-art values for the activities of several of the enzymes that are also present in the serum. These values are based on results obtained by several expert groups from clinical laboratories who jointly selected and used an optimized enzyme activity method for measuring each enzyme.

The freeze-dried serum is in the form of small, bead-like particles, quantities of which had been weighed into the vials. The uniformity in vial-to-vial content of freeze-dried serum was measured, by performing differential refractometry with a very sensitive instrument, on 170 vials of the serum after reconstitution. The between-vial variability was found to be within acceptable limits for a calibration reference material, according to Approved Standard ASC-2 of the National Committee for Clinical Laboratory Standards; i.e., the variability did not exceed \pm 1 percent of the mean value in more than 5 percent of the vials. Because a variability of that magnitude would lead to unacceptably high levels of uncertainty for definitive method value, that consequence was obviated by weighing the quantity of freeze-dried serum in each vial during the process of reconstituting the serum. The certified values and their uncertainties, which apply to 99 percent of the vials of the SRM with 95 percent confidence, are applicable when the entire content of each vial is reconstituted and are given per gram of freeze-dried serum.

Thus, users of the SRM will have to measure the mass of freeze-dried serum in each vial of SRM if they are to benefit from the accuracy of the certified values. A simple procedure for weighing the contents of dry serum in each vial is described in the certificate. 
If vials of the SRM are reconstituted without weighing the freezedried serum, the certificate provides the mean concentration values and the resultant wider limits of uncertainty that apply to 95 percent of the vials with 95 percent confidence.

The SRM is being issued even though the values for all the analytes and enzyme activities that are eventually to be reported have not been determined. Values will be sent to the users of the SRM automatically as soon as the work is completed for each additional analyte and enzyme activity.

Definitive method values based on isotope dilution/mass spectrometry are certified for glucose and uric acid. Values for cholesterol and urea are in process. The creatinine value will be determined as soon as the ongoing work to develop an ID/MS method for creatinine is completed. None of the enzyme activity values are ready to be reported at the time of the present writing (August 1980).

A. Cohen, R. G. Christensen, H. S. Hertz, D. J. Reeder, R. Schaffer, L. T. Sniegoski, M. J. Helch, E. White V

\section{Fluoroimmunoassay Procedure Developed for Dinitrophenols in Water}

Detection of trace amounts of the toxic pollutant 2,4-dinitrophenol in water is an analytical problem that presents many challenges to the analytical chemist. Laboratory investigations have shown that gas chromatography and high-performance liquid chromatography, while suited for analytical determinations of most of the toxic pollutants, are not as efficient in the separation and identification of the nitrophenols. The development of an immunochemical procedure for detection and quantitation of dinitrophenols in water has been the focus of efforts during 1980 .

Development of suitable reagents for the assay entailed the preparation of a suitable material (immunogen) to be injected into rabbits for production of antibodies. Highly purified bovine albumin was combiried with dinitrophenol and characterized. The immunogen most successful in eliciting antibodies had 23 dinitrophenyl groups per mole of albumin. The antiserum was produced under contract by a commercial supplier who injected rabbits with the prepared immunogen and bled them periodically for determining antibody development. A procedure that used double diffusion of antiserum versus the immunogen in a gel matrix was the preferred method for following antibody development.

Specific immunoglobulins (IgG) were separated from serum by use of an ion-exchange gel preparation. Purity of the IgG was ascertained by use of cellulose acetate strip electrophoresis. The IgG was then covalently bound to solid-phase polymeric beads by coupling with a carbodiimide reagent.

The antibody, thus attached to the beads, was able to bind 2,4-dinitrophenol. However, in order to use this capability in a quantitative assay, a compound that could act as a tracer needed to be synthesized. 
In order to be suitable, the tracer requires dual functionality, i.e., dinitrophenol reactivity as well as physical properties that can be measured, such as radioactivity or fluorescence. A dinitrophenolated fluorescent tracer with dichlorotriazinylaminofluorescein as the fluorescent moiety is currently being synthesized. Alternatively, the use of other fluorescein derivatives, such as fluorescein isothiocyanate, are being considered as substrates for dinitrophenylation.

The first tracer that we have had success in using in a quantitative competitive assay is a double-labeled rabbit albumin molecule to which both dinitrophenol groups and fluorescein isothiocyanate chromophores are covalently bound. In the competitive assay, the tracer and free dinitrophenol compete for antibody sites on the specific antibody solid-phase beads. Maximum sensitivity of the assay is determined by the amount of antibody-bound beads added, the quantity of the competing tracer in the mixture, and the time and temperature of assay incubation.

The solid-phase beads are separated by centrifugation, washed, and then counted in an instrument designed for photon counting, of fluorescence at $510 \mathrm{~nm}$ with excitation at $490 \mathrm{~nm}$. Binding curves are then generated from the resulting counts. Typical specific counts range from 30,000 to 600,000 in a two-second counting period, with background and control counts ranging from 15,000 to 25,000. Plots of the binding versus the amount of dinitrophenol added are linear over two orders of magnitude. Work is now focused on extending the sensitivity range of the assay.

By combining the specificity of the assay with capabilities of concentrating dinitrophenols with an affinity "accumulator" column, detection of ultra-trace amounts of dinitrophenol pollutants in water should be achieved. This will be of importance in the monitoring of industrial effluents.

P. Cooper, D. J. Reeder, R. Schaffer

4. Development of Methods for Determination of the Aromatic Carbon Content of Recycled 0ils by High-Field ${ }^{13} \mathrm{C}$ NMR Spectroscopy

Installation of the high-field NMR facility of the National Measurement Laboratory facilitated work on characterization of recycled oils as a result of the higher sensitivity and availability of more versatile pulse programming.

In efforts to improve the ${ }^{13} \mathrm{C}$ NMR method for measurement of aromatic carbon content, the use of carbon disulfide as a phase reference has been investigated. Carbon disulfide shows a narrow, well-defined ${ }^{13} \mathrm{C}$ resonance (see figure 13) and its addition to solutions of the oils in chloroform-d - tetramethylsilane has allowed more accurate phasing of the low lying aromatic ${ }^{13} \mathrm{C}$ envelope which increases the accuracy of integration. 


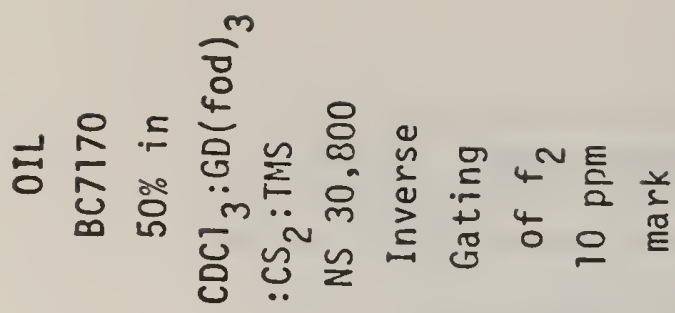

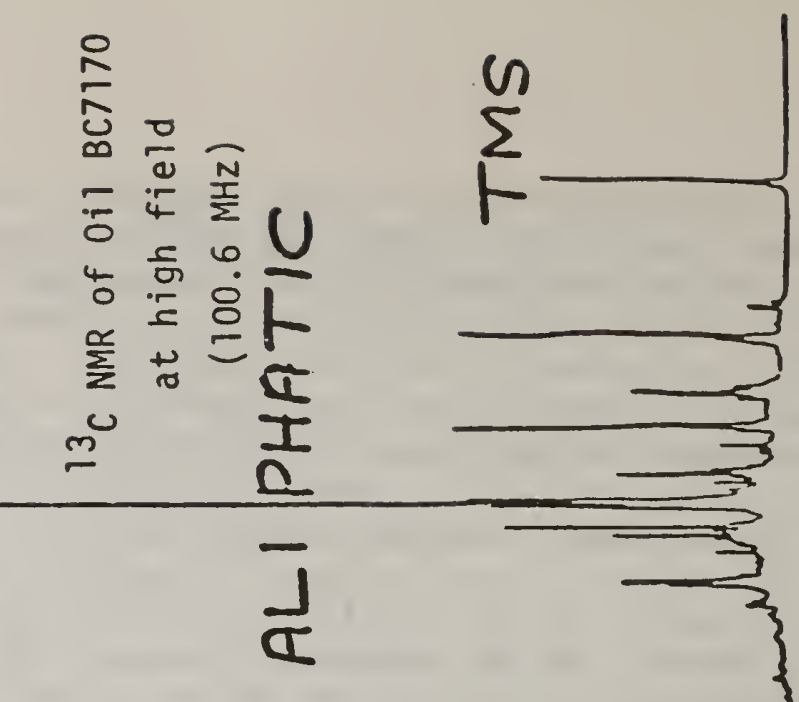

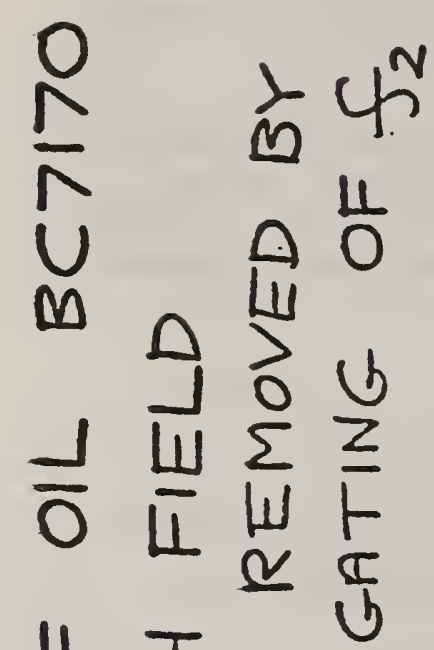

$\bigcup_{0}^{m}$

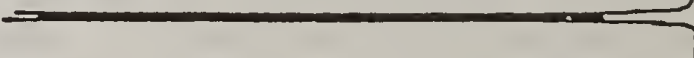

0

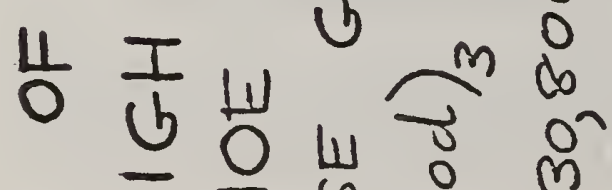
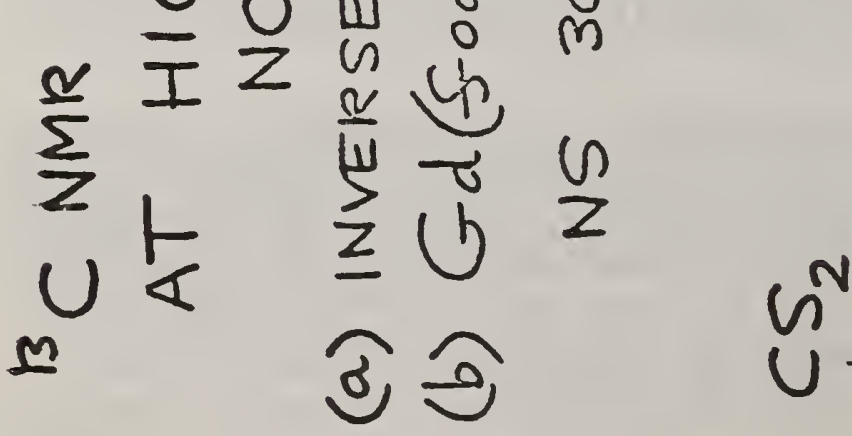

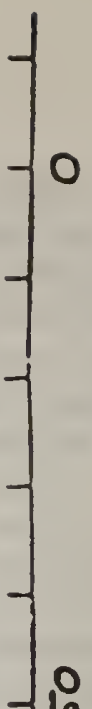

용

+

एक

రण

工

E "

안

언

웡늘 닐

은

u U⿺ 밍

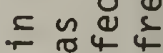

0 प

융

둥 =

u r

๑

등 꽁

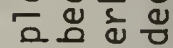

है

थ $>2 \frac{5}{4}$

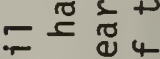

○ \& 4

b 문돈

$\frac{1}{5}=$

$\rightarrow \backsim$

U.

$<$ ○

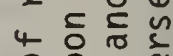

० 2

ᄃ

워

\pm 문

ב

웅 0 등

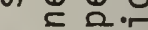

त

$4 \div 2$

0 品

ln 넌 प्ट है 枕

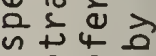
a d

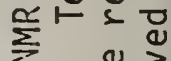

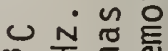

m 
Since variable nuclear Overhauser effects (NOE) can degrade the accuracy of quantitative measurements of quaternary aromatic carbon resonances, studies of two methods for removal of the NOE have been made: (a) inverse gating of the proton decoupling frequency and (b) the use of organic complexes of gadolinium as highly potent, paramagnetic relaxation reagents. Because removal of the NOE causes loss of sensitivity by up to a factor of three (resulting in a nine-fold increase in acquisition time), these studies have been conducted with the new, high-field superconducting NMR spectrometer, operating at $100.6 \mathrm{MHz}$ for ${ }^{13} \mathrm{C}$ and at $400 \mathrm{MHz}$ for protons. The ${ }^{13} \mathrm{C}$ spectra obtained show increased resolution in the aliphatic region and greatly increased resolution and definition of the weak signals in the aromatic region.

The solubility in chloroform of three organic complexes of gadolinium has been tested and the following order of solubilities found: gadolinium $(6,6,7,7,8,8,8 \text {-heptafluoro-2,2-dimethy } 7 \text {-3,5-octanedionate })_{3}$ $>$ gadolinium (acetylacetonate) $)_{3}>$ gadolinium (trifluoroacetylacetonate) $)_{3}$. On this basis, the octanedionate complex $\mathrm{Gd}(\mathrm{fod})_{3}$ was selected for the initial studies. For example, ${ }^{13} \mathrm{C}$ NMR analysis of oil sample BC7170 at high field using a combination of the inverse gating and $\mathrm{Gd}(\mathrm{fod})_{3}$ techniques yielded an aromatic carbon content of about 7 percent (see figure 13).

Because of the influence of ${ }^{13} \mathrm{C}$ spin-lattice relaxation times $\left(T_{1}\right)$ on quantitation, measurements of these parameters have been conducted at both low and high field (22.6 and $100.6 \mathrm{MHz}$, respectively). A stacked plot of recovery of the magnetization of the aliphatic ${ }^{13} \mathrm{C}$ nuclei at 22.6 $\mathrm{MHz}$ is shown in figure 14, and the $T_{1}$ values computed by a twoparameter exponential fit in Table 6 . The $T_{1}$ values of the ${ }^{13} \mathrm{C}$ nuclei represented by the 24 most intense resonances in the aliphatic carbon region range between 0.2 and 0.4 seconds, and the $T_{1}$ values measured for five bands in the aromatic carbon region range between 0.15 and 1.3 seconds and are given in Table 7 . The broad hump at $139.9 \mathrm{ppm}\left(T_{1} 1.3\right.$ sec) was assigned on the basis of its chemical shift to quaternary aromatic ${ }^{13} \mathrm{C}$ nuclei and the sharper bands at $129.0-125.7 \mathrm{ppm}$ to protonated aromatic ${ }^{13} \mathrm{C}$ nuclei. The shorter $\mathrm{T}_{1}$ values for the latter group are consistent with more effective dipolar relocation by the protons attached directly to these nuclei. 


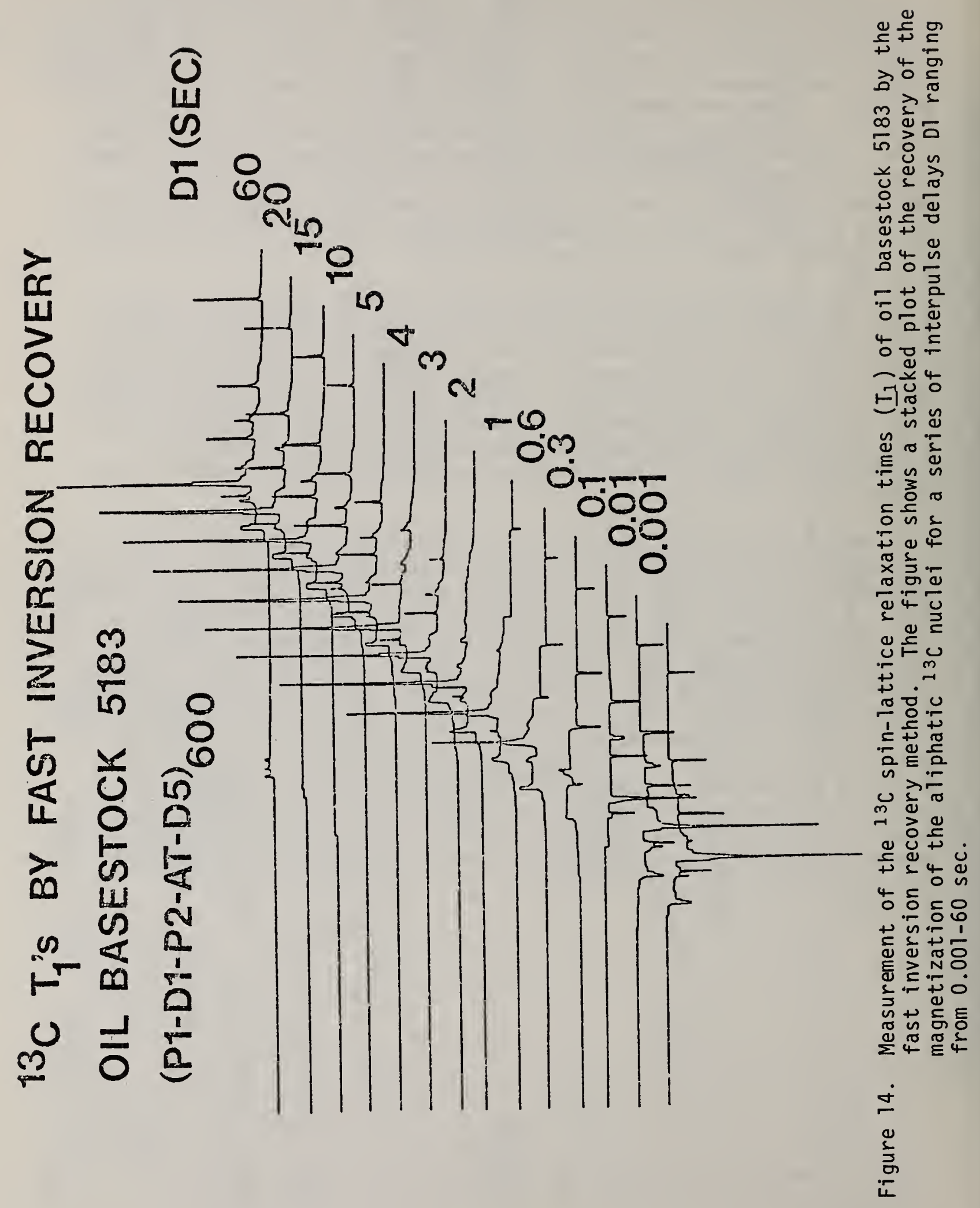


Table 6. Carbon-13 Spin-Lattice Relaxation Times $\left(T_{1}\right)$ of Aliphatic Carbons in 0ils Basestock \#5183.

Chemical

Shift

(ppm)

78.3

76.9

75.5

37.6

37.3

34.6

33.9

33.6

33.3

33.0

32.6

32.5

32.2

31.6

30.3

29.9

29.6

28.8

28.1

27.7

27.3

27.0

26.6

24.7

22.9

19.8

14.2

0
Percent

Intensity

(corrected)

3.4

3.2

1.7

1.6

1.9

2.2

3.8

1.9

2.0

5.6

1.6

6.0

23.3

8.4

2.2

2.6

1.8

3.1

2.5

1. 9

1. 9

7.4

3.9

6.0 \begin{tabular}{c}
$T_{1}$ \\
$(\mathrm{sec})$ \\
\hline
\end{tabular}

36.0

31.0

41.7

0.23

0.24

0.27

0.21

0.20

0.25

0.34

0.32

0.33

0.81

0.20

0.30

0.37

0.48

0.22

0.35

0.24

0.27

0.24

0.21

0.23

1. 31

0.68

2.39

11.06
Assignment

$\mathrm{CDCl}_{3}$

A.

L

I

P

H

A

$T$

I

c

C

A

R

B

0

N

S

$\mathrm{Me}_{4} \mathrm{Si}$ 
Table 7. Carbon-13 Spin-Lattice Relaxation Times $\left(T_{1}\right)$ of Aromatic Carbons in 0il Basestock \#5183.

$\begin{array}{lr}\begin{array}{l}\text { Chemical Shift Average } T_{1} \\ (\mathrm{ppm})\end{array} & \underline{(\mathrm{sec})} \\ 139.9 & 1.30 \\ 129.0 & 0.28 \\ 128.2 & 0.28 \\ 126.6 & 0.15 \\ 125.7 & 0.23\end{array}$

Assignment

Quaternary Aromatic ${ }^{13} \mathrm{C}$

Protonated Aromatic ${ }^{13} \mathrm{C}$

For measurements of ${ }^{13}{ }^{3} T_{1}$ values at high field $(100.6 \mathrm{MHz})$, the $T_{1}$ values were computed using a three-parameter exponential fit. Under these conditions, the ${ }^{13} \mathrm{C} \mathrm{T}_{1}$ values of the aliphatic carbons ranged between 0.5 and 2.0 seconds, and the values for the protonated aromatic carbons ranged between 0.2 and 0.6 seconds. These relaxation times are expected to assist interpretation of the quantitative results and the design of new experiments.

\section{B. Coxon}

5. Research on Two-Dimensional Proton NMR Spectroscopy and Its

Application to SRM D-Glucose

Implementation of two-dimensional, Fourier transform NMR spectroscopy on the new superconducting spectrometer (operating at $400 \mathrm{MHz}$ for protons) has indicated the tremendous potential of the method for the structural and quantitative analysis of organic materials that are of interest to NBS.

The method has been applied initially to a solution of D-glucose in methylsulfoxide- $d_{6}$, a type of solution that was used at NBS ten years ago for the analysis and characterization of this clinical SRM by continuous wave, proton NMR spectroscopy at $90 \mathrm{MHz}$. Three novel types of proton NMR spectra have been measured for D-glucose at $400 \mathrm{MHz}$ : (1) two-dimensional stacked plots (data matrix $8 \mathrm{~K} \times 128$ ) in which chemical shift and coupling constant information is separated and displayed in two different dimensions (see figure 15); (2) a proton decoupled, proton NMR spectrum obtained by rotation and projection of the data matrix onto the chemical shift axis (see figure 16); and (3) a series of cross sections ('J'spectra) of the two-dimensional NMR spectrum in which coupling constant information is displayed independently of chemical shifts. 


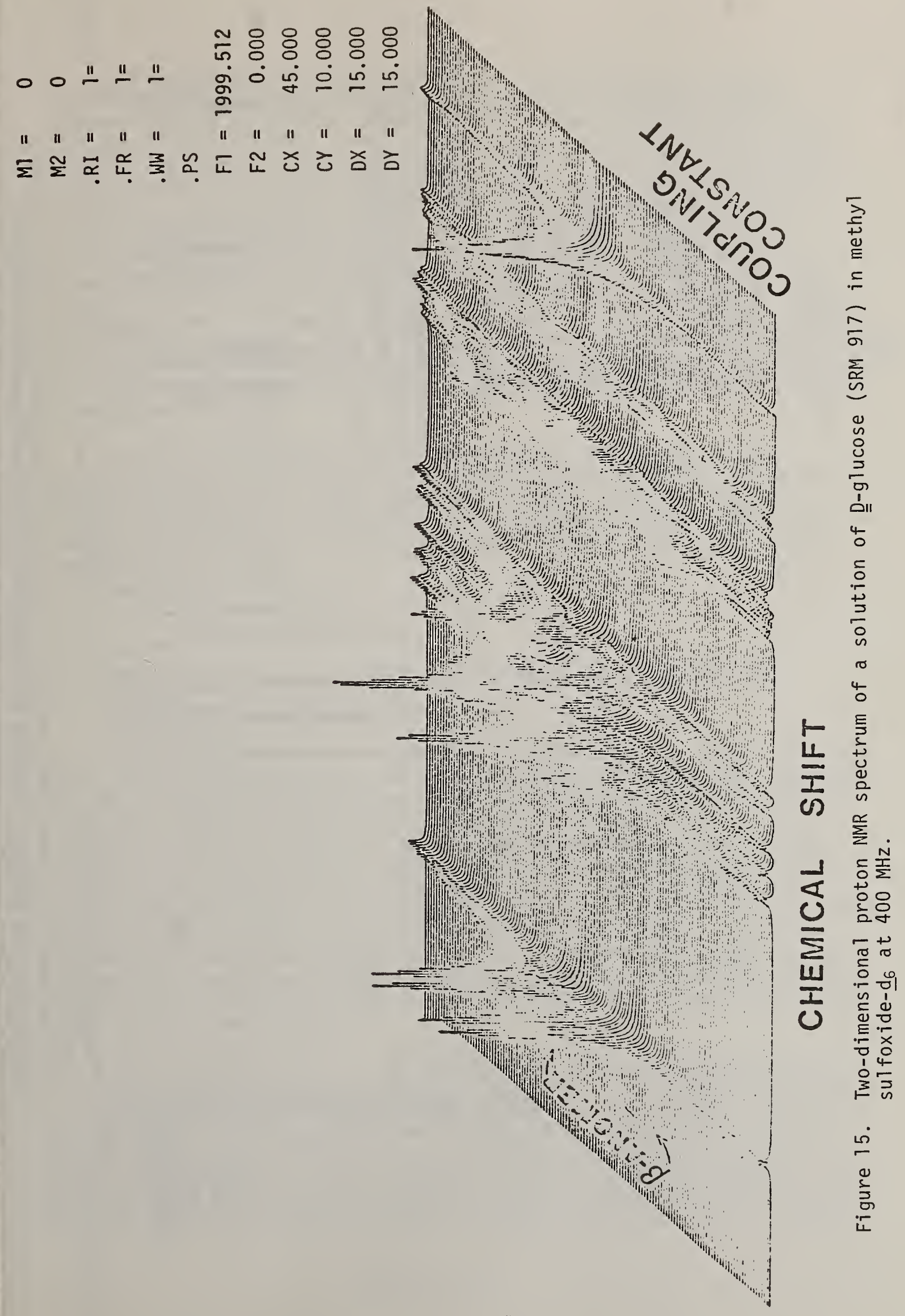




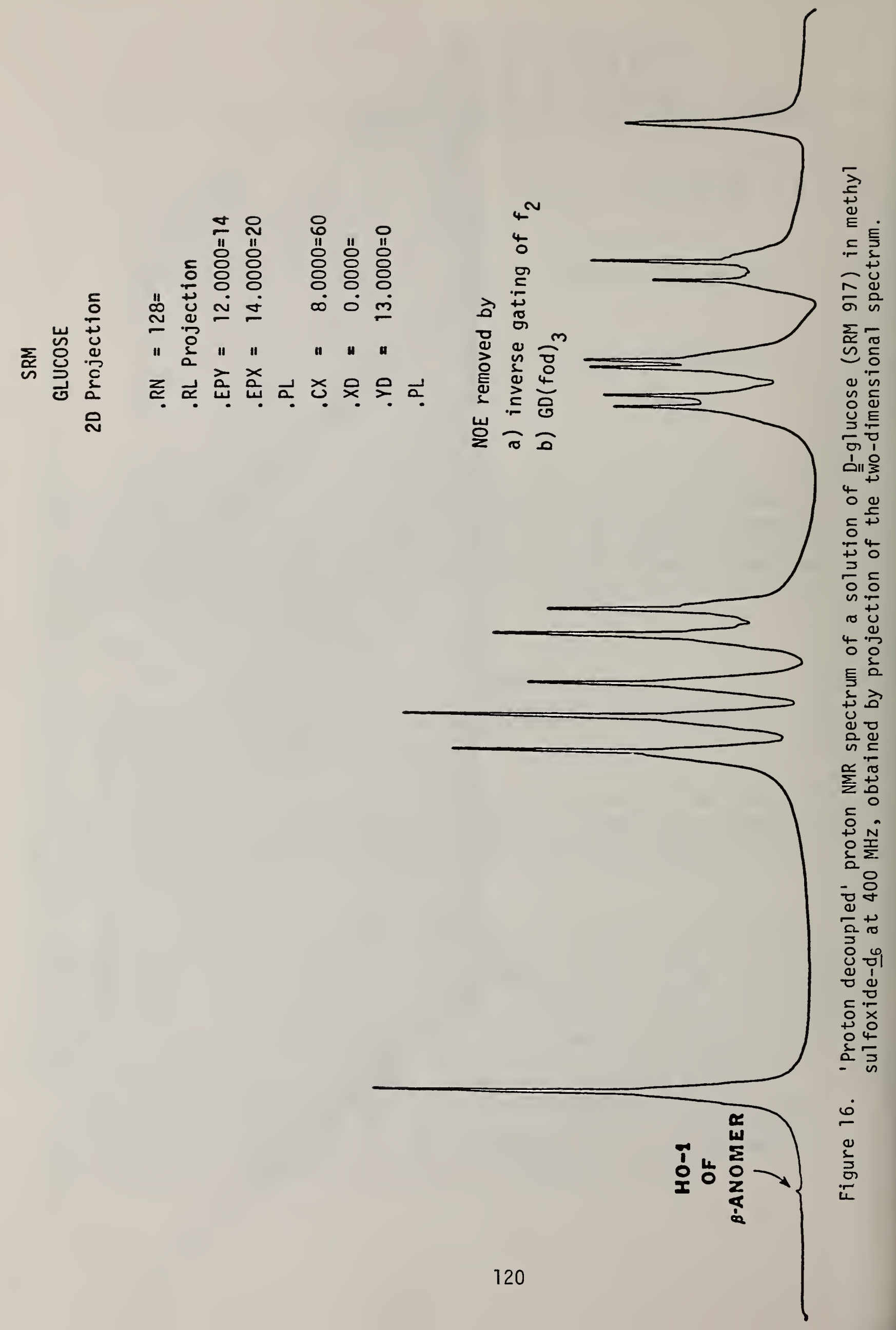


Proton NMR spectroscopy of D-glucose at $400 \mathrm{MHz}$ has revealed a wealth of spin coupling information that was not available from our earlier studies at $90 \mathrm{MHz}$. However, even in the one-dimensional proton NMR spectrum at $400 \mathrm{MHz}$, there is still some overlap of the proton signals of the complex twelve-spin system formed by the seven ring protons and five hydroxyl protons of D-glucose. The undesirable effects of this overlap are neatly removed in the two-dimensional method since the ' $J$ 'spectra are displayed in separated form. Since the method involves a spin-echo technique, refocusing of the magnetization vector removes the effects of magnetic field inhomogeneity, with the result that the ' $J$ '-spectra show resolution that is superior to that available in the one-dimensional spectrum, even after substantial resolution enhancement by Gaussian filtering. This has allowed more accurate measurements of several of the proton-proton coupling constants of D-glucose and also the unequivocal detection of several small couplings that have not been observed previously.

The proton decoupled, proton NMR spectrum (see figure 16) of D-glucose (obtained as the projection of the two-dimensional spectrum) displays twelve clearly separated singlets which indicate the great analytical potential of the method. The content of $\beta-\underline{D}-g l u c o s e$ in the SRM is indicated by the presence of a small singlet in the projection spectrum at low field.

\section{B. Coxon}

\section{Analysis of Sodium Pyruvate and Its Impurities by Proton NMR Spectroscopy at $400 \mathrm{MHz}$}

The high sensitivity and large dynamic range of the $400 \mathrm{MHz}$ spectrometer has facilitated development of a new method for quantitative analysis of sodium pyruvate which has been used to complete certification of the SRM. For example, this allowed 250 percent solutions of the sodium pyruvate in $9: 1 \mathrm{~V} / \mathrm{V} \mathrm{H}_{2} 0: \mathrm{D}_{2} \mathrm{O}$ to be analyzed effectively either with or without presaturation of the water resonance by narrow-band irradiation of the water protons prior to data acquisition. For the purpose of identifying impurities in the pyruvate, a solvent having a $10 \mathrm{w} \mathrm{D}_{2} \mathrm{O}$ content (necessary for field:frequency stabilization) is desirable, so as to minimize the deuterium exchange of labile $\mathrm{CH}$ protons (for example, the $\mathrm{CH}_{2}$ group in pyruvate oligomers). However, similar results were obtained by the use of $9: 1 \mathrm{H}_{2} \mathrm{O}:\left(\mathrm{CD}_{3}\right)_{2} \mathrm{SO}$, a solvent mixture which avoids the problems of deuterium exchange.

The high sensitivity of the spectrometer allows data acquisition for the analysis to be completed rapidly ( 7 minutes or less) before significant dimerization of the pyruvate has occurred. The results obtained for solutions at $\mathrm{pH} 6$ (the natural $\mathrm{pH}$ of the SRM) by integration of methyl signals (see figure 17) are shown in Table 8 . The methanol impurity in the pyruvate appears to be an integral part of the crystal structure since it is not readily removed by drying. The final column of Table 8 shows the increased proportion of dimer produced by subjection of the pyruvate 


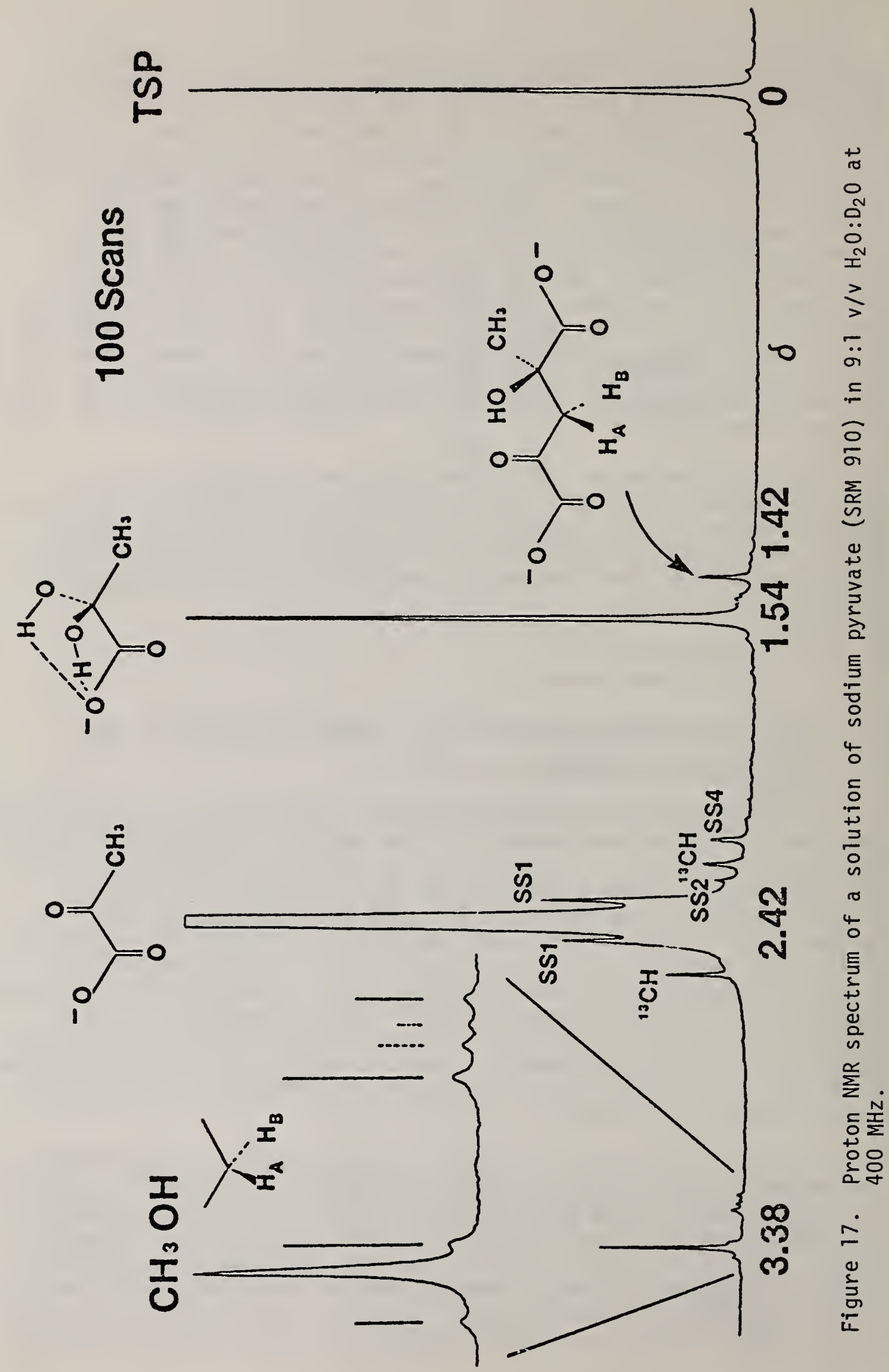




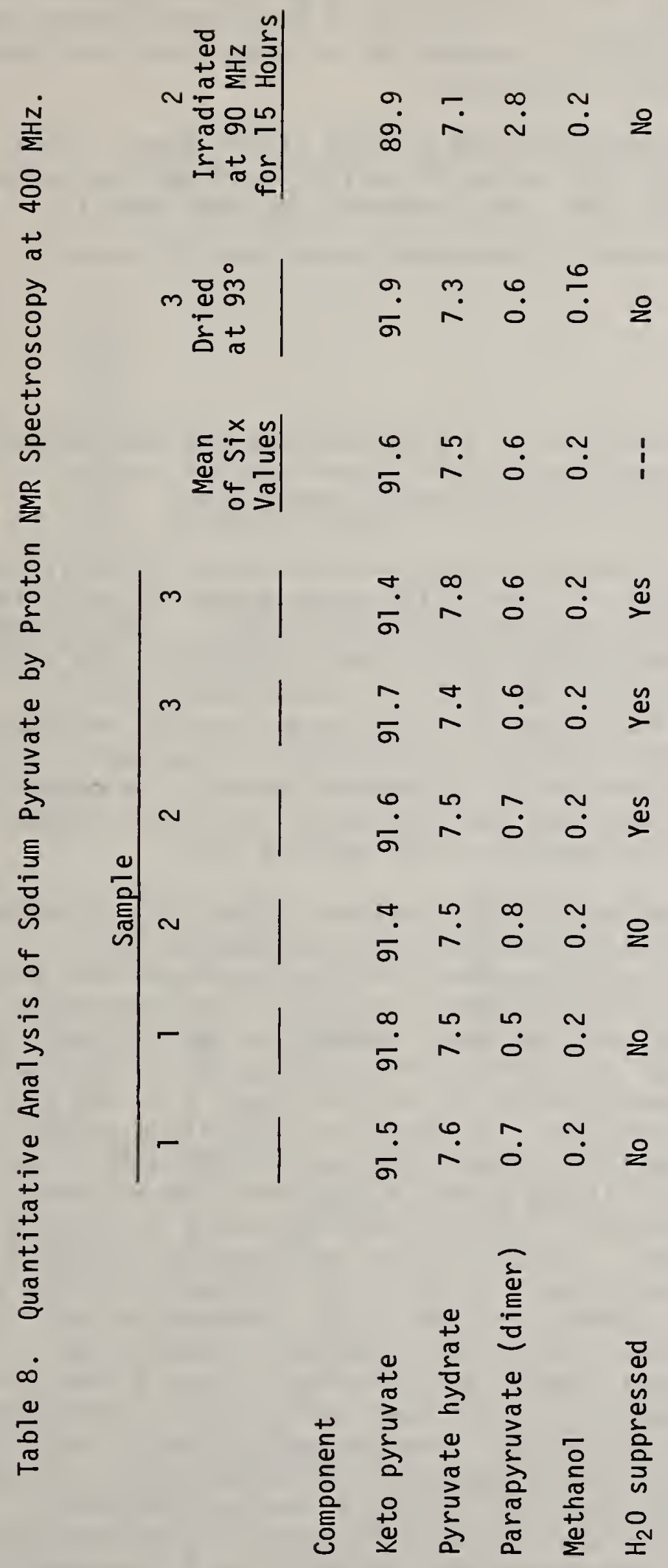


to the conditions of ${ }^{13} \mathrm{C}$ NMR spectroscopy at $22.6 \mathrm{MHz}$, during which, for the purpose of proton decoupling, the solution is broad-band irradiated at $90 \mathrm{MHz}$ for 15 hours. Analysis by proton NMR is much faster and avoids any problem due to RF heating of the sample.

The non-equivalence of the methylene protons of the dimer I (see figure 18) is striking at $400 \mathrm{MHz}$ and an $A B$ analysis yielded the parameters $\delta_{A B} 51.3 \mathrm{~Hz}$ and $J_{A B} 18.1 \mathrm{~Hz}$. This research has been done in conjunction with the development of improved separations of pyruvate dimer by HPLC.

B. Coxon, S. A. Margolis

7. Analysis of Boat Conformations of $\alpha-\underline{\underline{D}}-A 1 t r o p y r a n o s i d e ~ D e r i v a t i v e s$

by Interpretation of Highly Coupled Proton NMR Spectra

Bicyclic derivatives of the rare sugar altrose are of interest as intermediates in the synthesis of ${ }^{15} \mathrm{~N}$ labeled amino-sugars in which the ${ }^{15} \mathrm{~N}$ isotope has a well-defined stereochemical orientation. These isotopically labeled derivatives are being used in basic studies of the angular dependence of ${ }^{15} \mathrm{~N}$-proton coupling constants, a relationship which is expected to facilitate the NMR analysis of the aminoglycoside antibiotics. The availability of a $400 \mathrm{MHz}$ proton NMR spectrometer has allowed, for the first time, complete dispersion of the proton spectra of a number of $\alpha$-D-altropyranoside derivatives that had previously resisted complete anālysis at such lower field strengths as 100 and $220 \mathrm{MHz}$.

Complete analysis of the proton NMR spectrum (figure 19) of methyl 4,6-0-benzylidene-3-deoxy-3-phthalimido- $\alpha$-D-altropyranoside (I) at $400 \bar{M} \mathrm{~Hz}$ has revealed vicinal proton-proton coupling constants that are substantially different from those measured previously for many other $\alpha$-D-altropyranoside derivatives. The first order coupling constants for I (see Table 9) indicate that its pyranose ring adopts the ${ }^{5} B^{2}(\underline{D})$ boat conformation in which the substituents at $C-1, C-2, C-3, C-4$, and $C-5$ all have the equatorial orientation. By contrast, the values of the vicinal proton coupling constants for methyl 3-amino-4,6-0-benzylidene3-deoxy- $\alpha$-D-altropyranoside (II) and methy1 4,6-0-benzylidene-2-deoxy2-phthal imîno- $\alpha-\underline{D}-a 1$ tropyranoside (III) confirm that these derivatives adopt the ${ }^{4} C_{1}(\underline{D})$ conformation. Evidently, the conformational locking effect of the phthalimido substituent is not sufficient to cause III to adopt a boat conformation. However, in the ${ }^{4} \underline{C}_{1}(\underline{D})$ conformation of $I$, this effect is combined with a 1,3-diaxial interaction between the phthalimido and methoxyl substituents, with the result that I favors a boat conformation. Stabilization of this boat conformation is possible by hydrogen bonding between the hydroxyl and carbonyl groups. This is supported by the observation of less extreme coupling constants for the 2-0-acetyl and 2-0-benzoyl derivatives (IV and $V$, respectively) of I (see Table 9). An alternative class of boat conformations is represented by methyl 2,6-anhydro- $\alpha$-D-altropyranosides, in which the 2,6-anhydro ring forces adoption of a boat conformation $\left[{ }^{5} B^{2}(\underline{D})\right]$ in which all substituents 

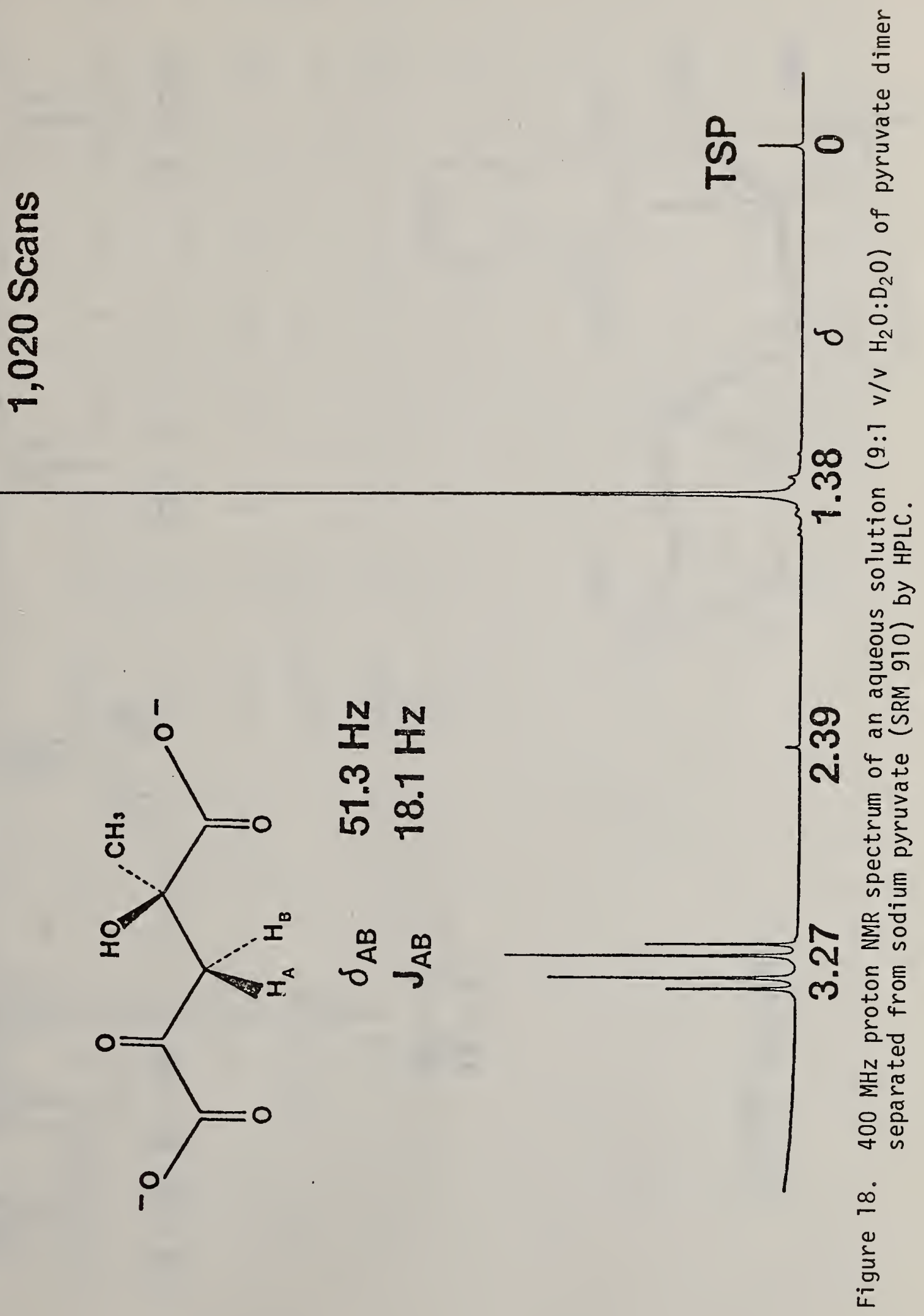


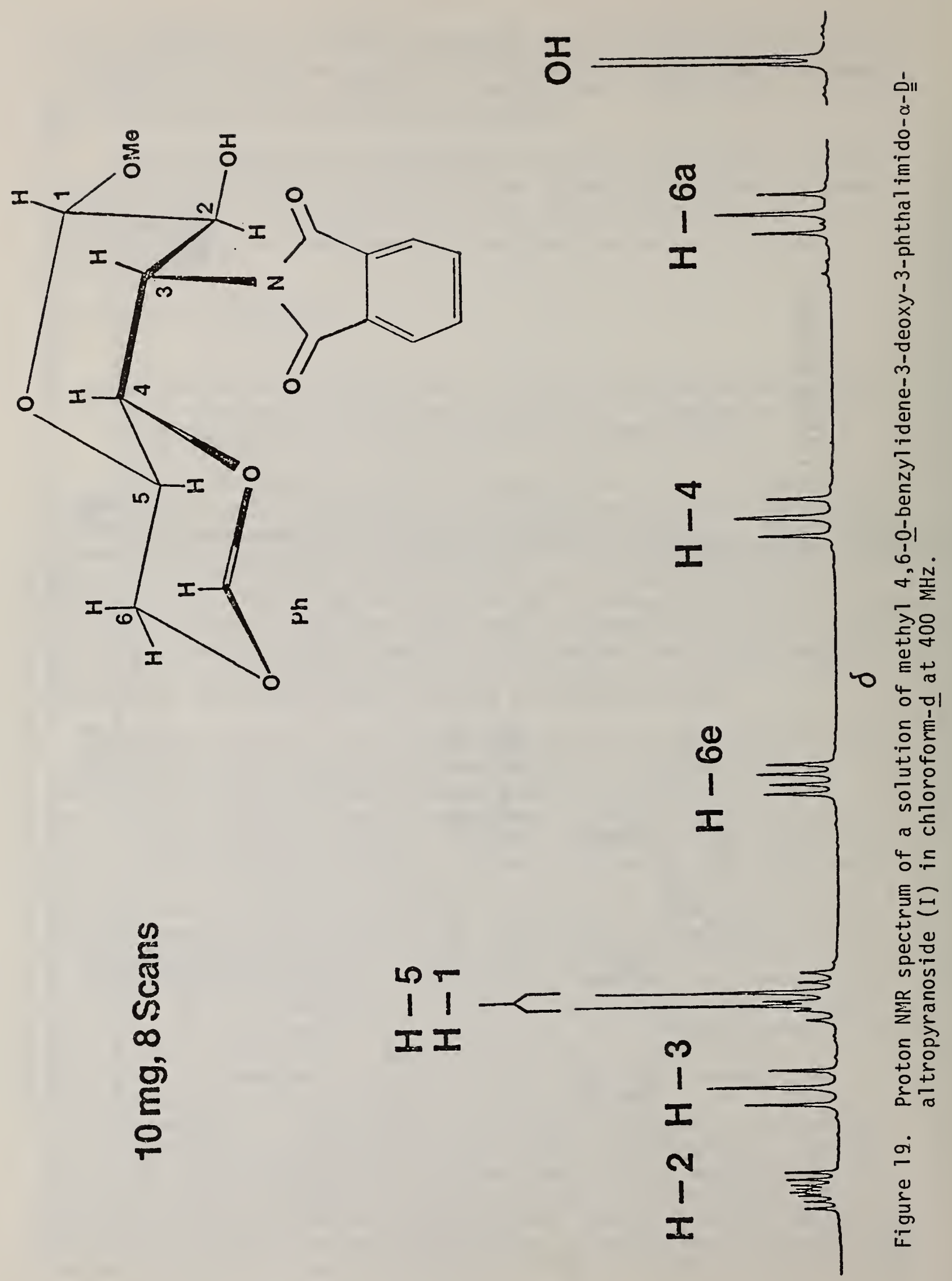




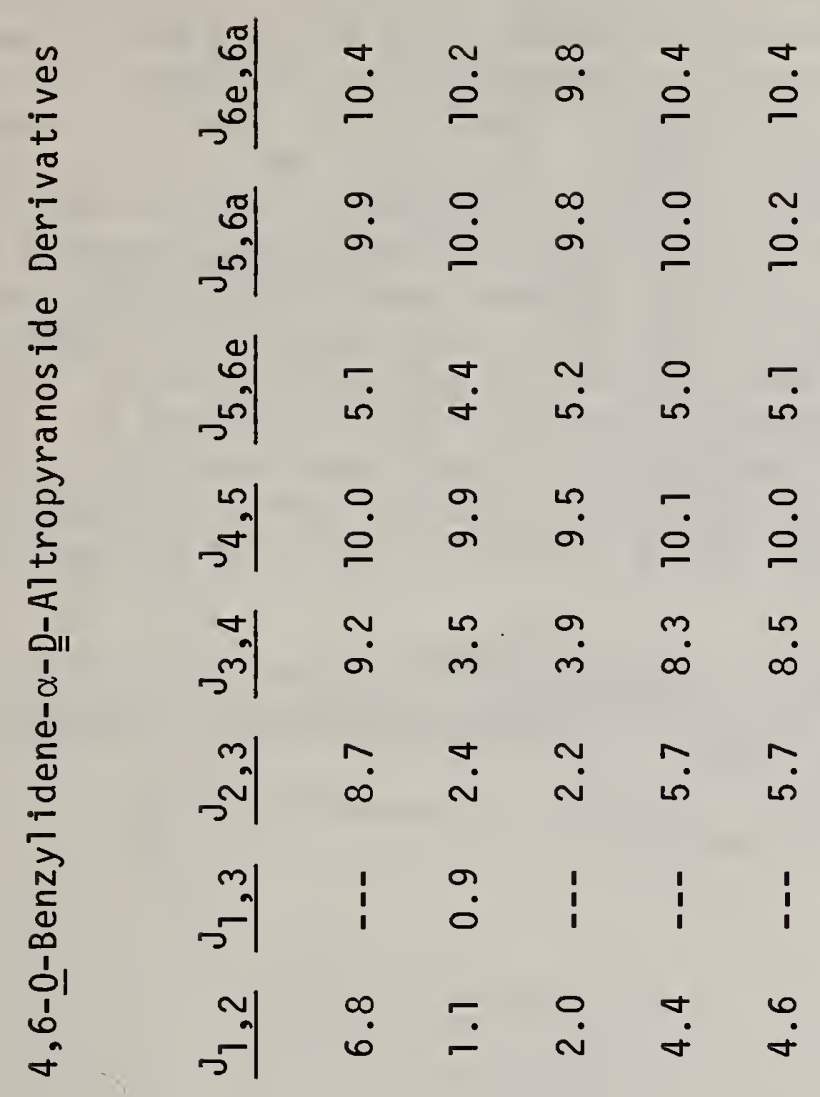

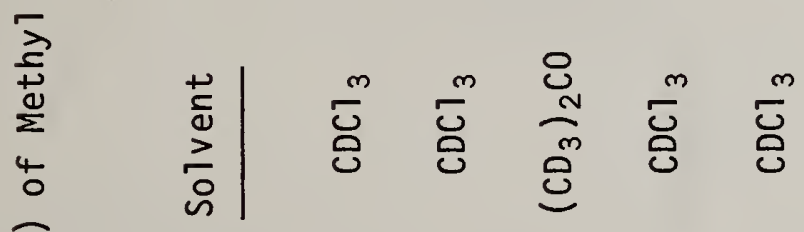

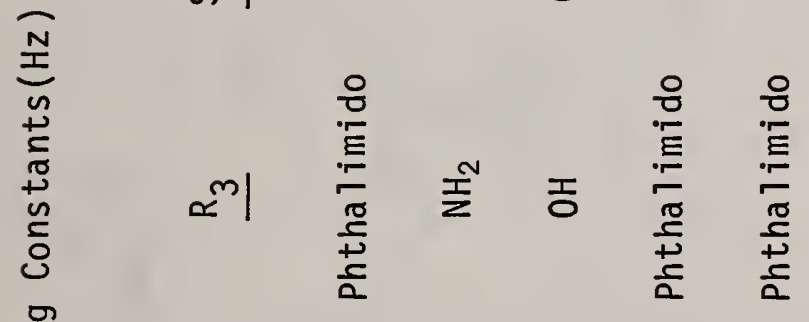

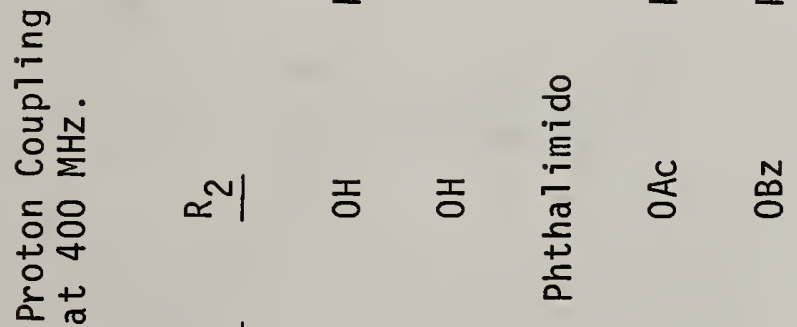

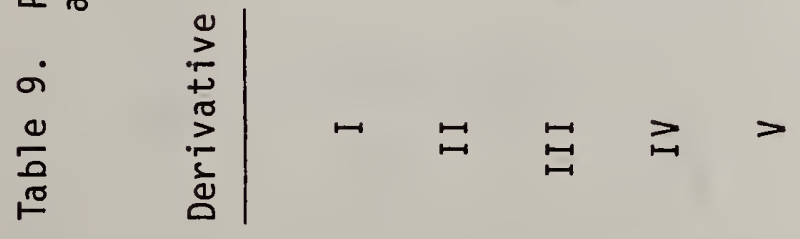


are axial. Proton NMR at $400 \mathrm{MHz}$ has now made conformational analysis of this unique bicyclooctane-like ring system feasible for the first time.

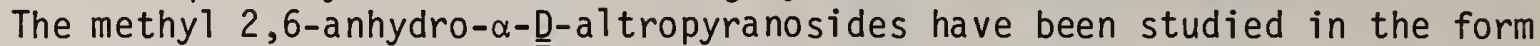
of the 3,4-diol (VIII), its di-0-acetyl derivative (VI), and the recently synthesized 3,4-anhydro derivative (VII). The proton NMR spectrum of the latter 2,6:3,4-dianhydro derivative (VII) at $400 \mathrm{MHz}$ is extraordinarily complex due to the presence of a large number of long-range coupling constants (see figure 20a and Table 10). The many small long-range coupling constants $(<1 \mathrm{~Hz})$ were detected by resolution enhancement techniques using Gaussian filtering of a $64 \mathrm{~K}$ data set and were confirmed by computerized simulation of the seven-spin system (figure 20b) and by homonuclear decoupling. The large number of long-range coupling constants of VII may be attributed to enhanced transmission of spin coupling information through the electron delocalization or multiple pathways of the epoxide (3,4-anhydro) ring.

We plan to use the dianhydride (VII) for the synthesis of $15 \mathrm{~N}$ labeled amino-sugars in which the $15 \mathrm{~N}$ and proton nuclei have the rare dihedral angles of either $0^{\circ}$ or $120^{\circ}$.

B. Coxon, M. L. Luzarraga, R. C. Reynolds 


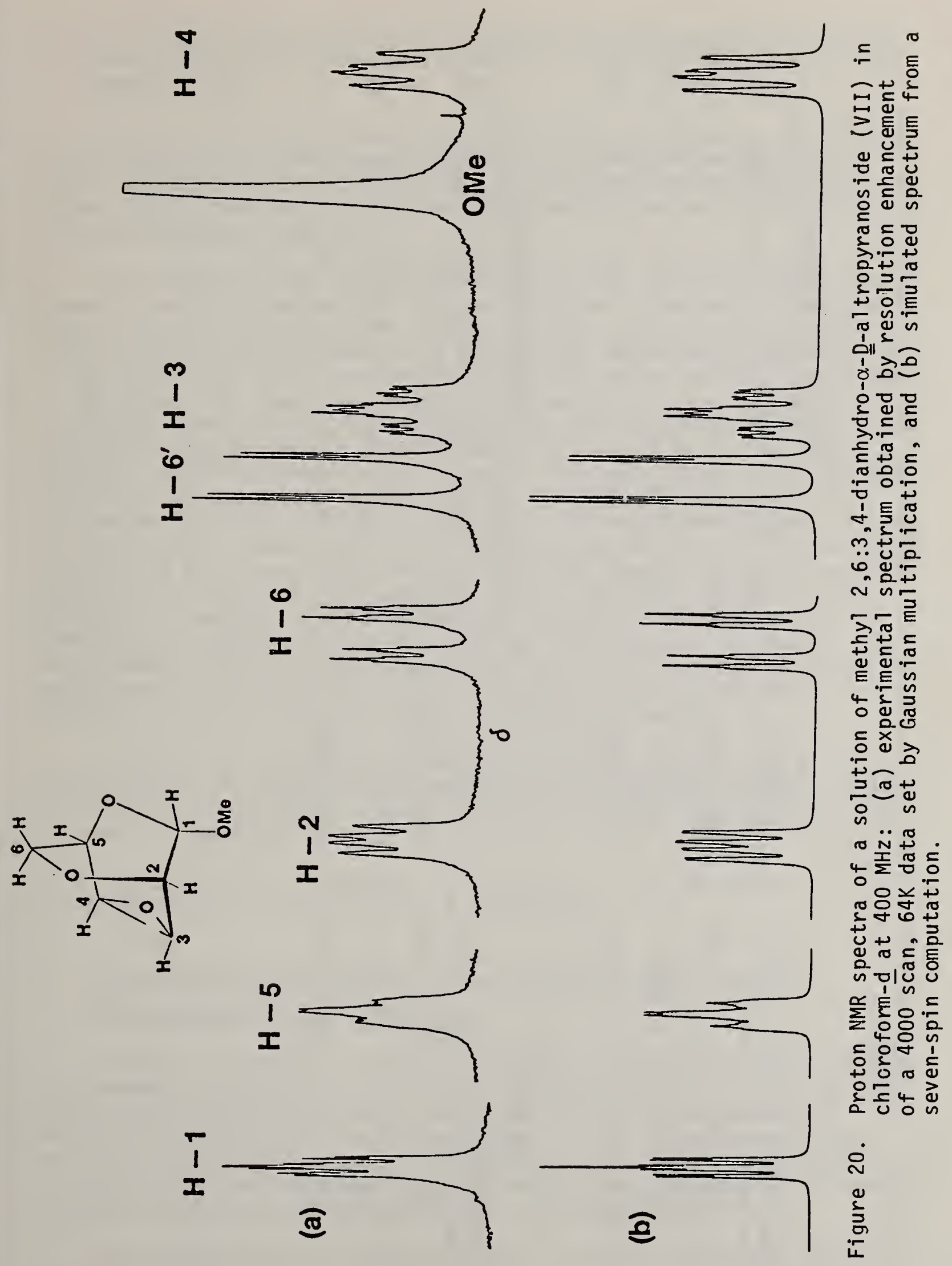




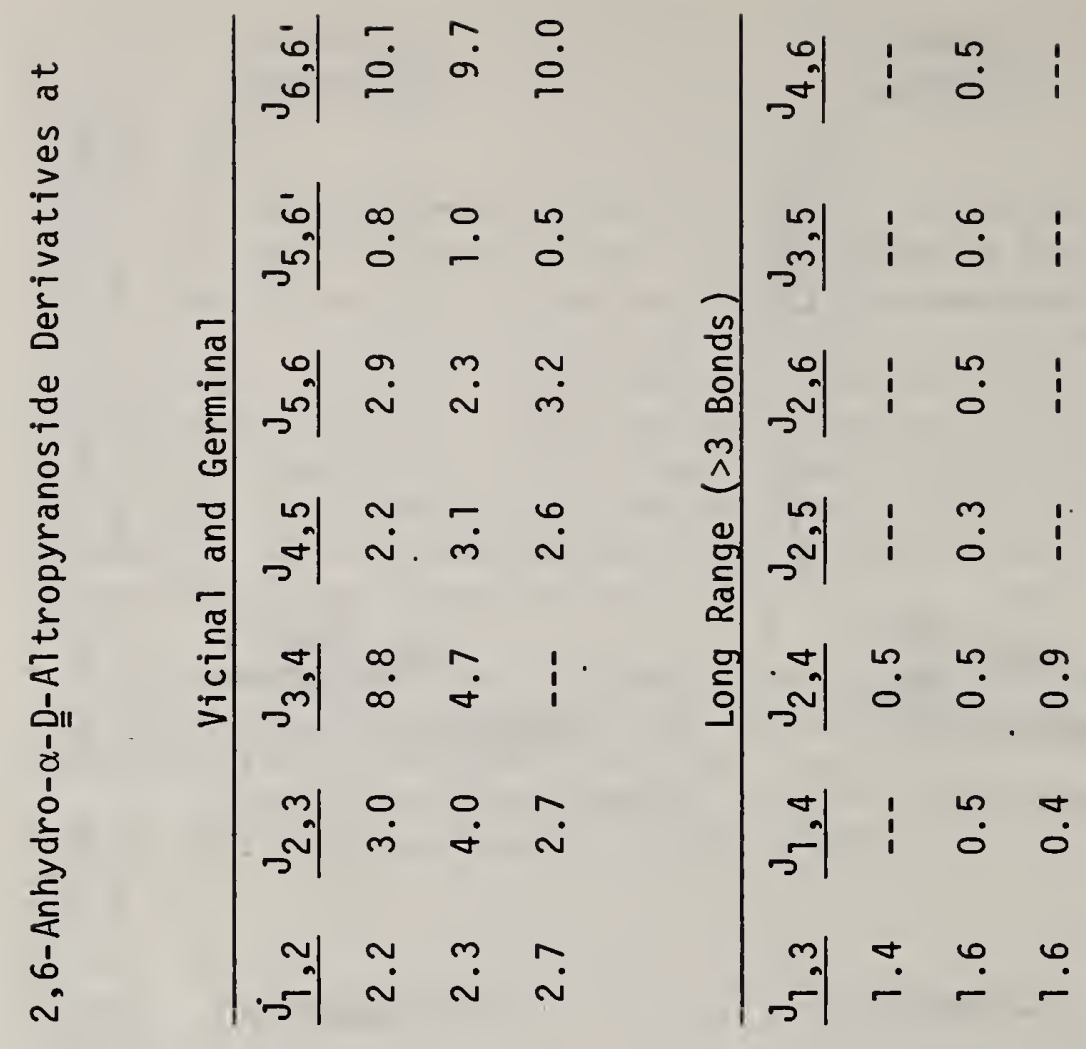

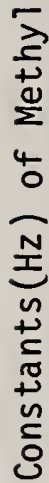

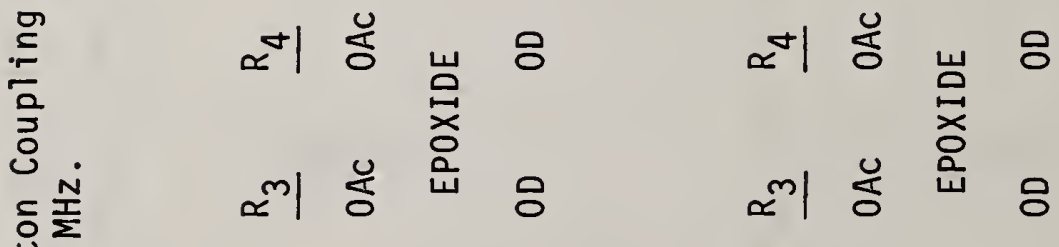

웅용

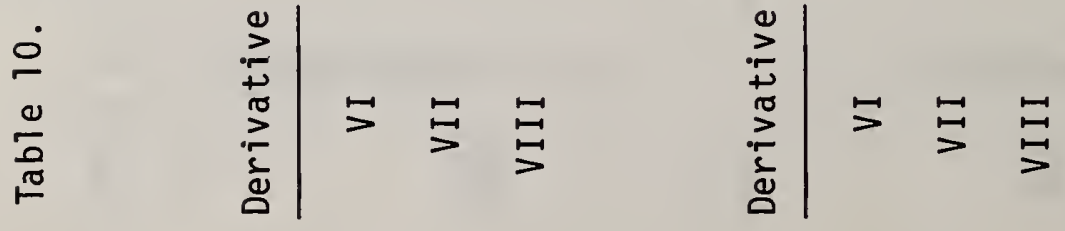




\section{Laser Polarimetry and Its Application to Recertification of Sucrose SRM (SRM 17c)}

Due to instrumental advances and more accurate moisture determination techniques of recent years, industrial and academic groups have found needs for more precise and accurate certified optical rotation values. This resulted in the recertification of the sucrose Standard Reference Material, SRM 17c.

To accomplish this, we determined the optical rotatory dispersion of standard quartz plates and SRM sucrose solutions using the high-accuracy polarimeter designed and built at NBS. Argon, helium-cadmium, heliumneon, and dye lasers, as well as a mercury arc were used as light sources in these studies. Our experimental results at different wavelengths were in agreement with results calculated from empirical formulas for the wavelength dependence of the optical rotations. Extensive determinations of optical rotation values were made for both quartz plates and SRM sucrose. Solutions using both the mercury arc with filter pack ( $546 \mathrm{~nm})$ and the helium-neon laser $(632.8 \mathrm{~nm})$ showed excellent agreement and further verified the validity of the empirically derived rotatory dispersion equations. Thus, the optical rotations of the SRM sucrose and quartz plates at a given wavelength may be calculated from the optical rotation determined at $632.8 \mathrm{~nm}$. In addition, use of the laser in place of the mercury arc reduces the measurement time by an order of magnitude. We, therefore, recommend the use of lasers as light sources in general and, more specifically, the helium-neon laser as a light source in routine polarimetry because its stability, increased intensity, and monochromaticity result in more precise and accurate measurements.

In addition to certifying the optical rotation, we are completing certification of the physical parameters of density and refractive index for the SRM. These data, along with certified values for purity will be of great interest to industry.

R. V. Bostelman, A. L. Cummings, D. K. Hancock, S. A. Margolis,

D. J. Reeder, L. T. Sniegoski, R. A. Velapoldi

9. Certification of Generator Columns for Polynuclear Aromatic

Hydrocarbons in Water (SRM 1644)

In the fall of 1975, the National Bureau of Standards (NBS) and the Environmental Protection Agency (EPA) jointly sponsored a series of workshops entitled "Standards and Reference Materials for Environmental Analys is Associated with Energy Development". The objective of these workshops was to obtain input from experts in the field of the methodology and certified standards needed for the accurate analysis of environmental samples associated with the production of alternate fuels.

At the conclusion of these workshops, a number of Standard Reference Materials (SRM's) were recommended by the participants for NBS consideration. One of the SRM's recommended was a polynuclear aromatic hydrocarbon 
(PAH) in a water matrix. Although many PAH's have demonstrated mutagenic properties, this SRM was given a low priority because of the presumed difficulties associated with the preparation and stabilization of such a material.

Shortly thereafter, we discovered that saturated solutions of PAH could be produced by simply passing distilled water through a column packed with glass beads coated with the compound of interest. The aqueous solubility of a compound is a well-defined thermodynamic quantity. Therefore, saturated aqueous solutions produced by these "generator columns" may also be defined as standard solutions at a given temperature.

Generator columns for anthracene, benz(a)anthracene, and benzo(a)pyrene constitute SRM 1644. The columns were prepared commercially and are currently being certified by two independent analytical techniques.

The first technique involves quantitative extraction of the PAH from the aqueous stream by an "extractor column" packed with $C_{18}$ bonded phase, use of an acetonitrile-water eluent to transfer components from the extractor column to an analytical $C_{18}$ column for separation of the analyte from non-analyte components, and detection of the analyte by absorbance at $254 \mathrm{~nm}$.

The second technique uses a "standard addition" technique for "on stream" analysis. The effluent from the generator column is mixed with $\mathrm{PAH}$ standards dissolved in acetonitrile, and the resultant $\mathrm{PAH}$ in the mixed solvent determined by fluorescence.

Preliminary data from the certification are given in Table 11. Upon completion of the certification process, one equation will be derived from a compilation of the data obtained from the two methods which will allow the user to interpolate the certified concentration between $10{ }^{\circ} \mathrm{C}$ and $30{ }^{\circ} \mathrm{C}$. This SRM will be certified and available by October 1980. 
Table 11. Preliminary Results of Anaalys is Using Two Techniques for Generator Column Aqueous Effluents.

Anthracene

Temperature

$\left({ }^{\circ} \mathrm{C}\right)$

30

25

20

15

5

Temperature

$\underline{\left({ }^{\circ} \mathrm{C}\right)}$

30

25

20

15

Temperature

$\left({ }^{\circ} \mathrm{C}\right)$

30

25

20

15

10

\begin{tabular}{lc}
\multicolumn{2}{c}{ Concentration (ppb) } \\
\hline Technique 1 & Technique 2 \\
58.8 & 59.6 \\
42.8 & 42.9 \\
31.1 & 30.9 \\
22.6 & 22.2 \\
& \\
Benz (a) lanthracene &
\end{tabular}

\begin{tabular}{ll}
\multicolumn{2}{c}{ Concentration (ppb) } \\
\hline Technique 1 & Technique 2
\end{tabular}

12.3

13.0

8.86

9.32

6.37

6.67

4.58

4.78

Benzo (a)pyrene

\section{Technique 1}

2.28

1.62

1.14

0.80

0.57
Concentration (ppb)

Technique 2

2.26

1.60

1.14

0.81

0.58

J. M. Brown-Thomas, W. E. May, W. J. Sonnefeld, R. A. Velapoldi,

P. A. White 
10. First Natural Matrix Trace Organic SRM Issued for Shale 0il (SRM 1580)

The current energy demand and the inevitable depletion of our petroleum reserves has resulted in a national program with a goal to insure an adequate supply of energy having minimum impact on public health and the environment. The conversion of coal to gaseous or tiquid fuels and the utilization of oil shale and tar sands are some of the energy sources that appear promising because of their relative abundance. To evaluate the potential environmental consequence of these activities, it will be necessary to analyze a feedstock, process streams, plant effluents, and final products for trace element and organic compound content. The results of these analyses cannot be assessed adequately without knowledge of the accuracy of the analytical techniques used to obtain the data.

We have in the past conducted several collaborative studies aimed at assessing the accuracy of data obtained from trace organic analytical methodologies. The results of a recent study involving the determination of specified phenols, polynuclear aromatic hydrocarbons, and $\mathrm{N}$-heterocyclics in a shale oil sample by several laboratories revealed large systematic biases among laboratories. One method for enhancing the accuracy of analytical measurements is the use of suitable quality assurance standards or Standard Reference Materials (SRM's). However, until now, SRM's for environmental trace organic analyses were nonexistent due to the lack of analytical methodologies necessary for certification.

Over the past few years, analytical methodologies have been developed and used to certify the first natural matrix SRM for trace-level organic constituents, Organics in Shale 0i1, SRM 1580. The quantitation of individual compounds in the complex shale oil matrix necessitated the use of multi-dimensional chromatographic procedures, i.e., gas or liquid chromatography on two columns of different selectivity and/or extremely selective detection methods.

Three different methods of sample preparation were used prior to analysis: simple dilution of the shale oil with methylene chloride (or other suitable solvent); acid/base extraction to isolate acidic, basic, and neutral components; and a high-performance liquid chromatographic fractionation. Two of the following techniques were employed to determine the certified values for the organic compounds in shale oil: capillary gas chromatography (GC), gas chromatography/mass spectrometry (GC/MS) with single ion monitoring for selective detection, and highperformance liquid chromatography (HPLC) with selective fluorescence detection. Al1 GC/MS analyses used the standard addition method for quantitation. The GC and HPLC analyses employed either internal standard, external standard, or standard addition methods. The excellent comparability of the values determined by these different analytical methods is illustrated in Table 12. Table 13 lists values for other compounds determined by only one technique. These values will appear on the SRM certificate for information only. The methods developed for the certification of this SRM are applicable to the determination of similar compounds in other complex natural matrices. Standard Reference Material 


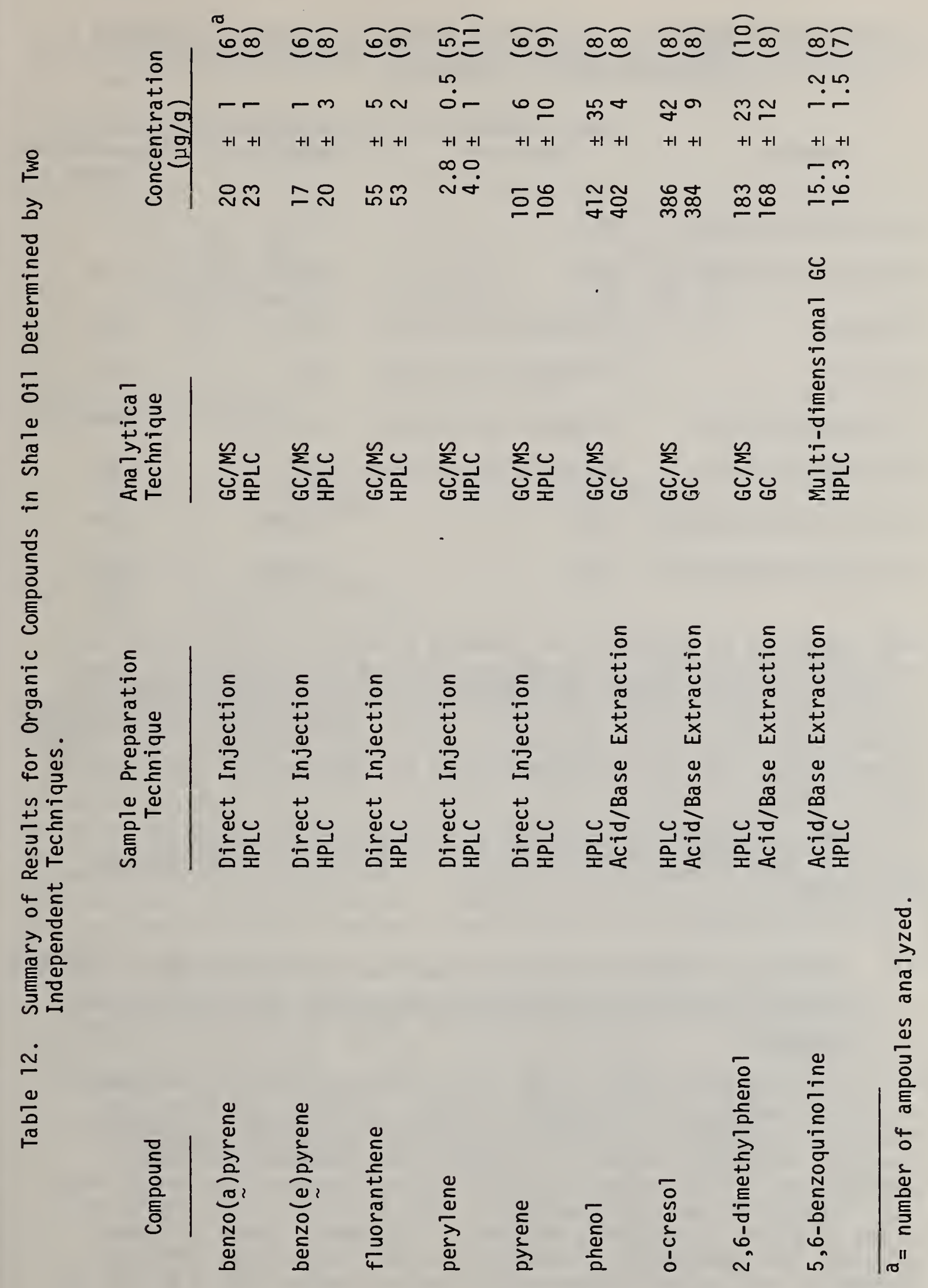


Table 13. Summary of Results for Organic Compounds in Shale 0il Determined by One Technique.

\begin{tabular}{|c|c|c|c|}
\hline Compound & $\begin{array}{c}\text { Sample Preparation } \\
\text { Technique }\end{array}$ & $\begin{array}{l}\text { Analytical } \\
\text { Technique }\end{array}$ & $\begin{array}{c}\text { Concentration } \\
(\mu \mathrm{g} / \mathrm{g})\end{array}$ \\
\hline benzo(k)fluoranthene & HPLC & HPLC & 13 \\
\hline phenanthridine & HPLC & HPLC & 45 \\
\hline m-cresol & Acid/Base Extraction & GC & 330 \\
\hline p-cresol & Acid/Base Extraction & GC & 270 \\
\hline 2,4-dimenthylphenol & Acid/Base Extraction & GC & 380 \\
\hline 2,5-dimethyl phenol & Acid/Base Extraction & GC & 320 \\
\hline $2,5,6$-trimethylphenol & HPLC & $\mathrm{GC} / \mathrm{MS}$ & 360 \\
\hline $2,4,6$-trimethylphenol & HPLC & GC/MS & 120 \\
\hline
\end{tabular}

1580, Organics in Shale 0il, is intended primarily for evaluating the reliability of analytical methods used for the determination of trace level polynuclear aromatic hydrocarbons, phenols, and nitrogen heterocyclic compounds in shale oil, coal-derived liquids, or petroleum products. In addition, this SRM may be used as a surrogate standard for any complex environmental extract containing classes of compounds for which the SRM is certified.

J. M. Brown-Thomas, S. N. Chesler, F. R. Guenther, H. S. Hertz, L. R. Hilpert, P. L. Konash, W. E. May, R. M. Parris, K. L. Richie, S. A. Wise

11. Synthesis, Isotopic and Purity Analyses, and Preparation of Solutions of Stable Isotope Labeled Marker Compounds for Priority Pollutant Analysis

A "Master Analytical Scheme" being developed for the Environmental Protection Agency by the Research Triangle Institute and Gulf South Research Institute is intended to allow qualitative and quantitative analys is of organic chemicals in water by gas chromatography/mass spectrometry. The "Master Analytical Scheme" requires the use of internal standards to be developed by NBS. The standards each consist of seven to eleven deuterated compounds in a solvent. Three different mixtures for "purgeable", "extractable", and "intractable" organic compounds, each at two concentration levels (ranging from $0.25 \mu \mathrm{g} / \mathrm{L}$ to $2500 \mu \mathrm{g} / \mathrm{L}$ per component), are needed. 
of the 25 deuterated compounds required, two, 2-naphthalenesulfonic acid- $d_{7}$ and dodecylphosphate- $d_{25}$, were synthesized at NBS because they were not commercially available. The reaction sequences used are as follows:

2-Naphthal enesul fonic Acid-d 7
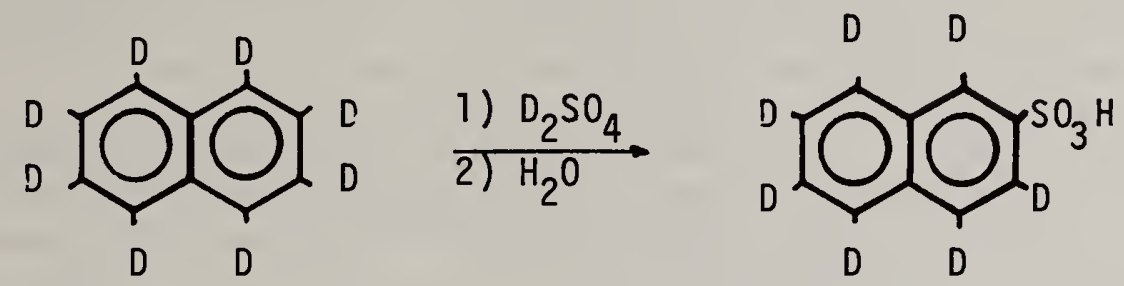

Dodecyl Phosphate- $d_{25}$

$$
\begin{aligned}
& \mathrm{CD}_{3}\left(\mathrm{CD}_{2}\right)_{11} \mathrm{OD} \stackrel{\left(\mathrm{C}_{6} \mathrm{H}_{5} \mathrm{O}\right)_{2} \mathrm{POCl}}{\text { pyridine }} \mathrm{CO}_{3}\left(\mathrm{CD}_{2}\right)_{11} \stackrel{\mathrm{OP}}{\mathrm{OP}}\left(\mathrm{OC}_{6} \mathrm{H}_{5}\right)_{2} \\
& \stackrel{\mathrm{H}_{2} / \mathrm{Pt}}{\mathrm{HOAC}} \mathrm{CD}_{3}\left(\mathrm{CD}_{2}\right)_{11} \stackrel{0}{\mathrm{OP}(\mathrm{OH})_{2}}
\end{aligned}
$$

A11 dehydrated compounds were checked for chemical purity by mass spectrometry and either gas or 1 iquid chromatography, and for isotopic purity by mass spectrometry.

Since some of the compounds were potentially unstable when stored in mixtures, several test mixtures similar to the internal standards, but of undeuterated compounds in deuterated solvents, were prepared. These were subjected to conditions to accelerate aging, and examined by nuclear magnetic resonance spectrometry. Although some decomposition was observed, the rates were such that properly stored mixtures are expected to be usable for the required several months of evaluation of the "Master Analytical Scheme".

Approximately 600 individual sealed vials of the various internal standard mixtures were prepared, and the 400 required for initial tests of the "Master Analytical Scheme" have been delivered. The remaining vials have been retained at NBS for stability tests, which are in progress.

S. N. Chesler, B. Coxon, P. Konash, W. E. May, R. M. Parris,

L. T. Sniegoski, M. J. Welch, E. White V 


\section{Evaluation of a Spectroelectrochemical System Using o-Tolidine}

A wide range of spectroscopic instrumentation has been used in conjunction with optically transparent electrodes in performing spectroelectrochemical experiments. The development of rapid-scanning spectrophotometers has permitted the entire spectrum of an absorbing species at an electrode to be obtained sufficiently fast to allow the extraction of useful kinetic and mechanistic information. To date, the rapid-scanning spectrophotometers used have been based on a motor-driven oscillating mirror. As an advance in this field, a silicon intensified target detector was selected as a state-of-the-art detector for our optical system. The light passing through the optically transparent thin-layer electrode is focused on the entrance slit of a grating polychromator. The dispersed spectrum falls on the vidicon, a matrix of discrete sensors which are interrogated electronically to generate spectral information. This type of system eliminates the problems associated with mechanically scanned spectra, and additionally, offers greater signal-to-noise ratios than rapid-scanning systems because of continuous integration of the signal by the vidicon.

After completion of the vidicon optical multichannel analyzer installation, the system was evaluated using the well-characterized compound, o-tolidine. A gold-minigrid optically transparent thin-layer electrode served as the working electrode in the spectroelectrochemical cell. A potentiostat was used to control the potential of the working electrode. In the thin-layer electrode, the potential applied to the working electrode determines the ratio of oxidized to reduced species for the entire solution. In the optically transparent cell, the concentration ratio is spectrally monitored for the absorbing species (figure 21). The formal reduction potential and stoichiometry of electron transfer of the absorbing species is precisely calculated from the spectral information obtained at various applied potentials. Values obtained for o-tolidine from solution concentrations ranging from $2.8 \mathrm{mmol} / \mathrm{L}$ to $0.01 \mathrm{mmol} / \mathrm{L}$ gave excellent agreement with 7 iterature data obtained by rapid-scanning spectrometers. The vidicon proved capable of detecting changes of less than 0.002 absorbance unit, showing it to be more sensitive than the rapid-scanning spectrometer.

The spectroelectrochemistry system will be used to study the redox properties of ceruloplasmin, a serum copper-transport protein. The thinlayer spectroelectrochemical technique is well suited to the study of biological molecules with redox centers surrounded by a protein matrix. Since such centers often exchange electrons poorly with an electrode, a mediator-titrant molecule which transports electrons between the electrode and biological molecule can be added to the test solution or bound to the electrode. Redox information can then be obtained by monitoring the chromophore spectrum of the protein.

M. L. Fultz 


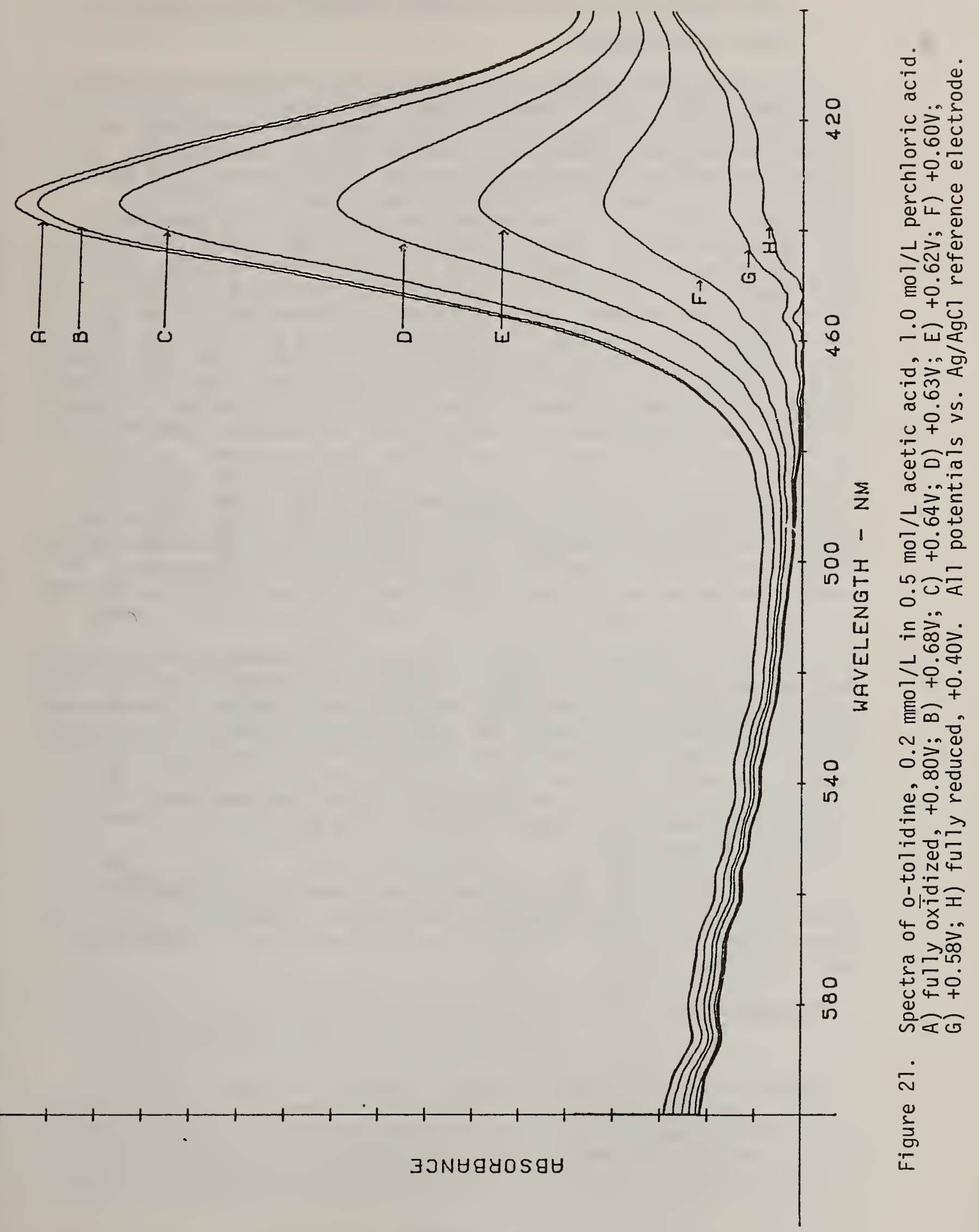


13. Development of a System for Computer-Controlled Rapid Electrochemical Measurements

Applications of rapid electrochemical measurements have increased from their original use in quantitative single-drop polarography to such scan-rate-sensitive areas as fast Fourier transform voltammetry and electrochemical detection in high-pressure liquid chromatography. A computer-controlled electrochemical system (CCES) enhances the implementation of such rapid electrochemical experiments by providing experimental flexibility through software control and by providing precise signal timing. The CCES consists of a $Z 80$ central processing unit, $64 \mathrm{~K}$ random access memory, and $240 \mathrm{~K}$ dual drive disk memory. An I/O interface has been built to connect the CCES with a commercial potentiostat and provides the digital/analog conversion for the rapid electrochemical experiments. Construction of the CCES has been completed and is undergoing testing in conjunction with a commercial potentiostat. The scan rates of the CCES are as fast as any digital system developed, capable of a staircase potential scan rate of 5 volts/second for a one millivolt step height and of 2 volts/second for a similar step height in the differential pulse mode. The CCES has been programmed to signal average in real time. This is acheived, however, at the expense of erasing the individual scan records and at a slight reduction in scan rate. We have also incorporated a graphics monitor that displays the response function with respect to the applied working electrode potential in real time. It is, therefore, possible to determine immediately whether the experiment is proceeding properly. Previously, the data had to be graphed on a rather slow plotter before it could be evaluated.

The rate at which the potential range of an electrochemical experiment can be scanned and still be an accurate representation of the applied potential is determined by the cell time constant. Because the time constant is determined by the series combination of uncompensated resistance between the working and reference electrodes and the doublelayer capacitance of the working electrode, we have constructed an electrochemical cell which minimizes the inter-electrode separation by the use of a pseudo-reference electrode and by using a working electrode of minimal surface area.

The CCES has a decided advantage over commercial el ectrochemical systems in its increased flexibility and speed. Combined with the lowtime-constant cell, the CCES should be a valuable tool in determining fast electrode kinetics.

L. M. Doane 
14. Development of a High-Sensitivity Dual-Electrode Detector for

Liquid Chromatography

Reductive electrochemical detection in liquid chromatography (LC) is potentialiy a very powerful measurement technique in the determination of trace organic pollutants by virtue of its sensitivity and selectivity. However, for the best sensitivity, oxygen exclusion is required. This makes the technique cumbersome to use and has restricted its application.

A new approach for reductive $L C$ detection that eliminates the need for complete oxygen exclusion has been developed. A two-electrode, thin-layer cell is used in a manner conceptually similar to a ring-disc system. Two closely spaced electrodes are poised at different potentials. The first electrode encountered by the flowing electrolyte is held at a potential sufficiently negative to reduce the analyte. The second detector electrode is held at a potential sufficiently positive to reoxidize the generated species and also to avoid the reduction of oxygen. The low residual current at this potential assures a low background noise, upon which the analytical current is easily measured with high sensitivity.

The best analytical response is obtained for analytes that either are reversibly reduced or that have chemical follow-up reactions that generate easily oxidized products. The reductive/oxidative behavior of three important industrial/environmental pollutants--nitrobenzene, dichlone, and tributyltin--have been studied by reverse pulse voltammetry.

The sensitivity and linearity of the dual electrode technique has been evaluated by comparison to direct reductive detection. Two chromatograms with equal concentrations are pictured in figure 22 . The baseline noise of direct reductive detection without use of an inert atmosphere box is apparent (upper figure). The lower chromatogram shows the very low baseline noise of the dual electrode approach achieved with a minimum of difficulty.

Current experiments involve the evaluation of the technique's selectivity by application to the measurement of organic analytes in environmental samples. Also, investigation is in progress on the use of modulated waveforms and phase selective detection to improve selectivity.

W. A. Maccrehan

15. Estimation of Thermodynamic and Kinetic Data from Spectroelectrochemical Studies Using Digital Simulations

Croconate compounds, a class of pseudo-oxocarbons, are interesting from both the electrochemical and spectroscopic points of view. These compounds, which are highly colored in their various forms, exhibit very rich electrochemical behavior during cyclic voltammetric studies. We have continued the study of the electrochemical reactions of the potassium 

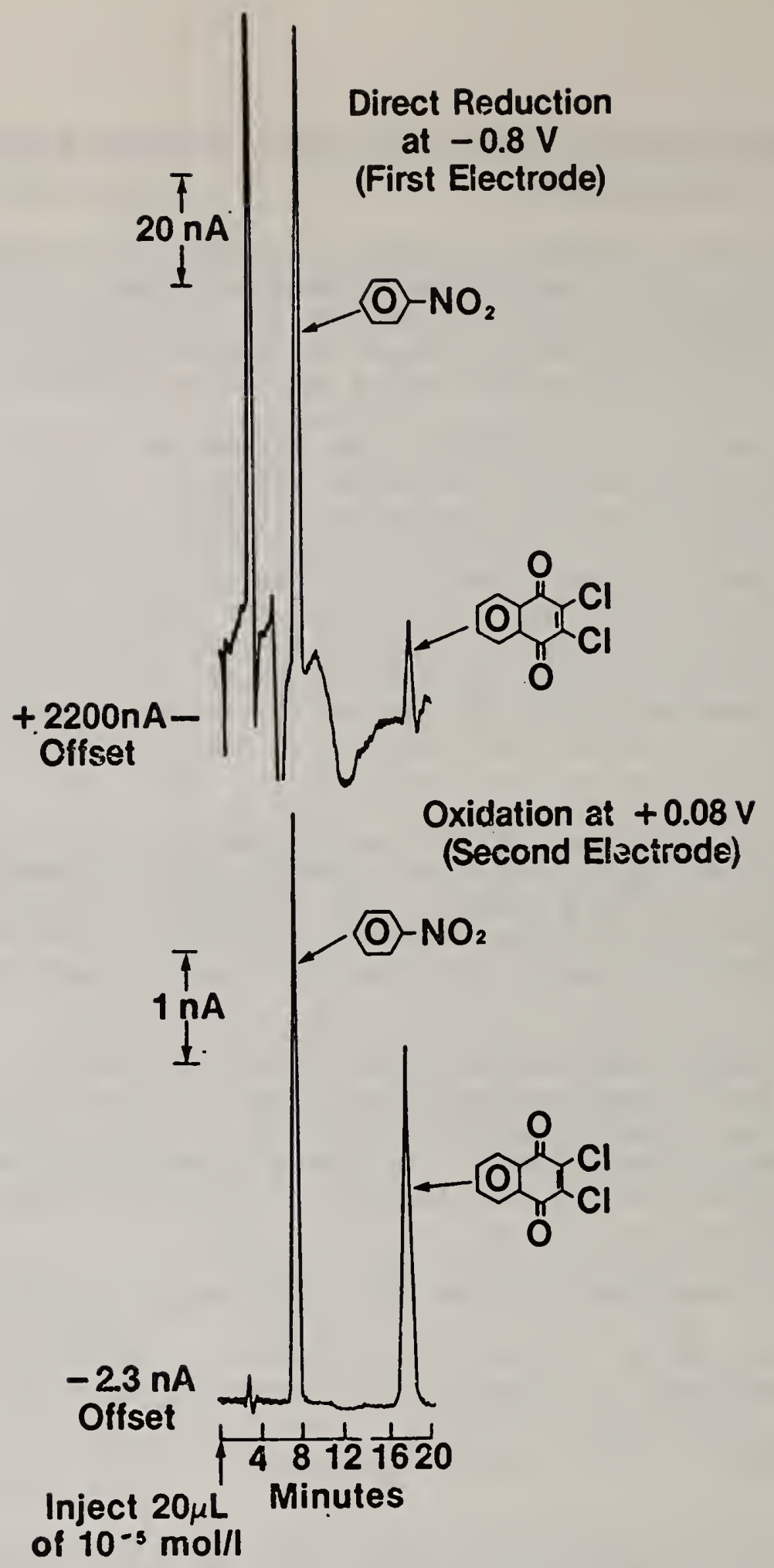

Figure 22. Different Reductive Detection Modes. Conditions: Analytes-$1.0 \times 10^{-5} \mathrm{~mol} / \mathrm{L}$ of nitrobenzene and dichlone; Solvent-- 60 percent $\mathrm{MeOH} / \mathrm{H}_{2} \mathrm{O}, 0.1 \mathrm{~mol} / \mathrm{L} \mathrm{NH} \mathrm{N}_{4} \mathrm{OAC} \mathrm{pH} 5.2$ at $1.0 \mathrm{~mL} / \mathrm{minute}$; Column--Whatman Partisil PXS $5 \mathrm{\mu m}$. Upper figure--direct reductive detection at $-0.8 \mathrm{~V}$ without enclosure of the LC in an inert atmosphere box. Lower figure--dual electrode detection, $-0.8 \mathrm{~V}$ on generator electrode, +0.08 V on detector electrode. 
salt of croconate violet. Cyclic voltammetry, done with rotating ringdisc electrodes, showed a reversible wave when scanned between $+0.2 \mathrm{~V}$ to $+0.8 \mathrm{~V}$, with respect to the SCE, on glassy carbon electrodes. The experimental cyclic voltammogram was fitted, by non-linear regression, with the theoretical curve for a simple reversible electrode reaction. This gives an $E^{\circ}$ of $+0.57 \mathrm{~V}$ and a diffusion coefficient of about $0.3 \times 10^{-5}$ $\mathrm{cm}^{2} /$ second. However, the experimental value for the separation of the cathodic and anodic peaks $(75 \mathrm{mV})$ is significantly larger than the theoretical value of $57 \mathrm{mV}$ for this case. We tried the simple reversible scheme first because of the relative ease of doing a regression calculation for this case and because the current can be expressed explicitly as a function of the potential. For more complex cases, we had to use numerical simulation to obtain theoretical curves. For instance, in the case of a chemical reaction following the electrode reaction, the peak separation and also the shift in the position of the peaks are shown to be dependent both on the equilibrium constant and on the ratio of the potential scan rate to the chemical reaction rate. We are now exploring the possibility of using these correlations in the determination of the mechanism and the kinetic parameters.

Preliminary experiments performed in the spectroelectrochemical thin-layer gold-minigrid cell showed only minor changes in the spectrum of croconate violet after the application of potentials ranging from $+0.8 \mathrm{~V}$ to $-0.3 \mathrm{~V}$, indicating differences in the redox and chromophore centers of the molecule. We intend to study this effect further.

Theoretical working curves for various electrochemical processes were computed since they are important aids in the interpretation and elucidation of the kinetics and mechanisms of the electrochemical reactions. For example, figure 23 shows the ratio of the charges transferred during the forward and backward steps in double potential step chronocoulometry as a function of the duration of Step 1 for different values of the rate constant for the homogeneous chemical reaction following the electrode reaction. Figure 24 illustrates the numerical simulation of the process

$$
0 x+n e^{-} \stackrel{R}{\gtrless} \text { ed } \stackrel{k}{\rightarrow} z
$$

in a thin-layer spectroelectrochemical cell. Here the logarithm of the absorbance is shown as a function of time for cases where the extinction coefficient of $Z$ is ten times that the reduced species. This simulation is needed to determine the feasibility of using the optically transparent cell to obtain kinetic information for electrochemical reactions (in contrast to the usual equilibrium case).

E. A. Blubaugh, Jr., M. L. Fultz,W. T. Yap 


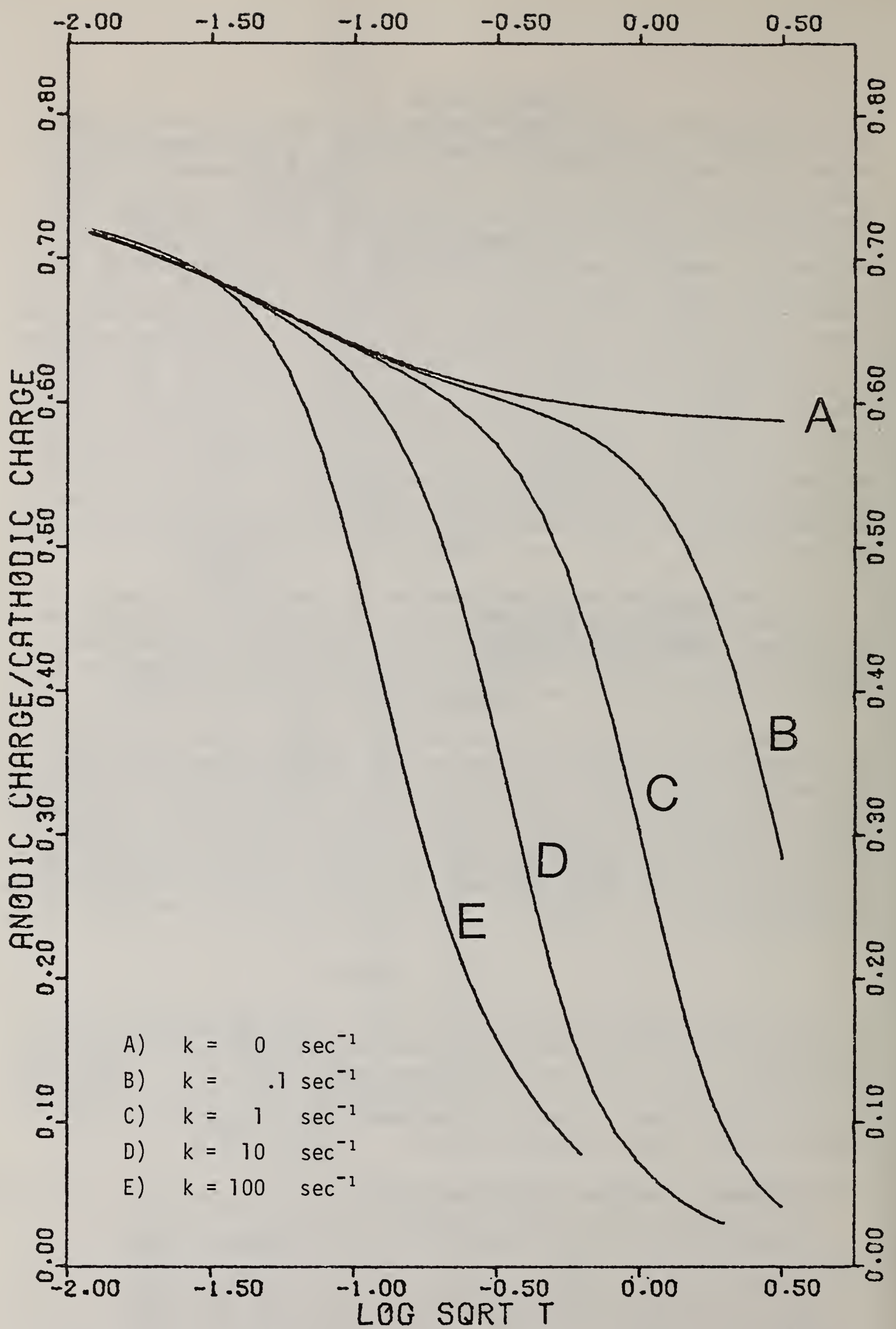

Figure 23. Theoretical working curves for the ratio of anodic charge to cathodic charge as a function of log square root of time. Where $A$ through $E$ are the working curves obtained for increasing magnitude of the rate constant. 


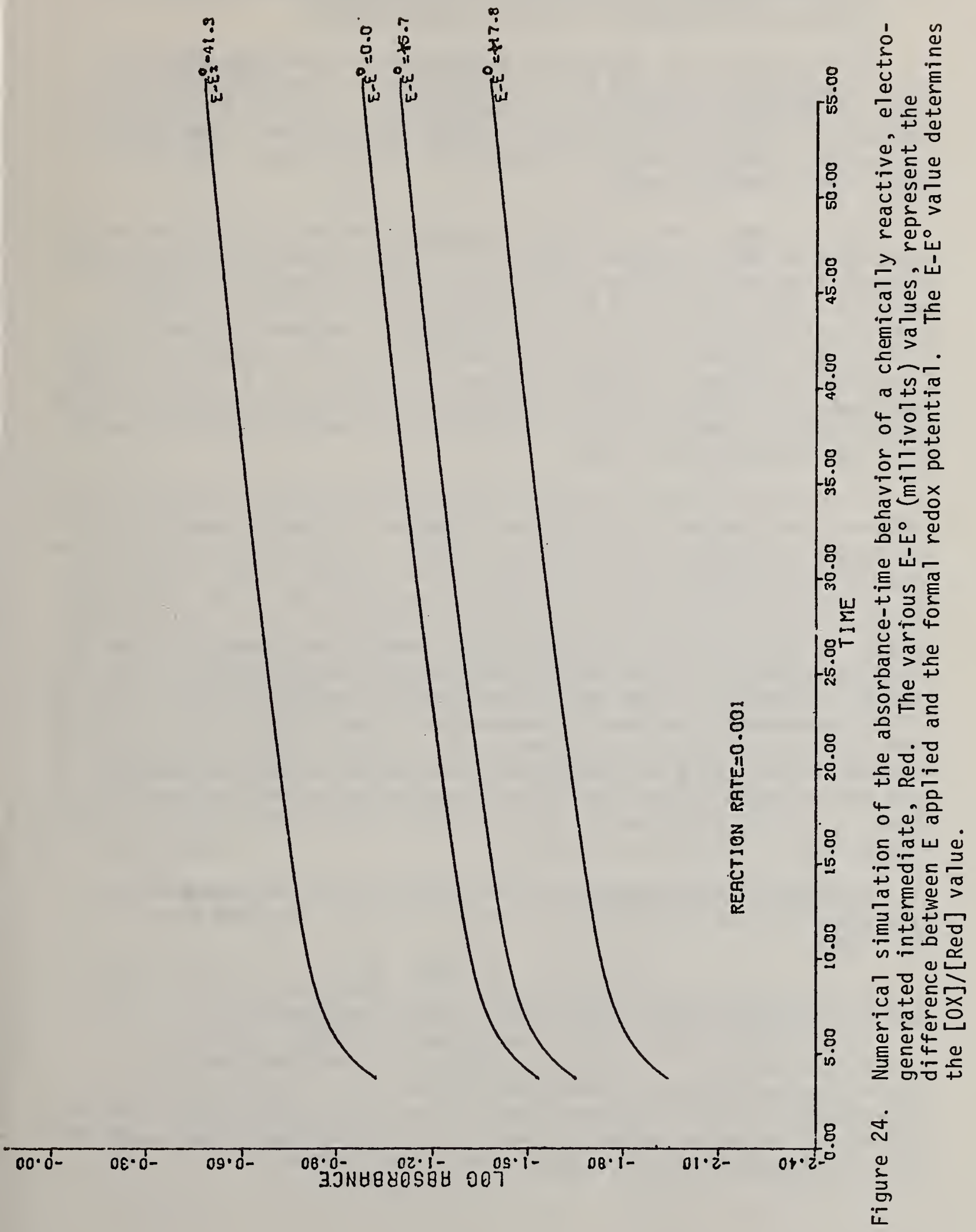


16. Method Developed for Determination of Ambient Temperature Vapor Pressures of Polynuclear Aromatic Hydrocarbons

The feasibility of developing a Standard Reference Material for organic compounds in air is being investigated. A gas-saturation technique is being used to produce a saturated vapor solution of an organic compound at its thermodynamic equilibrium vapor pressure. The vapor is cryogenically trapped and then analyzed by coupled-column high-performance liquid chromatography.

The gas saturation method for the determination of vapor pressures dates back to 1845. However, due to the lack of sufficiently sensitive analytical techniques, this method has not been extensively used until recently. M. M. Cliath and W. F. Spencer [1], in a series of experiments, utilized the method to study desorption isotherms of pesticides. P. Pella [2] coupled a gas-saturator to a gas dilution system to produce a calibration system for exploring vapor detectors. W. E. May [3] used the dynamic-coupled-column HPLC technique to determine the vapor pressure of naphthalene. Comparison with published data showed that a systematic error was present in this work.

This study has been concerned with eliminating the systematic errors discovered earlier and extending the technique to vapor-phase studies of the larger polynuclear aromatic hydrocarbons. The dynamic-coupled-column HPLC system has been modified to include dual 6 -port valves which allow for purging of the collection assembly by an inert gas. The techniques of column conditioning used for the water SRM generator columns have been applied to this work in order to properly remove any impurities from the starting material. The system is designed to permit continuous flow through all channels so as to eliminate sorption effects. Thus, the system is dynamic in nature. The generator columns and all connecting flow lines are constantly submerged in an isothermal bath. The vapor is concentrated on either an octadecyl silane-packed column (C-18) or via a cold trap assembly. The initial work has used an anthracene generator column. The concentration at $25^{\circ} \mathrm{C}$ has been determined as $60.2 \mathrm{ng} / \mathrm{L}$ with a precision of 1.4 percent relative.

Studies of the dependence of the flow rate upon the vapor density have shown that the vapor density remains constant for flow rates below $14 \mathrm{~cm}^{3} / \mathrm{min}$.

Initial temperature studies (Table 14 ) have shown excellent agreement with extrapolated data obtained by other techniques.

Future work will include studies of several columns containing varying concentrations of anthracene-coated $\mathrm{SiO}_{2}$. The effects due to the presence of other compounds and that due to the water vapor concentration will also be studied. Finally, a systematic check will also be used to determine if any systematic errors are present. 
Table 14. Preliminary Determination of the Anthracene

Vapor Pressures as a Function of Temperature.

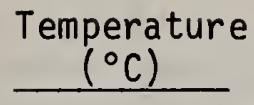

15.3
Temperature

$\left({ }^{\circ} \mathrm{K}\right)$

288.5

\section{Pressure torr $\times 10^{6}$}

2.68

2.57

2.75

20.4

25.0

298.2

6.39

6.19

30.1

303.3

11.4

11.6

11.1

34.9

308.1

20.3

19.9

19.4

As soon as the technique has been completely verified, it will be used to obtain the vapor pressure curves at ambient temperature for a series of polycyclic aromatic compounds. This data is presently available only by extrapolation of data obtained at higher temperatures.

Further applications will involve the use of the generator columns for calibration of high-volume atmospheric sampling systems.

\section{References}

[1] Spencer, W. F. and $\mathrm{Cl}$ iath, M. M. , Environ. Sci. Technol. 3 , 670 (1969).

[2] Pella, P. A., Anal. Chem. 48, 1632 (1976).

[3] May, W. E., Annual Report 1978, Center for Analytical Chemistry, National Measurement Laboratory, to NAS-NRC Evaluation Panel, 141 (1978).

W. E. May, W. J. Sonnefeld 


\section{Combined LC/MS Techniques for Direct Quantitative Analysis of Individual Organic Compounds in Complex Mixtures}

An interface for a conventional liquid chromatograph and a conventional mass spectrometer has been constructed which operates on the principle of pre-concentrating a liquid stream and then admitting part of it to the ionization region of the mass spectrometer. The selectivity of the mass spectrometer and the reproducible behavior of the entire system allow the direct quantitation of substances in complex mixtures.

The pre-concentration is accomplished by allowing the effluent from the liquid chromatograph to flow down a heated wire. The evaporation of solvent has yielded up to a 20-fold enrichment of analyte, and it is believed that 100-fold enrichment is feasible.

The flow into the mass spectrometer is controlled by a small needle valve built into the tip of the inlet tube. The valve is formed by a $0.05 \mathrm{~mm}$ teflon film at the tip of the tube pierced by a pointed $0.1 \mathrm{~mm}$ wire which passes through the bore of the tube. Flow rate into the mass spectrometer can be controlled in the range of 0-20 $\mu \mathrm{L} /$ minute. Since all of the pressure drop occurs at the high vacuum end of the tube, boiling within the tube with consequent unstable flow is avoided.

The stability of the system is such that quantitation can be done with external as well as internal standards. Figure 25 shows typical chromatograms from the determination of phenol in a shale oil matrix using external standards. The level found was $400 \pm 40 \mathrm{ppm}$, a value in good agreement with, and with precision comparable to, two other methods requiring lengthy sample pretreatment. The direct shale oil determination by LC/MS required about 15 minutes per run.

Preliminary experiments suggest that it will be possible to quantitate valproic acid, an anticonvulsant drug, in serum utilizing direct injections of serum in reversed-phase chromatography. Other methods of determining the levels of this drug (which is typically $100 \mathrm{ppm}$ ) involve extracting the drug and preparing derivatives, which are then analyzed by conventional liquid or gas chromatographic techniques.

R. G. Christensen, H. S. Hertz, S. Meiselman, E. White V

18. Correlation of Liquid Chromatographic Retention Data with Molecular Shapes for Polycyclic Aromatic Hydrocarbons

Reverse-phase liquid chromatography (LC) on chemically-bonded $C_{18}$ stationary phases is presently the most popular liquid chroamtographic separation mode. The mechanism of separation on these $C_{18}$ materials has not been established and is a topic of much discussion and research. The study of the reverse-phase retention characteristics of polycyclic aromatic hydrocarbons (PAH's) provides valuable information because (1) PAH's are ideal model compounds for investigating the mechanism of 


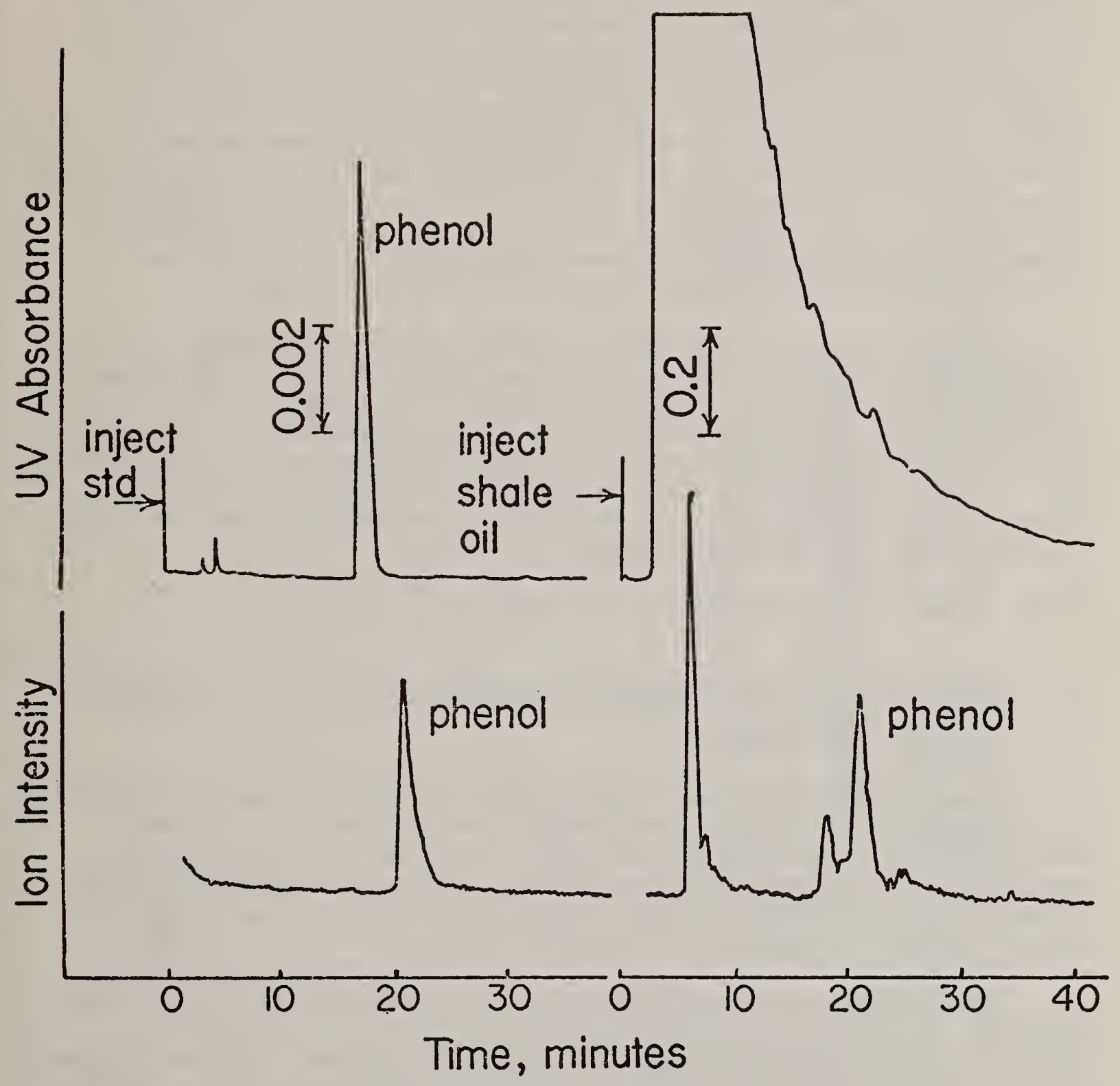

Figure 25. Chromatogram of shale oil obtained during phenol quantitation. Upper trace: ultraviolet detector recording at $254 \mathrm{~nm}$. Lower trace: single ion record at $\mathrm{m} / \mathrm{z} 94$. 
retention due to their nonpolarity, and (2) the separation and identification of PAH's are of environmental interest because of the production of $\mathrm{PAH}^{\prime}$ s during combustion of fuel and their suspected carcinogenic properties.

Reverse-phase $L C$ on $C_{18}$ phases provides unique selectivity for the separation of isomeric $\mathrm{PAH}^{\prime} \mathrm{s}$ and particularly methyl-substituted PAH's. A relationship was observed between the shape of PAH solutes, particularly the length-to-breadth ratios and the reverse-phase LC retention on $\mathrm{C}_{18}$ bonded phases. In nearly all cases, this ratio was successful in predicting the LC elution order of isomeric PAH's for more than 40 unsubstituted and 40 methyl-substituted PAH's, i.e., the retention increases with increasing length-to-breadth ratio. These ratios were found to be particularly useful in predicting the selectivity of reversephase LC for methyl-substituted PAH's. The linear correlation for the LC retention vs the length-to-breadth ratio for 23 methyl-substituted isomeric benzo[c]phenanthrenes, benz [a] anthracenes, and chrysenes is illustrated in figure 26 (correlation coefficient $=0.936$ ).

This relationship suggests the importance of solute shape in the LC mechanism. In GC, a similar relationship exists between shape and retention on liquid crystal phases, suggesting the possibility of a 1 iquid crystal effect or "ordering" of the $C_{18}$ phase in LC. The retention characteristics on polymeric $C_{18}$ phases exhibit greater selectivity and a greater correlation with the shape data than on the monomeric phases. This observation indicates that polymeric phases may be more "ordered" than the monomeric phases.

W. J. Bonnett, F. R. Guenther, W. E. May, S. A. Wise

\section{Application of Multi-Dimensional Chromatography to Trace Organic}

Compound Quantitation

A multi-dimensional gas chromatographic technique has been devised for the analysis of single compounds in complex matrices. A simple switching device was constructed which allows the transferring of a portion of the effluent from one capillary column to another capillary column. Under proper conditions, this transfer can be done without the use of cryogenic trapping on the second column.

This technique was used to certify 5,6-benzoquinoline in the shale oil SRM. An acid/base extraction scheme was used to isolate the $\mathrm{N}$-heterocyclic containing fractions. The fraction was then concentrated under a stream of dry nitrogen to approximately $100 \mu \mathrm{L}$. Two $\mu \mathrm{L}$ was then injected onto the first column, a non-polar SE52 capillary column, to give a boiling point separation. Subsequently, a portion of the effluent containing the compound of interest was transferred from the first column to a carbowax 20M capillary column. Separation on this column is based on polarity. The analyte was easily separated from all other compounds. A standard addition technique was used with phenanthridine as an internal volume corrector. This procedure was used on eight separate groups of ampoules from the SRM lot. The final concentration was determined to be 


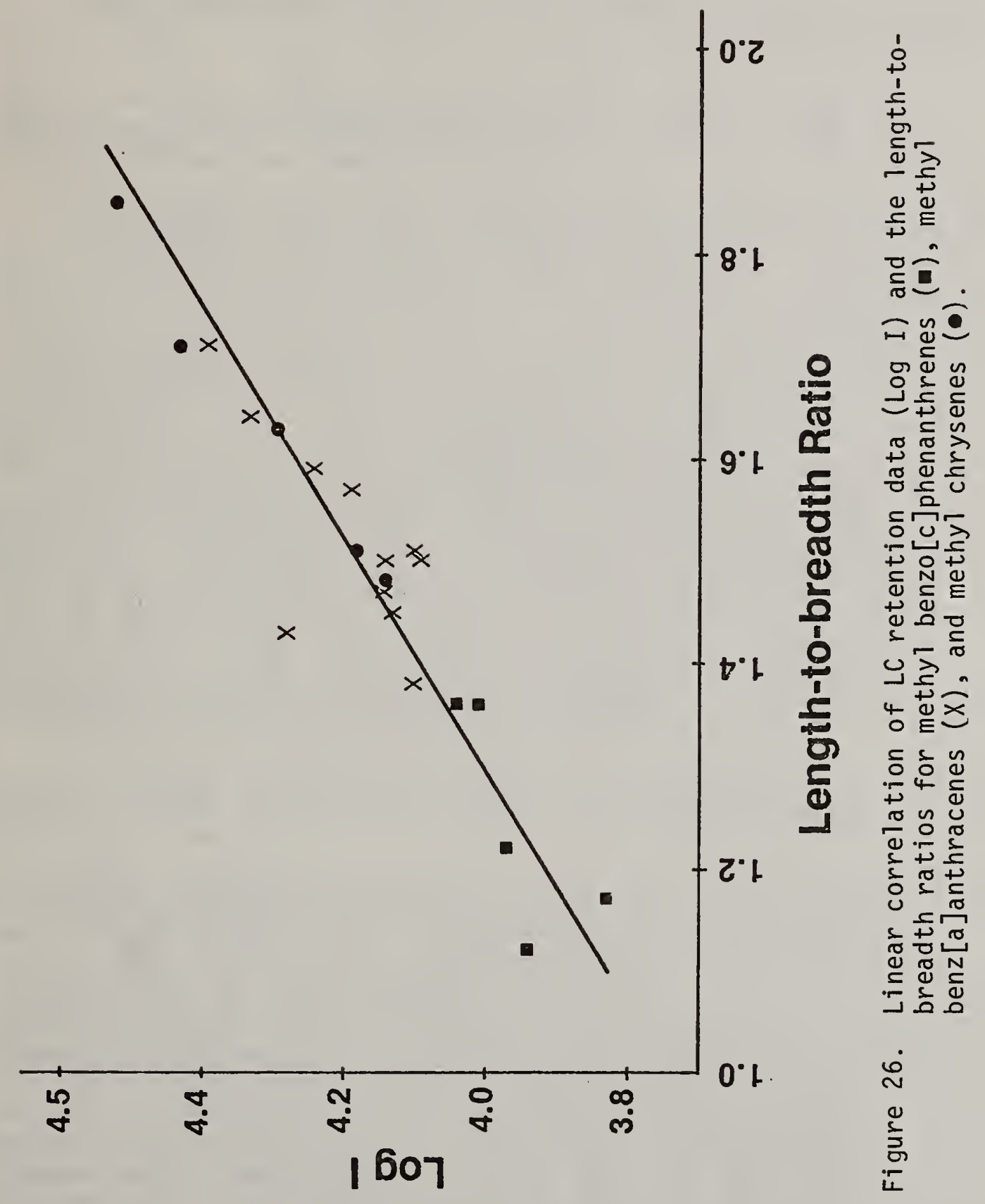


$15.1 \pm 1.2 \mathrm{ppm}$ (one standard deviation). This compares favorably with the liquid chromatographic determination of $16.3 \pm 1.8 \mathrm{ppm}$.

S. N. Chesler, F. R. Guenther

20. Development of a Procedure for the Analysis of Polychlorinated Biphenyls in Recycled 0il

Polychlorinated biphenyl (PCB) mixtures (Aroclors) have been extensively used in this country in high-voltage electrical components. These toxic compounds find their way into the environment when the electrical components containing them are discarded. The Aroclor fluid physically resembles lubricating oil and many instances have been reported where toxic PCB's have been mistakenly added to oils being collected for recycling purposes. It has, therefore, been necessary to develop an analytical procedure for the determination of PCB's in waste oils. PCB's are organic compounds which possess no simple chemical characteristics which will allow them to be easily separated from a hydrocarbon matrix such as found in lubricants. Direct determination of PCB's in waste oils using electron capture gas chromatographic methods is not feasible due to the large number of electron-capturing organic impurities present in this matrix; e.g., sulfur and oxygen containing compounds, and polynuclear aromatic hydrocarbons. Solvent extraction and normal column chromatography were found ineffective for the removal of these interferences. We, therefore, developed a HPLC cleanup technique which removes the major interfering components. A small amount $(140 \mu \mathrm{L})$ of $0 i 1$ is injected onto an aminosilane preparatory scale LC column and an appropriate volume fraction of the eluate, containing the PCB's, is collected. This fraction has been shown to contain very few organic compounds which acted as analytical interferences. The PCB's are then quantitated using an SE-52 WCOT (nonpolar) column. If the sample is found to contain more than one PCB mixture, the ratio of mixtures is determined by a matrix algebra technique using a newly developed computer program.

The presence of PCB's at a level of $10 \mu \mathrm{g} / \mathrm{g}$ has been verified in a number of used and recycled oil samples obtained for testing the methodology. The type(s) of PCB mixtures present in these samples were successfully identified using standard retention data and visual pattern recognition techniques. Mixture ratios were determined to \pm 20 percent using the computer program. Since the electron capture detector normally used in PCB analyses exhibits large variations in response factors toward different PCB compounds, accurate quantitation has been found to be difficult. A different type of detector is now being employed. This detector ( $\mathrm{Ha} l 1$ detector) operates by measuring the amount of $\mathrm{HCl}$ released from the PCB's when they are reacted at high temperatures in the presence of $\mathrm{H}_{2}$ gas. The Hall detector does not have the limitation of the electron capture detector and simple calibration techniques can be used.

S. N. Chesler, F. R. Guenther, R. M. Parris, P. A. White 
21. Development of a Fourier Transform Infrared Spectrometer-Gas Chromatograph Interface and Its Application to Pollutant Identification

Installation of a Nicolet 7000 Fourier transform infrared (FTIR) spectrometer was begun in September 1979, and essentially completed by January 1980. The instrument was found to meet all design specifications and even exceeded some, such as resolution and signal-to-noise ratio. The instrument is being used for four distinct research and/or service functions: high-resolution low-pressure gas work, liquid samples, liquid chromatography, and gas chromatography.

The studies of gas chromatographic applications have progressed through the utilization of packed column chromatography to the construction and testing of an interface for wall coated open tubular gas chromatographic columns (WCOT), which will allow real-time FTIR analys is of compounds eluting from the WCOT. The use of packed columns was not pursued because they were not found to have the resolving power required for the separation of the complex samples we wished to analyze. The WCOT columns used in this project were chosen to maximize sample capacity of the column, since the FTIR instrument requires $\sim 100 \mathrm{ng}$ of component for the collection of a reasonably identifiable spectrum. Also, an on-column injector was fabricated to facilitate injection of a maximum amount of sample onto the column without undo loss of efficiency. The first interface design consisted of a Pt/Ir tube which connected the output of the thermal conductivity detector (TCD) to the FTIR light pipe. This interface was found to be unsuitable because too much dead volume (located mostly in the TCD and connecting fitting) caused unacceptable peak broadening. In addition, the $\mathrm{Pt} / \mathrm{Ir}$ tube thermally trapped out and/or reacted with all solutes boiling above $300^{\circ} \mathrm{C}$.

The present interface consists of a $50 \mathrm{~cm}$ length of deactivated, methyl silicone-coated quartz capillary of $0.15 \mathrm{~mm}$ internal diameter. The TCD has been bypassed and no other gas chromatographic detectors are used. This interface is connected to a glass WCOT by use of $\mathrm{AgCl}$ cement; if a quartz WCOT were used, then the last $50 \mathrm{~cm}$ of the capillary itself could act as the interface. The quartz interface does not appear to react with or retard eluting compounds and spectra have been obtained for compounds such as methyl dibenzanthracene (b.p. $<500^{\circ} \mathrm{C}$ ). Reconstructed gas chromatograms (from IR data) still show large losses in efficiency over those obtained from normal gas chromatographic detectors. This loss is most likely due to the large volume of the FTIR light pipe, and a new low volume light pipe is being procured. Even though the FTIR data does not fully reflect the high-efficiency separations obtained on the WCOT columns, very recognizable and identifiable spectra are being obtained from environmental samples being analyzed (e.g., phenols in shale oil, PCB's, etc.). This is, to a large extent, being made possible by some newly developed manipulative computer software which numerically regains some of this lost column efficiency.

S. N. Chesler, F. R. Guenther 
C. Outputs and Interactions

(Organic Analytical Research Division)

1. Publications

Coxon, B. and Hough, L., Revision, by Proton, Carbon-13, and Nitrogen-15 NMR Spectroscopy, of the Structure Assigned a Bis (hexopyranosyl)amine Derivative, Carbohydr. Res. 73, 47-57 (1979).

Durst, R. A., Container Materials for the Preservation of Trace Substances in Environmental Specimens, in Monitoring Environmental Materials and Specimen Banking, N. P. Luepke, ed., Martinus Nyhoff Publishers, Boston, MA, 198-202 (1979).

Hertz, H. S., Certification of Clinical and Environmental Trace Organic Research Materials, Proc. Inter. Sym. on the Prod. and Use of Res. Matls., West Berlin (1979).

Maccrehan, W. A. and Durst, R. A., Measurement of Organometal Species in Biological and Water Samples Using Liquid Chromatography with Electrochemical Detection, Interagency Energy/Environment R\&D Progress Report, EPA-600/7-79-211, 63 pages (1979).

Margolis, S. A. and Schaffer, R., Development of a Standard Reference Material for Angiotensin I, Final Report on Agreement \#1-YOI-HU70042-06 (1979).

Mighel1, A. D., Hubbard, C. R., and Cohen, A., 2-Ethoxy-1,7,9-Triethy1-7, 9-Dihydro-1H-Purine-6,8-Dione, a Tetraethyl Derivative of Uric Acid, Acta Cryst, B35, 1280-1282 (1979).

Reeder, D. J., Place of Reference Materials and Reference Methods in the Evaluation of Drug Effects, Proc. of Workshop Under the Aegis of the Comm. of Med. Res. and Public Health of the Comm. of Eur. Communities (1979).

Sweeting, L. M., Coxon, B., and Varma, R., Conformational Analysis of Peracetylated Hexononitriles, Carbohydr. Res. 72, 43-55 (1979).

Velapoldi, R. A., Paule, R. C., Schaffer, R., Mandel, J., Murphy, T. J., and Gramlich, J. W., Standard Reference Materials: A Reference Material for the Determination of Chloride in Serum, NBS Special Publication 260-67 (1979).

Wise, S. A., Mowery, R. A., Jr., and Juvet, R. S., Jr., A Universal Detector for Liquid Chromatography: The Flame Aerosol Detector, J. Chromatogr. Sci. 17, 601 (1979).

Bowers, G. N., Jr., McComb, R. B., Christensen, R. G., and Schaffer, R., High-Purity 4-Nitrophenol: Purification, Characterization, and Specifications for Use as a Spectrophotometric Reference Material, Clin. Chem. 26 (6), 724-729 (1980). 
Chesler, S. N., Guenther, F. R., and Christensen, R. G., An Electrically Heated Sample/Injector for Use with High-Efficiency Gas Chromatographic Columns, J. High Res. Chrom. and Chrom. Comm., 351-352 (1980).

Cohen, A., Hertz, H. S., Mandel, J., Paule, R. C., Schaffer, R., Sniegoski, L. T., Sun, T., Welch, M. J., and White V, E., Total Serum Cholesterol by Isotope Dilution/Mass Spectrometry: A Candidate Definitive Method, Cl in. Chem. 26 (7), 854-860 (1980).

Coxon, B., Carbon-13 Nuclear Magnetic Resonance Spectroscopy of FoodRelated Disaccharides and Trisaccharides, Developments in Food Carbohydrate-2, C. K. Lee, ed., Applied Science Publishers Ltd., Barking, England, 351-390 (1980).

Coxon, B., Fatiadi, A. J., Cohen, A., Hertz, H. S., and Schaffer, R., Nitrogen-15 NMR Evidence for the Structures of N-9H-Xanthen-9-yland N, N'-Di-9H-Xanthen-9-yl-Ureas, Org. Magnetic Reson. 13, 187-192 (1980).

Coxon, B. and Reynolds, R. C., The Synthes is and NMR Spectroscopy of Derivatives of 6-Amino-6-deoxy-D-galactose- $\underline{6}-{ }^{15} \underline{\mathrm{N}}$, Carbohydr. Res. 78, 1-16 (1980).

Farrington, J. W., Albaiges, J., Burns, K. A., Dunn, B. P., Eaton, P., Laseter, J. L., Parker, P. L., and Wise, S. A., Fossil Fuels, The International Mussel Watch, National Academy of Sciences, Washington, DC, 7-77 (1980).

Fatiadi, A. J., Infrared Spectra of Pectic Substances (Book Review), Carbohydr. Res. 80, C25-C26 (1980).

Fatiadi, A. J., Pseudo-0xocarbons. Synthes is of 2,1,3-Bis-, and 1,2,3Tris (Dicyanomethylene) Croconate Salts. New Bond-Delocalized Dianions, "Croconate Violet" and "Croconate Blue", J. Res. Nat. Bur. Std. 85, 73-86 (1980).

Fatiadi, A. J., Pseudo-0xocarbons. Synthes is of 1,2,3-Tris (Dicyanomethylene) Croconate Salts. A New Bond-Delocalized Dianion, Croconate Blue, J. Org. Chem. 45, 1338-9 (1980).

Himes, V. L., Mighell, A. G., Hubbard, C. R., and Fatiadi, A. J., New Bond-Delocalized Dianions: The Crystal Structure of 1,3-Bis (Dicyanomethylene) Croconate Salt, $\left(\mathrm{C}_{11} \mathrm{~N}_{4} \mathrm{O}_{3} \mathrm{~K}_{2} \cdot 2 \mathrm{H}_{2} \mathrm{O}\right)$, J. Res. Nat. Bur. Std. 85, 87-97 (1980).

Howe11, B. F., Kinetic Methods for Detecting Inhibitors in NADH and NADH-Dependent Enzymes, Methods in Enzymology 66, 55-62 (1980).

Hubbard, C. R., Mighell, A. D., and Fatiadi, A. J., Dixanthylurea (N,N'-di-9H-Xanthen-9-y7urea), J. Res. Nat. Bur. Std. 85, 205-210 (1980). 
Margolis, S. A. and Konash, P. J., Non-Peptide Impurities in Angiotensin I and Other Commercial Peptides, J. of High Res. Chrom. and Chrom. Comm. 3, 317-318 (1980).

May, W. E., The Solubility Behavior of Polycyclic Aromatic Hydrocarbons in Aqueous Systems, ACS Advances in Chem. Series 185 (7), 143-192 $(1980)$.

Wise, S. A., Chesler, S. N., Hertz, H. S., May, W. E., Guenther, F. R., and Hilpert, L. R., Determination of Trace Level Hydrocarbons in Marine Biota, Anal. Tech. in Environ. Chem., J. Albaiges, ed., Pergamon Press, 41-51 (1980).

Velapoldi, R., Paule, R., Schaffer, R., Mandel, J., Machlan, L., Garner, E., and Rains, T., A Reference Method for the Determination of Lithium in Serum, NBS Special Publication 260-69 (1980).

Brown, J. M., Wise, S. A., and May, W. E., Determination of Benzo(a)pyrene in Recycled 0ils by a Sequential HPLC Method, Inter. J. of Environ. Ana1. Chem., in press.

Chesler, S. N., May, W. E., White, P. A., Parris, R. M., and Guenther, F. R., Determination of Polychlorinated Biphenyls in Waste and Lubricating 0i1s, Proc. of Joint Conf. on Meas. and Stds. for Recycled 0il/Systems Perfor. and Durability, to be pubi ished as an NBS Special Publication (1980).

Christensen, R. G., Hertz, H. S., Meiselman, S., and White V, E., A Liquid Chromatograph/Mass Spectrometer Interface Incorporating Continuous Sample Preconcentration, Ana 1. Chem., submitted for publication.

Durst, R. A. and Bates, R. G., Hydrogen Ion Activity, Encyclopedia of Chem. Tech., third edition, John Wiley \& Sons, New York, in press.

Hertz, H. S., Brown, J. M., Chesler, S. N., Guenther, F. R., Hilpert, L. R., May, W. E., Parris, R. M., and Wise, S. A., Determination of Individual Organic Compounds in Shale 0i1, Anal. Chem., in press.

Hodgeson, J. A. and Fatiadi, A. J., A Survey of Research Needs on Personal Samplers for Toxic Organic Compounds, to be published as an NBS Special Publication (1980).

Kaplan, A. E., Weiss, E. R., Byrne, S. T., El-Torkey, N. M., and Margolis, S. A., The Effect of Purified NADH on the Activity of Lactate Dehydrogenase from Control and Chemically Transformed Cells, Nature, in press.

MacCrehan, W. A., Advantages of Differential Pulse Detection in Liquid Chromatography and the Application to the Measurement of Organometal Cations, Anal. Chem., submitted for publication. 
Schaffer, R., Velapoldi, R. A., Paule, R. C., Mandel, J., Bowers, G. N., Jr., Copeland, B. E., Rodgerson, D. 0., and White, J. C., Reference Method for Determination of Serum Sodium, Clin. Chem., in press.

Wise, S. A., Bonnett, W. J., and May, W. E., Normal- and Reverse-Phase Liquid Chromatographic Separations of Polycyclic Aromatic Hydrocarbons, Polynuclear Aromatic Hydrocarbons: Chemistry and Biological Effects, A. Bjorseth and A. J. Dennis, eds., Battelle Press, Columbus, $\mathrm{OH}$, in press.

Wise, S. A., Chesler, S. N., Guenther, F. R., Hertz, H. S., Hilpert, L. R., May, W. E., and Parris, R. M., Interlaboratory Comparison of Determinations of Trace Level Hydrocarbons in Mussels, Anal. Chem., in press.

2. Talks

A. J. Fatiadi, "Synthesis of 1,3(Dicyanomethylene)Croconate Salts. New Bond-Delocalized Dianions: Croconate Violet and Croconate Blue," National Meeting of the American Chemical Society, Washington Hilton, Washington, DC, Sept. 12, 1979.

B. Coxon, "Synthesis of Nitrogen-15 Labeled Amino Sugar Derivatives by Addition of Phthalimide- ${ }^{15} \mathrm{~N}$ to Carbohydrate Epoxides," 178th National Meeting of the American Chemical Society, Capitol Hilton, Washington, DC, Sept. 13, 1979.

E. White V, "Organic Isotope Dilution Mass Spectrometry for the Definitive Measurement of Serum Constituents," 6th Annual Meeting of the Federation of Analytical Chemistry and Spectroscopy Societies (FACSS), Philadelphia, PA, Sept. 17, 1979.

H. S. Hertz, "Standard Reference Materials for Clinical Analysis," 6th Annual Meeting of the Federation of Analytical Chemistry and Spectroscopy Societies (FACSS), Philadelphia, PA., Sept. 25, 1979.

S. A. Wise, "Normal and Reverse Phase Liquid Chromatographic Separations of Polycyclic Aromatic Hydrocarbons," 4th International Symposium on Polynuclear Aromatic Hydrocarbons, Battelle Columbus Laboratories, Columbus, $\mathrm{OH}$, Oct. 4, 1979.

B. Coxon, "Nitrogen-15 NMR Spectroscopy of Amino Sugars," 31 st Southeastern Regional Meeting of the American Chemical Society, Virginia Blue Ridge Section, Roanoke, VA, Oct. 24, 1979.

R. A. Durst, "Sources of Error in Using Ion-Selective Electrodes," EXPOCHEM 199, Astroha11, Houston, TX, Oct. 25, 1979.

H. S. Hertz, "Certification of Clinical and Environmental Trace Organic RMs," International Symposium on the Production and Use of Research Materials, Bundesanstalt für Materialprüfung, Berlin, West Germany, Nov. 16, 1979. 
D. J. Reeder, "Place of Reference Material and Reference Methods in the Evaluation of Drug Effects," Workshop Under the Aegis of the Committee of Medical Research and Public Health (CRM) of the Commission of European Communities, Abbaye des Premontres, Pont-A-Mousson, France, Dec. 17, 1979.

H. S. Hertz, "Chromatography and Mass Spectrometry: A Love-Hate Relationship?" Washington Area Chromatography Discussion Group, HewlettPackard, Rockvi11e, MD, Dec. 20, 1979.

H. S. Hertz, "Quantitative Aspects of Trace Organic Analys is," Knoxville College Chemistry Department Seminar, Knoxville, IN, jan. 16, 1980 .

W. E. May, "Quality Assurance in Trace Organic Analysis," Staff Seminar, Waters Associates, Milford, MA, Feb. 11, 1980.

W. E. May, "The Development of a PAH/Water SRM," Environmental Protection Agency, Cincinnati, OH, Mar. 5, 1980.

W. E. May, "Quantitative Analysis of Trace Organics in Shale $0 i 1$ by Sequential Liquid Chromatographic Analyses," 1980 Pittsburgh Conference on Analytical Chemistry and Applied Spectroscopy. Atlantic City, NJ, Mar. 11, 1980.

W. E. May, "Use of Fluorescence in HPLC Analysis," 1980 Pittsburgh Conference on Analytical Chemistry and Applied Spectroscopy, Atlantic City, NJ, Mar. 12, 1980.

H. S. Hertz, "Standard Reference Materials for Trace Organic Environmental Analysis," 1980 Pittsburgh Conference on Analytical Chemistry and Applied Spectroscopy, Atlantic City, NJ, Mar. 13, 1980.

B. Coxon, "Carbon-13 NMR Spectroscopy of Pyruvate Dimers," 179th National Meeting of the American Chemical Society, Houston, TX, Mar. 24, 1980.

W. E. May, "Interlaboratory Comparison of Environmental Analyses Associated with Synfuel Production," 179th National Meeting of the American Chemical Society, Houston, TX, Mar. 25, 1980.

W. E. May, "Use of High Performance Liquid Chromatography in Trace Organic Analysis," Chemistry Dept., University of Arkansas at Pine Bluff, Pine Bluff, AR, Mar. 28, 1980.

E. White $V$ and M. J. Welch, "The Accurate Determination of Organic Serum Constituents by Isotope Dilution GCMS or Making Sense of a Bloody Mess," Washington Area Mass Spectrometry Discussion Group, Hewlett Packard, Rockvil1e, MD, Apr. 7, 1980.

S. A. Wise, "High Performance Liquid Chromatographic Separation of Polycyclic Aromatic Hydrocarbons," Analytical Chemistry Division Seminar, University of Maryland, College Park, MD, Apr. 18, 1980. 
D. J. Reeder, "Spectrometric Studies of Alkaline Hydrolysis of Croconate Blue Salts," 14th Middle Atlantic Regional Meeting. American Chemical Society, King of Prussia, PA, Apr. 23, 1980.

L. R. Hilpert, "Mass Spectrometric Methods for the Quantitative Analys is of Trace Organic Constituents in Alternate Fuels, "American Society for Mass Spectrometry 28th Annual Conference, New York, NY, May 26, 1980.

M. J. Welch, "An Isotope Dilution Mass Spectrometric Method for the Accurate Determination of Urea in Human Serum, "American Society for Mass Spectrometry 28th Annual Conference, New York, NY, May 26, 1980.

K. L. Richie, "GC-MS Quantitation of Several Nitrogen Heterocycles in Solvent Refined Coal, "American Society for Mass Spectrometry 28th Annual Conference, New York, NY, May 28, 1980.

S. A. Margolis, "Separation of Angiotensin I \& II Analogs by Reverse Phase High Performance Liquid Chromatography, "American Society of Bio Chemists, New Orleans, LA, June 4, 1980.

R. E. Botto, "Intramolecular Hydrogen-Bond Energies Derived from N-15 Spin Lattice Relaxation Measurements," Gordon Research Conference on Physical Organic Chemistry, Brewster Academy, Wolfeboro, NH, June $9,1980$.

R. A. Durst, "Current Status of the $\mathrm{pH}$ Scale," International Federation of Clinical Chemistry Expert Panel on $\mathrm{pH}$ and Blood Gases, Herlev Hospita 1, Copenhagen, Denmark, June 16, 1980.

R. A. Durst, "Reference Materials for Blood pH and Gases," International Federation of Clinical Chemistry Expert Panel on $\mathrm{pH}$ and Blood Gases, Herlev Hospital, Copenhagen, Denmark, June 17, 1980.

S. A. Wise, "Chemical Characterization of Organic Compounds by HighPerformance Liquid Chromatography," 2nd Symposium on Environmental Analytical Chemistry, Brigham Young University, Provo, UT, June 18, 1980.

R. A. Durst, "NBS pH Scale Recommendations - Single vs. Multiple Primary pH Standards," IUPAC Conference on the Harmonization of pH Scale Recommendations, Calouste Gulbenkian Centre, Lisbon, Portugal, June $19,1980$.

R. A. Durst, "International Practical pH Scale," IUPAC Conference on the Harmonization of pH Scale Recommendations," Calouste Gulbenkian Centre, Lisbon, Portugal, June 19, 1980.

R. Schaffer, "SRM's and Definitive Methods for Providing the Accuracy Basis for Clinical Chemistry," Joint Meeting of the American Association for Clinical Chemistry and Canadian Society of Clinical Chemists, Boston, MA, July 24, 1980. 
S. A. Wise, "A Relationship Between Liquid Chromatographic Retention and the Shape of Polycyclic Aromatic Hydrocarbons," Gordon Research Conference on Analytical Chemistry, New Hampton School, New Hampton, $\mathrm{NH}$, Aug 11, 1980.

B. Coxon, "Analysis of Pyruvate Solutions by Proton NMR Spectroscopy at $400 \mathrm{MHz}$," Second Chemical Congress of the North American Continent, Las Vegas, NV, Aug 25, 1980.

B. Coxon, "Characterization of Boat Conformations of $\alpha-D-A]$ tropyranoside Derivatives by Proton NMR Spectroscopy at $400 \mathrm{MHz}$, $\bar{\pi}$ Second Chemical Congress of the North American Continent, Las Vegas, NV, Aug 25 , 1980.

W. A. MacCrehan, "Liquid Chromatography Using Dual Electrode Reductive Electrochemical Detection," Second Chemical Congress of the North American Continent, Las Vegas, NV, Aug. 25, 1980.

H. S. Hertz, "Development in the Analysis of Priority Pollutants by Liquid Chromatography-Mass Spectrometry and by Immunoassay Procedures," Second Chemical Congress of the North American Continent, Las Vegas, NV, Aug. 27, 1980.

M. J. Welch, "Isotope Dilution/Mass Spectrometric Methods for Accurate Determinations of Organic Serum Constituents," Second Chemical Congress of the North American Continent, Las Vegas, NV, Aug 28, 1980.

S. A. Margolis, "Nutrient Analysis Standards," Interagency Technical Committee (IATC) Working Group on Nutrient Composition of Foods, National Institutes of Health, Bethesda, MD, Sept. 10, 1980.

W. E. May, "Quality Assurance in Environmental Trace Organic Analysis," Chemistry Dept., University of Maryland, College Park, MD, Sept. 12, 1980.

S. A. Wise, "The Pilot Environmental Specimen Bank Program," EPA Narragansett, Narragansett, RI, Sept. 15, 1980.

3. Committee Assignments

Harry S. Hertz

Chairman, ASMS, Committee on Education

Member, ASTM, Committee DI 9 on Water

Member, CEQ, Interagency Task Force on Environmental Data and Monitoring Air Polution Data and Monitoring Working Group, Task Group in Anticipatory Monitoring

Board of Directors, NCCLS

Member, Program Advisory Committee, ACS, Division of Analytical Chemistry 
Bruce Coxon

Secretary, ASTM, Subcommittee E13.07 on Nuclear Magnetic Resonance, Committee El3 on Molecular Spectroscopy

Associate Referee, International Commission for Uniform Methods of Sugar Analysis, Subcommittee 5 on $100^{\circ} \mathrm{S}$ Point of Sugar Scale Referee, U.S. National Committee on Sugar Analysis, Subcommittee 5 on $100{ }^{\circ} \mathrm{S}$ Point of Sugar Scale

Richard A. Durst

Member, ASTM, Committee D19 on Water

Member, Department of the Interior, Office of Water Data Coordination, Task Group 5 on Chemical and Physical Quality of Water and Sediments

Secretary, IFCC, Scientific Committee, Analytical Section, Expert Panel on $\mathrm{pH}$ and Blood Gases

Member, IUPAC, Committee V on Analytical Chemistry, Subcommittee 5 on Electroanalytical Chemistry

Member, NCCLS, Subcommittee on Electroanalytical Methods

Member, NCCLS, Subcommittee on pH and Blood Gases

Member, SAMA, Instrument Section, Electroanalytical Task Group

Consultant on Electrochemistry, U.S. Army, Task Group on Medical Bioengineering R\&D Laboratory

Barbara F. Howell

Alternate Councilor, ACS

Treasurer, ACS of Washington, DC

Member, ASTM, Committee E7 on Nondestructive Testing Subcommittee E7.03 on Liquid Penetrant and Magnetic Particle Task Group E07.03.02 on Magnetic Particle and Penetrant Testing-Penetrant Method

Task Group E07.03.05 on Magnetic Particle and Penetrant Testing

Task Group E07.03.06 on Magnetic Particle Methods

Task Group E07.03.07 on Magnetic Particle and Penetrant Testing-Magnetic Glossary

Willie E. May

Member, ASTM, Committee D19 on Water

President, Washington Chromatography Discussion Group

Dennis J. Reeder

Member, AACC

Study Group on Cortisol

Study Group on Total Serum Protein

Associate Member, IFCC, Expert Panel on Drug Effects in Clinical

Chemistry

Chairman, NCCLS, Subcommittee on Specific Plasma Proteins

Member, NCCLS, Subcommittee on Total Protein 
Robert Schaffer

Member, AACC, Committee on Standards

Subcommittee on Cholesterol

Subcommittee on Creatinine

Subcommittee on Electrolytes in Serum

Subcommittee on Glucose

Subcommittee on Serum Iron

Subcommittee on Theophylline

Subcommittee on Urea

Subcommittee on Uric Acid

Member, NCCLS, Area Committee for Clinical Chemistry

Subcommittee on Calibration Reference Materials

Subcommittee on Quantities and Units

Member, NCCLS, Committee on National Reference System in Clinical

Chemistry, Subcommittee on Nomenclature and Definitions

Rance A. Velapoldi

Working Advisor, ASTM, Committee E7 on Nondestructive Testing,

Subcommittee E7.03 on Liquid Penetrant and MPI Methods, Task

Group E7.03.03 on E-270 Glossary Revision

Member, ASTM, Committee E13 on Molecular Spectroscopy, Subcommittee

E13.06 on Molecular Luminescence

Task Group E13.06.01 on Presentation of Corrected Fluorescence Spectra

Task Group E13.06.02 on Recommended Practices on Molecular Fluorescence Spectroscopy

4. Other

a. Seminars

September 5, 1979 - Ellen Miseo, Polytechnic Institute of New York, "Applications of Correlation Functions to the Study of Relaxation Processes in Liquid Crystalline Systems".

September $6 \quad$ - David Grieble, University of Ohio, "Infrared Spectroscopic Analysis of Hot Gases".

October 5 - George Turrel1, Laval University, "Some Applications of Fourier Transform in Infrared Spectroscopy".

November 8 - Henry Blount, University of Delaware, "Heterogeneous Electron Transfer Kinetics of Biological Molecules".

December 17 - William R. Heineman, University of Cincinnati,

"Optically Transparent Thin-Layer Electrodes:

Studies of Inorganic and Biological Systems". 
January 29, 1980

February 20

February 28

March 6

March 21

Apri1 2

Apri1 16

May 2

May 16

June 6

June 18

July 3

August 4
Otto Grubner, Harvard University, "Improvement in Gas Chromatographic Separation by Specific Activation of Compounds".

- Jimmie Ward, University of Florida, "Some Analytical Applications of Phosphorescence at Room Temperature and Below".

- Jon Maple, University of Tennessee, "Dye Laser Excitation in Matrix Isolation Molecular Fluorescence Spectroscopy".

- Alan Maxwell Bond, Deakin University, "The Use of Microprocessors in Chemical Instrumentation".

- Ed Cehelnik, Governors State University, "Organic Spectroscopy and Photochemistry of DPH".

- Mike Welch, National Bureau of Standards, "Organic Isotope Dilution Mass Spectrometry as a Definitive Method in Clinical Chemistry".

- James Westgard, University of Wisconsin, "Statistical Control in the Chemical Laboratory".

- Alex Fatiadi, National Bureau of Standards, "Chemistry of Oxo- and Pseudo- Oxocarbons".

- Allen J. Bard, University of Texas, "Photoelectrochemistry at Semiconductors".

- Harry S. Hertz and Edward White, National Bureau of Standards, "Mass Spectrometry Competence Building".

- John T. Stock, University of Connecticut, "Historic Instruments: The Scientist's Heritage".

- Electrochemistry Group, National Bureau of Standards, "Review of Progress on the EC Competence Building".

- Petr Zuman, Clarkson College, "Some Applications of Organic Polarography". 
b. SRM Certification

1) SRM 17c, Sucrose

2) SRM 909, Human Serum

3) SRM 910, Sodium Pyruvate (In Progress)

4) SRM 912a, Urea

5) SRM 938, 4-Nitrophenol (In Progress)

6) SRM 1580, Organics in Shale 0 il

7) SRM 1590, Stabilized Wine (In Progress)

8) SRM 1644, Generator Columns for PAH in Water (In Progress) 
IV. Gas and Particulate Science Division

Harry L. Rook, Chief

Ernest E. Hughes, Deputy Chief

\section{A. Division Overview}

The Division is responsible for research on measurement methods for gaseous molecules and for research in the general area of microanalys is. The research is carried out in five groups with scientific effort divided approximately equally between gas measurement research and microanalytical research.

During the past year, substantial progress has been made in the measurement of trace quantities of halocarbons and toxic organic gases. Three new research gas chromatographs (GC) with multiple detectors have been purchased and integrated into the research efforts. The first set of stable gas mixtures containing 0.2 to $20 \mathrm{ppm}$ benzene have been made and a GC procedure has been developed for analys is with a precision of better than one percent. The concentrations of these standard mixtures are currently being verified by comparison to benzene permeation tubes which have been calibrated gravimetrically. This will ultimately lead to a benzene in air Standard Reference Material (SRM) which has its accuracy derived from two independent bases. The procedure developed for benzene will serve as a prototype for the development of other high priority toxic organic gas standards.

The measurement research on $\mathrm{N}_{2} \mathrm{O}$ and halocarbons has been more difficult in that the target concentrations of interest are in the part per billion $\left(10^{-9} \mathrm{~g} / \mathrm{g}\right)$ and in the part per trillion $\left(10^{-12} \mathrm{~g} / \mathrm{g}\right)$ range respectively. Since no absolute preparative or analytical methods exist for these concentration levels, we are developing two independent analytical methods capable of measuring the species of interest. The result of this year's efforts, concentrated on electron capture gas chromatography (EC/GC), are described in detail in the technical accomplishment section. Other plans for next year include the upgrading of the Center infrared (IR) diode laser system and the development of an IR photometric method of analysis for $\mathrm{N}_{2} \mathrm{O}$ as a target species. This work hopefully will offer a new technique for the measurement of diverse gaseous species.

The particulate and microanalysis groups in general have experienced substantial gains in the area of improving existing equipment and obtaining state-of-the-art microprobes. The need to upgrade existing equipment and to obtain new second and third generation equipment has been stressed in recent years. This area is a cornerstone of the Division 5-year plan.

Since last year we have completely refurbished the ion-microprobe, purchased a new $200 \mathrm{KV}$ transmission electron microprobe, and $\mathrm{placed}$ an order for one of the first production models of the new laser microprobe mass analyzer (LAMMA). In addition, a complete laboratory automation facility has been planned and initiated. New microprocessers have been 
purchased for all of the microprobes which require automated data acquisition and processing. An order has been placed for a central controller to tie al1 of the local microprocessers to a central automated system for analytical control and large scale data processing. The complete system, linking the Division's six microprobes into one system should be installed and operating by this time next year. The system will substantially increase analytical speed and versatility on all microprobes. This is very important as the future research emphasis in the microanalytical group will shift to the quantitative analysis of arrays of particles in the two micrometer and smaller size range. Also it is hoped that this automated laboratory system will be a model for other areas in the Center with similar automation needs.

Other areas of progress have been the development of a procedure which allows direct measurement of light absorbing particles in the laser Raman microprobe, the completion of the first asbestos counting grid standard, and the development of a calculation technique to estimate electron beam spreading in thin samples. These research accomplishments will be discussed in detail in the technical accomplishments section of this report.

The future thrust of the Microanalytical Research Group will clearly be in developing analytical procedures for multidimension microanalysis on complex samples or arrays of particles. This will include research conducted under the new competence program in Compositional Mapping.

In summary, the Division has made substantial progress in research related to trace gas measurement and small particle analysis. The personnel level has been stable and significant improvements have been made in equipment necessary to conduct meaningful research at the forefront of analytical science.

\section{Gas Metrology Group: Overview}

The primary responsibility of the Gas Metrology Group is to develop and supply gaseous standards for all areas of science and technology where accuracy in gas measurement is required either by mandate or necessity. To this end, the group has two functions, the development of Standard Reference Materials (SRM's) and the performance of research essential for the characterization of gas standards. In addition a limited gas analysis service, utilizing the unique capabilities of the group, is provided to other groups at NBS and to other government agencies.

Research in the Gas Metrology Group has focused on improving the precision and accuracy of measurement of atmospheric concentration of carbon dioxide, nitrous oxide, halocarbons, and physiologically significant concentrations of several organic species. Development of standards for the accurate measurement of carbon dioxide have progressed to where excellent agreement with accepted international standards has been achieved. As a result a large number of samples are being prepared commercially which will be used to study the feasibility of large scale production of 
highly accurate and stable $\mathrm{CO}_{2}$ gas standards. If production on a large scale is feasible then these standards, as SRM's, will be integrated into the existing measurement network for atmospheric carbon dioxide.

A 1 aboratory has been equipped with state-of-the-art chromatographs for the measurement of trace concentrations of nitrous oxide and halocarbons. Research to improve the precision of measurements has begun and studies are continuing in an effort to assess the accuracy with which these measurements can be made. Concurrently, research is being conducted on new spectroscopic techniques using diode lasers. These techniques, have the potential for sensitivity, reproducibility and specificity equal to or greater than currently employed gas chromatographic methods and, in addition, the nature of the techniques is such that absolute measurements may be possible.

Methods for preparation of accurate and stable standards of benzene and perchloroethylene in air have been studied. These particularly difficult gas systems have required the development of special techniques both for preparation of mixtures of predictable concentrations and for their subsequent analysis.

As in the past the major output of the group has been the analysis and certification of gaseous Standard Reference Materials (SRM's). Over 3400 individual samples of SRM's have been analyzed during the past year. In addition, 85 gaseous SRM's sold by the Office of Standard Reference Materials have been returned by the users for reanalysis as part of a continuing study of the stability of gas standards. It is anticipated that seventeen new SRM's will be issued by the end of FY 1981. These new SRM's include low concentrations of sulfur dioxide in air, nitric oxide in nitrogen, oxygen in nitrogen, and propane in nitrogen, all at concentrations extending the range of existing SRM's.

The joint program between NBS and the Motor Vehicle Manufacturers Association will be successfully completed with the issuance of the last three of twenty-six SRM's intended for measurement of emission from heavy duty motor vehicles.

A document defining the process by which industry-produced gas standards can be related to SRM's has gone through several evolutions of both text and philosophy and is expected to be in a form acceptable to both users and producers within the next few months.

Service analyses performed by the group have included gases obtained from armament compartments of naval aircraft during inflight test firing and analysis of gases generated by 1 ithium batteries under various operating conditions in an attempt to define conditions which result in extremely hazardous situations. In addition, gas standards used by the Mine Safety and Health Administration for quality control of mine atmosphere and the standards used by the State of California as primary reference standards for motor vehicle testing have been analyzed. 
Preliminary work is in progress concerning the accurate measurement of atmospheric oxygen to develop a method sensitive enough to recognize changes in oxysen concentration of one part in 100,000 or less. Modification to a method developed more than a decade ago will be investigated initially with the objectives of improving the accuracy and establishing a new "base line" concentration for oxygen in the atmosphere in 1981 or 1982.

Ernest E. Hughes, Group Leader; William D. Dorko, James W'. Elkins, Joseph A. Mackey, Walter R. Miller, Richard C. Myers, George Rhoderick, William P. Schmidt, Gerald Sleater, James E. Suddueth, William Thorn

\section{Atmospheric Chemistry Group: Overview}

The major thrust of the Atmospheric Chemistry Group has been in the development of measurement techniques for atmospheric species with emphas is on new and improved instrumental procedures. The principal areas of technical expertise are in molecular spectroscopy and radiochemistry. These resources are used in research on determination of fundamental constants required for spectroscopic measurements, molecular cross sections in the ultraviolet, and line intensities and line broadening parameters in the infrared. Research is also conducted on advanced spectroscopic methods for trace analysis, e.g. optoacoustic methods of detection, and in the use of stable and radioisotopes for the characterization of the atmosphere.

Major continuing projects include the following: (a) the measurement of absolute ultraviolet (UV) absorption cross sections for application to the measurement of trace atmospheric gases; (b) development of smallsample radiocarbon measurement techniques for the determination of. sources of individual atmospheric chemical species; (c) development of acoustically-detected Raman gain spectroscopy for the detection of species in the condensed phase; (d) the development of gas phase titration procedures for the intercomparison of measurement standards for $\mathrm{O}_{3}$, $\mathrm{NO}$, and $\mathrm{NO}_{2}$; and (e) the development and evaluation of personal samplers for determining individual exposure to gaseous atmospheric pollutants.

Major new projects initiated during 1980 were: (a) the measurement of absolute infrared band and line intensities for application to the trace measurement of molecules in the gas phase with a tunable infrared diode laser and/or a Fourier transform infrared spectrometer (FTIR); and (b) the development of an improved absolute ultraviolet photometer as a primary standard for the quantitative measurement of ozone.

Some significant accomplishments of the group during 1980 were: (a) the completion of the spectrometer for measuring the temperature dependence of ozone cross sections (at stratospheric temperatures), the determination of the temperature dependence in a critical region (310-320 $\mathrm{nm}$ ) of the Huggins band; (b) an intercomparison among $\mathrm{NO}, \mathrm{O}_{3}$, and $\mathrm{NO}_{2}$ measurement standards by gas phase titration using the simple bimolecular 
reaction, $\mathrm{NO}$ (excess) $+\mathrm{O}_{3}=\mathrm{NO}_{2}+\mathrm{O}_{2}$. Agreement to better than one percent was obtained between the $\mathrm{NO}$ gravimetric cylinder and the $\mathrm{NO}_{2}$ permeation tube Standard Reference Materials and a bias of ca. 4.5 percent was observed between these standards and the current UV photometer measurement standard for $\mathrm{O}_{3}$; (c) an intercomparison of infrared band intensity measurements obtained by the FTIR spectrometer with integrated band intensities obtained from individual line intensity measurements by the tunable diode laser spectrometer; (d) completion of a Raman spectroscopy study on and structure of adsorbed species; and (e) the discovery of a large biogenic (natural) contribution to the volatile organic component of the particulates comprising the Denver brown cloud and a predominately fossil contribution to the elemental carbon portion of the particulate.

Significant research efforts for next year include the following. Measurements of the temperature dependence of $\mathrm{O}_{3}$ cross sections in the most critical region of the Hartley-Huggins bands are planned. Work will be completed and a report prepared describing a personal sampler for nitrogen dioxide, including a wind tunnel evaluation. A major effort will be underway on the development and evaluation of passive personal samplers for toxic organic compounds. A new absolute $\mathrm{O}_{3}$ photometer with improved accuracy and precision will be completed and available for calibration. Ultraviolet cross section measurements will be made for application to the absolute measurement of a series of toxic atmospheric organic compounds. Research will continue in the use of radiocarbon measurements and the new techniques of accelerator mass spectrometry for the characterization of the nature and sources of micro-quantities of atmospheric gases and particulates. Finally a major effort will be initiated on the development of infrared diode laser and FTIR techniques for the absolute measurement of trace gaseous species.

Jimmie A. Hodgeson, Group Leader; Arnold M. Bass, Barry C. Cadoff, Ilan S. Chabay, Lloyd A. Currie, Alan Fried, Robert H. Kagann, George A. Klouda, Juan C. Lerman, James E. Norris

\section{X-ray Fluorescence Group: Overview}

The X-ray Fluorescence Spectrometry Group conducts research in sample preparation including preconcentration methodology applied to a wide range of sample types, develops new techniques for instrument calibration and standardization, and conducts certification analyses on a wide variety of NBS Standard Reference Materials.

The X-ray Fluorescence Spectrometry Group has devoted a major effort in 1980 to the development and characterization of thin films containing known concentrations of metals to serve as calibration standards in the $x$-ray analys is of collected airborne particulate matter. This work, which was part of a joint program between NBS and the United States Environmental Protection Agency (EPA), has recently been published in an NBS/EPA report (EPA-600/7-80-123, June 1980). Our work in extending the sensitivity of the $x$-ray technique to sub-ppm concentration levels through preconcentration as described in NBSIR 80-1995 report is continuing with 
applications to the analysis of SRM biological and botanical samples. The performance of our automated wavelength-dispersive $x$-ray spectrometer which was acquired last year has been evaluated with SRM's with regard to long term stability and measurement precision under computer control and was found to be comparable to the most carefully conducted analyses done manually. In connection with these studies several series of renewal SRM's (alloys and glasses) have been analyzed for homogeneity and elemental composition. Progress has also been made in the development of a comprehensive matrix correction and $x$-ray line overlap computer program for energy-dispersive $x$-ray spectrometric analysis.

In addition to the above programs we $\mathrm{plan}$ to investigate the $\mathrm{x}$-ray absorption spectroscopic technique using synchrotron radiation of the Stanford facility for speciation type studies. This work will be conducted jointly with workers at the Lawrence Berkeley Laboratory.

Peter A. Pella, Group Leader; Edwin C. Kuehner, John S. Sieber

\section{Particle Research Group: Overview}

The objective of the Particle Research Group is to develop quantitative methods for chemical and physical characterization of single particles and of arrays of particles. This includes research into new techniques to interrogate individual particles such as the Particle Doppler Shift Spectrometer and also research into improving current techniques such as our new sulfate aerosol analyser. The group interacts closely with the Microanalytical Research Group but has historically been more concerned with atmospheric particulate research.

The major foci of the Particle Research Group this year have been: (1) completion of the initial stage of the development of the Personal Ambient Aerosol Sampler (PAAS) which includes the design, construction, and characterization of a wind tunnel facility, the characterization of sampler collection efficiency and particle cut-off size, and the evaluation of sampler pumps; (2) completion of the characterization, calibration, and data acquisition-reduction procedures for the Pulsed Electrostatic Precipitator-Flame Photometric Detector (PEP-FPD) Particle Sulfur Analysis System and evaluation of the limiting factors of the system resulting from the response time of the FPD.

The emphas is of our research and development activities in the coming year will be on the use of the wind tunnel facility and aerosol generation techniques for the design and performance evaluation of the PAAS and the preparation of a respirable quartz Standard Reference Material. In both these projects, we will continue to develop techniques for the generation, sampling, dispersion, and size characterization of respirable aerosols and particulates. A smaller effort will also be devoted to the development and characterization of a fast-response FPD to be used in conjunction with the PEP-FPD particle sulfur analysis system. 
Next year the Particle Research Group will be able to take the initiative in responding to both the desire of NBS to provide a respirable quartz SRM and the requests from both the National Institutes for Occupational Safety and Health and ASTM Committee D-22 on Standard Reference Materials for such a material. We will determine the size distribution and distribution stability of candidate materials, coordinate the chemical and phase purity measurements of the samples, and take responsibility for the production of the SRM.

Raymond L. McKenzie, Group Leader; David S. Bright, Robert A. Fletcher

\section{Microanalysis Research Group: Overview}

The Microanalys is Research Group seeks to advance the development and application of instrumental microanalysis techniques for the chemical, crystallographic, and morphological characterization of matter on a micrometer scale of spatial resolution. The techniques utilized include electron probe $x$-ray microanalysis, scanning electron microscopy, transmission electron microscopy, scanning transmission electron microscopy, electron diffraction, energy and wavelength dispersive x-ray spectrometry, secondary ion mass spectrometry, Raman spectroscopy, and laser microprobe mass analysis. Research activities take place on several aspects of each technique, including (1) advancing the understanding of the basic physics of the primary excitation and secondary radiation attenuation;

(2) developing methods of quantitation; (3) developing standards appropriate to each method; and (4) applying the methods to selected problems as a means of assessing limitations in practical analytical situations as well as aiding other NBS research activities.

Highlights of group activities in each area of interest include: (1) X-ray microanalysis - Further development of the NBS characteristic/ continuum method for analysis of particles and rough samples has necessitated determination of an accurate function relating the intensity of the $x-r a y$ continuum to beam energy, continuum energy, and sample composition. This work is nearly complete and should also lead to useful applications in conventional bulk specimen analysis where the background due to the continuum must be accurately determined.

The requirement for accurate peak overlap corrections in quantitative energy-dispersive analys is has led to the development of a calibration method for high resolution wavelength spectrometers so that $x$-ray peak ratios can be directly measured. This spectrometry is combined with an advanced mathematical spectrum fitting technique, the sequential simplex, developed in a joint program with the National Institutes of Health.

A study of the spatial resolution of qualitative and quantitative measurements in analytical electron microscopy has been carried out with Monte Carlo electron trajectory simulation. This study is being expanded to develop deconvolution methods to separate beam spreading effects in thin foil analysis. 
(2) Secondary ion mass spectrometry (SIMS) - The activity in SIMS has been expanded with the hiring of a new SIMS specialist. The ion microprobe mass analyzer is being upgraded to include automatic control of the secondary ion spectrometer. A laboratory mini-computer has been procured for this task and work is currently progressing on interfacing the system to the ion microprobe.

(3) Laser microprobe mass analysis - Procurement of a laser microprobe mass analyzer (LAMMA) has been initiated, with expected delivery in December 1980. Preliminary experiments comparing the laser microprobe mass analyzer with the ion microprobe and ion microscope have been made at manufacturers' 1aboratories and at NBS.

(4) Laser-Raman microprobe - A Raman microprobe with multichannel detection capability has been developed and tested in conjunction with Instruments SA and McClellan Air Force Base (Air Force Technology Assessment Command, AFTAC). This instrument, in operation at McClellan AFB, is being used to obtain Raman spectra from microparticulate samples of specific interest to AFTAC. The multi-channel detection system can significantly shorten the time of data collection, as compared to the single-channel detection system of the NBS instrument, and will prove useful in obtaining Raman spectra from photo-or thermally-sensitive materials.

A major breakthrough has been made in the prevention of the destruction of thermally-sensitive materials when exposed to laser irradiation for Raman analysis. The destruction of the materials made their investigation impossible. The new technique involves encapsulation of the sensitive materials in a non-crystalline (amorphous) layer such as silicate.

The automation of the NBS Raman microprobe has been continued with the procurement of a laboratory mini-computer.

(5) Standards - Substantial progress has been made on the development of analytical electron microscopy standards for asbestos analysis in conjunction with the Environmental Protection Agency (EPA). A correlated counting method involving accurate stage position measurement has been developed to evaluate uncertainties in operator counting data.

Other standards work includes the analysis of the NBS Research Materials Glasses for Microanalysis to upgrade these materials to full Standard Reference Materials (SRM's). In addition extensive work on the characterization of the homogeneity of a stainless steel SRM has been carried out.

In conjunction with other divisions within the Center for Analytical Chemistry, the microanalysis group will initiate a program to develop quantitative compositional mapping techniques on a micrometer scale. This effort will initially involve the electron and ion microprobes, with future extensions to the laser Raman microprobe, analytical electron microscope, and laser microprobe mass analyzer. Crucial to the success 
of this task will be an understanding of the effects of topography and chemical and crystallographic heterogeneity on quantitative compositional analysis. In addition to such fundamental studies, rapid data reduction procedures will be developed so that an entire image can be processed efficiently. The availability of diverse equipment and personnel at NBS provides the basis for the Bureau to become a major center in this rapidly developing area.

In addition to compositional mapping, we are also developing a program involving multi-technique analysis of particles. This program will utilize electron microscopy, secondary ion mass spectrometry, Raman spectroscopy, and laser microprobe mass spectrometry for the ful1 characterization of individual particles. In order to implement the program, it is necessary to develop specimen preparation procedures which will be suitable for a 11 techniques. The combination of instruments available at NBS offers the unique opportunity for this investigation.

Dale E. Newbury, Group Leader; Adville A. Be11, John J. Blaha, Edgar S. Etz, Patrica A. Johnson, Ryna B. Marinenko, Robert L. Myklebust, Patrick J. Sheridan, David S. Simons, John A. Sma11, Eric B. Steel, Barbara B. Thorne 
B. Selected Technical Accomplishments

(Gas and Particulate Science Division)

1. Intercomparison of $\mathrm{O}_{3}, \mathrm{NO}$ and $\mathrm{NO}_{2}$ Measurement Standards by

Gas Phase Titration

Ozone $\left(\mathrm{O}_{3}\right)$, nitric oxide (NO), and nitrogen dioxide $\left(\mathrm{NO}_{2}\right)$ are critical constituents involved in the chemistry and physics of the troposphere and stratosphere and are subject to considerable monitoring requirements. For each of these gaseous constituents, an individual measurement standard is currently employed for calibration. Ozone is measured on an absolute basis by absorption photometry using the known $\mathrm{O}_{3}$ cross section at the mercury $253.7 \mathrm{~nm} 1$ ine. For $\mathrm{NO}$ and $\mathrm{NO}_{2}$ the measurement standards take the form of Standard Reference Materials (SRM's); NO is gravimetrically prepared in cylinders, while the $\mathrm{NO}_{2}$ SRM is a permeation tube. This is the first concerted effort to intercompare gas standards by means of known chemical reactions, in this case employing the rapid, bimolecular reaction,

$$
\mathrm{NO} \text { (excess) }+\mathrm{O}_{3}=\mathrm{NO}_{2}+\mathrm{O}_{2} \text {. }
$$

lt low concentrations of $\mathrm{O}_{3}$ (sub-ppm levels) and in the presence of IXCess NO, the reaction is stoichiometric and the following relationship holds

$$
\Delta(\mathrm{NO})=\Delta\left(\mathrm{O}_{3}\right)=\Delta\left(\mathrm{NO}_{2}\right) .
$$

Therefore a measurement of any one of the three components $(\Delta(N 0)=$ reacted, $\Delta\left(\mathrm{O}_{3}\right)=$ added, $\Delta\left(\mathrm{NO}_{2}\right)=$ produced) yields a measurement of the other two.

In the experimental arrangement, NO from a standard cylinder ( 50 or $100 \mathrm{ppm}$ ) is diluted with clean air by a flow dilution ratio, $\mathrm{R}=30-100$, to obtain an initial NO concentration in the range of $0.5-1.5 \mathrm{ppm}$. The clean air stream passes through a variable $\mathrm{O}_{3}$ source such that $\mathrm{O}_{3}$ can be added in increments over the range, $0-1 \mathrm{ppm}$. The output of the reaction vessel passes to a sampling manifold where the concentrations are monitored with calibrated detectors.

Since the NO sample from the cylinder must be diluted, an accurate determination of the initial NO cylinder concentration requires an accurate determination of the flow dilution ratio, $R$. Considerable effort was expended in verifying the measurement of the NO and clean air flows. A wet test meter calibrated gravimetrically was employed for the air flow and the calibration of this meter was verified at the primary flow calibration facility of the NBS Fluid Engineering Division. The low flow rate $N 0$ measurement was performed with a soap bubble meter, a mercury sealed piston device and a wet test meter, the accuracy of which was confirmed at the primary flow calibration facility. The estimated uncertainty in the measurement of $R$ is less than one percent. 
Our initial objective was to provide an independent analys is of the NO SRM based on photometric $0_{3}$ measurements. Our initial gas phase titration (GPT) measurements, however gave NO cylinder analyses which were consistently 4 - 5 percent lower than the gravimetric analysis. This in turn indicates that the $\Delta\left(\mathrm{O}_{3}\right)$ photometric measurement is less than $\Delta$ (NO) based on gravimetric calibration by the same amount. In an attempt to resolve this bias a specially designed optoacoustic detector was constructed to measure the $\mathrm{NO}_{2}$ produced. This detector uses an argon-ion laser for excitation of the $\mathrm{NO}_{2}$ visible absorption band. The detector is calibrated with an $\mathrm{NO}_{2}$ permeation tube standard and has a minimum detection limit of $.005-.01 \mathrm{ppm}$. An important feature of the optoacoustic detector is that it does not respond to NO. Previous attempts to make similar measurements have used chemiluminescence $\mathrm{NO}_{x}$ detectors $\left(\mathrm{NO}_{x}=\mathrm{NO}+\mathrm{NO}_{2}\right)$ which measure $\mathrm{NO}_{2}$ differentially, i.e. $\mathrm{NO}_{x}-\mathrm{NO}=\mathrm{NO}_{2}$. Considerable measurement uncertainty is introduced when NO is present at higher concentrations, as in the GPT experiment.

A 1 imited number of measurements have been made thus far on $\mathrm{NO}_{2}$ produced, based on a permeation tube calibration, $\Delta\left(\mathrm{NO}_{2}\right)$ pt, but these data are revealing. A considerable amount of data have been obtained comparing $\Delta(\mathrm{NO})$ gravimetric, $\Delta(\mathrm{NO})_{\mathrm{g}}$, and $\Delta\left(\mathrm{O}_{3}\right)$ by photometry, $\Delta\left(\mathrm{O}_{3}\right)_{\mathrm{uv}}$, over the range $0-1 \mathrm{ppm}$. These data were obtained over a one year time scale by two independent operators and comprise 15 different GPT experiments with 44 valid data pairs. These data sets can be fitted by 1 inearregression to yield the following relations:

$$
\begin{aligned}
& \Delta\left(\mathrm{O}_{3}\right)_{u v}=(0.957 \pm 0.002) \Delta(\mathrm{NO})_{g}+(.0002 \pm .001), n=44 \\
& \Delta\left(\mathrm{NO}_{2}\right)_{\mathrm{pt}}=(1.01 \pm 0.01) \Delta(\mathrm{NO})_{\mathrm{g}}-(.0052 \pm .0055), n=8
\end{aligned}
$$

The intercepts are negligible within the uncertainty of the fit. The precision of the GPT measurements is excellent and reflects the precision of the flow measurements as well as the stability of the various detectors. The apparent agreement between $\Delta(\mathrm{NO})$ and $\Delta\left(\mathrm{NO}_{2}\right)$ verifies the reaction stoichiometry and that the GPT apparatus is working properly. We are left with the implication that the $0_{3}$ ultraviolet measurement may be low by approximately 4 percent. This is a larger bias than can be explained by estimates of the uncertainty in the presently accepted value of the $\mathrm{O}_{3}$ cross-section. We are continuing the $\mathrm{NO}_{2}$ measurements for further verification of the above results. In addition a new and improved $\mathrm{O}_{3}$ photometer is being constructed to replace our existing system and an independent $\mathrm{O}_{3}$ calibration will be available soon.

J. A. Hodgeson, A. Fried 


\section{Development of Atmospheric Nitrous Oxide and Halocarbon Standards}

Atmospheric nitrous oxide, and certain halocarbons are considered to be important in that they may have a long-term impact on the concentration of ozone in the stratosphere and consequently the radiation budget of the earth. Measurements at low altitude are used as the basis for predicting mass diffusion into the stratosphere where the critical reactions between ozone and these substances may occur. Large variability of measured concentration (sometimes as high as a factor of 2) have been reported by various laboratories and it is probable that these variations arise from inaccurate standards and from technical problems associated with instruments used for ultra-trace analysis.

Participants at a Workshop on the Measurement of Atmospheric Halocarbons and Nitrous Oxide, held at the National Bureau of Standards, recommended that NBS should prepare a series of Standard Reference Materials (SRM's), at ambient concentration levels for nitrous oxide and some halocarbons.

The suggested gas standards, concentration ranges of the standards in air, and the minimum uncertainties are given in Table 15. The concentration ranges represent the lowest levels at which any gas standards will have been certified as SRM's.

Table 15. Recommended Gas Standards

Substance

1. Halocarbon F-11

2. Ha locarbon F-12

3. Nitrous Oxide

4. Methyl Chloroform

5. Carbon Tetrachloride

6. Methyl Chloride

\section{Concentration Range}

$\begin{array}{ccc}\text { (in air) } & \text { ancertainty } \\ 50-250 \text { ppt } & & 0.7 \% \\ 100-400 \text { ppt } & 0.5 \\ 250-350 \text { ppb } & 0.3 \\ 50-100 \text { ppt } & 2 \\ 50-100 \text { ppt } & 5 \\ 400-800 \text { ppt } & 5\end{array}$

$a_{\mathrm{ppb}}=$ parts-per-billion $\left(10^{9}\right)$

ppt $=$ parts-per-trillion $\left(10^{12}\right)$.

Work on the first three gas standards listed in Table 15 is being funded for a three year period by both the National Aeronautics and Space Administration (NASA) and NBS. The development and research for preparation of the last three gases will begin upon completion of production of the first three standards.

Accordingly, we are investigating the preparation of accurate standards and, as a necessary complementary effort, the improvement of analytical techniques. A laboratory has been completed this year in which state-of-the-art gas chromatographs have been modified to achieve 
the high sensitivity and stability of operation necessary to evaluate the preparation of standards and to accurately assess the concentrations. The approach to the standards preparation involve both the improvement of precision of measurement of these substances and the evaluation of accurate methods of preparation of standards. A preliminary investigation of $\mathrm{N}_{2} \mathrm{O}$ mixtures prepared by weight (gravimetric standards) and mixtures prepared by pressure (manometric standards) indicates a difference between the two methods of no more than 1.5 percent relative at the $300 \mathrm{ppb}$ concentration level. These samples also will serve as references to evaluate the stability of similar mixtures. Preliminary results indicate that no significant changes in the concentration of nitrous oxide in compressed gas mixtures occur over a period of 25 months in aluminum cylinders.

The necessity for extremely sensitive analytical methods has in general resulted in the use of gas chromatography using electron capture detectors for the analysis of atmospheric halocarbons and nitrous oxide. However, the nature of the electron capture detector is such that great skill and considerable experience are required in order to obtain consistent and valid results. Alternate methods of analysis have been surveyed and an investigation was begun of a most promising technique - infrared absorption spectroscopy using a tunable diode laser as a source. This basic method modified by applying second derivative interpretation of the specific lines has yielded most encouraging results in the analysis of nitrous oxide. Figure 27 illustrates the analysis of two samples of nitrous oxide with known concentrations in the ratio of $1.12 / 1.00$. The higher concentration is approximately $300 \mathrm{ppb}$. The sensitivity and reproducibility is comparable to that of electron capture chromatography and it is believed that with modification to the method it could be improved substantially and accordingly a parallel investigation is proceeding comparing the results obtained using the optical technique to those obtained by gas chromatography. 


\section{$259 \mathrm{ppb}$}

\section{$300 \mathrm{ppb}$}
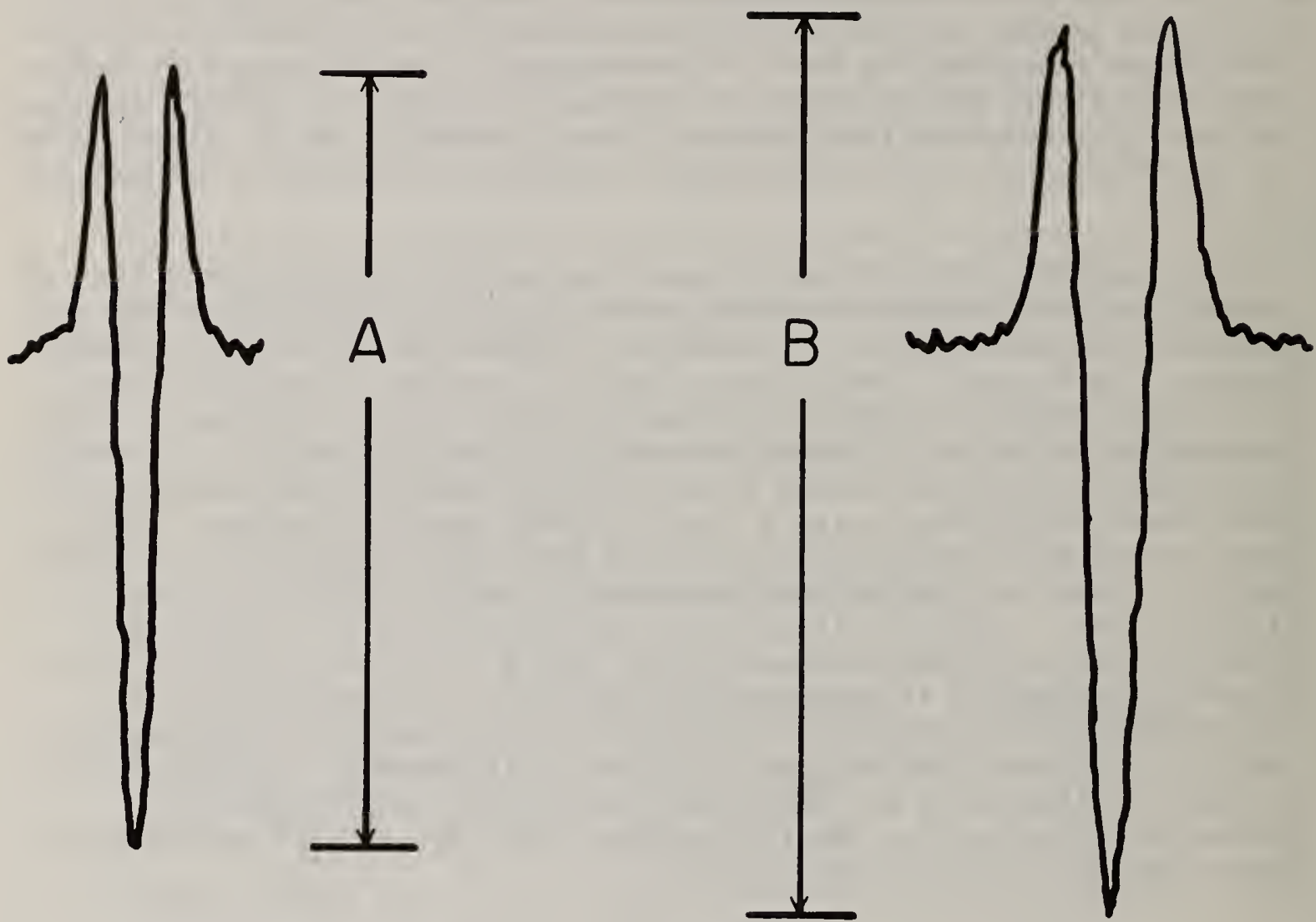

Figure 27. Second derivative signal of the absorption line, $P(26)$, at $2799.73377 \mathrm{~cm}^{-1}$ of the $\nu_{3}$ band of $\mathrm{N}_{2} \mathrm{O}$ for (A) $259 \mathrm{ppb} \mathrm{N}_{2} \mathrm{O}$ and (B) $300 \mathrm{ppb} \mathrm{N} \mathrm{N}_{2} \mathrm{O}$ in air. The ratio of the peak heights $B / A$ is $1.12 \pm 0.02$ ( 4 measurements). The sample pressure is 11 torr with a total optical path length of $15.74 \mathrm{~m}$.

J. W. Elkins, E. E. Hughes 
3. The Determination of Infrared Line Intensities for Selected Molecules Using Diode Laser and Fourier Transform Infrared

\section{Spectroscopy}

There are intensive measurement programs in this and other countries which are a part of a comprehensive effort to investigate the earth's atmosphere. The goal of these programs is to obtain accurate measurements of the concentration profiles of most atmospheric constituents and the temperature profile of the atmosphere. The purpose of these measurements is to predict the response of the ozone layer to changes in concentrations of other constituents, to understand the role of the atmosphere's composition in climate and climate variability, and to monitor pollution. The investigators are increasingly relying on high resolution infrared spectroscopy to make more accurate measurements. But high resolution infrared atmospheric determinations require accurate laboratory measurements of infrared line parameters (i.e. line strengths and line widths as well as wavelengths of line centers). To fulfill these needs, we in collaboration with personnel of the NBS Molecular Spectroscopy Division have initiated a program to measure infrared line strengths and pressure broadening coefficients of atmospheric gases employing both diode laser spectroscopy (DLS) and Fourier transform spectroscopy (FTS). The line parameters also will facilitate our program to apply infrared spectroscopy to the absolute measurement of gas standards.

More than 600 DLS measurements were made on the $v_{1}$ band of $N_{2} 0$. We measured the line strengths of fully resolved absorption 1 ines in the $\nu_{1}$ band of the common isotope $14 \mathrm{~N}_{2} 160$, the associated hot bands and the $v_{1}$ bands of the isotopes, ${ }^{4} \mathrm{~N}_{2} 180$ and ${ }^{14} \mathrm{~N}^{15} \mathrm{~N}^{16} 0$. We also measured the pressure broadening of these lines by the two major atmospheric constituents, $\mathrm{N}_{2}$ and $\mathrm{O}_{2}$, as well as self-broadening. The DLS measurements were made in the 0.5 to 20 torr regime. We estimate the accuracy of our line strength measurements to be within 5 percent and the accuracy of the pressure broadening coefficients to range from 5 to 10 percent.

By least-squares fitting our data to the appropriate band model, band parameters were obtained from which we calculated a band strength for the entire $\nu_{1}$ band system. We also made direct FTS band strength measurements of several $\mathrm{N}_{2} \mathrm{O}$ absorption bands to enable us to cross-check our DLS results.

We can compare the FTS measurements of the band strength of the $\nu_{1}$ band system $\left(1285 \mathrm{~cm}^{-1}\right)$ with the value we determined from our DLS data. We made two separate FTS measurements of this band and obtained the values, $225.3 \mathrm{~cm}^{-2} \mathrm{~atm}^{-1}(295 \mathrm{~K})$, and $227.9 \mathrm{~cm}^{-2} \mathrm{~atm}^{-1}(295 \mathrm{~K})$. These determinations were obtained with 3.62 torr and 6.52 torr $\mathrm{N}_{2} \mathrm{O}$ gas samples, respectively; both samples were pressurized to one atmosphere with nitrogen to ensure adequate pressure broadening. This was required because the effective slit width of the FTS system $\left(0.06 \mathrm{~cm}^{-1}\right)$ was " 100 times larger than the effective slit width of the DLS system. The agreement of the determinations obtained by DLS and FTS is well within 
our estimated uncertainty of the DLS measurement, $220 \pm 11 \mathrm{~cm}^{-2} \mathrm{~atm}^{-1}$. This gives us confidence in the FTS values we obtained for the absolute intensities of other absorption bands in $\mathrm{N}_{2} \mathrm{O}$.

More cross-checks will be performed in the future to enable us to better estimate uncertainties in the FTS determinations. The present calibration is important because we now are measuring absolute intensities in two other atmospheric species, $\mathrm{HNO}_{2}$ and $\mathrm{HNO}_{3}$.

R. H. Kagann

4. The Measurement of Temperature Dependence of Ozone Absorption Cross-Sections in the UTtraviolet

The ozone layer in the stratosphere protects all forms of 1 ife at the surface of the earth from excessive exposure to solar ultraviolet radiation. There is great concern that the emission of substances such as nitrogen oxides and halocarbons into the atmosphere may modify the ozone layer and cause possible harm to life on earth. Measurement of ozone concentrations in the upper atmosphere of the earth is essential in order to detect changes that may be caused by man's activities. Total ozone column density and vertical profiles are determined mainly by means of ultraviolet (UV) absorption spectrophotometry. The interpretation of the atmospheric measurements in terms of accurate ozone concentrations requires a knowledge of the absorption cross-sections of ozone over the range of wavelengths and temperatures involved. Since previously reported cross-section determinations have been limited, both in wavelength and temperature, we have undertaken to make a careful detailed measurement of these absorption cross-sections.

An ultraviolet photometer of novel design was constructed and coupled to a high-resolution scanning monochromator. The photometer is capable of providing absorption measurements of great sensitivity and high accuracy. Radiation from the exit slit of the spectrometer is directed through two sample cells, each 1 meter long, and detected by a pair of photomultiplier tubes. The sample is prepared by ozonizing air or oxygen either by electric discharge or by UV photolys is and then flowing the air and ozone/air streams alternately through the two absorption cells. The changes in light transmittance are detected by the photomultiplier tubes and are recorded in digital form in high speed scalers. The ozone concentration in the sample is determined by measuring the transmittance of the same sample in a short $(1 \mathrm{~cm})$ cell with a fixed wavelength ( $253.7 \mathrm{nnm})$ source and detector system. The cross-section determinations are therefore to be considered as made relative to the accepted value at $253.7 \mathrm{~nm}$ and are not strictly absolute determinations. The sample containers are installed in an adjustable temperature chamber. The apparatus is automated and controlled by a computer so that the spectrum can be recorded in great detail in a minimum amount of time. Our plan is to record the ozone spectrum over the wavelength range 200 to 
$700 \mathrm{~nm}$ and over the range of temperatures 200 to $300 \mathrm{~K}$. Measurements are now underway in the 290 to $350 \mathrm{~nm}$ region.

Figure 28 shows the spectrum recorded for 320 to $310 \mathrm{~nm}$ and at two temperatures $(298$ and $244 \mathrm{~K})$. These spectra were recorded with a spectral resolution of less than $0.02 \mathrm{~nm}$, in steps of $0.05 \mathrm{~nm}$.

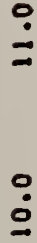

$\stackrel{\leftrightarrow}{-}$

.

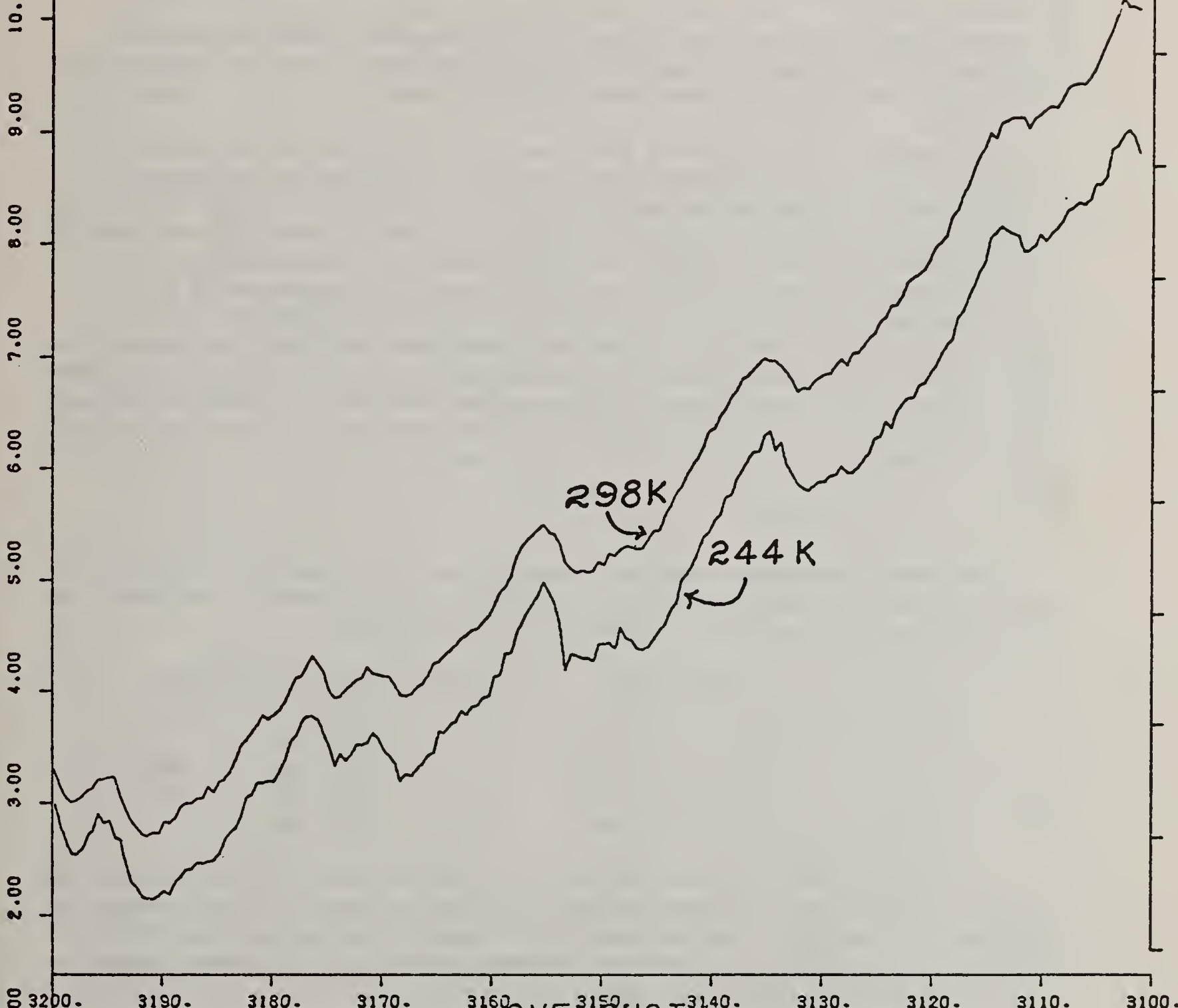

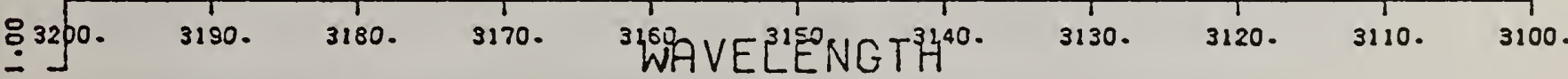

( $\mathrm{nm}$ )

Figure 28. Absorption spectrum of ozone at $298 \mathrm{~K}$ and $244 \mathrm{~K}$. Spectral slit width $0.02 \mathrm{~nm}$; wavelength scanned in steps of $0.05 \mathrm{~nm}$.

A. Bass 


\section{Standards for Measurement of Atmospheric Carbon Dioxide}

Since the early 1950s internationally accepted standards for the measurement of atmospheric carbon dioxide have been traceable to a single set of manometrically analyzed standards prepared by Dr. David Keeling of Scripps Institute of Oceanography (SIO). Expansion of the monitoring network and the necessity for assuring a continuing supply of standards of high accuracy prompted the National Oceanic and Atmospheric Administration and the Environmental Protection Agency to involve the NBS in a program to produce SRM's equal in accuracy to the standards maintained by Keeling. The production of such standards requires a method of preparation of laboratory primary standards that are both accurate and reproducible. An uncertainty of $\pm 0.1 \mathrm{ppm}$ for a standard containing $330 \mathrm{ppm}$ was considered the maximum tolerable error.

A unique microgravimetric technique was developed and a set of 14 standards varying in concentration from 290 to $370 \mathrm{ppm}$ were prepared. The gravimetric technique involves weighing the carbon dioxide contained in a "micro cylinder" on a semi micro balance and accurately transferring it to a large cylinder to which a weighed amount of air is added. The internal consistency of the set was evaluated by intercomparing samples in the set with each other using both gas chromatographic and nondispersive infrared techniques. Painstaking care was taken to assure the highest degree of precision of the measurements. The results showed that the average of the deviations of the samples from the predicted value was $\pm 0.02 \mathrm{ppm}$ and the standard deviation of the observed value relative to the predicted value was $0.09 \mathrm{ppm}$. These standards were then used to analyze three working secondary standard mixtures of $346.7,340.0$ and $333.3 \mathrm{ppm}$, respectively.

The three secondary standards were taken to SIO and were analyzed by that laboratory in common with their own primary standards. A comparison of the NBS and SIO results is shown in the data below:

$\begin{array}{ccc}\text { Sample } & \frac{\text { NBS Value }}{1} & 346.7 \pm .17 \mathrm{ppm} \\ 2 & 340.0 \pm .09 \mathrm{ppm} \\ 3 & 333.3 \pm .10 \mathrm{ppm}\end{array}$

Value Determined at SIO

$346.7 \mathrm{ppm}$
$339.9 \mathrm{ppm}$
$333.2 \mathrm{ppm}$

The limits shown for the NBS value is an estimate of the uncertainty of the concentration of the secondary standard relative to the primary gravimetric standard. The uncertainty of the value measured by SIO is not known on an absolute basis but the repeatability of the measurement is estimated to be about $\pm 0.1 \mathrm{ppm}$.

The agreement is excellent considering the complete independence of approach of each laboratory. However, further work is p? anned to improve the precision of the measurements and thereby to determine whether the slight differences are random errors or biases between the methods. The 
most probable source of error in the NBS value would be in the analysis of the reagent gases while the most likely source of error at SIO would be associated with volume calibrations. Both of these error sources are being investigated.

E. Hughes, G. Sleater

\section{Industrial Preparation of Traceable Gas Certified Reference Materials}

The demand for gas standards is increasing at an accelerated rate. Improvement in instrumentation during the last two decades and mandated requirements for accuracy in gas measurements related to environmental concern have both contributed to the demand. NBS 1 imitations imposed on the gaseous SRM program resulted in severe shortages of standards which on occasion has caused concern among regulatory agencies, users and producers. Since it is not realistic, either philosophically or physically, to consider expanding the gaseous SRM program beyond its present bounds, it has become essential to establish some alternative supply of accurate standards. As a result of this need a protocol has been developed which in essence transfers the responsibility for producing large numbers of accurate standards (Certified Reference Materials) to the specialty gas industry. The protocol makes provision for monitoring by the regulatory agencies and at the same time NBS retains the responsibility for assuring traceability of the Certified Reference Materials (CRM's) to NBS gaseous SRM's.

According to the protocol, the specialty gas producer will prepare CRM's at concentrations equal to or very close to the concentration of existing SRM's. These will be prepared in large homogeneous batches. The producer will analyze each CRM from the batch by comparison to an SRM. The regulatory agency will select samples from each batch for analysis. The analysis by the regulatory agency will be performed using SRM's as calibrants. Both the producer and the regulatory agency will furnish NBS with complete information concerning the production and analysis of the CRM's including the data for both calibration of the instruments used and the analysis of the CRM's. NBS will then statistically compare the results reported by the producer with those found by analysis by the regulatory agency. In addition, NBS will examine all the results reported by the producer to ascertain the homogeneity of the batch. From these data it will be possible to verify the total uncertainty of the concentration claimed by the producer. At this point the concerned parties will be notified that the particular batch of CRM's has been prepared in accordance with the protocol and that the stated concentration is correct within the defined limits of uncertainty.

The protocol in its present form has been extensively modified in response to comments from the gas industry, users of SRM's and the principal regulatory agency, the Environmental Protection Agency. It is not a "cook book" by which CRM's can be prepared. Rather, it recognizes the experience and expertise of the specialty gas industry while at the 
same time it recognizes the reservations which the user may have regarding the industry and which the industry may have regarding the regulatory agency. It is expected that the role of NBS as an independent overseer will inspire confidence not only in the quality of the CRM's but in the integrity of the entire procedure.

The availability of CRM's should moderate the demand for the corresponding SRM's, so that the latter would be used largely as primary standards, which was the purpose for which they were originally produced.

E. E. Hughes

7. Standards for Measurement of Benzene and Perchlorethylene in Workplace Atmospheres

The increasing concern with the potential hazards associated with certain organic compounds in industrial workplace atmospheres has emphasized the need to solve the difficult problem of standardization of the measurement of low concentrations of intermediate molecular weight volatile organic compounds. The compounds of interest include benzene, toluene, perchlorethylene, chloroform, carbon tetrachloride, ethylene dichloride, and tetrachloroethylene. Work completed several years ago at NBS indicated that mixtures of these compounds in air at high pressure in steel cylinders could not be prepared with predictable stability. However, aluminum cylinders, which have reduced adsorptive and absorptive properties of the inner surface compared to steel cylinders, appeared to be capable of containing organic mixtures with reduced losses.

Consequently, mixtures of benzene in air and perchlorethylene in air were prepared by a micro-gravimetric technique developed at NBS and the concentrations were determined over a period of several months. The benzene mixtures which ranged in concentration from 0.2 to $10 \mathrm{ppm}$ were found to have the same relative concentration, within the uncertainty of the analytical technique, over this period. The usual difficulties associated with the analysis of sub-ppm concentrations of benzene in air samples were encountered during these determinations. The effects of the non-linearity of response to concentration ratios, the imprecision of integration for small peaks and the drift of the flame ionization detector response due to slow changes in instrument and sampling conditions were moderated by using multiple-standard calibration curves and careful sampling techniques. The elimination of the interference caused by a cylinder contaminant (probably residual trichloroethylene) was accomplished by the careful selection of the column parameter (i.e., length, material, temperature), and carrier-gas flow rates.

The gravimetric method used to prepare the air mixtures of benzene or perchlorethylene was devised to overcome the problem of transferring a vapor at low pressure into a cylinder without contamination by atmospheric air. The organic liquid was introduced into a previously weighed 
capillary tube which was then sealed and reweighed to determine the weight of the.organic. The capillary tube was then placed in a short length of flexible tubing which in turn was connected to the weighed sample cylinder. The capillary was broken inside the flexible tube and the contents were flushed totally into the sample cylinder. The sample cylinder was then filled with the diluent gas whose mass was determined by difference after weighing the filled sample cylinder. Gravimetric standards with uncertainties in their concentrations of less than \pm 1 percent relative can be thus prepared.

Alternatively standards may be generated by permeation tubes containing the substance of interest. However, the accuracy of mixtures generated with permeation tubes depend to a great extent on the stability of the permeation rate. Tubes containing a number of different organic compounds were studied over a period exceeding one year. The results showed that tubes containing benzene required as much as one month to reach equilibrium at constant temperature. Tubes containing other organic compounds required greater or lesser times depending on the particular organic involved. The rates after equilibrium, however, were found to have uncertainties of less than \pm 1 percent relative.

It appears that permeation tubes may serve as adequate standards providing the conditions under which they are kept are carefully controlled and if frequent measurements of the permeation rate are made.

W. P. Schmidt

8. Motor Vehicle Manufacturers Association Program for Heavy Duty Vehicle Emissions Standards

In 1978 a joint program was initiated between NBS and the Motor Vehicle Manufacturers As sociation (MVMA). The goal of the program was to produce twenty-six new gaseous SRM's which are required by the motor vehicle industry for emission testing of heavy duty vehicles to insure compliance with government regulations. The production of these new SRM's would have been impossible within existing NBS programs because of constraints imposed by the already heavy commitments to produce other gaseous SRM's.

This program ended successfully in September of 1980. Twenty three new SRM's have already been issued by the Office of Standard Reference Materials and the final three will be available in the near future.

The program has benefited NBS in a number of ways. For instance, the development of ten new carbon monoxide in nitrogen mixtures involved measurements which demonstrated the usefulness of high-pressure injection from large sample loops into a gas chromatograph to increase ultrasonic detector response for carbon monoxide mixtures at concentrations of less than $100 \mathrm{ppm}$. The technique allowed the intercomparison of samples with 
standards with a precision of \pm 0.2 percent. Certification of these SRM's involved the first test of the software used to control the combined gas chromatography NBS-developed computer operated-gas analys is system (COGAS). COGAS had previously been used only with continuous flow gas analyzers.

The $100 \mathrm{ppm}$ propane mixtures containing 0 percent, 5 percent and 10 percent oxygen, when used with an existing SRM containing 20.95 percent oxygen, permits the study of flame ionization detector (FID) response characteristics as a function of oxygen content. This is important to allow the optimization of air to fuel ratios for individual FIDs used in direct combustion exhaust measurements.

The final three SRM's to be issued under this program are 5, 10, and $20 \mathrm{ppm}$ nitric oxide in nitrogen. Nitric oxide is not particularly reactive except toward oxygen with which it readily combines. The resulting nitrogen dioxide is quite reactive and may be lost from the gas phase of a cylinder by reaction on the cylinder walls. Careful cylinder treatment and the elimination of all oxygen from the system is essential for the preparation of stable mixtures of nitric oxide. A number of mixtures of low concentrations of nitric oxide in nitrogen were prepared manometrically in aluminum cylinders. Analyses were performed over a period of 18 months and no evidence of instability of the mixtures was found. Consequently, it was possible to prepare similar mixtures by the more accurate gravimetric techniques. These mixtures were then used as laboratory standards for the analysis of the SRM's.

The potential SRM's were purchased commercially in lots of 50 at each concentration. Unlike most other SRM's, the lots were not entirely homogenous and in addition some evidence of a very slow decrease in concentration was observed for some samples in each lot. For these low concentrations of nitric oxide each sample must be individually certified.

A11 mixtures of nitric oxide in nitrogen contain small quantities of nitrogen dioxide. The quantity may be relatively small in mixtures containing large amounts of nitric oxide but these quantities of nitrogen dioxide may constitute a significant fraction of the total oxides of nitrogen $\left(\mathrm{NO}_{x}\right)$ in low concentration mixtures. Chemiluminescent analysis may be used to differentiate the two species but the uncertainty is great. A chemical method, the Saltzman Method, may also be used. However, this method is quantitated by using mixtures of nitrites at various concentrations. A factor of about 0.72 is usually applied to the results to compensate for the "non-stoichiometric" reaction. A modification to this method has been developed which involves the use of high nitrate concentrations in the absorbing solution which not only increases the factor to 1.0 but eliminates a previously observed concentration dependence of the factor. This method, using nitrite standards, in a high concentration nitrate solution, has been used to measure concentrations of nitrogen dioxide generated using permeation tubes and the calculated and predicted values agreed within 1 percent relative. 
The NBS/MVMA joint program is a good example of the type of positive, synergistic venture between industry and NBS envisioned in the establishment of the NBS Research Associate Program, leading to important accomplishments beneficial to all involved. The legacy of improved measurement techniques and the more than 100 new gravimetric standards resulting from the program will benefit NBS' ongoing programs; while the availability of these desperately needed SRM's, not otherwise possible without the program, will allow clean air milestones to be met by the motor vehicle industry.

W. Thorn, R. Miller, E. E. Hughes

\section{Evaluation of the Coherent Anti-Stokes Raman Spectroscopy (CARS) Capillary Waveguide Detection System for High Performance Liquid Chromatography}

In previous characterizations of organic materials conducted with coherent anti-Stokes Raman spectroscopy (CARS), a capillary waveguide sample cell was found to increase the detectability of the CARS signal by two orders of magnitude when compared to the $1 \mathrm{~cm}$ path length cuvette cel1 normally used [1]. The capillary waveguide is most efficient when it incorporates a very small internal diameter and a very long path length and can be used to lower the detection limits for non-resonant CARS to approximately $10^{-4} \mathrm{M}$. Resonant-enhanced CARS (pumping 1 aser frequency approaches an allowed electric dipole transition frequency) shows a lowering of the detection limits to $10^{-6} \mathrm{M}$. A combination of resonant-enhanced CARS and the capillary waveguide sample cell would be expected to achieve detection 1 imits to $10^{-8} \mathrm{M}$.

Perylene (a polycyclic aromatic hydrocarbon) could easily be detected at concentrations of $10^{-4} \mathrm{M}$ in toluene under non-resonant conditions in the capillary waveguide. An example is presented in figure 29. The bands of perylene are indicated by a $P$. No spectral features could be observed when resonant-enhanced conditions were used. This is due to the fact that all of the CARS signal is absorbed by the sample due to the extremely strong absorbance at the wavelength of resonant-enhancement. Thus, resonant-enhancement of the CARS signal is not possible with a capillary waveguide. These results have given insight into the capabilities and limitations of the capillary waveguide and have formed a basis for further examinations of detection schemes using CARS. 


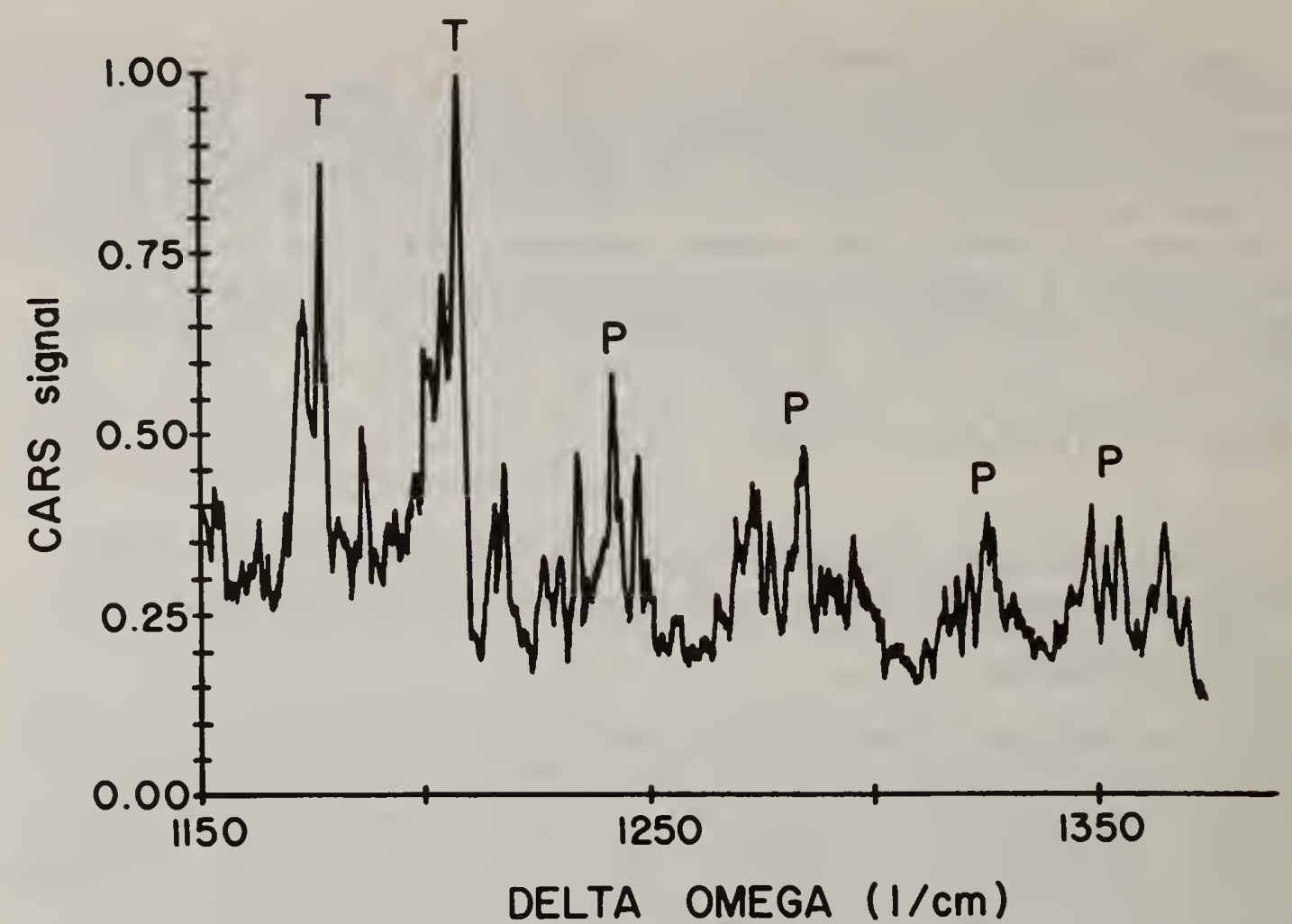

Figure 29. Coherent anti-Stokes Raman spectrum of $2 \times 10^{-4} \mathrm{M}$ perylene in toluene taken under non-resonant conditions. Toluene bends are marked "T" and perylene bands are marked "P". Pumping laser wavelength is $540.00 \mathrm{~nm}$. Scanning laser wavelength varies over the range 575.75 to $583.31 \mathrm{~nm}$ in $0.02 \mathrm{~nm}$ increments. Thirty-six pulses per point data collection. Full scale is $0.02456 \mathrm{~V}$ analog signal normalized to 1.00 .

In order to utilize resonant-enhanced CARS to obtain detection limits at the $10^{-7} \mathrm{M} \mathrm{level,} \mathrm{a} \mathrm{single} \mathrm{pass} \mathrm{cell} \mathrm{with} \mathrm{a} \mathrm{very} \mathrm{short} \mathrm{path-}$ length appears to be necessary. A cell of this type has been developed by Carreira, et al [2]. Detection limits for many organic materials have been reported at $10^{-6}$ to $10^{-7} \mathrm{M}$ levels. This type of detection system, when coupled with such instrumentation as high performance liquid chromatography (HPLC), gives detection limits that are much lower than have previously been realized by CARS and also allows molecular specificity which is not present in other detectors currently used for HPLC.

\section{References}

[1] Schaefer, J. C., and Chabay, I., Generation of Enhanced Coherent Anti-Stokes Raman Spectroscopy Signals in Liquid-Filled Waveguides, Optics Lett. 4, 227-229 (1979). 
[2] Carreira, L. A., and Rogers, L. B., New Liquid Chromatographic Detection System for Environmental Pollutants, EPA Research Report EPA-600/4-80-015, February 1980.

J. J. B1aha, J. C. Schaefer

10. Measurement of Ambient Sulfur Aerosols By a New Fast Response Flame Photometric Instrument

The development and characterization of the Pulsed Electrostatic Precipitator-Flame Photometric Detector (PEP-FPD) system for the measurement of ambient level aerosol sulfur concentrations has been completed this year and a final report to EPA is in the review process. The basis of the technique is the use of the PEP to selectively modulate the a erosol component of the sample while leaving the gas phase component unaffected, resulting in a signal from the detector (FPD) which has two separable components corresponding to the particle and gas phase sulfur concentrations. Because the FPD response to sulfur concentration is non-linear (Response $\sim[S]^{n}$ where $n=1.5$ to 2 depending on various flame and flow parameters) the sensitivity of the FPD is a function of the total sulfur concentration in the sample. The following data shows the sensitivity (dI/dS) and sensitivity relative to sensitivity at $1 \mathrm{ppb}$ for our FPD.

\begin{tabular}{cccc} 
[S] $(\mathrm{ppb})$ & & $\mathrm{dI} / \mathrm{dS}(\mathrm{amp} / \mathrm{ppb})$ & $\mathrm{dI} / \mathrm{dS}$ relative to $1 \mathrm{ppb}$ \\
\cline { 1 - 1 } 1 & & $1.42 \times 10^{-10}$ & $(1)$ \\
5 & & $4.81 \times 10^{-10}$ & 3.4 \\
10 & & $8.13 \times 10^{-10}$ & 5.7 \\
50 & $2.75 \times 10^{-9}$ & 19.4 \\
100 & $4.65 \times 10^{-9}$ & 32.7 \\
200 & $7.86 \times 10^{-9}$ & 55.4 \\
500 & $1.57 \times 10^{-8}$ & 110.8 \\
1000 & $2.66 \times 10^{-8}$ & 187.3
\end{tabular}

One consequence of this operational characteristic is that it is possible to increase the sensitivity of the PEP-FPD system by increasing the total sulfur concentration in the flame. We have verified this by adding a constant concentration of $\mathrm{CH}_{3} \mathrm{CH}_{2} \mathrm{SH}$ to the hydrogen. A second consequence, however is a complication of data reduction when one is attempting to measure more than one sample component because the sensitivities are related to total sulfur concentration, not to sample component concentrations. We have solved this problem by developing a data acquisition/ reduction procedure that enables us to achieve simultaneous measurement of aerosol and gas phase sulfur concentrations with sensitivities of 0.2 $\mu \mathrm{gm} \mathrm{S} / \mathrm{m}^{3}$ and $1 \mathrm{ppb}$ respectively. Initial data comparison from an intercomparison study shows a very high correlation coefficient with data obtained by a dichotomous sampler-x-ray-fluorescence system. 
We have shown the major limitation in response time of the technique is in the FPD. (The commercially available FPDs have $>60 \mathrm{sec}$ rise times and $\sim 1.5 \mathrm{sec}$ fall times.) We have completed characterization of a new FPD design which has symmetric rise and fall times of less than one second. This faster response time means the PEP-FPD system will be capable of real-time measurements of ambient $\mathrm{SO}_{2} / \mathrm{SO}_{4}$ concentrations under conditions where the concentrations are dynamic, such as in plume traverses. Additional research will be needed to improve the sensitivity and flow stability of the fast-flame FPD to its full potential, though it now is comparable to commercially available FPDs in those respects.

R. L. McKenzie, D. S. Bright, R. A. Fletcher

11. Monte Carlo Electron Trajectory Calculation of Beam Spreading Effects in Thin Foils in the Analytical Electron Microscope

Analytical electron microscopy (AEM) is a rapidly developing microanalytical technique with enormous potential for application throughout the physical and biological sciences, as well as in many other problems of technological importance. The AEM technique combines the transmission electron microscope (TEM) with the scanning transmission electron microscope (STEM), resulting in a composite instrument which has the capability of forming probes on the nanometer scale. Both energy dispersive x-ray spectrometry (EDS) and electron energy loss spectrometry (EELS) are used for analysis. A major question which is under current study at a number of laboratories concerns the role of electron scattering in limiting the spatial resolution of the technique, particularly at interfaces.

To investigate scattering in thin foils containing small structures of different composition from the matrix, we have adapted the Monte Carlo electron trajectory simulation technique previously developed in our group for bulk specimen analys is to the thin foil case [1]. Calculated relative $x$-ray intensity profiles across interfaces in the $\mathrm{Fe} 0-M g 0$ system were found to be in good agreement with experiments published in the 1 iterature $[2,3]$. These calculations were then extended to examine the effects of beam spreading on quantitation methods for AEM analysis. Quantitative analysis is usually carried out by means of a sensitivity factor approach as suggested by $\mathrm{Cl}$ iff and Lorimer [4]. This method presupposes that the region under electron bombardment is homogeneous, a condition which is not achieved when beam spreading occurs at interfaces. Monte Carlo calculations of the apparent concentration of iron in a pure FeO slab at an MgO grain boundary as a function of slab width ( $w$ ) and foil thickness ( $t$ ) are shown in figure 30. Only when the slab width is large compared to the incident beam size does the calculated iron composition approach the true value (dotted line). The value of the broadening, $b$, predicted by a simple model is shown for comparison [5]. Further calculations and experiments will be made to develop deconvolution techniques suitable for deriving accurate compositional values when fine structures are analyzed in the AEM. 


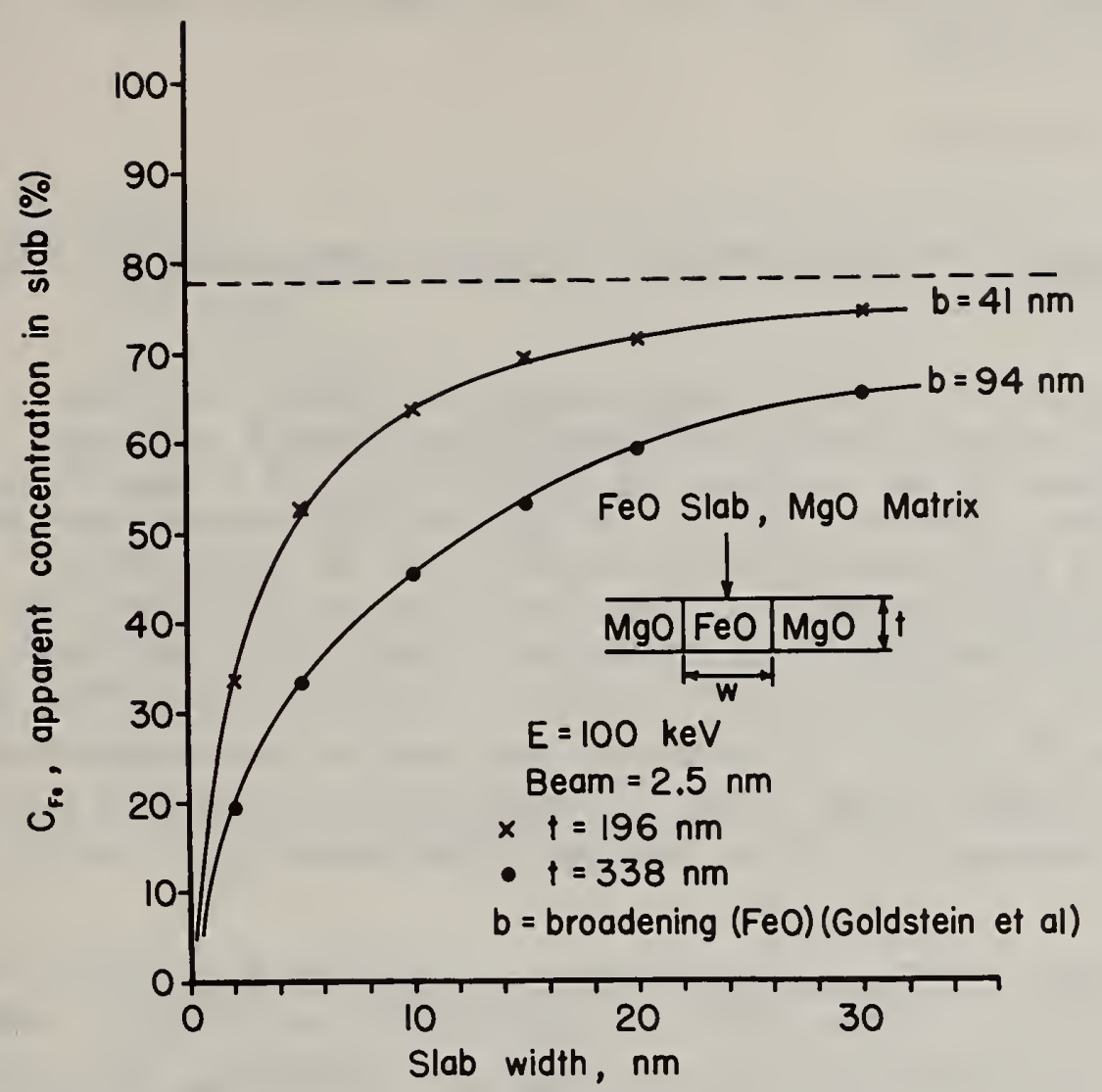

Figure 30. Calculation of the apparent iron concentration measured in a pure $\mathrm{Fe} 0$ slab at an MgO grain boundary.

References

[1] Myklebust, R. L., Newbury, D. E., and Yakowitz, H., NBS Monte Carlo Electron Trajectory Calculation Program, in NBS SP460, Use of Monte Carlo Calculations in Electron Microscopy, 105128 (1975).

[2] HalT, E. L., and Vander Sande, J. B., The Effect of Sample Thickness on High Spatial Resolution Composition Profiles in Dedicated STEM, in Proc. 37th Ann. Conf. Electron Microscopy Soc. America, 474-475 (1979).

[3] Newbury, D. E., and MykTebust, R. L., Calculation of Electron Beam Spreading in Composite Thin Foil Targets, in Microbeam Analys is-1980, ed., D. Wittry, San Francisco Press, 173-175 (1980).

[4] Cliff, G., and Lorimer, G. W., The Quantitative Analys is of Thin Specimens, J. Microscopy 103, 230-207 (1975). 
[5] Goldstein, J. I., Costly, J. L., Lorimer, G. W., and Reed, S. J. B., Quantitative $X$-ray Analysis in the Electron Microscope, in SEM/ $1977,315-324$.

D. Newbury, R. Myklebust

12. General Expression Developed for the X-Ray Bremsstrahiung Intensity $\underline{\text { Data }}$

The quantitative electron probe analysis of samples with the various methods based on peak-to-background measurements requires detailed knowledge of the continuum (bremsstrahlung) as a function of specimen and beam parameters [1-3]. During the developmental work on FRAME $P$, the NBS peak-to-background procedure for the analys is of particles and rough surfaces, we attempted to use various functions from the 1 iterature to describe the observed variations in the continuum intensities, with respect to atomic number $Z$, accelerating voltage $E_{0}$, and $x$-ray energy $E_{V}[4-6]$. Since these functions did not adequately describe the observed values of the continuum intensity we have been developing an expression relating the continuum intensity, $I$, to $Z, E_{0}$ and $E_{V}$.

In this study, $1000 \mathrm{sec}$ live-time spectra have been run for 44 pure element targets at $E_{0}$ values of $10,15,20,25,30$, and $40 \mathrm{keV}$. Intensity values for several different energy windows were determined for each spectrum. The midpoint energy of each window was used as the value for $E_{v}$. The measured $x-r a y$ intensities, were converted to generated intensities by correcting for target absorption, electron backscatter loss, detector absorption, and incomplete charge collection.

A computer was used to fit equations for $I$ vs. $Z$ at the various $E_{0}$ values. It was found that all the curves fit the general equation $I=a Z^{n}$. Figures 31 and 32 are plots of $[-\ln (a)]$ and $n$ as a function of $\left(E_{0} / E_{\nu}\right)-1$ where $E_{0} / E_{V}$ is equal to the overvoltage, $U$. Two important aspects of a and $n$ which are shown by the plots are: First both plots follow the same general family of curves, hence the overvoltage dependence is the same or similar for both terms; second, the plots show that the two terms have a minimum at a U-T value of about 10 . We are currently working on development of expressions to define the dependence of a and $n$ on overvoltage.

\section{References}

[1] Smal1, J. A., Heinrich, K. F. J., Fiori, C. E., Myklebust, R. L., Newbury, D. E., and Dilmore, M. F., The production and characterization of glass fibers and spheres for microanalysis, SEM/1978/I., 445-454. 
$-\operatorname{Ln} a$ in equation $I=a Z^{n}$ vs. $U-1$

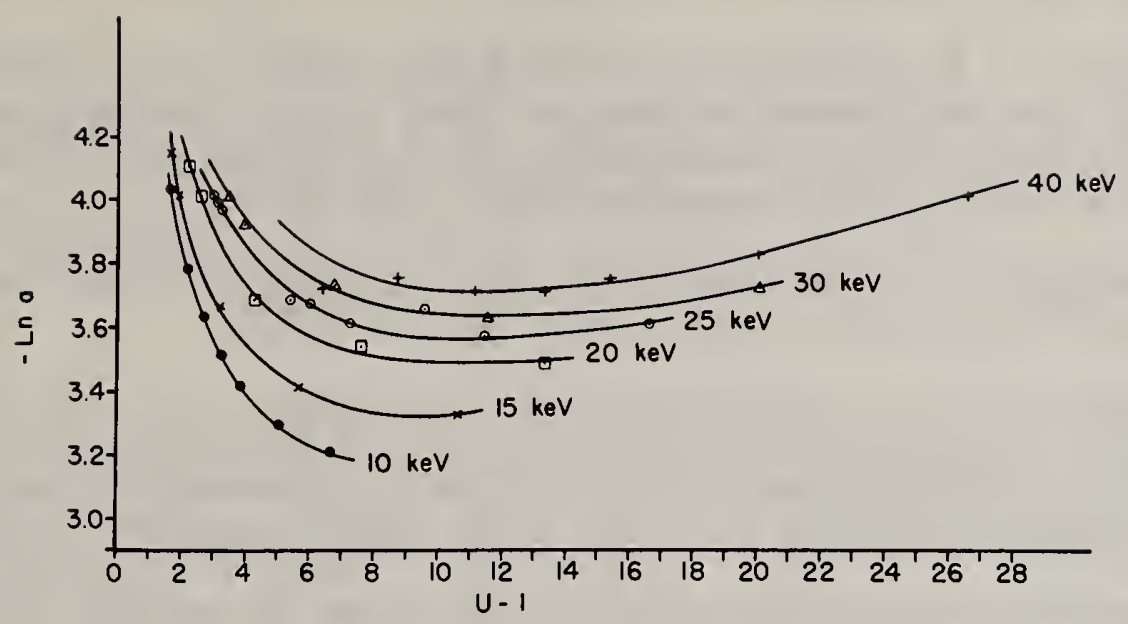

Figure 31. Plot of $-1 n$ a vs. U-1 for different values of $E_{0}$.

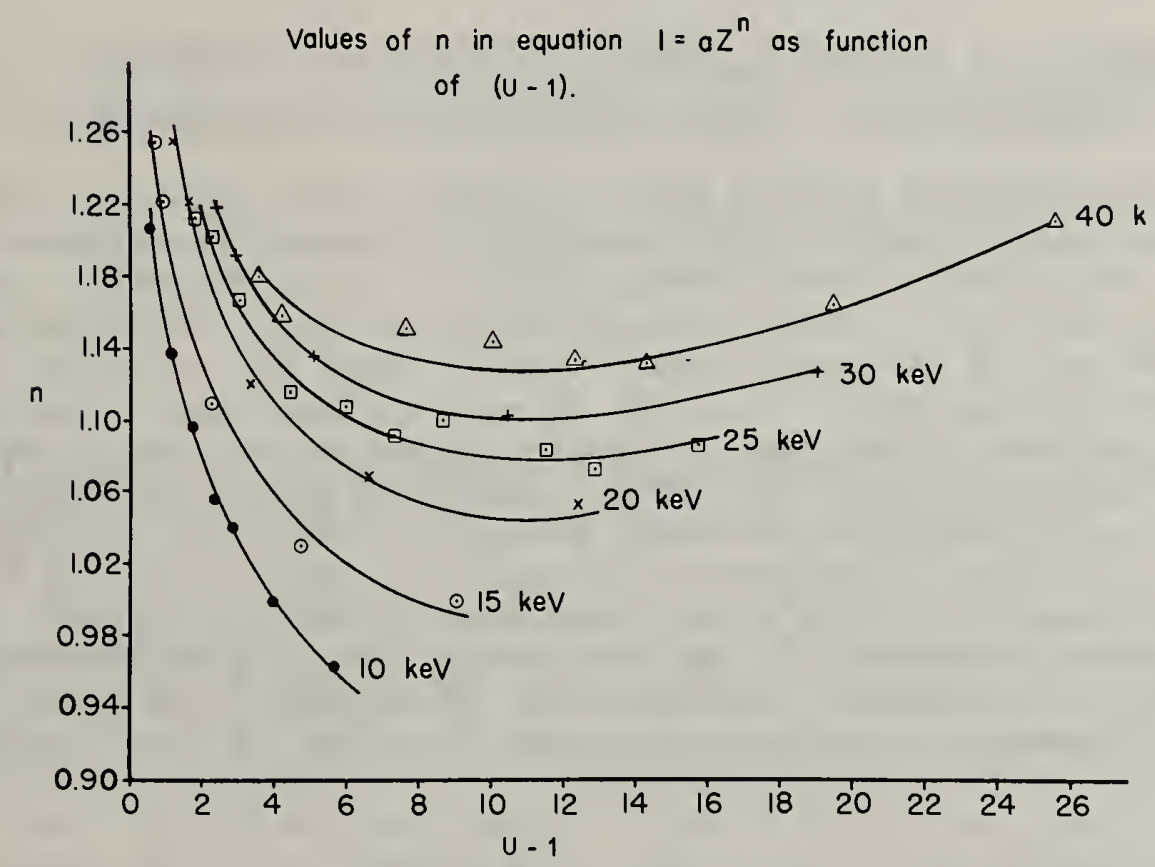

Figure 32. Plot of $n$ vs. U-T for different values of $E_{0}$. 
[2] Statham, P., and Pawley, J., A new method for particle x-ray microanalysis based on peak-to-background measurement, ibid., 469-478.

[3] Sma 11, J.A., Newbury, D. E., and Myklebust, R. L., Analys is of particles and rough surfaces by FRAME $P$, a ZAF method incorporating peak-to-background measurements, Microbeam Analysis--1979, San Francisco, San Francisco Press, 243 (1979).

[4] Kramers, H. A., On the theory of $x$-ray adsorption and the continuous $x$-ray spectrum, Phil. Mag. 48, 836 (1923).

[5] Smith, D. G. W., Gold, C. M., and Tomlinson, D. A., The atomic number dependence of the $x$-ray continuum intensity and the practical calculation of the background in energy dispersive electron microprobe analysis, X-ray Spectroscopy 4 , 149 (1975).

[6] Statham, P. J., Generation, absorption and anisotropy of thicktarget bremsstrahlung and implications for quantitative energydispersive analysis, $\underline{X-r a y}$ Spectroscopy 5, 154 (1976).

J. A. Sma11, D. E. Newbury, R. L. Myklebust

\section{Development of a Sequential Simplex Procedure for Resolving} Spectral Interference in Energy Dispersive X-ray Spectrometry

The lithium-drifted silicon [Si(Li)] detector has become an established tool in $x$-ray spectrochemical techniques such as $x$-ray fluorescence spectrometry and electron probe microanalysis. The instrumental response of the $\mathrm{Si}(\mathrm{Li})$ detector is closely approximated by a Gaussian distribution having a width at $1 / 2$ the peak height which is about 100 times the natural peak width of an $x$-ray line. In order to resolve individual $x$-ray peaks in an $x$-ray spectrum containing overlapping peaks, we have developed a sequential simplex procedure for optimal selection of the parameters in a mathematical expression that describes a spectral peak [1]. In this procedure, each of the $n$ independent variables in the function to be fitted is assigned an axis in an n-dimensional coordinate system. A simplex, in this coordinate system, is defined to be a geometric figure consisting of $n+1$ vectors. The simplex is moved toward the set of independent variables that optimize the fit according to a set of specific rutes.

The energy-dispersive $x$-ray peaks were assumed to be Gaussian line profiles; therefore, the function to be fitted is:

$$
Y_{j}=\sum_{i=1}^{n} A_{i} \exp \left[-1 / 2\left(\frac{E_{j}-E_{i}}{\sigma_{i}}\right) 2\right]
$$


where $Y_{j}$ is the intensity at energy $E_{j}, n$ is the number of $x$-ray peaks and $A_{j}, E_{j}$, and $\sigma_{i}$ are respectively the amplitude, energy and width of peak $i$. For each peak, the parameters to be determined are $A_{i}, E_{i}$, and $\sigma_{i}$. The total number of independent variables $(n)$ is therefore $3 n$. We have reduced the number of variables by: (1) determining the energies of all minor peaks from the energy of the principal peak; and (2) determining the widths of all minor peaks from the width of the principal peak. This reduces the number of independent variables to $n+2$ thereby improving the chance of obtaining a good fit with a minimum of computer time [2].

This procedure has been used to determine the $K_{\beta} / \Sigma K$ line ratios for elements of atomic number 15-32 by energy-dispersive anaiysis [3].

The method allows us to separate $x$-ray lines that are separated by only $60 \mathrm{eV}$ when the resolution of the detector is $160 \mathrm{eV}$. The procedure was extensively tested this year and is now being extended to the determination of the $L$ line ratios.

\section{References}

[1] Spendley, W., Hext, G. R., and Hinsworth, J. R., Technometrics $\underline{4}, 441$ (1962).

[2] Fiori, C. E., and Myklebust, R. L., 1978 DOE Symposium Seris CONF-780421, p. 139.

[3] Heinrich, K. F. J., Fiori, C. E., and Myklebust, R. L., J. Appl. Phys. 50 (9), 5589 (1979).

R. L. Myklebust

14. Development of a New Procedure for Calibration of a Crystal Spectrometer for Relative Line Intensity Measurements

In recent years quantitative electron probe micro-analysis (EPMA) with energy-dispersive $x$-ray spectrometry (EDS) has been proved to be as valid as wavelength dispersive (WDS) analysis in most cases. The major limitation though of EDS is its poor resolution, which necessitates data reduction procedures to make peak-overlap corrections. Such corrections require accurate knowledge of relative $x$-ray line intensities.

For the elements phosphorus to germanium, $\mathrm{K}-\mathrm{l}$ ine ratios have been determined by several authors, including a group from this laboratory [1]. Accurate determinations of the K-lines have been made possible by EDS where detector efficiency corrections can be calculated for contribution from the beryllium window, gold contact layer, silicon dead layer, and detector acceptance angle. Overlaps between lower atomic 
number $K_{\alpha}$ and $K_{\beta}$ peaks can be easily handled by a modification of a simplex procedure [2].

Because the overlap of L-lines in EDS is far more extensive EDS cannot be used for relative line intensity measurements in a large number of cases. The resolution of the L-lines in WDS is far better. Unfortunately, contributions to the detector efficiency of a crystal spectrometer cannot be calculated as easily as in EDS.

We therefore have developed a procedure for calibrating a lithium fluoride crystal spectrometer by using the $K_{\alpha}$ and $K_{\beta}$ lines for elements titanium through arsenic. The Cameca electron microprobe was used at a beam energy of $20 \mathrm{keV}$. A novel technique for recording WDS data was developed for this work. Using a multichannel analyzer (MCA) in the multichannel scaling mode, we made a very slow pass through each characteristic $x$-ray line. The MCA memory is sufficient to store a single $x$-ray line plus background on both sides of the peak. An example of a spectrum obtained in this way is shown in figure 33 .

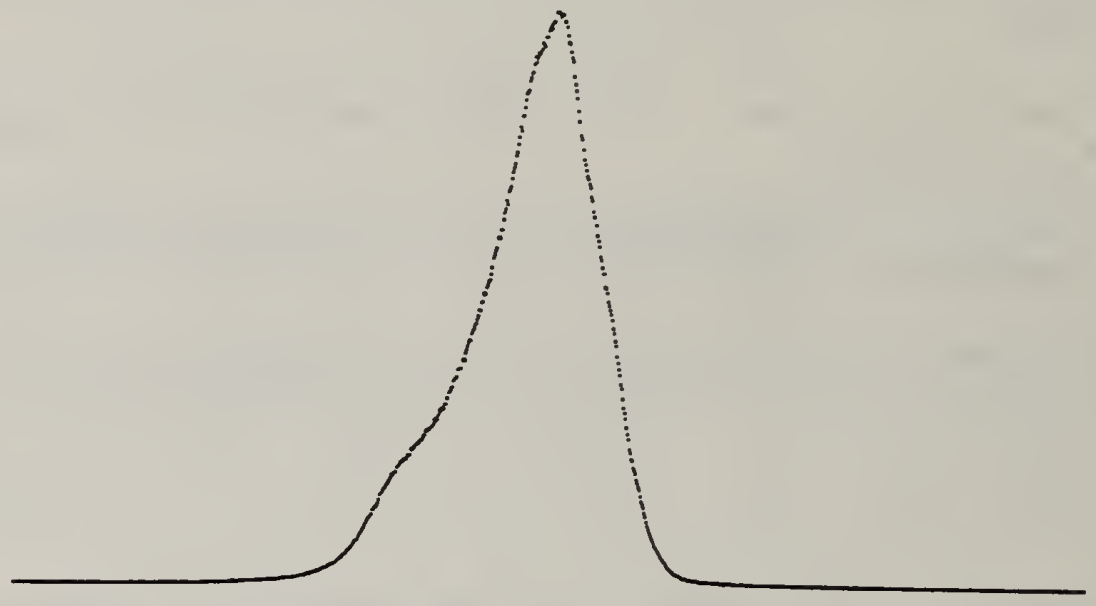

ENKA IX20

Figure 33. Wavelength spectrometer scan of Zinc $K_{\alpha}$ peak as recorded on digital multichannel scalar.

A ratio of the background-corrected integrated intensity of the WDS

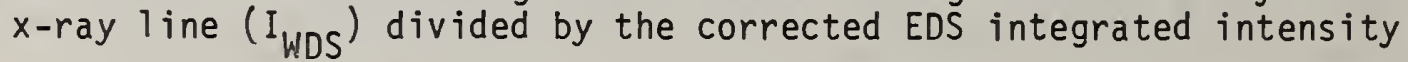
( $\left.I_{E D S}\right)$ for the same $x$-ray 1 ine was calculated. This ratio is expressed as $I_{W D S} /\left(I_{E D S} / P_{E}\right.$ ) where $P_{E}$ is the EDS detector efficiency correction (the sample absorption term cancels out as it appears in both numerator and denominator.) A plot of this ratio versus $x$-ray line energy gave a smooth curve as illustrated in figure 34 . This curve now can be used to make appropriate detector efficiency corrections to the $L-1$ ines detected 


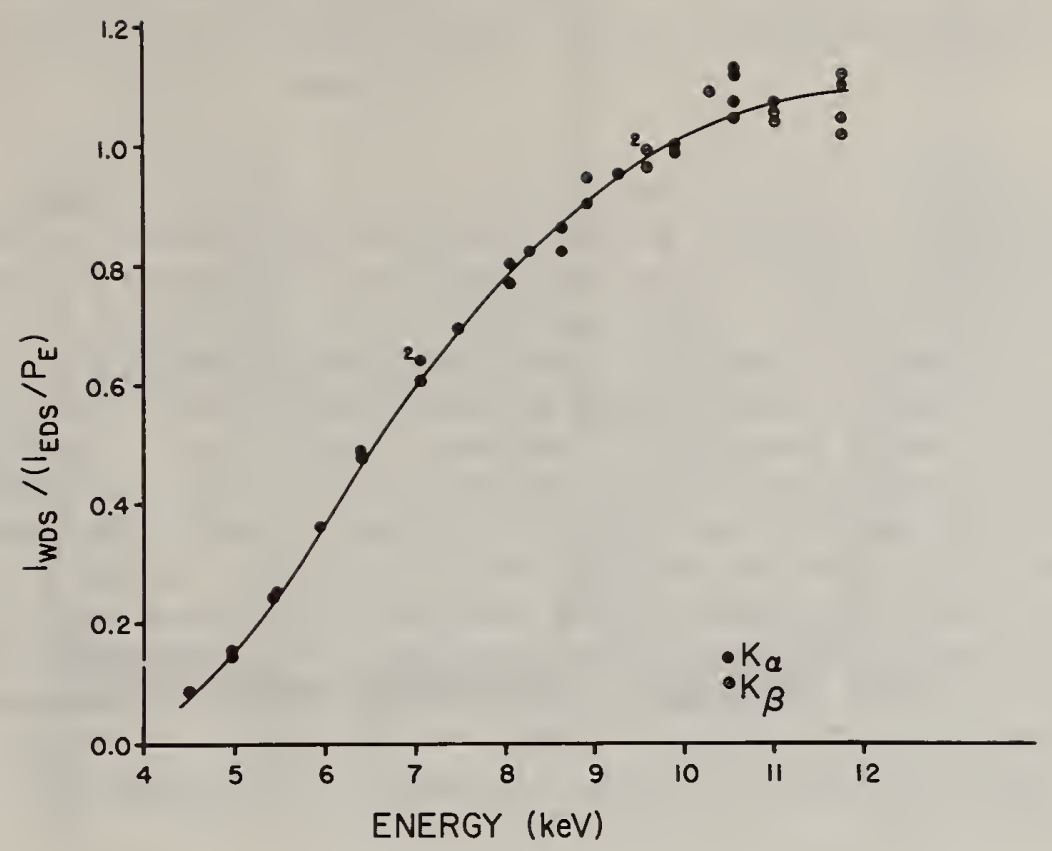

Figure 34. Plot of ratio of net integral intensities measured by wavelength and energy dispersive spectrometry techniques versus peak energy.

with the same crystal spectrometer at the same excitation potential. With this calibration, in addition to the overlap corrections, better relative line intensity data for L-lines should soon be available.

\section{References}

[1] Heinrich, K. F. J., Fiori, C. E., and Myklebust, R. L., Relative Transition Probabilities for the X-ray Lines from the K Level, J. Appl. Phys. 50, 5589 (1979).

[2] Fiori, C. E., and Myklebust, R. L., A Simplex Method for Fitting Gaussian Profiles to X-ray Spectra Obtained with an EnergyDispersive Detector, Proc. of the ANS Conf. on Computers in Activation Analysis and Gamma-ray Spectroscopy, Mayaquez, P. R., 1978 DOE Symposium Series, CONF-780421, p. 139.

R. Marinenko, R. Myklebust

15. Successful Raman Microprobe Analys is of Opaque Particles

The Raman microprobes now in use employ high intensity (from a few $\mathrm{kW} / \mathrm{cm}^{2}$ up to $1 \mathrm{MW} / \mathrm{cm}^{2}$ ) visible $\mathrm{CW}$ laser excitation to el icit 
diagnostically useful vibrational spectra from discrete microparticles or spatially resolved sampling volumes approaching one picogram in mass $[1-3]$.

While numerous applications have been successful, a major limitation of Raman microprobe analysis arises when the measurement is performed on intensely colored, highly opaque microsamples that absorb a large fraction of the exciting radiation. Dark materials (in the liquid or solid phase), until recently, produced unusable results because of laserinduced heating of the sample with attendant modification or decomposition even under the lowest practical irradiance conditions. The usual expedient of using an alternate excitation wavelength (e.g., choosing the 647.1 $\mathrm{nm}$ red 1 ine over the $488.0 \mathrm{~nm}$ blue 1 ine from an $\mathrm{Ar}-\mathrm{Kr}$ ion laser) and reducing the energy density focused into the sample often will neither greatly minimize nor circumvent the problems associated with sample heating from significant optical absorption. Thus, dark and opaque microsamples, without some means of effective heat dissipation, are either too weakly scattering or highly reflecting to furnish useful results, or yield spectra that are difficult to interpret due to sample tranformation.

To better cope with these experimental difficulties, special sample preparation techniques have come under study to permit obtaining the spectra of deeply colored microsamples.

One of the most successful methods consists of encapsulating particles, supported on a sapphire substrate, in a spectroscopically non-interfering dielectric film which efficiently enhances thermal sinking into the substrate. By embedment in a thin, glass-like layer of sodium silicate (i.e., water glass), the spectra of opaque particles have been recorded (with $514.5 \mathrm{~nm}$ green 1 ine excitation) where this was not possible before. Particle samples are coated with this encapsulating, glass film either by spreading of the liquid silicate or by forming the film through application of the silicate solution as a finely divided spray. The initially liquid film deposited by either of these procedures quickly dries to a hard, transparent film. In measurements on samples without the silicate overcoat, the mounting medium is air providing poor heat conduction across the particle/substrate interface. The spectra of figure 35 are those of opaque microparticles of black uranium oxide [4] - a most difficult scattering case - that could only be obtained by immersion in the silicate matrix. The top spectrum is that of a mixed uranimum oxide/ thorium oxide microsphere showing the expected Raman bands at the respective characteristic frequencies (i.e., at 445 and $467 \mathrm{~cm}^{-1}$ ). The bottom spectrum is that of a particle of synthetic, single-crystal uranium oxide also embedded in the silicate overcoat. Similarly, the spectra of single particles of many other types of highly absorbing solids (e.g., $\mathrm{Fe}_{2} \mathrm{O}_{3}$, $\mathrm{Cr}_{2} \mathrm{O}_{3}, \mathrm{~V}_{2} \mathrm{O}_{3}, \mathrm{~W}_{2} \mathrm{C}$ ) have been recorded using this method of sample preparation. The technique has further proven itself in microprobe studies on particulate samples of black carbon materials, including coal, oil-soot 


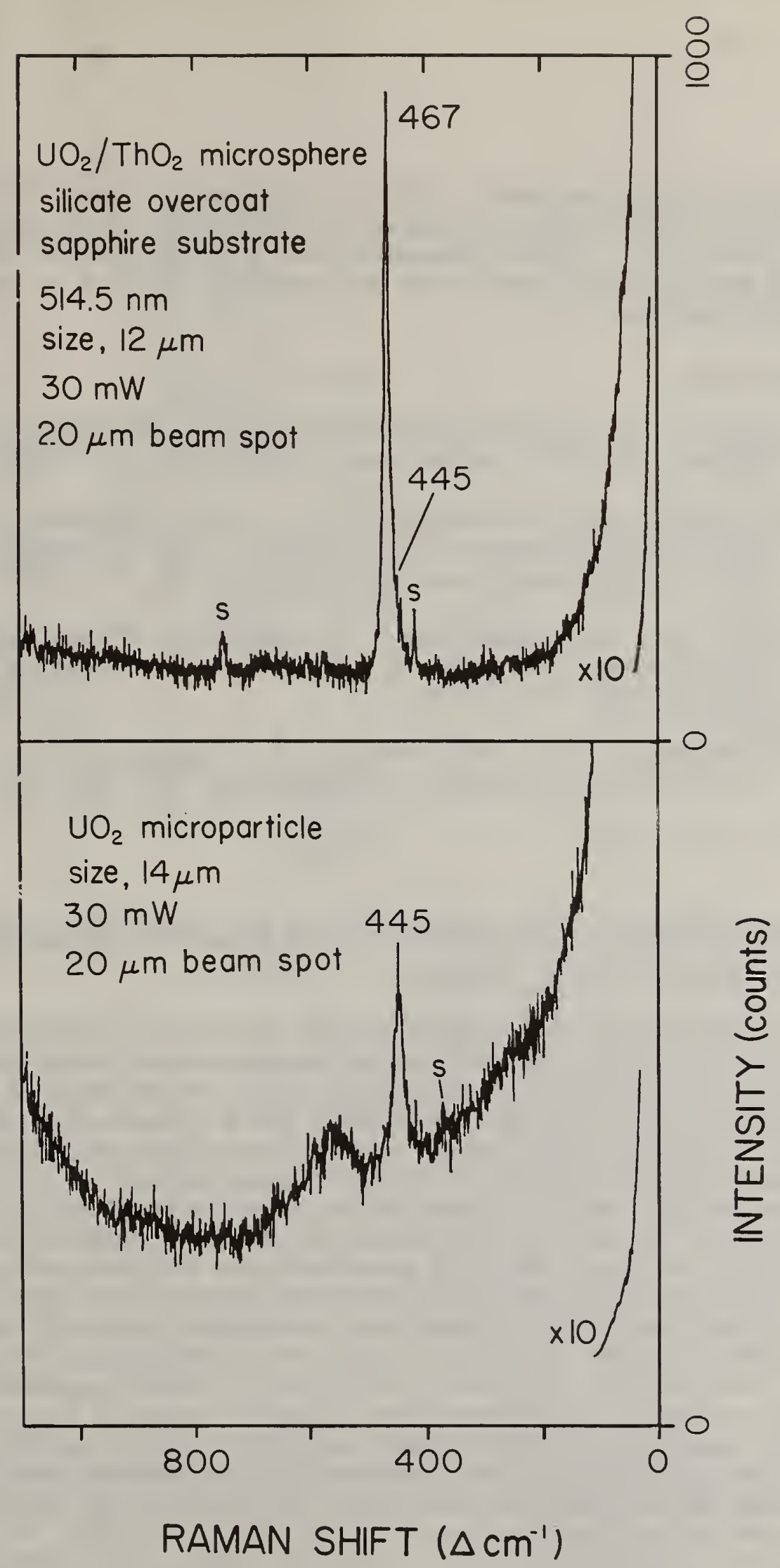

Figure 35. Raman microprobe spectra of uranium oxide particles embedded in a silicate film. Measurement conditions are indicated. Bands marked $\mathrm{S}$ arise from the Raman scattering by the sapphire $\left(\alpha-\mathrm{Al}_{2} \mathrm{O}_{3}\right)$ substrates. 
ash, and graphitized polymeric fibers. Intensely colored silicate minerals (e.g., crocidolyte asbestos) and absorbing, photosensitive organic solids (e.g., nitro compounds) have yielded spectra indicating effective thermal stabilization of the irradiated sample by immersion in the silicate matrix.

\section{References}

[1] Etz, E. S., Raman Microprobe Analysis: Principles and Applications, SEM/1973/I, SEM, Inc.: AMF 0'Hare, IL 60666, 67-82.

[2] Blaha, J. J., and Rosasco, G. J., Raman Microprobe Spectra of Individual Microcrystals and Fibers of Talc, Tremolite and Related Silicate Minerals, Anal. Chem. 50, 892 (1978).

[3] Abraham, J.L., and Etz, E. S., Molecular Microanalys is of Pathological Specimens in situ with a Laser-Raman Microprobe, Science 206, $716(1979)$.

[4] Keramidas, V. G., and White, W. B., Raman Spectra of Oxides with the Fluorite Structure, J. Chem. Phys. 59, 1561 (1973).

E. S. Etz, J. J. Blaha

16. Development and Characterization of a New Raman Microprobe With Multichannel Optical Detector

The Raman microprobes that have come into use in recent years employ CW laser excitation and basically make use of a scanning spectrometer (i.e., a monochromator) with conventional single channel (cooled PMT) detection [1-3]. These instruments yield analytically useful vibrational spectra from discrete microscopic objects (e.g., particles) or sample regions with dimensions of a few micrometers (e.g., inclusions). They have been applied with good success demonstrating detection limits lower than $1 \mathrm{pg}$ for scattering species in optically transparent sampling volumes [2]. Yet, microanalysis performed with these scanning instruments suffers from several shortcomings. Drawbacks result from primarily two factors. These are (1) the long (tens of minutes to hours) instrumentallyimposed time required to obtain a spectrum with monochannel detection, and (2) the often severe optical constraints of the measurement wherein the sample absorbs a fraction of the incident (visible) laser light leading to complications from sample heating or photochemistry. These problems can, in principle, be minimized or circumvented by microprobe instrumentation employing spectrographic dispersion with sensitive multichannel detection.

We have explored the advantages of this approach by developing (in collabcration with Instruments SA, J-Y Optical Division) a prototype Raman microprobe with multichannel optical detection (figure 36 ). This 


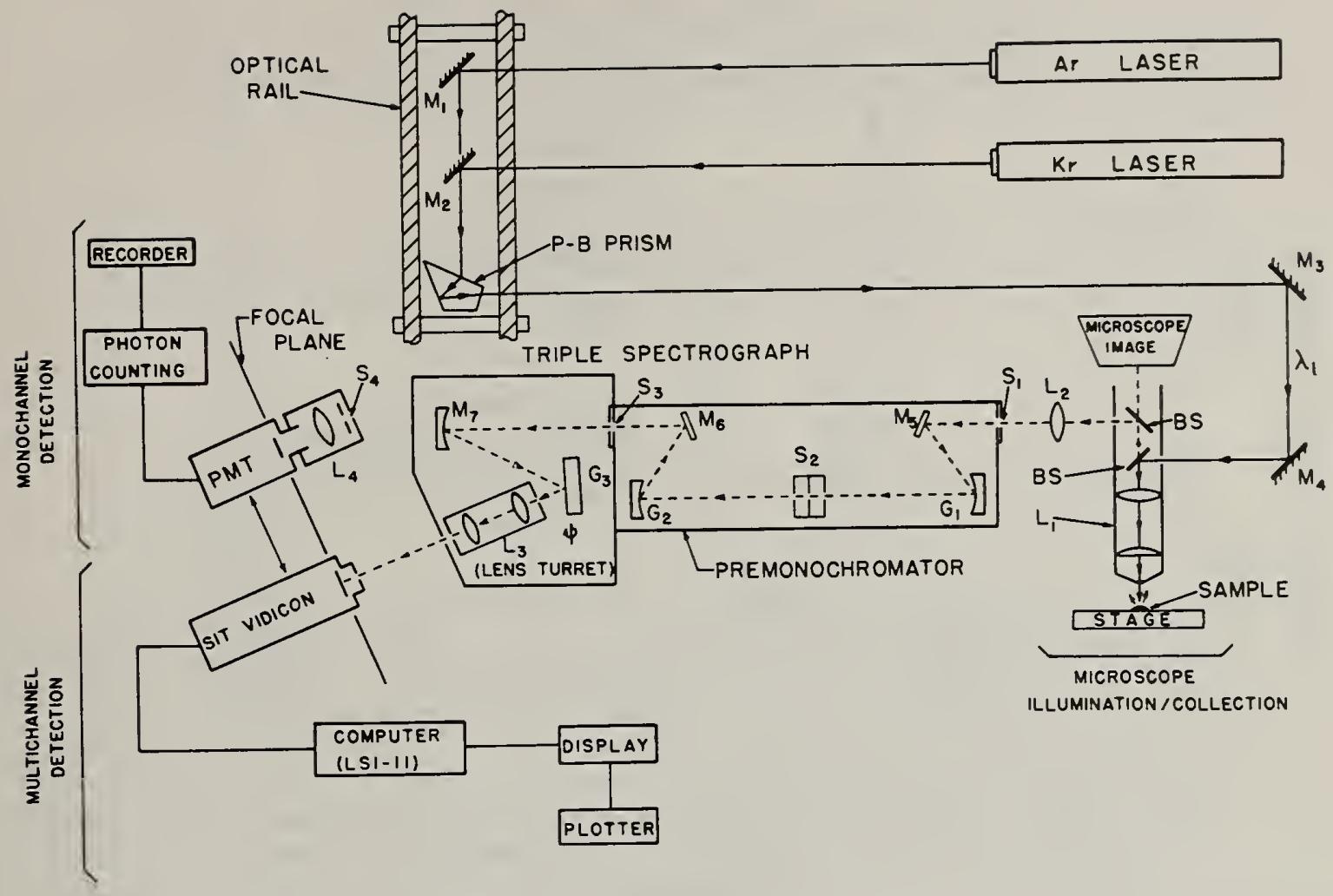

Figure 36. Schematic of the newly developed Raman microprobe.

new instrument has just become fully operational and is presently undergoing extensive performance characterization. Results obtained with this instrument demonstrate the spectral multiplex advantage in the analysis of "difficult" (e.g., optically absorbing or photosensitive) microsamples. This development has utilized commercially available equipment consisting of (1) two gas lasers ( $\mathrm{Ar}$ and $\mathrm{Kr}$ ion) as multiwavelength excitation sources, (2) a standard microscope for observation of the sample and excitation/collection of the scattered radiation, (3) a triple spectrograph of novel design for dispersion of the Raman spectrum, and (4) a multichannel detection system utilizing a cooled SIT vidicon. A separate, second detection system consists of a conventional photomultiplier tube (PMT) with photon counting electronics and permits scanning or spectrometric recording of spectra.

A spectrum from the measurement on single microparticles using the multichannel instrument is shown in figure 37 . The sample examined here is a microscopically small polymer latex particle. The experimental parameters are indicated. The spectrum was acquired in only three minutes of signal integration. This represents a time advantage in the acquisition of the spectral "fingerprint" of a factor of about 18 compared to the corresponding spectrum of comparable signal-to-noise obtained in a 


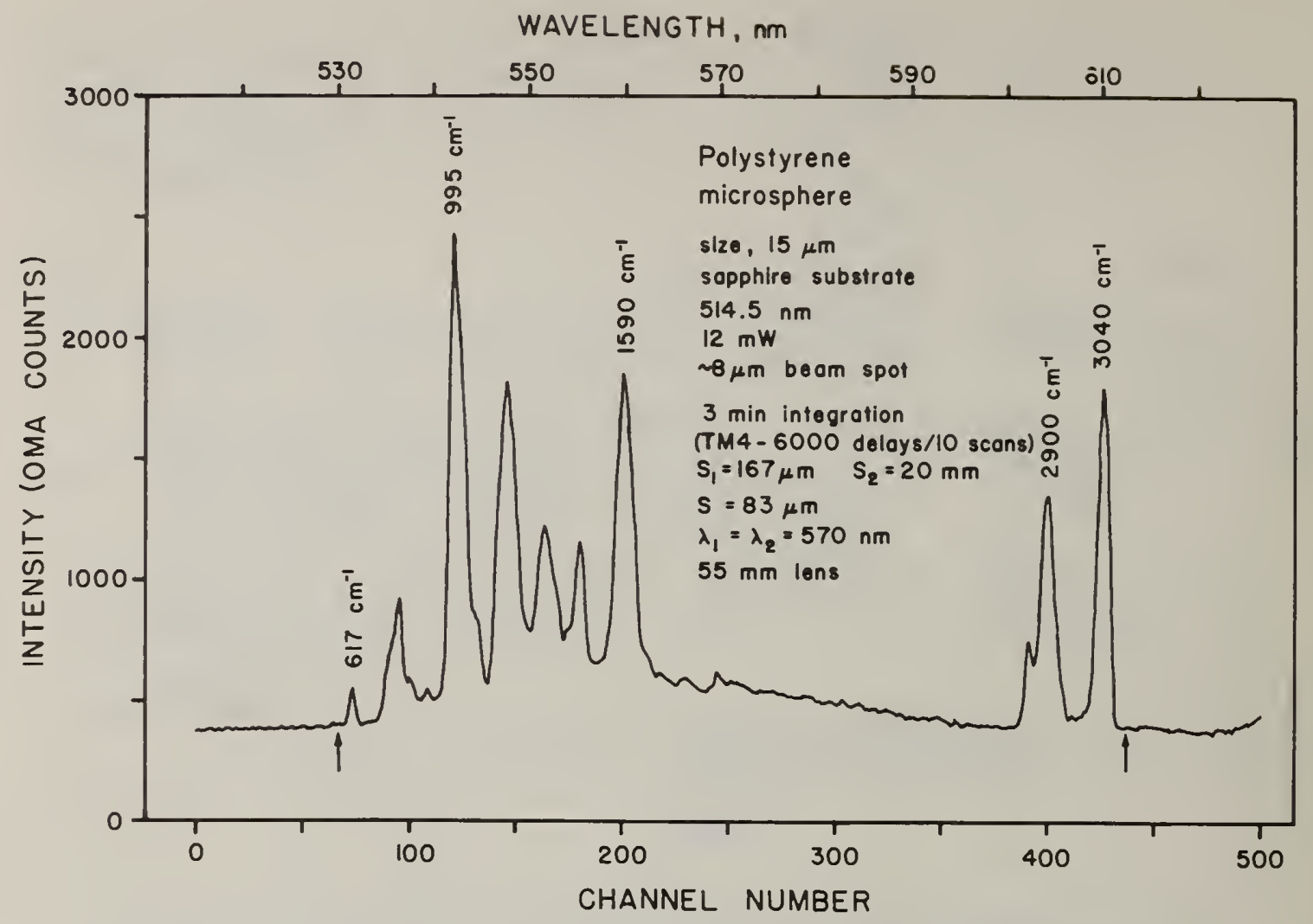

Figure 37. Raman spectrum of a latex microparticle obtained with multichannel detection.

conventional microprobe with a scanning spectrometer. Moreover, since the polymer is subject to time-dependent, slow decomposition even under conditions of moderate laser irradiance, these potentially adverse effects attributable to sample instability are in many cases not encountered and not observed in the example given here because of the fast, signal acquisition time.

The instrument was developed with support from the Air Force Technical Applications Center (AFTAC) and is now in use at an Air Force materials analysis laboratory on the West Coast.

Studies now in progress in cooperation with AFTAC are aimed at a more complete under-standing of the performance capabilities and intrinsic limitations of the new instrument. Current developments in radiation dispersion and detection systems are significant to further advances in this field of spectroscopic instrumentation.

References

[1] Dhamelincourt, P., et al., Laser Raman Molecular Microfrobe (MOLE), Anal. Chem. 51, 414A-421A (1979). 
[2] Etz, E. S., and Blaha, J. J., Scope and Limitations of Single Particle Analysis by Raman Microprobe Spectroscopy, in Characterization of Particles, NBS Spec. Pub7. 533, K. F. J. Heinrich, ed., U.S. Government Printing Dffice, Washington, D.C., 153-197 (1980).

[3] Rosasco, G. J., Raman Microprobe Spectroscopy, in Advances in Infrared and Raman Spectroscopy, Vol. 7, R. J. H. Clark, and R. E. Hester, eds., Heyden \& Son, Ltd., London, 223-282 (1980).

E. S. Etz

17. Ion Microprobe and Laser Microprobe Mass Analysis of Inorganic Particulates

Three microbeam instruments that use mass spectrometers as detectors have been investigated and compared with respect to the analys is of individual particles. Two of the instruments are based on ion sputtering. The ion microprobe uses a sputtering beam diameter as small as $2 \mu \mathrm{m}$ to obtain a localized analysis. The ion microscope, on the other hand, sputters with a considerably larger ion beam. However, in this case, sputtered ions preserve their spatial relationship through the mass spectrometer, and localization of the analyzed area is achieved by limiting the field of view of the detector with an appropriate aperture. The laser microprobe uses a pulsed laser beam that can be focused to a diameter of $1 \mu \mathrm{m}$ or less to vaporize and ionize a small volume of material. Since the ionization event is localized in time, a time-of-flight mass spectrometer is employed for mass analysis.

Samples used for this investigation were microchips and microspheres of research glasses manufactured at NBS. For the ion beam instruments the samples were dispersed on $0.5 \mathrm{~cm}$ diameter high-purity gold disks. For the laser microprobe they were dispersed on formvar-coated transmission electron microscope grids. The size range of the glass particles was 1$15 \mu \mathrm{m}$.

An example of comparative data from the three instruments is shown in Table 16. In this case the samples were spheres of a glass containing 80 percent $\mathrm{PbO}$ and 20 percent $\mathrm{SiO}_{2}$ by weight. The $\mathrm{Pb}$ and $\mathrm{Si}$ ion signals were measured from several particles of different sizes to determine the relative sensitivity of the two elements and its variability from particle to particle. The table indicates that the relative sensitivity differs by less than a factor of 2 among the three instruments in this case. The relative standard deviation (RSD) of the sensitivity ratio is highest for the laser microprobe. This result reflects a lack of reproducibility of ionization conditions among particles of different size as well as insufficient data density of two or three data points per mass peak when a wide mass range is recorded. 
Table 16. Lead - Silicon Sensitivity Ratios from K227 Microspheres.

$\begin{array}{lcccc} & \begin{array}{c}\text { Ion } \\ \text { Microprobe }\end{array} & & \begin{array}{c}\text { Ion } \\ \text { Microscope }\end{array} & \begin{array}{c}\text { Laser } \\ \text { Microprobe }\end{array} \\ \text { No. of particles } & 10 & & 5 & 8 \\ \mathrm{~S}_{\mathrm{Pb}} / \mathrm{S}_{\mathrm{Si}} & 2.13 & & 3.44 & 2.67 \\ \mathrm{SD} & 0.34 & & 0.24 & 2.14 \\ \text { Relative SD } & 16 & 7 & 80\end{array}$

Table 17 indicates the capability of the three instruments to measure isotopic ratios of $\mathrm{Pb}$ in these same microspheres. The higher precision of the ion microprobe measurements is attributable to an electrostatic peak-switching system that allows isotope ratio measurements to be taken without changing the magnetic-field setting of the mass spectrometer. The relatively low precision of the laser microprobe is a result of the 8-bit resolution of the transient recorder that is used to measure and store the mass spectrum in 256 discrete levels. This characteristic also restricts the dynamic range of signals that can be measured in one spectrum and sets a practical limit of about $10: 1$ on the magnitude of an isotopic ratio that can be measured with the laser microprobe. The ion microscope produces well-defined trapezoidal peaks that vary in intensity by 1 ess than 0.2 percent over the flat portion of the peak top. One would therefore expect the variation in isotopic ratio of a given element among a group of particles to be less than one percent, but that is not the case. A computer-controlled cyclic mass scan is used to measure the peak intensities in sequence for the isotope ratio calculation. Improvements in interparticle precision may occur through the use of a different software routine that minimizes the effect of magnetic hysteres is.

The true isotopic ratio of the $\mathrm{Pb}$ in these particles has not been measured, but the best estimate for naturally occurring $\mathrm{Pb}$ is shown in Table 17. Any bias in the measurement of the ratios in the particles could be corrected by comparison with a standard of known Pb isotopic composition. 
Table 17. Isotopic Ratios of Lead from K227 Microspheres.

\begin{tabular}{|c|c|c|c|}
\hline $\begin{array}{l}\text { Ion } \\
\text { Microprobe } \\
\end{array}$ & $\begin{array}{c}\text { Ion } \\
\text { Microscope }\end{array}$ & $\begin{array}{c}\text { Laser } \\
\text { Microprobe }\end{array}$ & $\begin{array}{c}\text { Natural } \\
\text { Lead }\end{array}$ \\
\hline 10 & 6 & 4 & \\
\hline 0.461 & 0.511 & 0.53 & 0.461 \\
\hline 0.6 & 3.4 & 15.4 & \\
\hline 0.412 & 0.457 & 0.47 & 0.422 \\
\hline 0.2 & 3.7 & 9.6 & \\
\hline 1.098 & 1.140 & $1.15^{\mathrm{a}}$ & 1.093 \\
\hline 0.011 & 0.005 & .09 & \\
\hline 1.0 & 0.4 & 7.8 & \\
\hline
\end{tabular}

$\overline{a_{13} \text { particles for }} 206 / 207$.

Table 18 summarizes some of the special features of each of the three instruments as applied to particulate analysis. It is apparent that no single instrument is the best choice for all situations. The ion microprobe can measure isotope ratios with high precision. The ion microscope has a variety of features that give it great versatility to handle different analytical problems, for example, the ability to screen a field of particles for a particular element by use of the ion-imaging mode of operation. Finally, the laser microprobe can acquire full mass spectra from many particles at a very rapid rate, and is the easiest instrument to use for the analysis of very small particles.

Table 18. Special Features of Three Instruments for Particle Analysis.

\section{Instrument}

Ion microprobe

Ion microscope

Laser microprobe

D. S. Simons

\section{Special Features}

High precision isotopic ratios

Rapid ion imaging of a particle field

Faraday cup or electron multiplier detector

High abundance sensitivity spectrometer

Molecular ion rejection by energy offset

High mass resolution

High resolution optical microscope

Rapid analysis (30-60 particles/hr.)

Ease of analysis of small particles (0.5-2 um) 
18. Evaluation of Counter Error in the Development of Asbestos Standards for the Electron Microscope

The Environmental Protection Agency (EPA) has been monitoring the concentration of asbestos in ambient air, because of its potential adverse health effects, using an electron-microscopic analysis of filtered air particulates.

During a number of interlaboratory studies, EPA found that the reported asbestos concentrations varied among laboratories by up to five orders of magnitude on the same filter. Many laboratories consistently reported relatively high or low results, in part due to operator bias or error, filter inhomogeneity and counting statistics. Generally, because of the high magnifications used during analysis, only approximately $1 / 100,000$ of the total filter area was observed. The data from this small area was then extrapolated to the whole filter to determine the relative concentration.

Because of these problems with asbestos analysis, EPA requested NBS to prepare filters with known asbestos fiber concentrations that are homogeneous on the level of a $\mu \mathrm{m}^{2}$. In attempting to approach as closely as possible the true asbestos fiber concentration on the filter NBS has used up to five microscopists to analyze the same areas of filters. These areas were then sent to other experienced laboratories for analys is. The results are shown in Table 19. The variability of these counts both with in NBS and at other laboratories was considered excellent by EPA. But these results could not be correlated to determine where the errors in analysis occurred. Also, it was felt that an average or mean value as reported in column 1 of Table 19 for the five NBS operators was not necessarily an accurate description of the number of fibers on the areas counted. For these reasons a digital specimen positioner was designed at NBS so that individual fibers could be uniquely identified by their $X-Y$ coordinates. Three to five NBS operators counted the same $75 \mu \mathrm{m} \times 75 \mu \mathrm{m}$ area, recording each fiber's $X-Y$ position. Then the counts were correlated and finally the area was reanalyzed to verify all fibers. In this way it was felt that the verified fiber count was as close as possible to the true concentration. Table 20 shows some of the data recently collected using this method. This table shows each operator's error as number of fibers counted and how many of these were incorrect counts, how many fibers were missed, and the verified concentration. 
Table 19. A Comparison of Asbestos Fiber Counts from Four Laboratories for Nine Areas. (Number of Fibers per $\sim 75 \mu \mathrm{m} \times 75 \mu \mathrm{m}$ area).

\begin{tabular}{|c|c|c|c|c|}
\hline Area & $\begin{array}{l}\text { Laboratory (NBS) } \\
\text { (Five Operators) } \\
\end{array}$ & $\underline{(2)}$ & (3) & (4) \\
\hline 1 & $32.6 \pm 2.5$ & 23 & 42 & 22 \\
\hline 2 & $31.8 \pm 1.6$ & 32 & 36 & 33 \\
\hline 3 & $27.6 \pm 1.5$ & 22 & 23 & 22 \\
\hline 4 & $15.8 \pm 1.5$ & 16 & 13 & 15 \\
\hline 5 & $49.8 \pm 4.4$ & 33 & 32 & 40 \\
\hline 6 & $57.0 \pm 2.3$ & 37 & 40 & 45 \\
\hline 7 & $53.2 \pm 3.7$ & 35 & 39 & 44 \\
\hline 8 & $53.0 \pm 3.5$ & 33 & 38 & 42 \\
\hline 9 & $39.0 \pm 1.6$ & 22 & 30 & 40 \\
\hline
\end{tabular}

Table 20. Operator Error Data for a $75 \mu \mathrm{m} \times 75 \mu \mathrm{m}$ Area Using a Verified Counting Technique Based Upon an NBS Designed Specimen Position Digital Readout.

Operator

Total Fibers

Fibers Not Counted

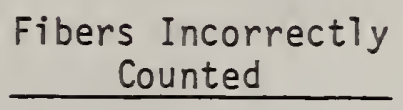

7

0

2

0
Verified Fibers Counted

26

26

26

26

Knowing the error associated with the five NBS operators and using the verified fiber counts allows work to proceed in characterizing fiber distribution and concentration of the final product, an asbestos filter standard.

E. Steel

19. The Development of Thin Glass Films As Potential Standards for X-ray jpectrometric Calibration"

$X$-ray fluorescence spectrometry has proven to be a versatile and rapid method for multielement analys is in many analytical applications. of particular interest are substances which can be analyzed in the form 
of a deposit on a filter, mesh or membrane, or as a thin pressed pellet. Collected airborne particulate matter, particulates in waste water and geochemical samples are just a few examples of this type. In some respects such "thin samples" are ideal for x-ray fluorescence analys is because the detection limits are suitably low and interelement effects such as $x$-ray absorption and/or enhancement are negligible. The absence of any sizable interelement effects makes possible a linear instrument response to element mass per unit area. Spectrometer calibration for analys is of such samples is usually performed with thin samples containing known concentrations of the analyte(s) of interest. Some problems associated with the "thin specimen model" are $x$-ray self-absorption in particles (notably for the light elements such as Al, Si, P, and S) commonly referred to as particle-size effects, and self-absorption in the substrate. Both of these effects require the application of correction factors. Therefore, the basic premise is that accurate multielement analysis of thin samples can be performed based on the thin specimen model, together with empirical or theoretical correction factors.

There are several important criteria which need to be met if thin samples are to be acceptable for calibration purposes. First, the uniformity of the sample as well as its homogeneity must be within an acceptable well-defined tolerance limit. If the sample contains particulate matter, both the size and distribution should be characterized. Secondly, the mass loading of the sample must be known to an acceptable degree of accuracy. And finally, the sample should possess sufficient durability and integrity.

The goal of a joint program between NBS and the U.S. Environmental Protection Agency (EPA) was to perform the research and development required to produce thin standard samples especially useful for calibration of x-ray fluorescence analysis of collected airborne particulate matter. For this purpose we have investigated several candidate methods for preparing such samples.

One method which shows great promise is the deposition of thin films of glass containing known concentrations of several elements of interest on polycarbonate substrates by a technique known as focused ion-beam sputtering. Ion-sputtering essentially involves the transfer of atoms and/or molecules from a target to a substrate by the action of an ion beam (usually argon) incident upon the target. The ion beam is accelerated at high potential (700-1100 volts with a flux of about $0.5 \mathrm{~mA} / \mathrm{cm}^{2}$ toward the target at a $45^{\circ}$ angle. Since the substrate is not in the plasma environment, substrate damage does not occur. This is a distinct advantage of this particular sputtering technique. Other advantages to be gained are: (1) production of thin films from synthetic materials such as glasses of known elemental composition which can be varied as desired; (2) high adherence of the glass to the substrate which obviates the need for overcoating to maintain sample integrity; and (3) easy control of the glass mass loading on the substrate by variation of the sputtering time. 
Several studies were performed with glass targets fabricated at NBS which allowed up to eight $47 \mathrm{~mm}$ substrates to be coated at one time. Instrumental parameters such as ion acceleration voltage and ion current were systematically varied to determine their effect on film composition. It was found that rather severe changes in these parameters often do not affect the film composition by more than \pm 15 percent. Glass films sputtered from targets containing phosphorous and sulfur were also prepared to demonstrate the feasibility of preparing films containing elements of low atomic number.

In Table 21 are summarized the elemental composition of three sets of films at mass loadings ranging from 167 to $185 \mu \mathrm{g} / \mathrm{cm}^{2}$ which were prepared at various times. These data demonstrate that the compositional reproducibility of thin films from set to set is satisfactory for the purposes of preparing standard films.

Multi-element thin standards suitable for calibration purposes can be fabricated by the focused ion-beam sputtering technique. In order to include all the elements of interest in any particular film, several glass targets would need to be fabricated. It is believed that these films would be useful to monitoring agencies such as EPA for assessing environmental impact of airborne particulate matter. Work is now in progress to prepare several sets of films which will be certified by the Office of Standard Reference Material as NBS Standard Reference Materials.

P. A. Pella, E. C. Kuehner, J. Sieber 


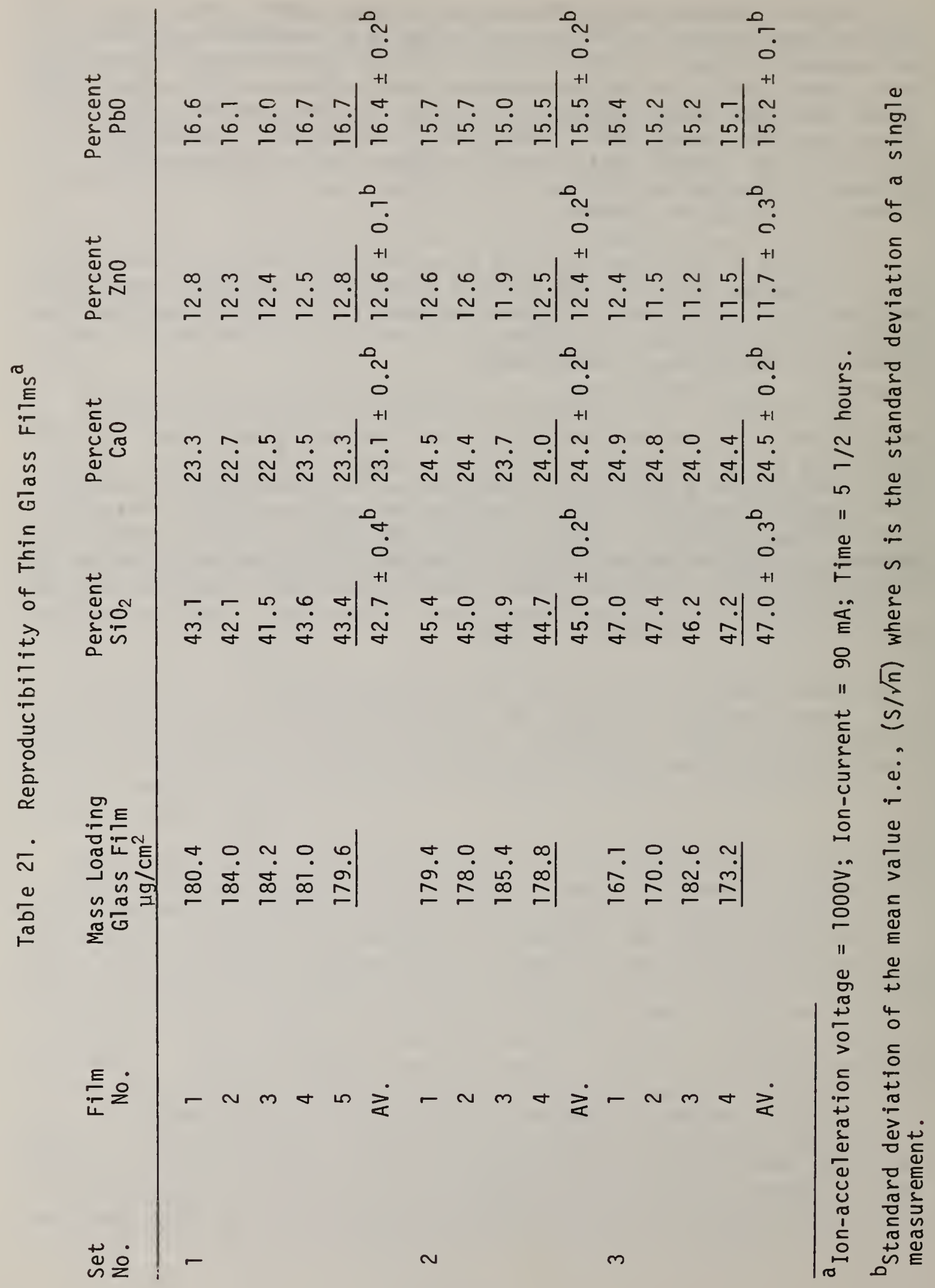


20. Low Velocity Wind Tunnel Facility for the Evaluation of Personnel Dosimeters

A low velocity wind tunnel has been designed, constructed and characterized. The tunnel enables us to produce aerosol and gas samples of known component concentration and flow profiles for evaluation of the efficiencies of aerosol and gas sampling devices. The tunnel consists of a high efficiency absolute filter at the inlet, followed by a mixing grid to flatten the flow profile and an injection section for the introduction of aerosol or gas samples. The test or sampling section of the tunnel (12 feet downwind of the injection section) has a 2-dimensional automated traverser which enables us to accurately position and locate velocity or sample probes in order to measure the velocity and sample concentration profiles in the tunnel. The maximum velocity is $\simeq 3 \mathrm{~m} / \mathrm{s}$. The velocity profile is flat to better than 10 percent in the cross-section of the tunnel beyond the 2 inch wall boundary layer. There is very low mixing in the tunnel so that when a point-source injection system is used, we obtain sample profiles that are quite steeply parabolic. For multiple sampler testing we will need to use either multiple injection or more severe mixing to broaden the sample concentration profile.

We have used the wind tunnel to evaluate a personal ambient aerosol sampler (PAAS) that is currently under development at NBS. The two components of the PAAS--the aerosol sampling/collection section and the sampling pump--have been evaluated separately. Eight different pumps have been evaluated for power requirements, pumping efficiency and flow stability. A pump has been selected that provides 5-6 liters per minute for periods greater than the desired sampling period of 8 hours and has low power consumption (Bendix BDXX55). The pump was tested under load conditions on the sampler that exceed loading conditions normally found in field applications.

The aerosol sampler selected is a two stage series filter system using a $37 \mathrm{~mm}$ diameter, $8 \mu \mathrm{m}$ nucleopore filter followed by a high efficiency teflon filter. The sampler is to divide and collect ambient aerosol in two size fractions. The first filter ( 8 um nucleopore) will collect respirable particles (about 2 to $15 \mu \mathrm{m}$ diameter) and the second filter (teflon) will collect the remaining smaller particles. The cutoff size (particle size at which the filter is 50 percent efficient) was determined for the nucleopore filter using monodisperse aerosols generated by a vibrating orifice aerosol generator. Fluorescein aerosol was used to enable accurate spectrophotometric determination of low aerosol concentrations on the filters. The filter collection efficiency for the nucleopore filter is presented in figure 38 as a function of particle aerodynamic diameter where aerodynamic diameter is a parameter that normalizes particles (regardless of shape) in terms of aerodynamic behavior for a unit density sphere. The collection efficiency for the first filter is defined as the aerosol concentration on the first filter divided by the sum of the concentrations on the first and second filter (total aerosol collected by the sampler). The sampler has the desired 


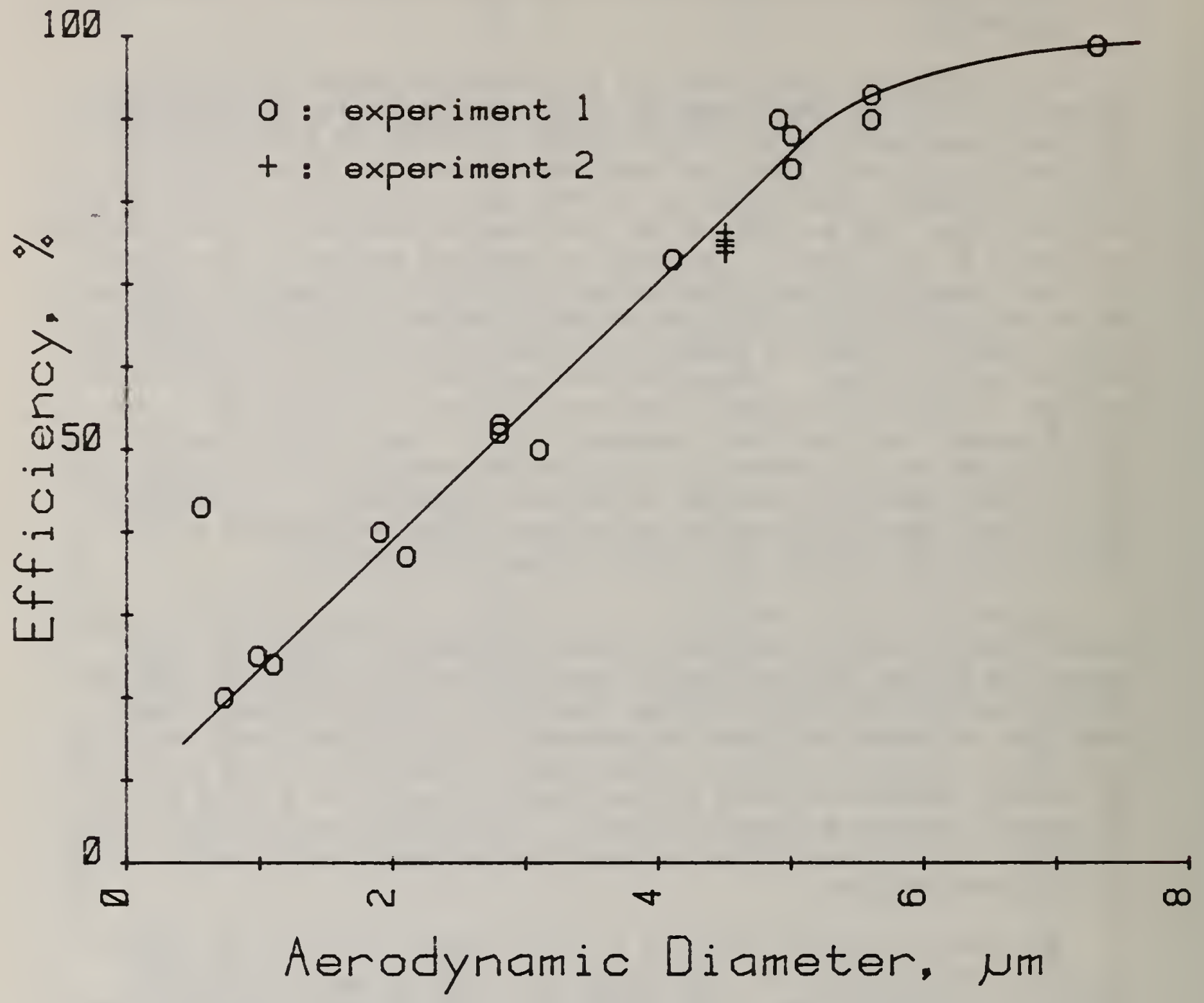

Figure 38. Coarse filter collection efficiency vs. particle size.

cut-off point of 2-3 $\mathrm{m}$ which does not vary significantly with flow rate in the range from 4 to 6 liters per minute.

R. L. McKenzie, D. S. Bright, R. A. Fletcher

21. Enhanced Detection of Molecules Near a Surface by Raman Spectroscopy Excited by Evanescent Waves

Raman spectroscopy is an increasingly important technique for specific detection of molecules. Its use has been severely limited in detecting and identifying small numbers of molecules by the extremely 
weak signal that usually results from Raman scattering. Recently a greatly $\left(10^{4}-10^{6}\right)$ enhanced signal has been observed for many types of molecules adsorbed in vacuum and bound in solution to an electrode surface for some metal surfaces. This surface enhanced Raman spectroscopic (SERS) signal has been used to study the identity, surface bonding character, and orientation of adsorbed molecules.

In two related experiments performed at Yale University (with Professors R. K. Chang and R. E. Benner in the Department of Engineering and Applied Science), we examined the Raman spectra produced by molecules at or near (within 1000A) the surface of a prism. A hemicylindrical prism with a 500A layer of evaporated silver on the flat face was oriented with the flat face toward the Raman spectrometer. Molecules of interest were placed on the flat side (see figure 39) and a laser beam was directed through the curved surface at angles with respect to the flat face equal to or greater than the critical angle for total internal reflection of that incident beam. The electromagnetic field which oscillates at optical frequencies, does not propagate from the prism into the less optically dense material (i.e., the sample), but does produce an oscillating electromagnetic field, called an evanescent field, which decays exponentially in amplitude with distance from the interface. The evanescent field decays to $1 / e$ of its value at the interface in 100-200 $\mathrm{nm}$. Evanescent excitation was used in our experiments to investigate the role in SERS of collective excitation of electrons (surface plasmons) in a silver film on the prism surface. A paper which discusses the one order of magnitude enhancement of Paman scattering by surface plasmons in SERS is soon to be pubT ished in Surface Science. A second paper (in preparation) describes the first use of Raman spectra as a probe of the orientation of the evanescent field with respect to the surface with and without surface plasmon enhancement. This new data and associated calculation will provide a basis for useful measurement of molecular orientations near an interface. 


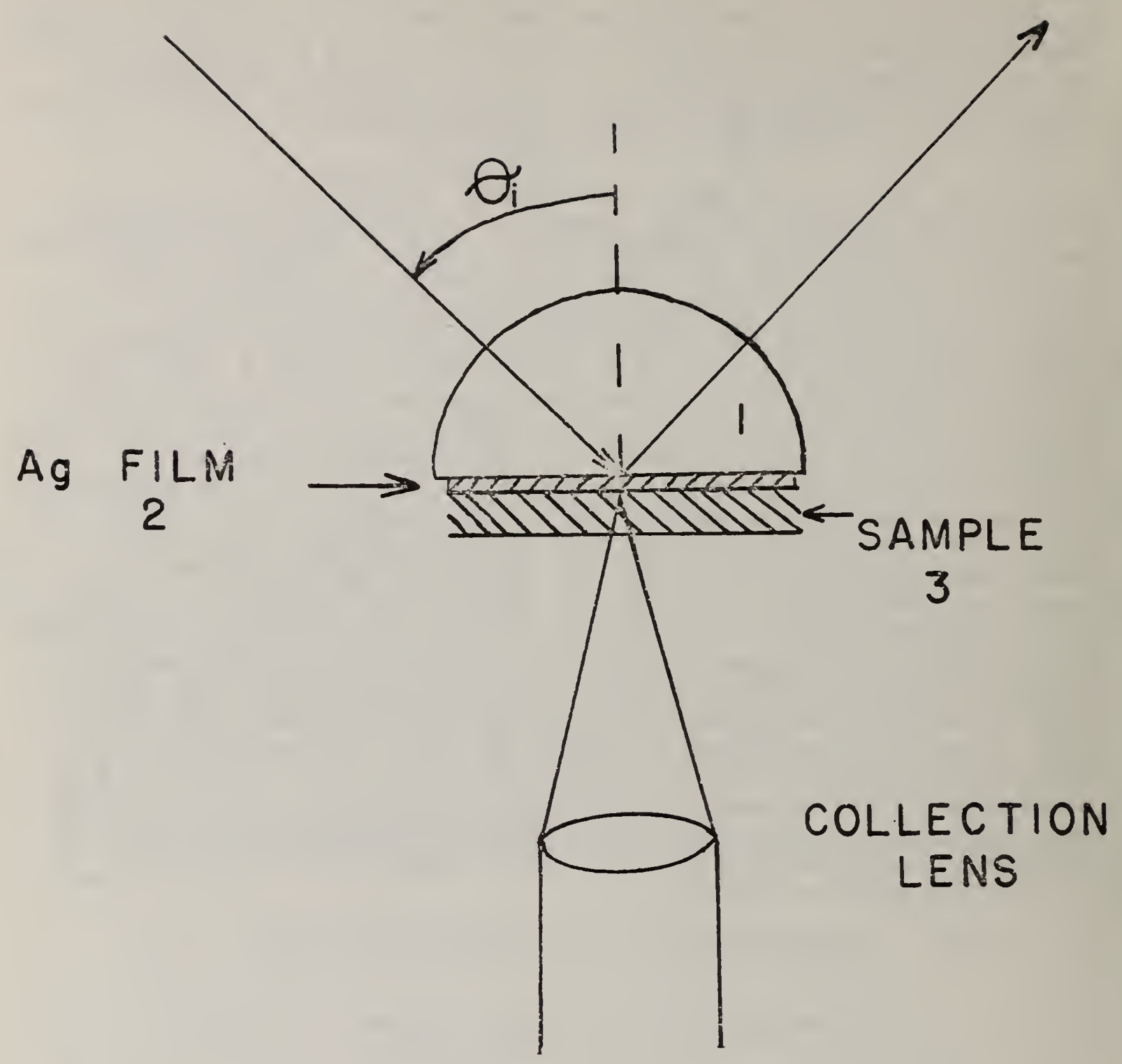

Figure 39. Geometry for evanescent wave-excited Raman Spectroscopy.

I. Chabay

22. Statistical Evaluations of the International Radiocarbon Cross Calibration Exercise, and of the Natural Radiocarbon Fluctuations

High accuracy natural radiocarbon measurements are of critical importance in a number of fields, ranging from archeology to climatology to environmental science. In addition to our current activities related 
to the calibration of the new International Standard for Radiocarbon Dating (replacement for SRM 4990B), we have contributed to the numerical evaluation of interlaboratory data representing a) high precision measurements of homogeneous primary and secondary natural radiocarbon standards, and b) the "calibration" relationship between radiocarbon concentrations and calendar age as deduced from dendrochronology (tree ring measurements). The first activity is important for assessing the relative activity of the three "standards" and for identifying error sources and limits (random and systematic) among the best laboratories internationaliy. Deliberations on the second topic culminated in the organization of the International Committee for the Calibration of the Radiocarbon Time Scale, to which NBS scientist, Lloyd A. Currie, is an advisor. The fine structure of the radiocarbon dating calibration curve -- i.e., long-term trends and short-term fluctuations about the simple exponential decay model -- is important for two major reasons: a) fine structure must be adequately evaluated/fitted to achieve accurate dating, b) modeling of the fine structure provides a means for us to learn more about the history of the earth (climate, environmental changes, ocean circulation, solar activity, etc.).

a. Exploratory Analys is of the International Radiocarbon Cross-Calibration Data: Consensus Values and Interlaboratory Error

Data from the international cross-calibration exercise have provided an important opportunity to assess the state-of-the-art of high-precision natural radiocarbon measurement and to derive consensus values for two relatively pure and homogeneous materials relative to the international radiocarbon dating standard (NBS Oxalic Acid, SRM-4990B). Performance was good: some fifteen laboratories returned results for the two independent ratios -- Australian National University Sucrose/Modern Standard (S/M) and 1850 Wood/Modern Standard (W/M) -- with a typical imprecision (standard deviation) of about five percent per mil; and the distribution of results was relatively narrow. The primary limitations encountered were incomplete laboratory data and variable reporting practices.

Analysis of the data led to the following observations:

- Imprecisions (standard deviations) reported varied from about two percent per mil to eight percent per mil, with one laboratory reporting 28 percent per mil. Most, but not a 11 , imprecisions represented counting statistics only.

- Apparent blunders (large deviations from consensus values) occurred in a few cases, especially for estimates of the ratio $S / M$.

- "Laboratory error," $\sigma_{x}$-- i.e., a non-Poisson error component -was estimated from the data distributions for both sample ratios. Results were similar: for $S / M, \hat{\sigma}_{x}=3.5$ percent per $\mathrm{mil}$; for $W / M, \hat{o}_{x}=1.8$ percent per mil. These error estimates, though 
rather uncertain because of the limited number of degrees of freedom, are consistent with earlier estimates, and they may reflect the accuracy 1 imit for current counting techniques.

- Consensus ratios, following the use of exploratory techniques to identify blunders, and taking into account "laboratory error" and varying Poisson imprecision, were $\triangle^{14} \mathrm{C}=508.1 \pm 2.0$ percent per mil (1850 Wood, age-corrected for decay to 1950). (Uncertainties represent one standard error, and both $S$ and $W$ are normalized to $\delta^{13} \mathrm{C}=-25$ percent per mil.)

Further results of the evaluation include $\delta^{13} \mathrm{C}$ distributions and the search for measurement method-, sample nature-, and isotope fractionationrelated effects. (See figure 40 for histograms of the intercomparison results.)

\section{b. Calibration of the Radiocarbon Time Scale}

Evaluation of the structure of the radiocarbon calibration curve is critically important both for accurate dating (as shown in figure 41) and for geophysics and climatology (figure 42). At the present time, because of the remarkable precision of natural radiocarbon measurements and the unique time ( 8000 year) record provided by dendrochronology, radiocarbon is the only radionuclide capable of giving us direct information on past fluctuations in solar activity. When combined with geophysical modeling and ice core measurements of the ancient atmosphere $\left(\mathrm{CO}_{2}\right)$, the ${ }^{14} \mathrm{C}$ variations will yield further insight into the history of the carbon cycle and prognosis for "the $\mathrm{CO}_{2}$ problem." 


\section{CROSS-CALIBRATION HISTOGRAMS}

(corrected ${ }^{14} \mathrm{C}$ deviations, in \%o)

\section{S/M}

\begin{tabular}{|c|c|c|c|c|c|}
\hline $\begin{array}{l}530 \\
525\end{array}$ & 9 & \multicolumn{2}{|c|}{ W/M } & \multicolumn{2}{|c|}{ S/W } \\
\hline 520 & & 10 & $s$ & 525 & 9 \\
\hline 515 & $s$ & 5 & $q$ & 520 & $\mathrm{G} \mathrm{km}$ \\
\hline 510 & A G k & 0 & d j L & 515 & 0 \\
\hline 505 & E F'L 0 & -5 & $A B E G h$ & 510 & $A E F$ \\
\hline 500 & B d $\mathrm{m} \mathrm{R}$ & -10 & F $k$ k & 505 & $\mathrm{~B}$ \\
\hline 495 & h j & -15 & $\mathrm{mP}$ & 500 & $d h L$ \\
\hline 490 & & & & 495 & c j P s \\
\hline 485 & $I$ & & & & \\
\hline 480 & $P$ & & & & \\
\hline
\end{tabular}

- S, M, W = Sucrose, Modern Standard, Wood

- $\sigma_{i}=2 \% 00-4 \%$ (Cap.); 5\%00-8\% (Lower Case)

Figure 40. Histogram of the radiocarbon ratios for ANU sucrose (S), 1850 wood $(W)$ and the modern standard ( $M$; $0.95 \times$ NBS oxalic acid). Numerical scales are expressed as deviations (in parts-perthousand) from unity from each of the three ratios; and laboratories are coded alphabetically. 


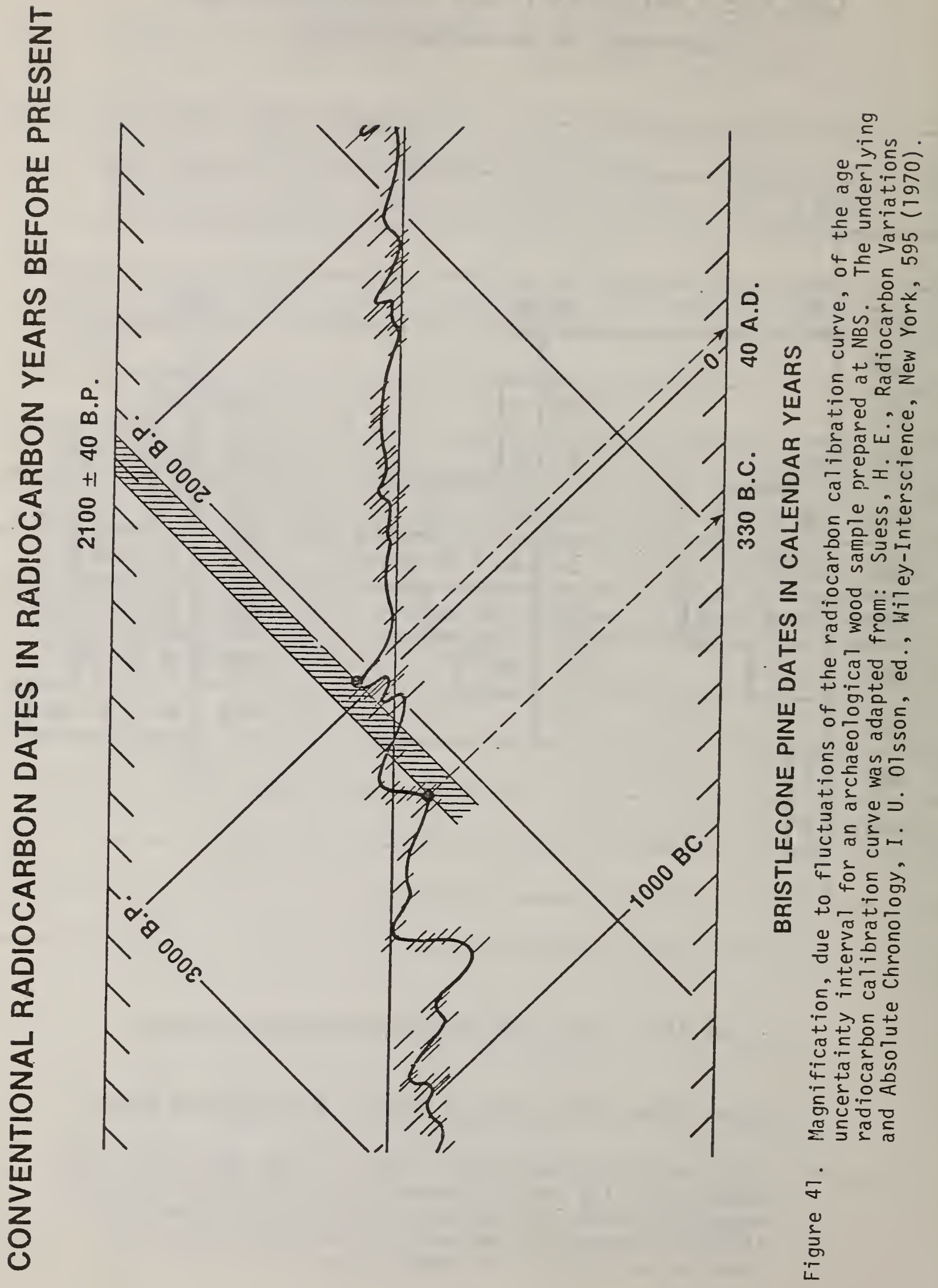



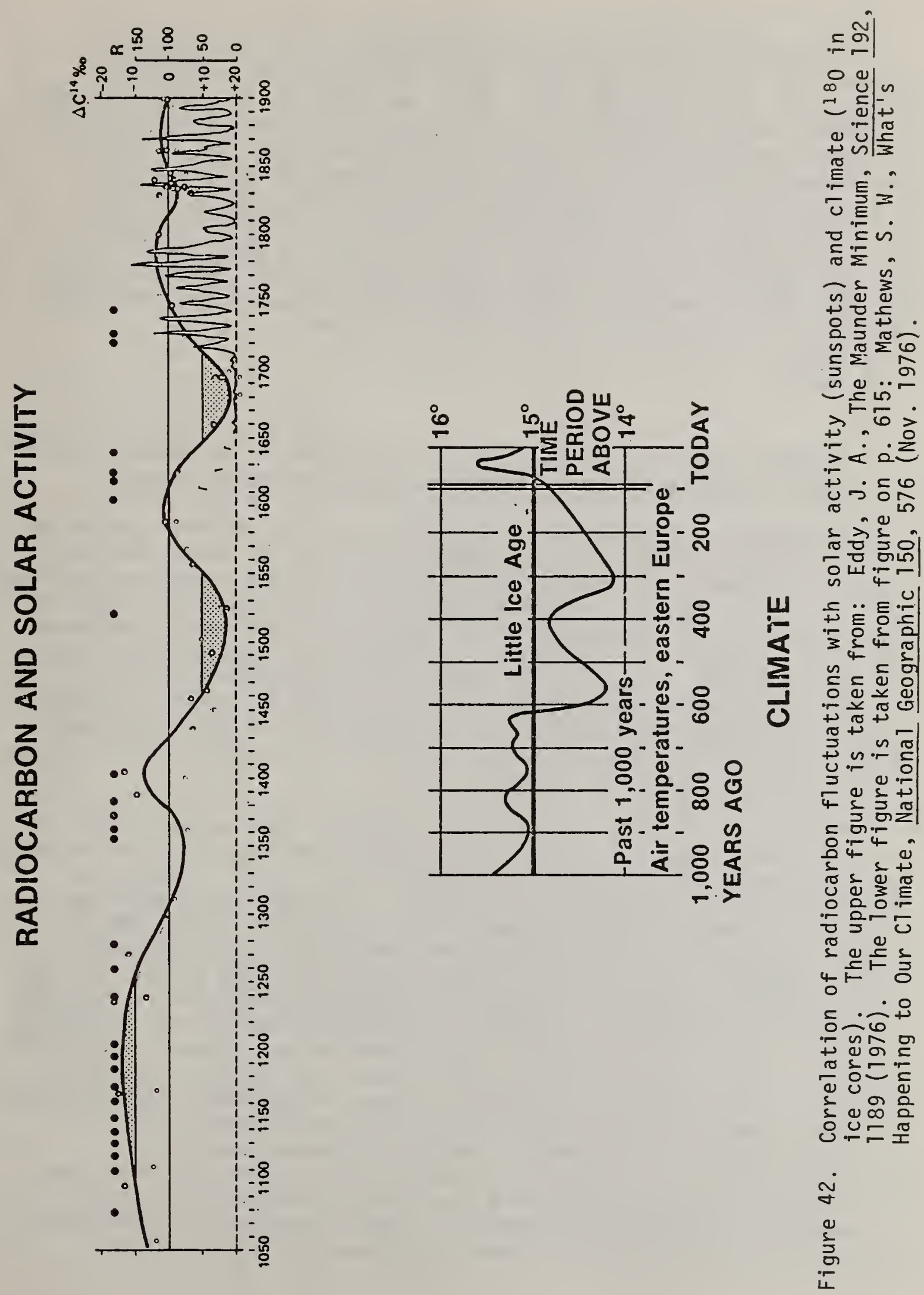
Objectives of the International Committee are to assemble high quality data and to utilize geophysical and statistical-mathematical approaches to "fitting" the data. More specifically, the Committee seeks to identify means for combining all (reliable) ${ }^{14} \mathrm{C}$ measurements of treering dated wood specimens into a single radiocarbon scale calibration scheme, preserving as much of the high frequency components as seem warranted by the data. During the past year cooperative efforts by L. A. Currie (Center for Analytical Chemistry), C. Spiegelman (Center for Applied Mathematics), and J. C. Lerman (Univ. of Arizona) have produced important insights into measurement and interlaboratory errors (see also part 1, above), assumed functional models, and best estimates for the calibration curve and its uncertainty band.

L. A. Currie

23. Advances in Small Sample Radiocarbon Measurement Techniques; Application to the Assay of Individual Atmospheric Chemical Species

During the past year the NBS program to utilize natural radiocarbon as a tracer for sources of carbonaceous pollutants has been directed toward a) fundamental advances in measurement and data evaluation techniques and b) critical studies of urban air pollution episodes (such as the Denver "brown cloud") and basic investigations of sources of atmospheric gases and particles. The importance of these investigations rests upon the impact of both natural and man-made carbonaceous contaminants on health, stratospheric ozone, and climate. The success of this program, the only such program extant, is due to the ability of NBS to perform reliable radiocarbon measurements on ambient samples containing microgram to milligram quantities of carbon.

Measurement advances are continuing through the development of miniature gas proportional counter and nuclear accelerator ("atom-counting") techniques which provide sensitivity gains of $10^{2}$ to $10^{5}$ compared to conventional radiocarbon dating techniques. Important meetings treating these topics (among others), organized by individuals in the group, included an ACS Symposium ("Nuclear and Chemical Dating Techniques", March 1980) and a meeting at NBS (August 1980) on sample preparation for accelerator measurements. Our work with the accelerator, which last year demonstrated the capability of precise measurement using less than $40 \mu \mathrm{g}$ (carbon) of the new international radiocarbon dating standard, is continuing with studies in alternative source preparation methods, isotopic enrichment, and baseline tests with the first "dedicated" tandem accelerator constructed exclusively for atom-counting measurements. This particular machine is considered a National Science Foundation Regional Facility, and it will be located at the University of Arizona. The accelerator approach will make possible the radiocarbon assay of $\mu \mathrm{g}$ quantities of individual hydrocarbons and halocarbons, and individual chemical and size fractions of non-urban atmospheric particulates. 
Applications to urban pollution and source studies of carbonaceous particles have centered on vegetative burning in the Portland, Oregon region, vegetative vs. oil shale aerosol contributions in a remote Utah location, urban pol1ution in Los Angeles and Denver, and an international effort (joint US - USSR AFAEX-79 project) to investigate "processes and mechanisms of formation and transformation of natural aerosol" in a remote forested region in the Soviet Union. In all of these studies, which were broadly interdisciplinary in nature, our group provided the only isotopic data; and the natural radiocarbon results yielded the only unique measure of the biogenic (non-fossil) contribution. This was especially important in the Denver and Portland studies where as a result of our measurements it was concluded that residential wood burning constituted one of most significant sources of inhalable $(<2.5 \mu \mathrm{m})$ and visibility-reducing particles. In Denver for example, it was concluded that 18 percent of the visibility reduction of the winter time atmosphere arose from wood burning. Furthermore, 39 percent of the most effective visibity-reducing species -- elemental ("graphitic") carbon -- has been ascribed to wood burning.

It should be noted that the foregoing regional studies have included considerable data on chemical and physical characteristics of the ambient particles, together with supporting emission inventory and meteorological data. Although radiocarbon techniques provided the only direct measure of contemporary carbon, the other (indirect) indicators supported the conclusions from radiocarbon measurements in every case. A major challenge exists in selecting and evaluating the entire multidimensional data patterns from such experiments; this need formed the basis for the first Receptor Modeling Workshop (March 1980 in Durham) and the resulting committee on chemical mass balance and multivariate analysis methods of source reconciliation. Our program at NBS has been expanded to include such modeling efforts, in cooperation with NBS Center for Applied Mathematics. The combined use of isotopic data $\left({ }^{13} \mathrm{C}\right.$ and $\left.{ }^{14} \mathrm{C}\right)$ with particle size and chemical data (pyrolysis mass spectrometry, inorganic composition and elemental vs. organic carbon) has, in fact, been an intrinsic part of the above urban and remote particulate studies.

A summary of our recent radiocarbon measurements, which now include atmospheric gases as well as particles, is given in Table 22. Future work will be directed toward further sampling of selected gases (hydrocarbons, carbon monoxide), sources of urban run-off and sediment studies, high-altitude sampling, investigations of arctic haze and climatological consequences, and paleoatmospheric (isotopic) composition via ice core samples.

L. Currie, G. Klouda 


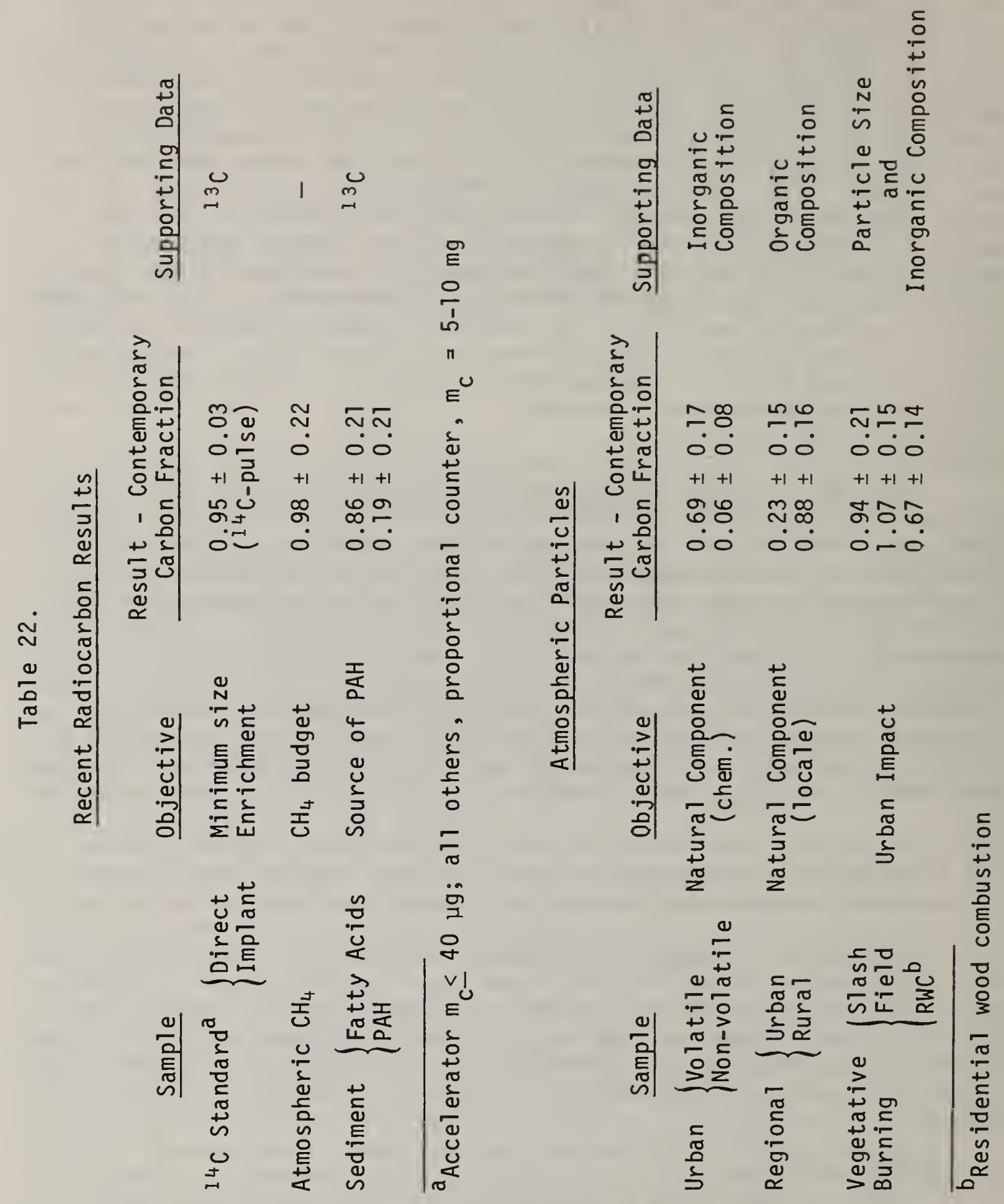


C. Outputs and Interactions

(Gas and Particulate Science Division)

1. Publications

Rosasco, G. J. and Blaha, J. J., "Raman Microprobe Spectra and Vibrational Mode Assignments of Tale," Applied Spectroscopy, Vol. 34, No. 2, October 1979, p. 140-144.

Blaha, J. J. and Etz, E. S., "Some Experimental Problems in the Raman Analysis of Microsamples," Proceedings of the 15th Annual

Conference of the Microbeam Analysis Society (San Francisco Press, 1980) p. 183-184.

Blaha, J. J., "Trace Characterization of Drugs of Abuse," NBS Dimensions, 64(3), 18-19 (1980).

Bright, D. S., Fletcher, R. A., and Chabay, I. S., "Particle Doppler Shift Spectrometry: Accurate Size Determination of 5-15 um Aerosol," J. Phys: Chem. 84, 1607-1611 (1980).

Currie, L. A., "Krypton-81" with (H. Oeschger) and "Small Radiocarbon Samples," in Workshop on Dating 01d Groundwater (S. N. Davis, Ed.), University of Arizona, (1978).

Cooper, J. A., Currie, L. A., and Klouda, G. A., "Evaluation of Carbon-14 as a Unique Tracer to Determine the Maximum Impact of Contemporary Carbon Sources of Atmospheric Particulates in the Portland and Eugene Airsheds," Final Report: Oregon Department of Environmental Quality (1979).

Ritter, G. L. and Currie, L. A., "Resolution of Spectral Peaks: Use of Empirical Peak Shape," Proceedings of the Topical Conference on Computers in Activation Analys is of the American Nuclear Society, (B. S. Carpenter, M. D. D'Agostino, and H. P. Yule, Eds.), DOE Symposium Series $\underline{49}, 39$ (1979).

Currie, L. A. and Kropschot, R. H., "Foresight-Volume I. Societal Aspects of Hydrogen Energy Systems," published by the U.S. House of Representatives, Committee on Science and Technology, Part A, p. 1-134 (1979).

Currie, L. A., Noakes, J. E., and Breiter, D. N., "Measurement of Small Radiocarbon Samples: Power of Alternative Methods for Tracing Atmospheric Hydrocarbons, " in Radiocarbon Dating, R. Berger and H. E. Suess, Eds., (University of California Press, 1979), p. 158-175.

Currie, L. A. and Klouda, G. A., "Discrimination of Natural From Anthropogenic Carbonaceous Pollutants Through Mini-Radiocarbon Measurements," NBS Special Publication 572, (1980), p. 68-73. 
Currie, L. A., "Scientific Uncertainty and Societal Decisions: The Challenge to the Analytical Chemist," Analytical Letters, 13, 1-31 (1980).

Elkins, J. W., "Determination of Dissolved Nitrous Oxide in Aquatic Systems by Gas Chromatography Using Electron-Capture Detection and Multiple Phase Equilibration," Anal. Chem., 52, 263-267 (1980).

Etz, E. S. and Blaha, J. J., "Laser-Raman Microprobe Analysis of Particles in Pollution Studies," Trans. Am. Nucl. Soc., 33, 241 (1979).

Abraham, J. L. and Etz, E. S., "Molecular Microanalysis of Pathological Specimens in situ with a Laser-Raman Microprobe," Science 206, 716-718 (1979).

Etz, E. S. and Blaha, J. J., "Scope and Limitations of Single Particle Analysis by Raman Microprobe Spectroscopy," in NBS Special Publication 533, Characterization of Particles (1980), p. 153-197.

Fletcher, R. A., Bright, D. S., and Chabay, I. S., "Low Reynolds Number Fluid Flow Induced by Settling Aerosol and Detected by the Particle Doppler Shift Spectrometer," J. Chem. Phys. 84, 1611-1614 (1980).

Marinenko, R. B., Heinrich, K. F. J., Myklebust, R. L., and Fiori, C. E., "Crystal Efficiency Determination for Relative Line Intensity Measurements," Proceedings of the 15th Annual Conference of the Microbeam Analysis Society, (San Francisco Press, 1980), p. 56-58.

Menis, O. and Garn, P. D., "The State-of-the-Art of Thermal Analysis," NBS Special Publication 580, (1980).

Fiori, C. E., Myklebust, R. L., and Newbury, D. E., "A Catalogue of Artifacts Observed in Energy-Dispersive X-ray Spectrometry and Their Influence on Analysis," in Microbeam Analysis in Biology, (C. Lecheme, Ed.), Academic Press, New York, NY, (1979), p. 225-263.

Fiori, C. E. and Myklebust, R. L., "A Simplex Method for Fitting Gaussian Profiles to X-ray Spectra Obtained with an Energy-Dispersive Detector," Computers in Activation Analysis and Gamma-Ray Spectroscopy, DOE Symposium Series 49, CONF-780421, (1979), p. 139-149.

Myklebust, R. L., Newbury, D. E., and Smal1, J. A., "Backscatter Loss in the X-ray Continuum, "Proceedings of the 15th Annual Conference of the Microbeam Analysis Society, (San Francisco Press, 1980), p. 49-50.

Newbury, D. E., "Instrumental Effects on Quantitative Analysis by Secondary Ion Mass Spectrometry," in Secondary Ion Mass Spectrometry II, (A. Benninghaven, C. A. Evans, Jr., R. A. Powell, R. Shimizu, and H. A. Storms, Eds.), Springer-Verlag, Berlin, (1979), p. 53-57. 
Newbury, D. E. and Myklebust, R. L., "Calculation of Electron Beam Spreading in Composite Thin Foil Targets," Proceedings of the 15 th Annual Conference of the Microbeam Analysis Society, (San Francisco Press, 1980), p. 173-175.

Newbury, D. E., "Secondary Ion Mass Spectrometry for the Analysis of Single Particles," in NBS Special Publication 533, Characterization of Particles (1980), p. 139-152.

Newbury, D. E., Myklebust, R. L., Heinrich, K. F. J., and Fiori, C. E., "Specialist Workshop on Energy Dispersive X-ray Spectrometry," Scanning, 3 , 43-44 (1980).

Fiori, C. E. and Newbury, D. E., "Artifacts in Energy Dispersive X-ray Spectrometry in the Scanning Electron Microscope (II)," Proceedings of the Scanning Electron Microscopy/1980, the Annual Review of Scanning Electron Microscopy, (0. Johari, Ed.), (1980), p. 251-258.

Newbury, D. E., Myklebust, R. L., Heinrich, K. F. J., and Sma11, J. A., "Monte Carlo Electron Trajectory Simulation - an Aid for Particle Analysis," in NBS Special Publication 533, Characterization of Particles (1980), p. 39-62.

Pella, P. A., "The Development of Potential Thin Standards for Calibration of X-ray Fluorescence Spectrometry, NBS/EPA Report No. EPA-600/7-80-031, February 1980.

Gills, T. E. and Rook, H. L., "Specimen Bank Research at the National Bureau of Standards to Insure Proper Scientific Protocols for the Sampling, Storage, and Analysis of Environmental Materials," in Monitoring Environmental Materials and Specimen Banking, (N. -P. Luepke, Ed.), Martinus Nijhoff Publishers, (1979).

Simons, D. S., "Ion Microprobe, Ion Microscope, and Laser Microprobe Mass Analysis of Particulates," Proceedings of the 15th Annual Conference of the Microbeam Analysis Society, (San Francisco Press, 1980), p. 178-180.

Sma11, J. A. and Steel, E. B., "An Overview of NBS-EPA Program for the Development of Standards for Use in the Analysis of Environmental Samples for Asbestos by Electron Microscopy," to be published in the Proceedings of the 3rd Colloquium on Dust Measuring Technique and Strategy, Cannes, France (1980).

Sma11, J. A., Newbury, D. E., and Myklebust, R. L., "Instrumental Effects on the Generation of Continuum from Pure Element Targets," Proceedings of the 15th Annual Conference of the Microbeam Analysis Society, (San Francisco Press, 1980), p. 53-55.

Smal1, J. A., Heinrich, K. F. J., Newbury, D. E., Myklebust, R. L., and Fiori, C. E., "Procedure for the Analysis of Single Particles with the Electron Probe," in NBS Special Publication 533, Characterization of Particles (1980), p. 29-38. 
Steel, E. B., "Optical Microscopy of Particles," in NBS Special Publication 533, Characterization of Particles. (1980), p. 5-11.

Bass, A. M., Glasgow, L. C., Miller, C., Jesson, J. P., and Filkin, D. L., "Temperature Dependent Absorption Cross Sections for Formaldehyde $\left(\mathrm{CH}_{2} \mathrm{O}\right)$ : The Effect of Formaldehyde on Stratospheric Chlorine Chemistry," to be published in Planetary and Space Science.

Blaha, J. J., Etz, E. S., and Heinrich, K. F. J., "Raman Microprobe Analysis of Stationary Source Particulate Pollutants," to be published as an EPA Final Report.

Blaha. J. J., "Raman Microprobe Spectroscopic Analysis," to be published in Vibrational Spectra and Structure, Volume $X$.

Blaha, J. J. and Rosasco, G. J., "Raman Microprobe Characterization of Urea: n-Paraffin Inclusion Compounds," to be published in the J. Raman Spectroscopy.

Dornhaus, R., Benner, R. E., Chang, R. K., and Chabay, I.S., "Surface Plasmon Contribution to SERS," to be published in Surface Science.

Currie, L. A., Ed., Nuclear and Chemical Dating Techniques, to be published as a monograph in the American Chemical Society Symposium Series.

Currie, L. A., Klouda, G. A., and Cooper, J. A., "Mini-Radiocarbon Measurements, Chemical Selectivity, and the Impact of Man on Environmental Pollution and Climate," Proceedings of the 10th International Radiocarbon Conference (in press).

Currie, L. A., "Contemporary Elemental Carbon," to be published in the Proceedings of the Symposium on Particulate Carbon: Atmospheric Life Cycle.

Currie, L. A., "The Origin of Carbonaceous Species in the Atmosphere Deduced by Radiocarbon," ASTM Symposium on Sampling and Analysis of Toxic Organics in the Atmosphere (in press).

Voorhees, K. J., Kunen, S. M., Durfee, S. L., Currie, L. A., and Klouda, G. A., "The Determination of Source Contribution of Organic Matter in Atmospheric Particulates by Pyrolysis/Mass Spectrometry and 14C Analysis," submitted to Analytical Chemistry.

Currie, L. A. and Polach, H. A., "Exploratory Analysis of the International Radiocarbon Cross-Calibration Data: Consensus Values and Interlaboratory Error," Proceedings of the 10th International Radiocarbon Conference (in press).

Currie, L. A. and Klouda, G. A., "Counters, Accelerators, and Chemistry," to be published in Nuclear and Chemical Dating Techniques.

Swanson, J., Fairha11, A. and Currie, L. A., "Carbon Isotope Analysis of Sedimentary Polycyclic Aromatic Hydrocarbons," submitted to Nature. 
Currie, L. A., "The Isotopic Dimension" and "Model, Error Structure and Measurement Accuracy," Proceedings of the Workshop on Receptor Modeling (in press).

Etz, E. S. and Blaha, J. J., "Raman Microprobe Spectra of Nearly Opaque Particles," to be published in Analytical Chemistry.

Hodgeson, J. A. and Fatiadi, A. J., "A Survey on Research Needs on Personal Samples for Toxic Organic Compounds," EPA Report No. EPA/600/4-80-042, (to be published).

Hughes, E. E. and Suddueth, J. E., "A Computer Operated Gas Analys is System (COGAS) for the sequential Analysis of Multiple Samples of Compressed Gases," to be published in Analytical Chemistry.

Myklebust, R. L., Small, J. A., and Newbury, D. E., "Quantitative Electron Probe Microanalysis of Fly Ash Particles," to be published in the Transaction of the American Nuclear Society.

Newbury, D. E. and Greenwald, S., "Observations on the Mechanisms of High Resistance Junction Formation in Aluminum Wire Connections," Journal of Research (in press).

Joy, D. C., Newbury, D. E., and Davidson, D. L., "Electron Channeling Patterns (ECP) in the Scanning Electron Microscope," to be published in Physical Electron Microscopy, Volume 1, Von Nostrand, New York.

Newbury, D. E. and Myklebust, R. L., "Monte Carlo Electron Trajectory Simulation of Signals at Interfaces in Analytical Electron Microscopy," to be published in the Proceedings of the 9th International Conference on X-ray Optics and Microanalysis.

Newbury, D. E., "Microanalysis of Individual Layered Particles by Secondary Ion Mass Spectrometry," to be published as an EPA Final Report.

Newbury, D. E., "The Impact of Instrumental Sensitivity Variations on Quantitative Analysis With the Local Thermal Equilibrium Model in Secondary Ion Mass Spectrometry," to be published in the Proceedings of the 9th International Conference on X-ray Optics and Microanalysis.

Stone, R. E., Walter, F. J., Blackburn, D. H., Pella, P. A., and Kramer, H. W., "A Standard Technique for Measuring Window Absorption and Other Efficiency Losses in Semiconductor Energy Dispersive X-ray Spectrometry," to be publ ished in X-ray Spectrometry.

Kingston, H. and Pella, P. A., "X-ray Spectrometric Analys is of Trace Metals in Environmental and Biological Samples Using Cation Exchange Resin-Loaded Filters," to be published in Analytical Chemistry. 
2. Talks

Bass, Arnold M., "Temperature Dependent Absorption Cross Sections for Formaldehyde $\left(\mathrm{CH}_{2} \mathrm{O}\right)$ : The Effect of Formaldehyde on Stratospheric Chlorine Chemistry," NATO Advanced Study Institute of Atmospheric Ozone, Portugal, October 1979.

Currie, Lloyd A., "Cosmic Carbon," Washington, DC, October 1979.

Chabay, Ilan S., "Surface Plasmon Enhanced Inelastic Light Scattering From Thin Films and Microspheres," New York, NY, November 2, 1979.

Etz, Edgar S., "Raman Microprobe Characterization of Nitrogen-Sulfur Compounds in Stratospheric Aerosols," Moffet Field, CA, November 8, 1979.

Chabay, Ilan S., "Raman Spectroscopy of Molecules Near Surfaces: Bulk Liquids and Adsorbed Monolayers," New York, NY, November 9, 1979.

Etz, Edgar S., "Laser-Raman Microprobe Analysis of Particles in Pollution Studies," San Francisco, CA, November 15, 1979.

Currie, Lloyd A., "Environmental Radiocarbon: Measurement by Low-Level Counting and Accelerator Mass Spectrometry," Washington, DC, November 15, 1979.

Steel, Eric B., "Mineralogical Characteristic of Asbestos," Tuscon, Arizona, November 1979.

Chabay, Ilan S., "Two Flavors of Raman Spectroscopy: Concentration Fluctuations in Turbulent Mixing and CARS in Waveguides," Wilmington, DE, December 17, 1979.

McKenzie, Raymond L., "Measurement of Aerosol Sulfur Using an Electrostatic Precipitator-FPD System," Ann Arbor, MI, December 18, 1979.

McKenzie, Raymond L., "Evaluation of a New Fast-FPD and Its Use With the PEP," St. Louis, MO, December 20, 1979.

Elkins, James E., "Dissolved Nitrous Oxide in Coastal Waters Off Peru: A Nitrification Source and Deritrification Sink," Los Angeles, CA, February 7, 1980.

Smal1, John A., "Particle Analysis at NBS," Washington, DC, February 15, 1980.

Steel, Eric B., "Microscopic Characterization of Fibrous Minerals From Pennsylvania Crushed Stone Quarries," Washington, DC, February 14, 1980.

Blaha, John B., "Microanalysis By Vibrational Spectroscopy With a Raman Microprobe," Athens, GA, February 19, 1980. 
Currie, Lloyd A., "The Isotopic Dimension," Durham, NC, February 26, 1980.

Chabay, Ilan S., "Evanescently Excited Raman Spectra of Liquid Benzene and Adsorbed Monolayers," New York, NYY, February 29, 1980.

Newbury, Dale E., "Recent Progress in the Theory and Application of Microprobe Analys is," Bethlehem, PA, February 29, 1980.

Currie, Lloyd A., "Counters, Accelerators, and Chemistry," Houston, TX, March 25, 1980.

Etz, Edgar S., "Raman Microprobe Identification of Nitrogen-Sulfur Compounds in Stratospheric Aerosols," Houston, TX, March 27, 1980.

Chabay, Ilan S., "Raman Measurements of Concentration Fluctuations in Reactive and Non-Reactive Systems," New York, NY, April 9, 1980.

Newbury, Dale E., "Artifacts in Energy Dispersive X-ray Spectrometry in the Scanning Electron Microscope:" Chicago, IL, April 2, 1980.

Currie, Lloyd A., "Radiocarbon as a Tracer for Toxic Organics," Denver, C0, Apri1 2, 1980.

Chabay, Ilan S., "Time, Frequency, and Probability Analys is of Raman Signals From Concentration Fluctuations of Individual Species in Turbulent Mixing Flow," Livermore, CA, May 2, 1980.

Kagann, Robert H., "The Determination of Absolute Infrared Absorption Line Strengths of Nitrous Oxide With a Diode Laser Spectrometer," Washington, DC, May 14, 1980.

Chabay, Ilan S., "Time, Frequency, and Probability Analys is of Raman Signals From Concentration Fluctuations of Individual Species in Turbulent Mixing Flow," New York, NY, May 15, 1980.

Simons, David S., "Ion Microscope and Ion Microprobe Analys is of Particulates," New York, NY, May 27, 1980.

Small, John A., "An Overview of NBS-EPA Program for Development of Standards for Use in the Analys is of Environmental Samples for Asbestos by Electron Microscopy," Cannes, France, June 11, 1980.

Kagann, Robert H., "Diode Laser Intensity and Line Width Measurements in the $\mu \mathrm{l}$ and Adjacent Absorption Bands of $\mathrm{N}_{2} \mathrm{O}$, "Columbus, $\mathrm{OH}$, June $19,1980$.

Etz, Edgar S., "A New Raman Microprobe with Multichannel Optical Detector--Characteristics and Applications," Postdam, NY, July $1,1980$. 
Currie, Lloyd A., "On the Question of Detectability," Washington, DC, Ju1y 8, 1980.

Rook, Harry L., "The Concept of Reality of Traceability in Gas Standards," Johnson, VT, July 16, 1980.

Hughes, Ernest E., "Preparation and Certification of Gaseous Standard Reference Materials," Johnson, VT, July 15, 1980.

Etz, Edgar S., "Materials Characterization by Raman Microprobe Spectroscopy," Albuquerque, NM, July 17, 1980.

Etz, Edgar S., "The Development of Instrumentation for Microanalysis by Raman Microprobe Spectroscopy," Livermore, CA, July 24, 1980.

Bass, Arnold M., "New Measurements of Ozone Cross-Sections," Boulder, C0, August 4, 1980.

Myklebust, Robert L. "Back-Scatter Loss in the X-ray Continuum," Reno, NV, August 5, 1980.

Pella, Peter A., "X-ray Spectrometric Analysis of Trace Metals in Environmental and Biological Samples Using Exchange Resin-Loaded Filters," Reno, NV, August 1980.

Marinenko, Ryna B., "Crystal Efficiency Determination for Relative Line Intensity Measurements," Reno, NV, August 5, 1980.

Steel, Eric B., "Sputtered Glass Thin Films for Analytical Electron Microscope Standards," Reno, NV, August 6, 1980.

Simons, David S., "Ion Microprobe, Ion Microscope, and Laser Microprobe Mass Analysis of Particulates," Reno, NV, August 6, 1980.

Newbury, Dale E., "Calculation of Electron Bea, Spreading in Composite Thin Foil Targets, Reno, NV, August 6, 1980.

Blaha, John J., "Some Experimental Problems in the Raman Analysis of Microsamples," Reno, NV, August 6, 1980.

Currie, Lloyd A., "Source Identification of Atmospheric Particulate Matter By Py-MS/Pattern Recognition Procedures," Las Vegas, NV, August 16, 1980.

Etz, Edgar S., "Raman Microprobe Spectroscopy with Applications to Biomedical Studies," Bethesda, MD, August 18, 1980.

Newbury, Dale E., "Signal Processing and Video Display in Scanning Electron Microscope," Brighton, England, August 18, 1980 
Bright, David S., "A New Personal Sampler for Ambient Inhalable Particles," Boston, MA, August 19, 1980.

Chabay, Ilan S., "Raman Spectral Probe of Evanescent Field Components At Metal and Dielectric Interfaces," Wolfeboro, NH, August 20, 1980.

Newbury, Dale E., "Methods of Quantitative Analys is in Secondary Ion Mass Spectrometry," Brighton, England, August 21, 1980.

Newbury, Dale E., "Monte Carlo Electron Trajectory Simulation of X-ray Signals at Interfaces," The Hague, The Netherlands, Agust 26, 1980.

Newbury, Dale E., "The Impact of Instrumental Sensitivity Variations on Analysis With the Local Thermal Equilibrium Model in Secondary Ion Mass Spectrometry," The Hague, The Netherlands, August 28, 1980.

\section{Committee Assignments}

Harry Rook

Member, ASTM D-22.05 Calibration

ASTM D-22.07 Precision and Accuracy of Methods

Office of Water Data Coordination, Committee $5 \mathrm{~F}$

Ernest Hughes

Member, IS0/TC 158 Gas Analysis

ISO/TC 158/SC- 7 Methods for Preparation and Definition of

Gas Mixtures for Calibration

IS0/TC 158/WG-2 Transfer Lines and Sampling

ISO/TC 158/WG-3 Evaluation of the Characteristics of Analys is

IS0/TC 158/WG-4 Analysis of Natural Gas

Secretary, ISO/TC 158/WG-1 Terminology

Arnold Bass

Member, ASTM D-22.02 Methods of Sampling and Analysis

ASTM D-22.03 Monitoring Instrumentation

ASTM D-22.05 Calibration

ASTM D-22.07 Precision and Accuracy of Methods

David Bright

Member, ASTM E-29.04 Liquid Particle Measurements

Il an Chabay

Member, NBS Postdoctoral Selection Committee

Lloyd Currie

Member, Intersociety Committee - Methods of Air Sampling and Analys is

Analytical Letters - Editorial Board

International Committee for the Calibration of the

Radiocarbon Time Scale

Model Validation Subcommittee - Receptor Modeling Workshop 
William Dorko

Member, American Chemical Society, Washington Section, Board of Managers

Edgar Etz

Member, ASTM D-22 Methods of Sampling and Analysis of Atmospheres

Jimmie Hodgeson

Member, ASTM D-22 Atmospheric Analysis

ASTM D-22.02 Manual Methods

ASTM D-22.03 Instrumental Methods

ISO/TC 146/SC3 Air Quality/Ambient Atmospheres

Raymond McKenzie

Member, ASTM D-22 Sampling and Atmospheric Analys is

ASTM D-22.03 Monitoring Instrumentation

ASTM E-29 Particle Size Measurement

ASTM E-29.04 Characterization of Liquid Particles

Robert Myklebust

Member, ASTM E-2 Emission Spectroscopy

ASTM E-2.04 Standard Reference Materials

Dale Newbury

Chairman, Microbeam Analys is Society

Technical Program Committee

National Council for the MAS

Member, ASTM E-42 Surface Analysis Journal of Microscopy, Editorial Review Board Scanning, Editorial Review Board

Peter Pella

Member, ASTM E-2 Emission Spectroscopy

ASTM E-2.02 Statistics, Calibration and Standardization

Gerald Sleater

Member, ASTM D-22 Sampling and Analys is of Atmospheres

ASTM D-22.06 Source Sampling

ASTM D-22.07 Precision and Accuracy of Methods

4. other

a. Seminars

November 19, 1979 Dr. Paul D. Garn

University of Akron

Akron, Chio

Problem Solving Via Thermoanalytical Techniques 
November 23, 1979 Dr. Sydney Leach

University of Paris

Paris, France

Radiationless Transitions in Molecular Ions

December 3, 1979 Dr. Bruce Kowalski

University of Washington

Seattle, Washington

Chemometrics in Analytical Chemistry

January 29, 1980 Dr. Otto Grubner

Harvard Iniversity

Boston, Massachusetts

Improvement in Gas Chromatographic Separation by

Specific Activation Compounds

March 17, 1980

Ernest Schumacher

University of Bern

Bern, Switzerland

Spectra and Electronic States of Metal Clusters and their Ions

March 26, 1979

Dr. Ray F. Weiss

Scripps Institute of Oceanography

LaJolla, California

The Spatial and Temporal Distribution of Tropospheric Nitrous oxide

Apri1 15, 1980

Dr. Ken Purser

General Ionex, Inc.

Newbury Port, Massachusetts

U1 trahigh Sensitivity Mass Spectrometry

May 7,1980

Walter Anthony Patrick Nicholson

University of Glasgow

Glasgow, Scotland

Determination of Elemental Concentrations in Low

Atomic Number Matrices by X-ray Microanalysis

June 4,1980

Joseph P. Krasnec

National Center for Atmospheric Research

Boulder, Colorado

Electron Capture Detection Gas Chromatography

July 14, $1980 \quad$ Dr. Yuk Ling Yung

Cal Tech.

Pasadena, California

Photochemistry of Heavy Hydrocarbons in the

Atmosphere 
August 4, 1980

Paul E. Wagner Universität Wien

Vienna, Austria

Nucleation and Droplet Growth in Supersaturated

Vapors

August 6,1980 Jeff Klein

University of Pennsylvania

Philadelphia, Pennsylvania

Sample Preparation for Accelerator Mass Spectrometry, and Fitting the Radiocarbon Calibration Curve

August 28, 1980 Pam Bumsted

Anthropology Department

University of Massachuetts

Amherst, Massachusetts

Dr. Thomas Hoering

Geophysical Laboratory

Carnegie Institute

Pittsburgh, Pennsylvania

Use of the Natural Stable Isotopes of Carbon, Hydrogen, Oxygen and Nitrogen and Trace Elements to Reconstruct Past Environments and Diets

b. Workshops

"Measurement of Atmospheric Fluorocarbons and Nitrous Oxide" National Bureau of Standards - November 16-17, 1979.

"Symposium on Nuclear and Chemical Dating Techniques" Houston, Texas - March 23-28, 1980.

\section{c. Standard Reference Materials}

A large number of analyses were performed leading to the certification of 64 SRM's. These are separated by group and are 1isted below:

Gas Metrology Group

$1625 \mathrm{SO}_{2}$ Permeation Tube, $10 \mathrm{~cm}$
$1626 \mathrm{SO}_{2}$ Permeation Tube, $5 \mathrm{~cm}$
$1627 \mathrm{SO}_{2}$ Permeation Tube, $2 \mathrm{~cm}$
$1658 \mathrm{CH}_{4} /$ Air, $1 \mathrm{ppm}$
$1659 \mathrm{CH}_{4} / \mathrm{Air}, 10 \mathrm{ppm}$
$1660 \mathrm{CH}_{4}$ and $\mathrm{C}_{3} \mathrm{H}_{8} / \mathrm{Air}, 4$ and $1 \mathrm{ppm}$


$1662 \mathrm{SO}_{2} / \mathrm{N}_{2}, 1000 \mathrm{ppm}$

$1663 \mathrm{SO}_{2} / \mathrm{N}_{2}, 1500 \mathrm{ppm}$

$1665 \mathrm{C}_{3} \mathrm{H}_{8} / \mathrm{Air}, \quad 3 \mathrm{ppm}$

$1666 \mathrm{C}_{3} \mathrm{H}_{8} / \mathrm{Air}, \quad 10 \mathrm{ppm}$

$1667 \mathrm{C}_{3} \mathrm{H}_{8} /$ Air, $\quad 50 \mathrm{ppm}$

$1668 \mathrm{C}_{3} \mathrm{H}_{8} / \mathrm{Air}, 100 \mathrm{ppm}$

$1669 \mathrm{C}_{3} \mathrm{H}_{8} / \mathrm{Air}, 500 \mathrm{ppm}$

$1674 \mathrm{CO}_{2} / \mathrm{N}_{2}, \quad 7 \%$

$1675 \mathrm{CO}_{2} / \mathrm{N}_{2}, 14 \%$

$1677 \mathrm{CO} / \mathrm{N}_{2}, \quad 10 \mathrm{ppm}$

$1678 \mathrm{CO} / \mathrm{N}_{2}, \quad 50 \mathrm{ppm}$

$1679 \mathrm{CO} / \mathrm{N}_{2}, \quad 100 \mathrm{ppm}$

$1680 \mathrm{CO} / \mathrm{N}_{2}, \quad 500 \mathrm{ppm}$

$1681 \mathrm{CO} / \mathrm{N}_{2}, 1000 \mathrm{ppm}$

$1683 \mathrm{NO} / \mathrm{N}_{2}, \quad 50 \mathrm{ppm}$

$1684 \mathrm{NO} / \mathrm{N}_{2}, \quad 100 \mathrm{ppm}$

$1685 \mathrm{NO} / \mathrm{N}_{2}, \quad 250 \mathrm{ppm}$

$1686 \mathrm{NO} / \mathrm{N}_{2}, \quad 500 \mathrm{ppm}$

$1687 \mathrm{NO} / \mathrm{N}_{2}, 1000 \mathrm{ppm}$

2612 CO/Air, 10 ppm

2613 C0/Air, 18 ppm

2614 C0/Air, 43 ppm

$2627 \mathrm{NO} / \mathrm{N}_{2}, \quad 5 \mathrm{ppm}$

$2628 \mathrm{NO} / \mathrm{N}_{2}, \quad 10 \mathrm{ppm}$

$2629 \mathrm{NO} / \mathrm{N}_{2}, \quad 20 \mathrm{ppm}$

$2630 \mathrm{NO} / \mathrm{N}_{2}, 1500 \mathrm{ppm}$

$2631 \mathrm{NO} / \mathrm{N}_{2}, 3000 \mathrm{ppm}$

$2643 \mathrm{C}_{3} \mathrm{H}_{8} / \mathrm{N}_{2}, \quad 100 \mathrm{ppm}$

$2644 \mathrm{C}_{3} \mathrm{H}_{8} / \mathrm{N}_{2}, \quad 250 \mathrm{ppm}$

$2645 \mathrm{C}_{3} \mathrm{H}_{8} / \mathrm{N}_{2}, \quad 500 \mathrm{ppm}$

$2646 \mathrm{C}_{3} \mathrm{H}_{8} / \mathrm{N}_{2}, 1000 \mathrm{ppm}$

$2647 \mathrm{C}_{3} \mathrm{H}_{8} / \mathrm{N}_{2}, 2500 \mathrm{ppm}$

$2648 \mathrm{C}_{3} \mathrm{H}_{8} / \mathrm{N}_{2}, 5000 \mathrm{ppm}$

$2649 \mathrm{C}_{3} \mathrm{H}_{8} / \mathrm{N}_{2}, \quad 1 \%$

$2650 \mathrm{C}_{3} \mathrm{H}_{8} / \mathrm{N}_{2}, \quad 2 \%$

$2651 \mathrm{C}_{3} \mathrm{H}_{8} / \mathrm{N}_{2}+5 \% \mathrm{O}_{2}, 100 \mathrm{ppm}$

$2652 \mathrm{C}_{3} \mathrm{H}_{8} / \mathrm{N}_{2}+10 \% \mathrm{O}_{2}, 100 \mathrm{ppm}$

$2653 \mathrm{NO}_{2} /$ Air, $250 \mathrm{ppm}$

$2654 \mathrm{NO}_{2}$ /Air, $500 \mathrm{ppm}$

$2655 \mathrm{NO}_{2}$ /Air, $1000 \mathrm{ppm}$

$2656 \mathrm{NO}_{2} / \mathrm{Air}, 2500 \mathrm{ppm}$ 


$$
\begin{array}{lll}
2657 & \mathrm{O}_{2} / \mathrm{N}_{2}, & 2 \% \\
2658 & \mathrm{O}_{2} / \mathrm{N}_{2}, & 10 \% \\
2659 & \mathrm{O}_{2} / \mathrm{N}_{2}, & 21 \% \\
2682 & \mathrm{SO}_{2} / \mathrm{N}_{2}, & 50 \mathrm{ppm} \\
2683 & \mathrm{SO}_{2} / \mathrm{N}_{2}, & 100 \mathrm{ppm}
\end{array}
$$

W. D. Dorko, E. E. Hughes, R. Myers, W. Thorn, R. Miller, J. Mackey,

G. Rhoderick, G. Sleater, W. P. Schmidt, J. Suddueth

\section{Microanalysis Research Group}

479a, Fe-Cr-Ni Alloy for Microanalys is

470, Mineral Glasses for Microanalysis (K-411 and K-412)

30, Glasses for Microanalysis

R. B. Marinenko, R. L. Myklebust

\section{X-Ray Fluorescence Group}

1622 a Sulfur in residual fuel oil

C1173 Steel, low alloy

C1173a Steel, low alloy

477 Glass disks, energy loss measurements, EDXRF

1262a Steel, low al loy, AISI94B17(Mod), renewal

1263a Steel, low alloy, $\mathrm{Cr}-\mathrm{V}$ (Mod), renewal

1264a Steel, low alloy, high carbon (Mod), renewa 1

1261 a Steel, low alloy, AISI4340, renewal

P. A. Pella, J. R. Sieber 


\begin{tabular}{|c|c|c|}
\hline $\begin{array}{l}\text { U.S. DEPT. OF COMM. } \\
\text { BIBLIOGRAPHIC DATA } \\
\text { SHEET }\end{array}$ & $\begin{array}{l}\text { 1. PUBLICATION OR REPORT NO. } \\
\text { NBSIR } 80-2164\end{array}$ & 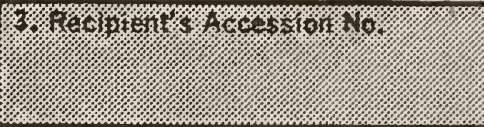 \\
\hline \multirow{2}{*}{\multicolumn{2}{|c|}{$\begin{array}{l}\text { 4. TITLE AND SUBTITLE } \\
\text { Annual Report 1980, Center for Analytical Chemistry }\end{array}$}} & $\begin{array}{l}\text { 5. Publication Date } \\
\text { December } 1980\end{array}$ \\
\hline & & 6. Perfoming Organization Code \\
\hline \multicolumn{2}{|c|}{$\begin{array}{l}\text { 7. AUTHOR(S) } \\
\text { C. W. Reimann, R. A. Velapoldi, L. B. Hagan, J. K. Taylor }\end{array}$} & 8. Performing Organ. Report No. \\
\hline \multicolumn{2}{|c|}{ 9. PERFORMING ORGANIZATION NAME AND ADDRESS } & 10. Project/Task/Work Unit No. \\
\hline \multicolumn{2}{|c|}{$\begin{array}{l}\text { NATIONAL BUREAU OF STANDARDS } \\
\text { DEPARTMENT OF COMMERCE } \\
\text { WASHINGTON, DC } 20234\end{array}$} & 11. Contract/Grant No. \\
\hline \multirow{2}{*}{\multicolumn{2}{|c|}{ 12. SPONSORING ORGANIZATION NAME AND COMPLETE ADDRESS (Street, City, State, ZIP) }} & 13. Type of Report \& Period Covered \\
\hline & & 274. Sporsoting Agercy Code \\
\hline
\end{tabular}

15. SUPPLEMENTARY NOTES

Document describes a computer program; SF-185, FIPS Software Summary, is attached.

16. ABSTRACT (A 200-word or less factual summary of most significant information. If documerit includes a significant bibliography or literature survey, mention it hero.)

This report summarizes the technical activities of the Center for Analytical Chemistry at the National Bureau of Standards. It emphasizes activities over the Fiscal Year, 1980 in the Instrumentation Development Group, the Inorganic Analytical Research Division, the Organic Analytical Research Division, and the Gas and Particulate Science Division. In addition, it describes certain special activities in the Center including quality assurance and voluntary standardization coordination, the National Environmental Specimen Bank, and service analysis coordination.

17. KEY WORDS (six to twelve entries; alphabetical order; capitalize only the first letter of the first key word unless a proper name; separated by semicolons)

Analytical chemistry; analytical services; gas and particulate science; inorganic analytical chemistry; National Environmental Specimen Bank; organic analytical chemistry; quality assurance; voluntary standardization.

\begin{tabular}{|c|c|c|}
\hline 18. AVAILABILITY $\square$ Unlimited & $\begin{array}{l}\text { 19. SECURITY CLASS } \\
\text { (THIS REPORT) }\end{array}$ & $\begin{array}{l}\text { 21. NO. OF } \\
\text { PRINTED PAGES }\end{array}$ \\
\hline X For Official Distribution. Do Not Release to NTIS & UNCLASSIFIED & \\
\hline $\begin{array}{l}\square \text { Order From Sup. of Doc., U.S. Government Printing Office, Wasinington, DC } \\
20402 \text {, SD Stock No. SNO03-003- }\end{array}$ & $\begin{array}{l}\text { 20. SECURITY CLASS } \\
\text { (THIS PAGE) }\end{array}$ & 22. Price \\
\hline $\begin{array}{l}\square \text { Order From National Technical Information Service (NTIS), Springfield, } \\
\text { VA. } 22161\end{array}$ & UNCLASSIFIED & \\
\hline
\end{tabular}


$181 \%$ 
